Supporting Information

$$
\text { for }
$$

\title{
Copper-Catalyzed $\beta$-Lactam Formation Initiated by 1,3-Azaprotio Transfer of Oximes and Methyl Propiolate
}

\author{
Zhenjie Qi and Shaozhong Wang*
}

State Key Laboratory of Coordination Chemistry, Jiangsu Key Laboratory of Advanced Organic Materials, School of Chemistry and Chemical Engineering, Nanjing University, 163 Xianlin avenue, Nanjing 210023, China.

E-mail: wangsz@nju.edu.cn 


\section{Table of Contents}

1. General informations SI-3

2. General procedure for the copper-catalyzed formation of $\beta$-lactams SI-3

3. Characterization data of $\beta$-lactams 2a-2aq SI-4

4. Control experiment using copper acetylide SI-17

5. Gram scale reaction

6. Procedures and data for compounds 3-7 SI-18

7. X-Ray crystallographic data of compounds $\mathbf{2 g}$ and $\mathbf{2 a b} \quad$ SI-21

8. References SI-23

9. Copies of NMR spectra SI-25 


\section{Genernal informations}

${ }^{1} \mathrm{H}$ NMR were recorded at $400 \mathrm{MHz},{ }^{13} \mathrm{C}$ NMR spectra were recorded at $100 \mathrm{MHz}$. Spectra were recorded in a $\mathrm{CDCl}_{3}$, DMSO- $d_{6}$ solution using the residual protonated solvent as the internal standard, $J$ values are given in hertz. IR spectra were recorded on Fourier Transform infrared spectrometer and listed in $\mathrm{cm}^{-1}$. High-resolution mass spectral analyses (HRMS) were performed on a Q-TOF-MS spectrometer. The dr values were determined by integration of peaks shown in ${ }^{1}$ H NMR spectra. Flash column chromatography was performed over silica gel (300-400 mesh). All commercially available reagents were used without further purification. Oximes were prepared according to the literature procedures. 1-11. Methyl propiolate was purchased from Aladdin Company.

\section{General procedure for the copper-catalyzed formation of $\beta$-lactams}

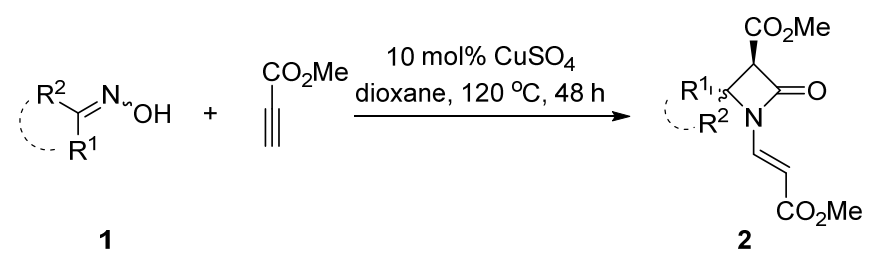

A mixture containing oxime 1 ( $0.3 \mathrm{mmol}, 1.0$ equiv), methyl propiolate $\left(0.9 \mathrm{mmol}, 3.0\right.$ equiv), anhydrous $\mathrm{CuSO}_{4}$ $\left(0.03 \mathrm{mmol}, 0.1\right.$ equiv) and dioxane $(3 \mathrm{~mL})$ was stirred in a sealed tube at $120^{\circ} \mathrm{C}$ for $48 \mathrm{~h}$ on a heating mantle. After cooling, saturated aqueous $\mathrm{NH}_{4} \mathrm{Cl}$ solution $(5 \mathrm{~mL})$ was added and the resulting mixture was diluted with ethyl acetate $(5 \mathrm{~mL})$. The organic layer was separated and the aqueous layer was extracted with EtOAc $(5 \mathrm{~mL} \times 3)$. The combined extracts were washed with brine $(10 \mathrm{~mL})$, dried over anhydrous $\mathrm{Na}_{2} \mathrm{SO}_{4}$. After filtration, the filtrate was concentrated under vacuum and the obtained residue was purified by flash column chromatography on silica gel (petroleum ether (PE)/EtOAc as eluent, the ratio varies from 10:1 to 4:1) to afford $\beta$-lactam 2.

\section{Characterization data of $\beta$-lactams 2a-2ap}

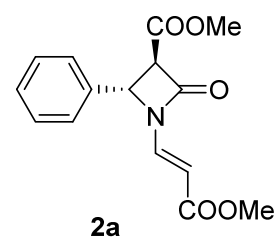

( \pm ) Methyl (3R,4S)-1-((E)-3-methoxy-3-oxoprop-1-en-1-yl)-2-oxo-4-phenylazetidine-3-carboxylate

According to the general procedure, $\beta$-lactam 2a was obtained from benzaldehyde oxime $(36.3 \mathrm{mg}, 0.3 \mathrm{mmol})$ and methyl propiolate $(76.5 \mathrm{mg}, 0.9 \mathrm{mmol})$ as light yellow oil $(65 \mathrm{mg}, 75 \%$ yield, $91 / 9 \mathrm{dr}$; eluent: $\mathrm{PE} / \mathrm{EtOAc}=10: 1$ to 4:1). ${ }^{1} \mathrm{H}$ NMR (400 MHz, $\left.\mathrm{CDCl}_{3}\right) \delta: 7.62-7.59(\mathrm{~d}, J=14.3 \mathrm{~Hz}, 1 \mathrm{H}), 7.36-7.32(\mathrm{~m}, 3 \mathrm{H}), 7.26-7.24(\mathrm{~m}, 2 \mathrm{H}), 5.12(\mathrm{~d}, J=$ $3.1 \mathrm{~Hz}, 1 \mathrm{H}), 5.09-5.05(\mathrm{~d}, J=14.3 \mathrm{~Hz}, 1 \mathrm{H}), 3.97(\mathrm{~d}, J=3.1 \mathrm{~Hz}, 1 \mathrm{H}), 3.76(\mathrm{~s}, 3 \mathrm{H}), 3.60$ (s, $3 \mathrm{H}) \mathrm{ppm} ;{ }^{13} \mathrm{C}$ NMR $(100$ $\left.\mathrm{MHz}, \mathrm{CDCl}_{3}\right) \delta: 166.8,165.8,159.5,134.38,133.6,129.5,129.4,126.1,103.0,64.3,58.6,53.1,51.5$ ppm; IR (Neat): 2953, 1780, 1734, 1630, 1438, 1289, 1161, 960, 838, 729, 697, $497 \mathrm{~cm}^{-1}$; HRMS (ESI) $\mathrm{m} / z:[\mathrm{M}+\mathrm{H}]^{+}$calcd. for 


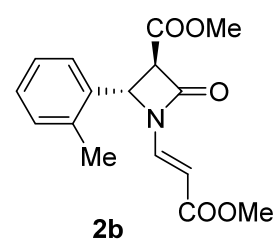

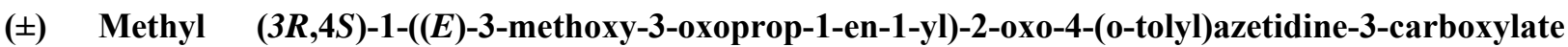

(2b).

According to the general procedure, $\beta$-lactam $2 \mathbf{b}$ was obtained from 2-methylbenzaldehyde oxime (40.5 mg, 0.3 $\mathrm{mmol}$ ) and methyl propiolate $(76.5 \mathrm{mg}, 0.9 \mathrm{mmol})$ as light yellow oil $(66.4 \mathrm{mg}, 73 \%$ yield, $91 / 9 \mathrm{dr}$; eluent: PE/EtOAc $=10: 1$ to $4: 1) .{ }^{1} \mathrm{H}$ NMR $\left(400 \mathrm{MHz}, \mathrm{CDCl}_{3}\right) \delta: 7.69-7.65(\mathrm{~d}, J=14.2 \mathrm{~Hz}, 1 \mathrm{H}), 7.18-7.16(\mathrm{~m}, 3 \mathrm{H}), 7.08-7.05(\mathrm{~m}, 1 \mathrm{H})$, 5.37-5.36 (d, $J=3.2 \mathrm{~Hz}, 1 \mathrm{H}), 5.07-5.04(\mathrm{~d}, J=14.2 \mathrm{~Hz}, 1 \mathrm{H}), 3.86-3.85(\mathrm{~d}, J=3.2 \mathrm{~Hz}, 1 \mathrm{H}), 3.77(\mathrm{~s}, 3 \mathrm{H}), 3.61(\mathrm{~s}, 3 \mathrm{H})$, 2.29 (s, 3H) ppm; ${ }^{13} \mathrm{C}$ NMR $\left(100 \mathrm{MHz}, \mathrm{CDCl}_{3}\right) \delta: 166.7,166.0,159.5,135.5,133.8,132.3,131.1,128.9,126.8,124.3$, 103.0, 63.7, 55.8, 53.1, 51.5, 19.0 ppm; IR (Neat): 2953, 1781, 1709, 1629, 1437, 1289, 1158, 961, 838, 753, $589 \mathrm{~cm}^{-1}$; HRMS (ESI) $m / z:[\mathrm{M}+\mathrm{H}]^{+}$calcd. for $\mathrm{C}_{16} \mathrm{H}_{18} \mathrm{NO}_{5}$ : 304.1179; Found: 304.1172.

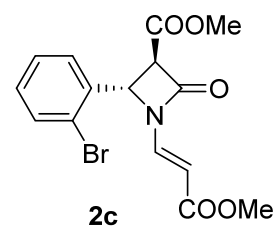

( \pm ) Methyl (2S,3R)-2-(2-bromophenyl)-1-((E)-3-methoxy-3-oxoprop-1-en-1-yl)-4-oxoazetidine-3-carboxylate (2c). According to the general procedure, $\beta$-lactam $2 \mathbf{c}$ was obtained from 2-bromobenzaldehyde oxime (59.4 $\mathrm{mg}, 0.3 \mathrm{mmol}$ ) and methyl propiolate $(76.5 \mathrm{mg}, 0.9 \mathrm{mmol})$ as light yellow oil $(89.2 \mathrm{mg}, 81 \%$ yield, $91 / 9 \mathrm{dr}$; eluent: $\mathrm{PE} / \mathrm{EtOAc}=10: 1$ to 4:1). ${ }^{1} \mathrm{H}$ NMR $\left(400 \mathrm{MHz}, \mathrm{CDCl}_{3}\right) \delta$ : 7.71-7.68 (d, $\left.J=14.3 \mathrm{~Hz}, 1 \mathrm{H}\right), 7.58-7.56(\mathrm{~m}, 1 \mathrm{H}), 7.32-7.28(\mathrm{~m}, 1 \mathrm{H})$, 7.22$7.17(\mathrm{~m}, 1 \mathrm{H}), 7.15-7.12(\mathrm{~m}, 1 \mathrm{H}), 5.49(\mathrm{~d}, J=3.1 \mathrm{~Hz}, 1 \mathrm{H}), 5.13-5.10(\mathrm{~d}, J=14.3 \mathrm{~Hz}, 1 \mathrm{H}), 3.87(\mathrm{~d}, J=3.1 \mathrm{~Hz}, 1 \mathrm{H})$, $3.78(\mathrm{~s}, 3 \mathrm{H}), 3.63(\mathrm{~s}, 3 \mathrm{H}) \mathrm{ppm} ;{ }^{13} \mathrm{C} \mathrm{NMR}\left(100 \mathrm{MHz}, \mathrm{CDCl}_{3}\right) \delta: 166.7,165.7,159.3,133.8,133.7,133.6,130.6,128.2$, 126.6, 122.5, 103.3, 63.5, 58.3, 53.2, 51.6 ppm; IR (Neat): 2952, 2852, 1783, 1709, 1631, 1471, 1289, 1161, 960, 839, 753, $450 \mathrm{~cm}^{-1}$; HRMS (ESI) m/z: [M+H] $]^{+}$calcd. for $\mathrm{C}_{15} \mathrm{H}_{15} \mathrm{BrNO}_{5}$ : 368.0128; Found: 368.0132.

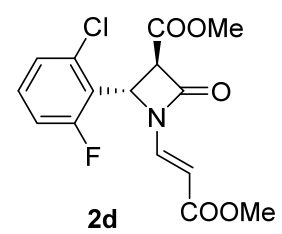

( \pm Methyl (2S,3R)-2-(2-chloro-6-fluorophenyl)-1-((E)-3-methoxy-3-oxoprop-1-en-1-yl)-4-oxoazetidine-3carboxylate (2d). According to the general procedure, $\beta$-lactam 2d was obtained from 2-chloro-6-fluorobenzaldehyde oxime (51.9 mg, $0.3 \mathrm{mmol})$ and methyl propiolate $(76.5 \mathrm{mg}, 0.9 \mathrm{mmol}$ ) as light yellow oil $(85.8 \mathrm{mg}, 84 \%$ yield, $99 / 1$ dr; eluent: $\mathrm{PE} / \mathrm{EtOAc}=8: 1$ to $4: 1) .{ }^{1} \mathrm{H}$ NMR $\left(400 \mathrm{MHz}, \mathrm{CDCl}_{3}\right) \delta: 7.63-7.60(\mathrm{~d}, J=14.2 \mathrm{~Hz}, 1 \mathrm{H}), 7.39-7.34(\mathrm{~m}, 1 \mathrm{H})$, 7.31-7.29 (d, $J=7.5 \mathrm{~Hz}, 1 \mathrm{H}), 7.11-7.06(\mathrm{~m}, 1 \mathrm{H}), 5.84-5.83(\mathrm{dd}, J=3.1,1.0 \mathrm{~Hz}, 1 \mathrm{H}), 5.10-5.07(\mathrm{~d}, J=14.2 \mathrm{~Hz}, 1 \mathrm{H})$, 4.49-4.48 (d, $J=3.1 \mathrm{~Hz}, 1 \mathrm{H}), 3.84(\mathrm{~s}, 3 \mathrm{H}), 3.67$ (s, 3H) ppm; ${ }^{13} \mathrm{C} \mathrm{NMR}\left(100 \mathrm{MHz}, \mathrm{CDCl}_{3}\right) \delta: 166.7,165.7,163.3-$ $160.8(\mathrm{~d}, J=251.4 \mathrm{~Hz}, 1 \mathrm{C}), 159.0,135.2(\mathrm{~d}, J=5.1 \mathrm{~Hz}, 1 \mathrm{C}), 133.5,131.7-131.6(\mathrm{~d}, J=10.3 \mathrm{~Hz}, 1 \mathrm{C}), 126.6(\mathrm{~d}, J=$ $34.3 \mathrm{~Hz}, 1 \mathrm{C}), 119.1-118.9$ (d, $J=12.8 \mathrm{~Hz}, 1 \mathrm{C}), 115.5-115.3$ (d, $J=22.3 \mathrm{~Hz}, 1 \mathrm{C}), 101.6,60.9$ (d, $J=3.6 \mathrm{~Hz}, 1 \mathrm{C}), 53.2$, 52.2, 51.5 ppm; IR (Neat): 2953, 1786, 1709, 1629, 1455, 1246, 1161, 960, 891, 837, 727, 648, $582 \mathrm{~cm}^{-1}$; HRMS (ESI) $m / z:[\mathrm{M}+\mathrm{Na}]^{+}$calcd. for $\mathrm{C}_{15} \mathrm{H}_{13} \mathrm{ClFNNaO}_{5}: 364.0358$; Found: 364.0361 . 


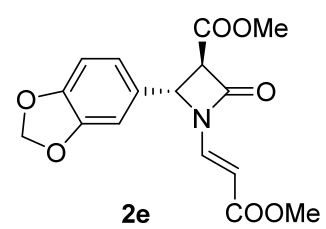

$( \pm) \quad$ Methyl (2S,3R)-2-(benzo[d][1,3]dioxol-5-yl)-1-((E)-3-methoxy-3-oxoprop-1-en-1-yl)-4-oxoazetidine-3carboxylate (2e). According to the general procedure, $\beta$-lactam $2 \mathbf{e}$ was obtained from benzo $[d][1,3]$ dioxole-5carbaldehyde oxime (49.5 mg, $0.3 \mathrm{mmol})$ and methyl propiolate $(76.5 \mathrm{mg}, 0.9 \mathrm{mmol})$ as light yellow oil $(72.6 \mathrm{mg}$, $73 \%$ yield, 93/7 dr; eluent: PE/EtOAc $=4: 1$ to $2: 1) .{ }^{1} \mathrm{H}$ NMR $\left(400 \mathrm{MHz}, \mathrm{CDCl}_{3}\right) \delta: 7.59-7.55(\mathrm{dd}, J=14.3,2.1 \mathrm{~Hz}$, $1 \mathrm{H}), 6.75(\mathrm{~d}, J=1.1 \mathrm{~Hz}, 2 \mathrm{H}), 6.68(\mathrm{~s}, 1 \mathrm{H}), 5.93-5.92(\mathrm{~m}, 2 \mathrm{H}), 5.11-5.07(\mathrm{~m}, 1 \mathrm{H}), 5.03-5.02(\mathrm{~d}, J=2.9 \mathrm{~Hz}, 1 \mathrm{H}), 3.94-$ $3.93(\mathrm{~d}, J=3.1 \mathrm{~Hz}, 1 \mathrm{H}), 3.75(\mathrm{~d}, J=1.8 \mathrm{~Hz}, 3 \mathrm{H}), 3.61(\mathrm{~d}, J=2.0 \mathrm{~Hz}, 3 \mathrm{H}) \mathrm{ppm} ;{ }^{13} \mathrm{C}$ NMR $\left(100 \mathrm{MHz}, \mathrm{CDCl}_{3}\right) \delta: 166.8$, 165.8, 159.5, 148.7, 148.6, 133.5, 128.0, 120.4, 108.9, 105.8, 102.9, 101.6, 64.3, 58.5, 53.1, 51.5 ppm; IR (Neat): 2953, $2905,1780,1707,1629,1446,1244,1163,1035,930,840,730,647,558 \mathrm{~cm}^{-1}$; HRMS (ESI) $m / z:[\mathrm{M}+\mathrm{H}]^{+}$calcd. for $\mathrm{C}_{16} \mathrm{H}_{16} \mathrm{NO}_{7}$ : 334.0921; Found: 334.0925.

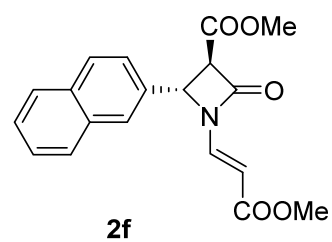

( \pm ) Methyl (2S,3R)-1-((E)-3-methoxy-3-oxoprop-1-en-1-yl)-2-(naphthalen-2-yl)-4-oxoazetidine-3-carboxylate (2f). According to the general procedure, $\beta$-lactam $2 \mathbf{f}$ was obtained from 2-naphthaldehyde oxime $(51.3 \mathrm{mg}, 0.3 \mathrm{mmol})$ and methyl propiolate $(76.5 \mathrm{mg}, 0.9 \mathrm{mmol})$ as light yellow oil $(69.9 \mathrm{mg}, 69 \%$ yield, $94 / 6 \mathrm{dr}$; eluent: $\mathrm{PE} / \mathrm{EtOAc}=8: 1$ to 4:1). ${ }^{1} \mathrm{H}$ NMR (400 MHz, $\left.\mathrm{CDCl}_{3}\right) \delta: 7.92-7.89(\mathrm{~d}, J=8.5 \mathrm{~Hz}, 1 \mathrm{H}), 7.87-7.83(\mathrm{~m}, 2 \mathrm{H}), 7.82(\mathrm{~s}, 1 \mathrm{H}), 7.75-7.71(\mathrm{~d}, J=$ $14.2 \mathrm{~Hz}, 1 \mathrm{H}), 7.55-7.53(\mathrm{~m}, 2 \mathrm{H}), 7.38-7.35(\mathrm{dd}, J=8.5,1.8 \mathrm{~Hz}, 1 \mathrm{H}), 5.36(\mathrm{~d}, J=3.0 \mathrm{~Hz}, 1 \mathrm{H}), 5.19-5.15$ (d, $J=14.2$ $\mathrm{Hz}, 1 \mathrm{H}), 4.13-4.12(\mathrm{~d}, J=3.1 \mathrm{~Hz}, 1 \mathrm{H}), 3.84(\mathrm{~s}, 3 \mathrm{H}), 3.63$ (s, 3H) ppm; ${ }^{13} \mathrm{C} \mathrm{NMR}\left(100 \mathrm{MHz}, \mathrm{CDCl}_{3}\right) \delta: 166.7,165.8$, $159.5,133.7,133.6,133.1,131.7,129.7,128.0,127.8,127.0,126.9,126.3,122.4,102.9,64.2,58.8,53.1,51.5 \mathrm{ppm}$; IR (Neat): 2952, 1780, 1708, 1629, 1398, 1251, 1162, 1033, 960, 908, 839, 727, 648, $477 \mathrm{~cm}^{-1}$; HRMS (ESI) $\mathrm{m} / \mathrm{z}$ : $[\mathrm{M}+\mathrm{H}]^{+}$calcd. for $\mathrm{C}_{19} \mathrm{H}_{18} \mathrm{NO}_{5}$ : 340.1179; Found: 340.1179 .

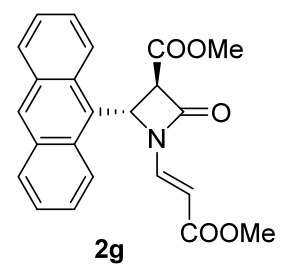

( \pm ) Methyl (2S,3R)-2-(anthracen-9-yl)-1-((E)-3-methoxy-3-oxoprop-1-en-1-yl)-4-oxoazetidine-3-carboxylate (2g). According to the general procedure, $\beta$-lactam $2 \mathbf{g}$ was obtained from anthracene-9-carbaldehyde oxime ( $66.3 \mathrm{mg}, 0.3$ $\mathrm{mmol})$ and methyl propiolate $(76.5 \mathrm{mg}, 0.9 \mathrm{mmol})$ as light yellow solid $(68.8 \mathrm{mg}, 59 \%$ yield, $97 / 3 \mathrm{dr}$; eluent: PE/EtOAc $=6: 1$ to 4:1). ${ }^{1} \mathrm{H}$ NMR $\left(400 \mathrm{MHz}, \mathrm{CDCl}_{3}\right) \delta: 8.47(\mathrm{~s}, 1 \mathrm{H}), 8.22(\mathrm{~s}, 2 \mathrm{H}), 7.80-7.97(\mathrm{~d}, J=8.4 \mathrm{~Hz}, 2 \mathrm{H}), 7.64-$ $7.60(\mathrm{~d}, J=14.2 \mathrm{~Hz}, 1 \mathrm{H}), 7.53-7.49$ (t, $J=7.5 \mathrm{~Hz}, 2 \mathrm{H}), 7.45-7.41(\mathrm{t}, J=7.4 \mathrm{~Hz}, 2 \mathrm{H}), 6.76-6.75(\mathrm{~d}, J=3.8 \mathrm{~Hz}, 1 \mathrm{H})$, 4.82-4.78 (d, $J=14.2 \mathrm{~Hz}, 1 \mathrm{H}), 4.71-4.70(\mathrm{~d}, J=3.8 \mathrm{~Hz}, 1 \mathrm{H}), 3.77$ (s, 3H), $3.43(\mathrm{~s}, 3 \mathrm{H}) \mathrm{ppm} ;{ }^{13} \mathrm{C}$ NMR $(100 \mathrm{MHz}$, $\left.\mathrm{CDCl}_{3}\right) \delta: 166.7,166.3,160.2,133.9,131.4,130.9,130.1,127.8,125.2,122.1,121.7,102.3,62.4,54.9,53.2,51.4$ ppm; IR (Neat): 2952, 2255, 1779, 1708, 1629, 1437, 1272, 1165, 906, 838, 723, 647, $523 \mathrm{~cm}^{-1}$; HRMS (ESI) $m / z$ : $[\mathrm{M}+\mathrm{H}]^{+}$calcd. for $\mathrm{C}_{23} \mathrm{H}_{20} \mathrm{NO}_{5}$ : 390.1336; Found: 390.1334 . 


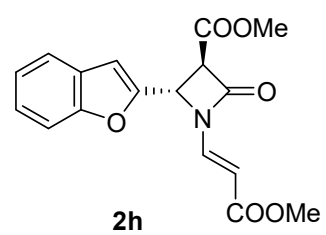

( \pm ) Methyl (2S,3R)-2-(benzofuran-2-yl)-1-((E)-3-methoxy-3-oxoprop-1-en-1-yl)-4-oxoazetidine-3-carboxylate (2h). According to the general procedure, $\beta$-lactam $\mathbf{2 h}$ was obtained from benzofuran-2-carbaldehyde oxime (48.3 mg, $0.3 \mathrm{mmol})$ and methyl propiolate $(76.5 \mathrm{mg}, 0.9 \mathrm{mmol})$ as light yellow oil $(68.0 \mathrm{mg}$, 69\% yield, 99/1 dr; eluent: $\mathrm{PE} / \mathrm{EtOAc}=8: 1$ to 4:1). ${ }^{1} \mathrm{H} \mathrm{NMR}\left(400 \mathrm{MHz} \mathrm{CDCl}_{3}\right) \delta: 7.64-7.60(\mathrm{~d}, J=14.3 \mathrm{~Hz}, 1 \mathrm{H}), 7.60-7.57$ (m, $\left.1 \mathrm{H}\right), 7.49-7.46$ (m, 1H), 7.37-7.33 (m, 1H), 7.29-7.25 (m, 1H), 6.94 (d, J=0.7 Hz, 1H), 5.45-5.42 (d, J = 14.3 Hz, 1H), 5.37-5.36 (d, J $=3.0 \mathrm{~Hz}, 1 \mathrm{H}), 4.54-4.53(\mathrm{~d}, J=3.1 \mathrm{~Hz}, 1 \mathrm{H}), 3.84(\mathrm{~s}, 3 \mathrm{H}), 3.66(\mathrm{~s}, 3 \mathrm{H}) \mathrm{ppm} ;{ }^{13} \mathrm{C} \mathrm{NMR}\left(100 \mathrm{MHz}, \mathrm{CDCl}_{3}\right) \delta: 166.7$, $165.5,159.0,155.2,148.8,133.7,127.2,125.7,123.5,121.5,111.5,108.4,102.5,60.9,53.2,52.0,51.5$ ppm; IR (Neat): 2953, 1785, 1711, 1632, 1454, 1280, 1165, 954, 840, $752 \mathrm{~cm}^{-1}$; HRMS (ESI) $m / z$ : [M+H] calcd. for $\mathrm{C}_{17} \mathrm{H}_{16} \mathrm{NO}_{6}$ : 330.0972; Found: 330.0975 .

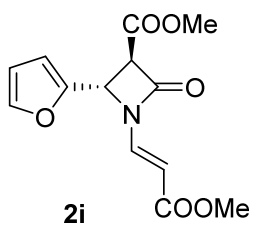

( \pm ) Methyl (2S,3R)-2-(furan-2-yl)-1-((E)-3-methoxy-3-oxoprop-1-en-1-yl)-4-oxoazetidine-3-carboxylate (2i). According to the general procedure, $\beta$-lactam $\mathbf{2 i}$ was obtained from furan-2-carbaldehyde oxime $(33.3 \mathrm{mg}, 0.3 \mathrm{mmol})$ and methyl propiolate $(76.5 \mathrm{mg}, 0.9 \mathrm{mmol})$ as light yellow oil $(61.2 \mathrm{mg}, 73 \%$ yield, 93/7 dr; eluent: PE/EtOAc $=8: 1$ to 4:1). ${ }^{1} \mathrm{H}$ NMR $\left(400 \mathrm{MHz} \mathrm{CDCl}_{3}\right) \delta: 7.59-7.55(\mathrm{~d}, J=14.3 \mathrm{~Hz}, 1 \mathrm{H}), 7.46(\mathrm{~d}, J=14.4 \mathrm{~Hz}, 1 \mathrm{H}), 6.56-6.55(\mathrm{~d}, J=3.3 \mathrm{~Hz}$, $1 \mathrm{H}), 6.42-6.40(\mathrm{~m}, 1 \mathrm{H}), 5.40-5.37(\mathrm{~d}, J=14.3 \mathrm{~Hz}, 1 \mathrm{H}), 5.24-5.23(\mathrm{~d}, J=3.1 \mathrm{~Hz}, 1 \mathrm{H}), 4.41(\mathrm{~d}, J=3.1 \mathrm{~Hz}, 1 \mathrm{H}), 3.84(\mathrm{~s}$, 3H), 3.69 (s, 3H) ppm; ${ }^{13} \mathrm{C}$ NMR $\left(100 \mathrm{MHz}, \mathrm{CDCl}_{3}\right) \delta: 166.8,165.6,159.2,146.6,144.2,133.8,111.6,110.9,102.4$, 60.8, 53.2, 51.7, 51.6 ppm; IR (Neat): 2953, 1783, 1735, 1630, 1438, 1252, 1163, 960, 841, 745, 597 cm ${ }^{-1}$, HRMS (ESI) $m / z:[\mathrm{M}+\mathrm{H}]^{+}$calcd. for $\mathrm{C}_{13} \mathrm{H}_{14} \mathrm{NO}_{6}: 280.0816$; Found: 280.0815 .

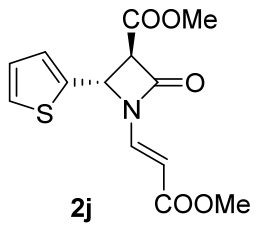

( \pm ) Methyl (3R,4S)-1-((E)-3-methoxy-3-oxoprop-1-en-1-yl)-2-oxo-4-(thiophen-2-yl)azetidine-3-carboxylate (2j). According to the general procedure, $\beta$-lactam $\mathbf{2} \mathbf{j}$ was obtained from thiophene-2-carbaldehyde oxime (38.1 mg, 0.3 mmol) and methyl propiolate (76.5 mg, $0.9 \mathrm{mmol})$ as light yellow oil (65.6 mg, 74\% yield, 94/6 dr; eluent: PE/EtOAc $=10: 1$ to 4:1). ${ }^{1} \mathrm{H}$ NMR (400 MHz, $\left.\mathrm{CDCl}_{3}\right) \delta: 7.61-7.57(\mathrm{~d}, J=14.3 \mathrm{~Hz}, 1 \mathrm{H}), 7.39-7.37(\mathrm{~d}, J=5.0 \mathrm{~Hz}, 1 \mathrm{H}), 7.18-7.17$ $(\mathrm{d}, J=3.4 \mathrm{~Hz}, 1 \mathrm{H}), 7.04-7.02(\mathrm{~m}, 1 \mathrm{H}), 5.48-5.47(\mathrm{~d}, J=3.0 \mathrm{~Hz}, 1 \mathrm{H}), 5.35-5.32(\mathrm{~d}, J=14.3 \mathrm{~Hz}, 1 \mathrm{H}), 4.19-4.18(\mathrm{~d}, J=$ $3.0 \mathrm{~Hz}, 1 \mathrm{H}), 3.83(\mathrm{~s}, 3 \mathrm{H}), 3.69$ (s, 3H) ppm; ${ }^{13} \mathrm{C} \mathrm{NMR}\left(100 \mathrm{MHz}, \mathrm{CDCl}_{3}\right) \delta: 166.7,165.5,159.1,137.7,133.5,127.6$, 127.5, 127.1, 103.1, 65.1, 54.5, 53.2, 51.6 ppm; IR (Neat): 2953, 1784, 1711, 1633, 1437, 1283, 1166, 961, 840, 711, $496 \mathrm{~cm}^{-1}$; HRMS (ESI) m/z: [M+H] $]^{+}$calcd. for $\mathrm{C}_{13} \mathrm{H}_{14} \mathrm{NO}_{5} \mathrm{~S}: 296.0587$; Found: 296.0586 . 


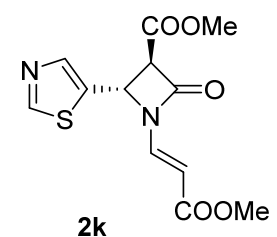

( \pm ) Methyl (3R,4S)-1-((E)-3-methoxy-3-oxoprop-1-en-1-yl)-2-oxo-4-(thiazol-5-yl)azetidine-3-carboxylate (2k). According to the general procedure, $\beta$-lactam $2 \mathbf{k}$ was obtained from thiazole-5-carbaldehyde oxime $(38.4 \mathrm{mg}, 0.3$ $\mathrm{mmol})$ and methyl propiolate $(76.5 \mathrm{mg}, 0.9 \mathrm{mmol})$ as light yellow oil (62.2 $\mathrm{mg}, 70 \%$ yield, 10/1 dr; eluent: PE/EtOAc $=8: 1$ to $4: 1) .{ }^{1} \mathrm{H}$ NMR $\left(400 \mathrm{MHz}, \mathrm{CDCl}_{3}\right) \delta: 8.91(\mathrm{~s}, 1 \mathrm{H}), 8.01(\mathrm{~s}, 1 \mathrm{H}), 7.61-7.57(\mathrm{~d}, J=14.3 \mathrm{~Hz}, 1 \mathrm{H}), 5.61-5.60(\mathrm{~d}, J$ $=3.0 \mathrm{~Hz}, 1 \mathrm{H}), 5.33-5.29(\mathrm{~d}, J=14.3 \mathrm{~Hz}, 1 \mathrm{H}), 4.20-4.19(\mathrm{~d}, J=3.0 \mathrm{~Hz}, 1 \mathrm{H}), 3.85(\mathrm{~s}, 3 \mathrm{H}), 3.70(\mathrm{~s}, 3 \mathrm{H}) \mathrm{ppm} ;{ }^{13} \mathrm{C} \mathrm{NMR}$ $\left(100 \mathrm{MHz}, \mathrm{CDCl}_{3}\right) \delta: 166.4,165.0,158.4,154.8,143.4,133.1,103.3,64.9,53.3,52.1,51.6$ ppm; IR (Neat): 3086, 2953, 1786, 1708, 1633, 1438, 1290, 1167, 963, 841, $605 \mathrm{~cm}^{-1}$; HRMS (ESI) m/z: $[\mathrm{M}+\mathrm{H}]^{+}$calcd. for $\mathrm{C}_{12} \mathrm{H}_{13} \mathrm{~N}_{2} \mathrm{O}_{5} \mathrm{~S}$ : 297.0540; Found: 297.0542.

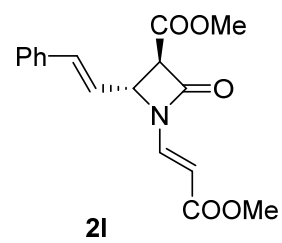

( \pm ) Methyl (3R,4R)-1-((E)-3-methoxy-3-oxoprop-1-en-1-yl)-2-oxo-4-((E)-styryl)azetidine-3-carboxylate (21). According to the general procedure, $\beta$-lactam 21 was obtained from (2E)-cinnamaldehyde oxime $(44.1 \mathrm{mg}, 0.3 \mathrm{mmol})$ and methyl propiolate $(76.5 \mathrm{mg}, 0.9 \mathrm{mmol})$ as light yellow oil $(67.4 \mathrm{mg}, 71 \%$ yield, $87 / 13 \mathrm{dr}$; eluent: PE/EtOAc $=8: 1$ to 4:1). ${ }^{1} \mathrm{H} \mathrm{NMR}\left(400 \mathrm{MHz}, \mathrm{CDCl}_{3}\right) \delta: 7.58-7.55(\mathrm{~d}, J=14.3 \mathrm{~Hz}, 1 \mathrm{H}), 7.34-7.27(\mathrm{~m}, 5 \mathrm{H}), 6.80-6.76(\mathrm{~d}, J=15.8 \mathrm{~Hz}$, $1 \mathrm{H}), 6.12-6.06(\mathrm{~m}, 1 \mathrm{H}), 5.46-5.43(\mathrm{~d}, J=14.3 \mathrm{~Hz}, 1 \mathrm{H}), 4.79-4.76(\mathrm{~m}, 1 \mathrm{H}), 3.96-3.95(\mathrm{~d}, J=3.0 \mathrm{~Hz}, 1 \mathrm{H}), 3.76(\mathrm{~s}, 3 \mathrm{H})$, 3.63 (s, 3H) ppm; ${ }^{13} \mathrm{C}$ NMR (100 MHz, $\left.\mathrm{CDCl}_{3}\right) \delta: 167.0,165.9,159.2,136.9,134.8,134.2,129.1,128.9,126.9,122.8$, 102.7, 62.0, 58.0, 53.2, 51.6 ppm; IR (Neat): 2952, 2154, 1979, 1784, 1735, 1633, 1437, 1288, 1168, 969, 840, 749, $694 \mathrm{~cm}^{-1}$; HRMS (ESI) m/z: [M+H] $]^{+}$calcd. for $\mathrm{C}_{17} \mathrm{H}_{18} \mathrm{NO}_{5}$ : 316.1179 ; Found: 316.1173 .

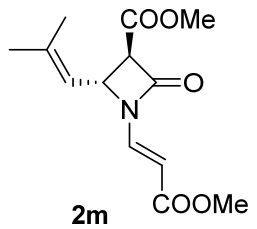

( \pm ) Methyl (2R,3R)-1-((E)-3-methoxy-3-oxoprop-1-en-1-yl)-2-(2-methylprop-1-en-1-yl)-4-oxoazetidine-3carboxylate (2m). According to the general procedure, $\beta$-lactam $\mathbf{2 m}$ was obtained from 3-methylbut-2-enal oxime (30.0 mg, $0.3 \mathrm{mmol}$ ) and methyl propiolate $(76.5 \mathrm{mg}, 0.9 \mathrm{mmol})$ as light yellow oil (57.1 $\mathrm{mg}, 71 \%$ yield, 88/12 dr; eluent: $\mathrm{PE} / \mathrm{EtOAc}=10: 1$ to 4:1). ${ }^{1} \mathrm{H} \mathrm{NMR}\left(400 \mathrm{MHz}, \mathrm{CDCl}_{3}\right) \delta: 7.50-7.47(\mathrm{~d}, J=14.2 \mathrm{~Hz}, 1 \mathrm{H}), 5.30-5.27(\mathrm{~d}, J=14.2$ $\mathrm{Hz}, 1 \mathrm{H}), 5.12-5.09$ (m, 1H), 4.86-4.83 (m, 1H), 3.80-3.79 (d, J=3.0 Hz, 1H), 3.74 (s, 3H), 3.65 (s, 3H), 1.77-1.76 (d, $J$ $=1.1 \mathrm{~Hz}, 3 \mathrm{H}), 1.74(\mathrm{~s}, 3 \mathrm{H}) \mathrm{ppm} ;{ }^{13} \mathrm{C} \mathrm{NMR}\left(100 \mathrm{MHz}, \mathrm{CDCl}_{3}\right) \delta: 167.1,166.1,159.6,142.1,134.0,119.2,101.9,61.7$, 53.9, 53.0, 51.5, 25.8, 18.5 ppm; IR (Neat): 2953, 1781, 1711, 1629, 1438, 1279, 1162, 967, $836 \mathrm{~cm}^{-1}$; HRMS (ESI) $m / z:[\mathrm{M}+\mathrm{H}]^{+}$calcd. for $\mathrm{C}_{13} \mathrm{H}_{18} \mathrm{NO}_{5}: 268.1179$; Found: 268.1184 . 


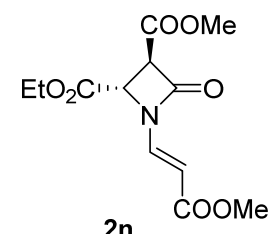

( \pm ) 2-Ethyl 3-methyl (2S,3R)-1-((E)-3-methoxy-3-oxoprop-1-en-1-yl)-4-oxoazetidine-2,3-dicarboxylate (2n). According to the general procedure, $\beta$-lactam 2n was obtained from ethyl 2-(hydroxyimino)acetate (35.1 mg, 0.3 $\mathrm{mmol})$ and methyl propiolate $(76.5 \mathrm{mg}, 0.9 \mathrm{mmol})$ as light yellow oil (39.3 mg, 46\% yield, 86/14 dr; eluent: PE/EtOAc $=2: 1$ to $1: 1) .{ }^{1} \mathrm{H}$ NMR $\left(400 \mathrm{MHz}, \mathrm{CDCl}_{3}\right) \delta: 7.55-7.51(\mathrm{~d}, J=14.2 \mathrm{~Hz}, 1 \mathrm{H}), 5.47-5.43(\mathrm{~d}, J=14.2 \mathrm{~Hz}, 1 \mathrm{H}), 4.64-4.63$ $(\mathrm{d}, J=3.0 \mathrm{~Hz}, 1 \mathrm{H}), 4.26-4.22(\mathrm{~m}, 3 \mathrm{H}), 3.77(\mathrm{~s}, 3 \mathrm{H}), 3.67(\mathrm{~s}, 3 \mathrm{H}), 1.28-1.25(\mathrm{~m}, 3 \mathrm{H}) \mathrm{ppm} ;{ }^{13} \mathrm{C} \mathrm{NMR}(100 \mathrm{MHz}$ $\left.\mathrm{CDCl}_{3}\right) \delta: 167.0,166.7,164.9,158.2,133.8,103.2,62.9,59.0,54.6,53.4,51.7,14.1$ ppm; IR (Neat): 3270, 2955, 1795, 1717, 1636, 1438, 1258, 1171, 1072, 966, 848, $768 \mathrm{~cm}^{-1}$; HRMS (ESI) m/z: [M+H] calcd. for $\mathrm{C}_{12} \mathrm{H}_{16} \mathrm{NO}_{7}: 286.0921$; Found: 286.0926.

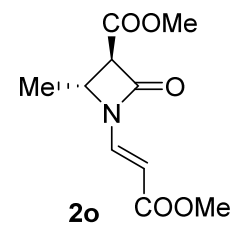

( \pm ) Methyl (2R,3R)-1-((E)-3-methoxy-3-oxoprop-1-en-1-yl)-2-methyl-4-oxoazetidine-3-carboxylate

(20).

According to the general procedure, $\beta$-lactam 20 was obtained from acetaldehyde oxime $(17.8 \mathrm{mg}, 0.3 \mathrm{mmol})$ and methyl propiolate $(76.5 \mathrm{mg}, 0.9 \mathrm{mmol})$ as light yellow oil $(54.9 \mathrm{mg}, 80.6 \%$ yield, 96/4 dr; eluent: PE/EtOAc $=6: 1$ to 4:1). ${ }^{1} \mathrm{H} \mathrm{NMR}\left(400 \mathrm{MHz} \mathrm{CDCl}_{3}\right) \delta: 7.52-7.48(\mathrm{~d}, J=14.4 \mathrm{~Hz}, 1 \mathrm{H}), 5.43-5.40(\mathrm{~d}, J=14.4 \mathrm{~Hz}, 1 \mathrm{H}), 4.27-4.23$ (m, 1H), $3.73(\mathrm{~s}, 4 \mathrm{H}), 3.67(\mathrm{~s}, 3 \mathrm{H}), 1.49-1.47(\mathrm{~d}, J=6.2 \mathrm{~Hz}, 3 \mathrm{H}) \mathrm{ppm} ;{ }^{13} \mathrm{C} \mathrm{NMR}\left(100 \mathrm{MHz}, \mathrm{CDCl}_{3}\right) \delta: 167.0,166.1,159.2$, 133.9, 101.9, 61.8, 52.9, 52.3, 51.5, 17.2 ppm; IR (Neat): 2954, 1777, 1732, 1628, 1438, 1289, 1157, 963, 838, 730, $555 \mathrm{~cm}^{-1}$; HRMS (ESI) $\mathrm{m} / z$ : [M+H] $]^{+}$calcd. for $\mathrm{C}_{10} \mathrm{H}_{14} \mathrm{NO}_{5}: 228.0866$; Found: 228.0862 .<smiles>COC(=O)/C=C/N1C(=O)C(C(C)=O)[C@H]1Cc1ccccc1</smiles>

( \pm ) Methyl (2R,3R)-2-benzyl-1-((E)-3-methoxy-3-oxoprop-1-en-1-yl)-4-oxoazetidine-3-carboxylate (2p). According to the general procedure, $\beta$-lactam 2p was obtained from 2-phenylacetaldehyde oxime (40.5 mg, $0.3 \mathrm{mmol})$ and methyl propiolate $(76.5 \mathrm{mg}, 0.9 \mathrm{mmol})$ as light yellow oil $(71.3 \mathrm{mg}, 78 \%$ yield, 94/6 dr; eluent: PE/EtOAc = 10:1 to 4:1). ${ }^{1} \mathrm{H}$ NMR $\left(400 \mathrm{MHz}, \mathrm{CDCl}_{3}\right) \delta: 7.48-7.44$ (d, $\left.J=14.4 \mathrm{~Hz}, 1 \mathrm{H}\right), 7.28-7.20$ (m, 3H), 7.10-7.08 (m, 2H), 5.49$5.45(\mathrm{~d}, J=14.4 \mathrm{~Hz}, 1 \mathrm{H}), 4.48-4.44(\mathrm{~m}, 1 \mathrm{H}), 3.77(\mathrm{~d}, J=3.0 \mathrm{~Hz}, 1 \mathrm{H}), 3.67(\mathrm{~s}, 3 \mathrm{H}), 3.66(\mathrm{~s}, 3 \mathrm{H}), 3.19-2.96(\mathrm{~m}, 2 \mathrm{H})$ ppm; ${ }^{13} \mathrm{C}$ NMR $\left(100 \mathrm{MHz}, \mathrm{CDCl}_{3}\right) \delta: 167.0,166.0,159.2,134.3,134.2,129.2,129.0,128.9,127.6,102.6,59.2,56.6$, 52.9, 51.6, 36.7 ppm; IR (Neat): 2952, 1777, 1732, 1629, 1497, 1251, 1163, 964, 842, 701, $506 \mathrm{~cm}^{-1}$; HRMS (ESI) $m / z:[\mathrm{M}+\mathrm{H}]^{+}$calcd. for $\mathrm{C}_{16} \mathrm{H}_{18} \mathrm{NO}_{5}$ : 304.1179; Found: 304.1182 .

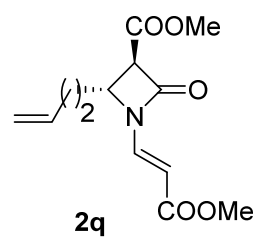


( \pm ) Methyl (2R,3R)-2-(but-3-en-1-yl)-1-((E)-3-methoxy-3-oxoprop-1-en-1-yl)-4-oxoazetidine-3-carboxylate (2q). According to the general procedure, $\beta$-lactam $2 \mathbf{q}$ was obtained from pent-4-enal oxime $(30.1 \mathrm{mg}, 0.3 \mathrm{mmol})$ and methyl propiolate $(76.5 \mathrm{mg}, 0.9 \mathrm{mmol})$ as light yellow oil $(50.5 \mathrm{mg}, 63 \%$ yield, $93 / 7 \mathrm{dr}$; eluent: $\mathrm{PE} / \mathrm{EtOAc}=10: 1$ to 4:1). ${ }^{1} \mathrm{H}$ NMR (400 MHz, $\left.\mathrm{CDCl}_{3}\right) \delta: 7.58-7.54(\mathrm{~d}, J=14.3 \mathrm{~Hz}, 1 \mathrm{H}), 5.83-5.74(\mathrm{~m}, 1 \mathrm{H}), 5.56-5.52(\mathrm{~d}, J=14.3 \mathrm{~Hz}, 1 \mathrm{H})$, 5.10-5.07 (d, $J=51.4 \mathrm{~Hz}, 1 \mathrm{H}), 5.06-5.04(\mathrm{~m}, 1 \mathrm{H}), 4.26-4.22(\mathrm{~m}, 1 \mathrm{H}), 3.86(\mathrm{~d}, J=3.0 \mathrm{~Hz}, 1 \mathrm{H}), 3.80(\mathrm{~s}, 3 \mathrm{H}), 3.74(\mathrm{~s}$, $3 \mathrm{H}), 2.24-2.15(\mathrm{~m}, 3 \mathrm{H}), 1.75-1.68(\mathrm{~m}, 1 \mathrm{H}) \mathrm{ppm} ;{ }^{13} \mathrm{C} \mathrm{NMR}\left(100 \mathrm{MHz}, \mathrm{CDCl}_{3}\right) \delta: 167.0,166.2,159.3,135.9,134.1$, 116.5, 102.2, 60.2, 56.2, 52.9, 51.5, 30.0, 29.0 ppm; IR (Neat): 2953, 1780, 1722, 1629, 1432, 1279, 1163, 964, 842 $\mathrm{cm}^{-1}$; HRMS (ESI) $\mathrm{m} / z$ : $[\mathrm{M}+\mathrm{H}]^{+}$calcd. for $\mathrm{C}_{13} \mathrm{H}_{18} \mathrm{NO}_{5}$ : 268.1179; Found: 268.1176 .

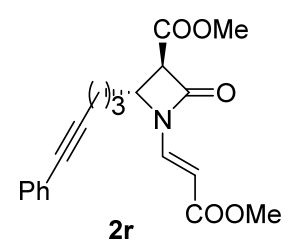

( \pm Methyl (3R,4R)-1-((E)-3-methoxy-3-oxoprop-1-en-1-yl)-2-oxo-4-(5-phenylpent-4-yn-1-yl)azetidine-3carboxylate (2r). According to the general procedure, $\beta$-lactam $2 \mathbf{r}$ was obtained from 6-phenylhex-5-ynal oxime (56.1 $\mathrm{mg}, 0.3 \mathrm{mmol})$ and methyl propiolate $(76.5 \mathrm{mg}, 0.9 \mathrm{mmol})$ as light yellow oil $(73.3 \mathrm{mg}, 69 \%$ yield, $97 / 3 \mathrm{dr}$; eluent: $\mathrm{PE} / \mathrm{EtOAc}=8: 1$ to 4:1). ${ }^{1} \mathrm{H}$ NMR $\left(400 \mathrm{MHz}, \mathrm{CDCl}_{3}\right) \delta: 7.52-7.49(\mathrm{~d}, J=14.3 \mathrm{~Hz}, 1 \mathrm{H}), 7.33-7.31(\mathrm{~m}, 2 \mathrm{H}), 7.22-7.20$ $(\mathrm{m}, 3 \mathrm{H}), 5.51-5.48(\mathrm{~d}, J=14.4 \mathrm{~Hz}, 1 \mathrm{H}), 4.24-4.20(\mathrm{~m}, 1 \mathrm{H}), 3.82(\mathrm{~d}, J=3.0 \mathrm{~Hz}, 1 \mathrm{H}), 3.72(\mathrm{~s}, 3 \mathrm{H}), 3.65(\mathrm{~s}, 3 \mathrm{H}), 2.46-$ $2.42(\mathrm{t}, J=3.7 \mathrm{~Hz}, 2 \mathrm{H}), 2.24-2.18(\mathrm{~m}, 1 \mathrm{H}), 1.80-1.68(\mathrm{~m}, 1 \mathrm{H}), 1.64-1.59(\mathrm{~m}, 2 \mathrm{H}) \mathrm{ppm} ;{ }^{13} \mathrm{C} \mathrm{NMR}\left(100 \mathrm{MHz}, \mathrm{CDCl}_{3}\right)$ $\delta: 167.0,166.2,159.3,134.1,131.5,128.2,127.8,123.3,102.3,88.1,81.9,60.0,56.1,53.0,51.5,29.8,23.7,18.9$ ppm; IR (Neat): 2954, 1781, 1712, 1633, 1497, 1261, 1158, 967, 844, 721, $532 \mathrm{~cm}^{-1}$; HRMS (ESI) $\mathrm{m} / z$ : [M+H] $]^{+}$calcd. for $\mathrm{C}_{20} \mathrm{H}_{22} \mathrm{NO}_{5}$ : 356.1492; Found: 356.1490 .

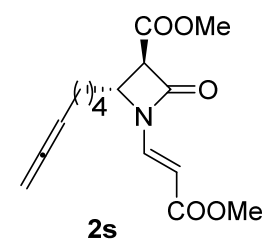

( \pm ) Methyl (3R)-2-(hepta-5,6-dien-1-yl)-1-((E)-3-methoxy-3-oxoprop-1-en-1-yl)-4-oxoazetidine-3-carboxylate (2s). According to the general procedure, $\beta$-lactam $2 \mathrm{~s}$ was obtained from octa-6,7-dienal oxime (41.7 $\mathrm{mg}, 0.3 \mathrm{mmol})$ and methyl propiolate $(76.5 \mathrm{mg}, 0.9 \mathrm{mmol})$ as light yellow oil $(62.1 \mathrm{mg}, 67 \%$ yield, $91 / 9 \mathrm{dr}$; eluent: $\mathrm{PE} / \mathrm{EtOAc}=8: 1$ to 4:1). ${ }^{1} \mathrm{H}$ NMR (400 MHz, $\left.\mathrm{CDCl}_{3}\right) \delta: 7.58-7.54(\mathrm{~d}, J=14.3 \mathrm{~Hz}, 1 \mathrm{H}), 5.55-5.51(\mathrm{~d}, J=14.3 \mathrm{~Hz}, 1 \mathrm{H}), 5.11-5.05(\mathrm{~m}, 1 \mathrm{H})$, 4.69-4.66 (m, 2H), 4.24-4.20 (m, 1H), $3.82(\mathrm{~d}, J=3.1 \mathrm{~Hz}, 1 \mathrm{H}), 3.81$ (s, 3H), $3.74(\mathrm{~s}, 3 \mathrm{H}), 2.13-2.07$ (m, 1H), 2.06-1.99 $(\mathrm{m}, 2 \mathrm{H}), 1.65-1.57(\mathrm{~m}, 1 \mathrm{H}), 1.52-1.38(\mathrm{~m}, 4 \mathrm{H}) \mathrm{ppm} ;{ }^{13} \mathrm{C} \mathrm{NMR}\left(100 \mathrm{MHz}, \mathrm{CDCl}_{3}\right) \delta: 208.6,167.1,166.4,159.5,134.2$, 102.3, 89.3, 60.1, 56.6, 53.0, 51.6, 30.8, 28.4, 27.7, 24.0 ppm; IR (Neat): 2949, 2858, 1954, 1780, 1711, 1629, 1437, 1292, 1164, 967, $843 \mathrm{~cm}^{-1}$; HRMS (ESI) $\mathrm{m} / z$ : $[\mathrm{M}+\mathrm{H}]^{+}$calcd. for $\mathrm{C}_{16} \mathrm{H}_{22} \mathrm{NO}_{5}: 308.1492$; Found: 308.1494 .

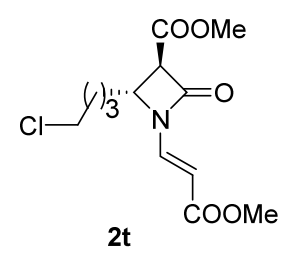

( \pm ) Methyl (2R,3R)-2-(4-chlorobutyl)-1-((E)-3-methoxy-3-oxoprop-1-en-1-yl)-4-oxoazetidine-3-carboxylate (2t). According to the general procedure, $\beta$-lactam $2 \mathbf{t}$ was obtained from 5-chloropentanal oxime ( $40.5 \mathrm{mg}, 0.3 \mathrm{mmol})$ and 
methyl propiolate $(76.5 \mathrm{mg}, 0.9 \mathrm{mmol})$ as light yellow oil $(63.8 \mathrm{mg}, 70 \%$ yield, $91 / 9 \mathrm{dr}$; eluent: $\mathrm{PE} / \mathrm{EtOAc}=6: 1$ to 3:1). ${ }^{1} \mathrm{H}$ NMR (400 MHz, $\left.\mathrm{CDCl}_{3}\right) \delta: 7.50-7.47(\mathrm{~d}, J=14.4 \mathrm{~Hz}, 1 \mathrm{H}), 5.48-5.45(\mathrm{~d}, J=14.3 \mathrm{~Hz}, 1 \mathrm{H}), 4.19-4.15(\mathrm{~m}, 1 \mathrm{H})$, $3.79-3.78(\mathrm{~d}, J=3.0 \mathrm{~Hz}, 1 \mathrm{H}), 3.74(\mathrm{~s}, 3 \mathrm{H}), 3.67(\mathrm{~s}, 3 \mathrm{H}), 3.51-3.48(\mathrm{~m}, 2 \mathrm{H}), 2.09-2.01(\mathrm{~m}, 1 \mathrm{H}), 1.80-1.74(\mathrm{~m}, 2 \mathrm{H})$, 1.63-1.54 (m, 1H), 1.52-1.43 (m, 2H) ppm; ${ }^{13} \mathrm{C}$ NMR (100 MHz, $\left.\mathrm{CDCl}_{3}\right) \delta: 167.0,166.1,159.3,134.1,102.3,59.9$, 56.3, 53.0, 51.6, 44.2, 31.8, 30.2, 21.9 ppm; IR (Neat): 2952, 2865, 1779, 1711, 1629, 1437, 1292, 1165, 967, 843, 735 $\mathrm{cm}^{-1}$; HRMS (ESI) m/z: [M+H] $]^{+}$calcd. for $\mathrm{C}_{13} \mathrm{H}_{19} \mathrm{ClNO}_{5}$ : 304.0946; Found: 304.0942.

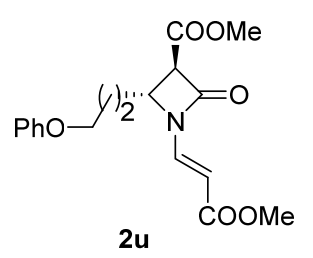

( \pm Methyl (3R,4R)-1-((E)-3-methoxy-3-oxoprop-1-en-1-yl)-2-oxo-4-(3-phenoxypropyl)azetidine-3-carboxylate (2u). According to the general procedure, $\beta$-lactam $2 \mathbf{u}$ was obtained from 4-phenoxybutanal oxime (53.7 $\mathrm{mg}, 0.3$ $\mathrm{mmol}$ ) and methyl propiolate $(76.5 \mathrm{mg}, 0.9 \mathrm{mmol})$ as light yellow oil $(73.2 \mathrm{mg}, 70 \%$ yield, 94/6 dr; eluent: PE/EtOAc $=8: 1$ to 3:1). ${ }^{1} \mathrm{H}$ NMR $\left(400 \mathrm{MHz}, \mathrm{CDCl}_{3}\right) \delta: 7.60-7.56(\mathrm{~d}, J=14.4 \mathrm{~Hz}, 1 \mathrm{H}), 7.31-7.28(\mathrm{~m}, 2 \mathrm{H}), 6.98-6.95(\mathrm{~m}, 1 \mathrm{H})$, 6.90-6.88 (m, 2H), 5.60-5.56 (d, $J=14.4 \mathrm{~Hz}, 1 \mathrm{H}), 4.36-4.32(\mathrm{~m}, 1 \mathrm{H}), 4.03-4.00(\mathrm{~m}, 2 \mathrm{H}), 3.91-3.90(\mathrm{~d}, J=3.0 \mathrm{~Hz}, 1 \mathrm{H})$, $3.79(\mathrm{~s}, 3 \mathrm{H}), 3.74(\mathrm{~s}, 3 \mathrm{H}), 2.35-2.28(\mathrm{~m}, 1 \mathrm{H}), 1.90-1.81(\mathrm{~m}, 3 \mathrm{H}) \mathrm{ppm} ;{ }^{13} \mathrm{C} \mathrm{NMR}\left(100 \mathrm{MHz}, \mathrm{CDCl}_{3}\right) \delta: 167.0,166.2$, 159.3, 158.4, 134.0, 129.4, 120.9, 114.3, 102.3, 66.4, 60.1, 56.2, 52.9, 51.5, 27.8, 24.6 ppm; IR (Neat): 2953, 1787, $1734,1625,1500,1249,1164,964,843,712,513 \mathrm{~cm}^{-1}$; HRMS (ESI) $m / z:[\mathrm{M}+\mathrm{H}]^{+}$calcd. for $\mathrm{C}_{18} \mathrm{H}_{22} \mathrm{NO}_{6}: 348.1442$; Found: 348.1451.

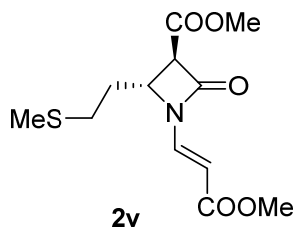

( \pm ) Methyl (2R,3R)-1-((E)-3-methoxy-3-oxoprop-1-en-1-yl)-2-(2-(methylthio)ethyl)-4-oxoazetidine-3-carboxylate (2v). According to the general procedure, $\beta$-lactam $2 \mathbf{v}$ was obtained from 3-(methylthio)propanal oxime (35.7 $\mathrm{mg}, 0.3$ $\mathrm{mmol}$ ) and methyl propiolate $(76.5 \mathrm{mg}, 0.9 \mathrm{mmol})$ as light yellow oil $(43.0 \mathrm{mg}, 50 \%$ yield, 99/1 dr; eluent: PE/EtOAc $=6: 1$ to $2: 1) .{ }^{1} \mathrm{H}$ NMR $\left(400 \mathrm{MHz}, \mathrm{CDCl}_{3}\right) \delta: 7.58-7.54(\mathrm{~d}, J=14.3 \mathrm{~Hz}, 1 \mathrm{H}), 5.58-5.54(\mathrm{~d}, J=14.3 \mathrm{~Hz}, 1 \mathrm{H}), 4.36-4.32$ (m, 1H), 3.96-9.95 (d, $J=3.0 \mathrm{~Hz}, 1 \mathrm{H}), 3.93-3.88(\mathrm{~m}, 1 \mathrm{H}), 3.81(\mathrm{~s}, 3 \mathrm{H}), 3.74(\mathrm{~s}, 3 \mathrm{H}), 2.60-2.57(\mathrm{~m}, 2 \mathrm{H}), 2.42-2.36(\mathrm{~m}$, $1 \mathrm{H}), 2.14(\mathrm{~s}, 3 \mathrm{H}), 1.96-1.89(\mathrm{~m}, 1 \mathrm{H}) \mathrm{ppm} ;{ }^{13} \mathrm{C} \mathrm{NMR}\left(100 \mathrm{MHz}, \mathrm{CDCl}_{3}\right) \delta: 166.9,166.0,159.3,134.1,102.5,60.4$, 55.8, 53.0, 51.6, 30.3, 29.6, 15.8 ppm; IR (Neat): 2953, 1784, 1734, 1632, 1437, 1292, 1169, 966, $843 \mathrm{~cm}^{-1}$; HRMS (ESI) $m / z:[\mathrm{M}+\mathrm{H}]^{+}$calcd. for $\mathrm{C}_{12} \mathrm{H}_{18} \mathrm{NO}_{5} \mathrm{~S}: 288.0900$; Found: 288.0901 .<smiles>COC(=O)/C=C/N1C(=O)C(C(C(C)=O)[N+](=O)[O-])[C@H]1C(=O)[O-]</smiles>

( \pm ) Methyl (2S,3R)-2-((S)-1,2-dinitroethyl)-1-((E)-3-methoxy-3-oxoprop-1-en-1-yl)-4-oxoazetidine-3-carboxylate (2w). According to the general procedure, $\beta$-lactam $\mathbf{2 w}$ was obtained from 2,3-dinitropropanal oxime (49.0 mg, 0.3 $\mathrm{mmol}$ ) and methyl propiolate $(76.5 \mathrm{mg}, 0.9 \mathrm{mmol})$ as light yellow oil $(60.3 \mathrm{mg}, 57 \%$ yield, $86 / 14 \mathrm{dr}$; eluent: PE/EtOAc $=8: 1$ to $3: 1) .{ }^{1} \mathrm{H}$ NMR $\left(400 \mathrm{MHz}, \mathrm{CDCl}_{3}\right) \delta: 7.63-7.59(\mathrm{~d}, J=14.3 \mathrm{~Hz}, 1 \mathrm{H}), 5.91-5.82(\mathrm{~m}, 1 \mathrm{H}), 5.59-5.54(\mathrm{~d}, J=17.1$ 
$\mathrm{Hz}, 1 \mathrm{H}), 5.50-5.44(\mathrm{~m}, 2 \mathrm{H}), 4.68-4.66(\mathrm{~m}, 1 \mathrm{H}), 3.93-9.92(\mathrm{~d}, J=3.1 \mathrm{~Hz}, 1 \mathrm{H}), 3.82(\mathrm{~s}, 3 \mathrm{H}), 3.73(\mathrm{~s}, 3 \mathrm{H}) \mathrm{ppm} ;{ }^{13} \mathrm{C}$ NMR (100 MHz, $\left.\mathrm{CDCl}_{3}\right) \delta$ : 167.0, 165.8, 159.1, 134.0, 132.3, 122.1, 102.6, 61.6, 57.8, 53.1, 51.6 ppm; IR (Neat): 2954, 2851, 1783, 1712, 1631, 1437, 1280, 1166, 965, 842, $766 \mathrm{~cm}^{-1}$; HRMS (ESI) $\mathrm{m} / \mathrm{z}:[\mathrm{M}+\mathrm{Na}]^{+}$calcd. for $\mathrm{C}_{11} \mathrm{H}_{13} \mathrm{~N}_{3} \mathrm{NaO}_{9}$ : 354.0544; Found: 354.0539 .

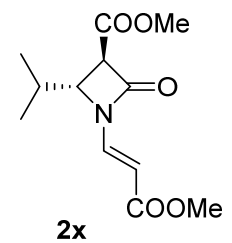

$( \pm) \quad$ Methyl (2R,3R)-2-isopropyl-1-((E)-3-methoxy-3-oxoprop-1-en-1-yl)-4-oxoazetidine-3-carboxylate $(2 x)$. According to the general procedure, $\beta$-lactam $2 \mathbf{x}$ was obtained from sobutyraldehyde oxime $(26.1 \mathrm{mg}, 0.3 \mathrm{mmol})$ and methyl propiolate $(76.5 \mathrm{mg}, 0.9 \mathrm{mmol})$ as light yellow oil $(60.0 \mathrm{mg}, 78 \%$ yield, $95 / 5 \mathrm{dr}$; eluent: $\mathrm{PE} / \mathrm{EtOAc}=6: 1$ to 4:1). ${ }^{1} \mathrm{H}$ NMR (400 MHz, $\left.\mathrm{CDCl}_{3}\right) \delta: 7.50-7.47(\mathrm{~d}, J=14.3 \mathrm{~Hz}, 1 \mathrm{H}), 5.49-5.45(\mathrm{~d}, J=14.3 \mathrm{~Hz}, 1 \mathrm{H}), 4.18-4.14(\mathrm{~m}, 1 \mathrm{H})$, 3.77-3.76 (d, $J=3.0 \mathrm{~Hz}, 1 \mathrm{H}), 3.73(\mathrm{~s}, 3 \mathrm{H}), 3.67(\mathrm{~s}, 3 \mathrm{H}), 2.04-1.96(\mathrm{~m}, 1 \mathrm{H}), 1.58-1.48(\mathrm{~m}, 1 \mathrm{H}), 1.40-1.30(\mathrm{~m}, 2 \mathrm{H})$, 0.94-0.91 (d, $J=7.3 \mathrm{~Hz}, 3 \mathrm{H}) \mathrm{ppm} ;{ }^{13} \mathrm{C}$ NMR $\left(100 \mathrm{MHz}, \mathrm{CDCl}_{3}\right) \delta: 167.0,166.3,159.5,134.1,102.1,60.0,56.5,52.9$, 51.5, 32.9, 18.1, 13.6 ppm; IR (Neat): 2957, 1778, 1733, 1627, 1438, 1293, 1158, 969, 839, 732, $586 \mathrm{~cm}^{-1}$; HRMS (ESI) $m / z:[\mathrm{M}+\mathrm{H}]^{+}$calcd. for $\mathrm{C}_{12} \mathrm{H}_{18} \mathrm{NO}_{5}: 256.1179$; Found: 256.1179 .

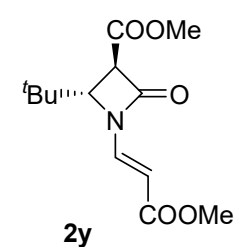

( \pm ) Methyl (2S,3R)-2-(tert-butyl)-1-((E)-3-methoxy-3-oxoprop-1-en-1-yl)-4-oxoazetidine-3-carboxylate (2y). According to the general procedure, $\beta$-lactam $2 \mathbf{y}$ was obtained from pivalaldehyde oxime $(30.3 \mathrm{mg}, 0.3 \mathrm{mmol})$ and methyl propiolate $(76.5 \mathrm{mg}, 0.9 \mathrm{mmol})$ as light yellow oil $(59.4 \mathrm{mg}, 74 \%$ yield, $99 / 1 \mathrm{dr}$; eluent: $\mathrm{PE} / \mathrm{EtOAc}=8: 1$ to 4:1). ${ }^{1} \mathrm{H}$ NMR (400 MHz, $\left.\mathrm{CDCl}_{3}\right) \delta: 7.22-7.18(\mathrm{~d}, J=14.3 \mathrm{~Hz}, 1 \mathrm{H}), 6.10-6.06(\mathrm{~d}, J=14.3 \mathrm{~Hz}, 1 \mathrm{H}), 3.95-3.94(\mathrm{~d}, J=$ $3.1 \mathrm{~Hz}, 1 \mathrm{H}), 3.77(\mathrm{~d}, J=3.1 \mathrm{~Hz}, 1 \mathrm{H}), 3.73(\mathrm{~s}, 3 \mathrm{H}), 3.66(\mathrm{~s}, 3 \mathrm{H}), 0.97(\mathrm{~s}, 9 \mathrm{H}) \mathrm{ppm} ;{ }^{13} \mathrm{C} \mathrm{NMR}\left(100 \mathrm{MHz}, \mathrm{CDCl}_{3}\right) \delta$ : 167.5, 166.6, 160.7, 136.5, 105.7, 66.3, 56.3, 53.0, 51.5, 33.3, 25.8 ppm; IR (Neat): 2957, 1779, 1715, 1624, 1437, $1331,1239,1166 \mathrm{~cm}^{-1}$; HRMS (ESI) $m / z:[\mathrm{M}+\mathrm{H}]^{+}$calcd. for $\mathrm{C}_{13} \mathrm{H}_{20} \mathrm{NO}_{5}: 270.1336$; Found: 270.1332.

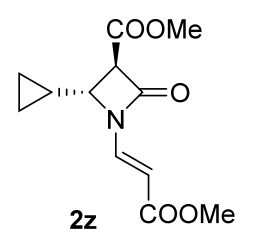

( \pm ) Methyl (2R,3R)-2-cyclopropyl-1-((E)-3-methoxy-3-oxoprop-1-en-1-yl)-4-oxoazetidine-3-carboxylate (2z). According to the general procedure, $\beta$-lactam $2 \mathbf{z}$ was obtained from cyclopropanecarbaldehyde oxime $(25.7 \mathrm{mg}, 0.3$ $\mathrm{mmol}$ ) and methyl propiolate $(76.5 \mathrm{mg}, 0.9 \mathrm{mmol})$ as light yellow oil $(57.7 \mathrm{mg}, 76 \%$ yield, $87 / 13 \mathrm{dr}$; eluent: PE/EtOAc $=8: 1$ to $4: 1) .{ }^{1} \mathrm{H}$ NMR $\left(400 \mathrm{MHz}, \mathrm{CDCl}_{3}\right) \delta: 7.62-7.58(\mathrm{~d}, J=14.3 \mathrm{~Hz}, 1 \mathrm{H}), 5.70-5.66(\mathrm{~d}, J=14.3 \mathrm{~Hz}, 1 \mathrm{H}), 3.99-3.98$ (d, $J=3.1 \mathrm{~Hz}, 1 \mathrm{H}), 3.80$ (s, 3H), 3.75 (s, 3H), 3.62-3.59 (m, 1H), 1.08-0.99 (m, 1H), 0.92-0.87 (m, 1H), 0.74-0.67 (m, $1 \mathrm{H}), 0.59-0.53(\mathrm{~m}, 1 \mathrm{H}), 0.44-0.38(\mathrm{~m}, 1 \mathrm{H}) \mathrm{ppm} ;{ }^{13} \mathrm{C} \mathrm{NMR}\left(100 \mathrm{MHz}, \mathrm{CDCl}_{3}\right) \delta: 167.2,166.1,159.7,134.2,103.1$, 61.7, 60.5, 52.9, 51.5, 12.0, 6.3, 1.7 ppm; IR (Neat): 3005, 2953, 1778, 1707, 1628, 1437, 1280, 1159, 961, 838, 731, $573 \mathrm{~cm}^{-1}$;HRMS (ESI) $m / z$ : $[\mathrm{M}+\mathrm{H}]^{+}$calcd. for $\mathrm{C}_{12} \mathrm{H}_{16} \mathrm{NO}_{5}$ : 254.1023; Found: 254.1027. 


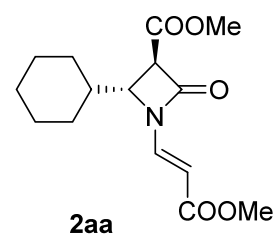

( \pm ) Methyl (2R,3R)-2-cyclohexyl-1-((E)-3-methoxy-3-oxoprop-1-en-1-yl)-4-oxoazetidine-3-carboxylate (2aa). According to the general procedure, $\beta$-lactam 2aa was obtained from cyclohexanecarbaldehyde oxime $(38.1 \mathrm{mg}, 0.3$ $\mathrm{mmol}$ ) and methyl propiolate $(76.5 \mathrm{mg}, 0.9 \mathrm{mmol})$ as light yellow oil $(65.5 \mathrm{mg}, 74 \%$ yield, $91 / 9 \mathrm{dr}$; eluent: PE/EtOAc $=8: 1$ to $4: 1) .{ }^{1} \mathrm{H}$ NMR $\left(400 \mathrm{MHz}, \mathrm{CDCl}_{3}\right) \delta: 7.56-7.52(\mathrm{~d}, J=14.3 \mathrm{~Hz}, 1 \mathrm{H}), 5.67-5.63(\mathrm{~d}, J=14.3 \mathrm{~Hz}, 1 \mathrm{H}), 4.16-4.14$ (m, 1H), 3.94-3.93 (d, $J=3.2 \mathrm{~Hz}, 1 \mathrm{H}), 3.79$ (s, 3H), 3.74 (s, 3H), 1.98-1.90 (m, 1H), 1.83-1.79 (m, 4H), 1.75-1.72 (d, $J$ $=11.9 \mathrm{~Hz}, 1 \mathrm{H}), 1.62-1.13(\mathrm{~m}, 3 \mathrm{H}), 1.08-0.94(\mathrm{~m}, 2 \mathrm{H}) \mathrm{ppm} ;{ }^{13} \mathrm{C} \mathrm{NMR}\left(100 \mathrm{MHz}, \mathrm{CDCl}_{3}\right) \delta: 167.2,166.6,159.9,135.0$, 102.6, 61.2, 56.4, 52.9, 51.5, 37.8, 29.1, 25.9, 25.8, 25.6, 25.3 ppm; IR (Neat): 2928, 2854, 1778, 1711, 1627, 1438, 1248, 1159, 969, 842, 730, 647, $586 \mathrm{~cm}^{-1}$; HRMS (ESI) $\mathrm{m} / z$ : $[\mathrm{M}+\mathrm{H}]^{+}$calcd. for $\mathrm{C}_{15} \mathrm{H}_{22} \mathrm{NO}_{5}$ : 296.1492; Found: 296.1494.

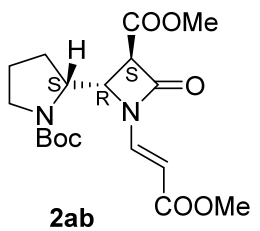

tert-Butyl

(S)-2-((2R,3S)-1-((E)-3-methoxy-3-oxoprop-1-en-1-yl)-3-(methoxycarbonyl)-4-oxoazetidin-2-

yl)pyrrolidine-1 carboxylate (2ab). According to the general procedure, $\beta$-lactam 2ab was obtained from tert-butyl $(R)$-2-((hydroxyimino)methyl)pyrrolidine-1-carboxylate $(64.2 \mathrm{mg}, 0.3 \mathrm{mmol})$ and methyl propiolate $(76.5 \mathrm{mg}, 0.9$ mmol) as light yellow solid $(80.2 \mathrm{mg}, 70 \%$ yield, 99/1 dr; eluent: PE/EtOAc $=4: 1$ to 2:1). The enantiomeric excess was determined by chiral HPLC analysis using a Chiralpak AD-H column, ee $=95 \%$ ( $n$-hexane/ethanol $=95 / 5$, flow rate $=1 \mathrm{~mL} / \mathrm{min}, \lambda=254 \mathrm{~nm}, \mathrm{~T}=20^{\circ} \mathrm{C}, \mathrm{t}_{\mathrm{r}}($ minor $)=33.427 \mathrm{~min}, \mathrm{t}_{\mathrm{r}}$ (major $\left.)=48.340 \mathrm{~min}\right) .[\alpha]_{\mathrm{D}}{ }^{25}=-19.2(\mathrm{c}=1.0 \mathrm{in}$ $\left.\mathrm{CHCl}_{3}\right) .{ }^{1} \mathrm{H}$ NMR $\left(400 \mathrm{MHz}, \mathrm{CDCl}_{3}\right) \delta: 7.51-7.48(\mathrm{~d}, J=14.3 \mathrm{~Hz}, 1 \mathrm{H}), 5.66-5.62(\mathrm{~d}, J=14.3 \mathrm{~Hz}, 1 \mathrm{H}), 4.77(\mathrm{~s}, 1 \mathrm{H})$, 4.22-4.20 (m, 1H), 3.90-3.89 (d, $J=3.1 \mathrm{~Hz}, 1 \mathrm{H}), 3.79$ (s, 3H), 3.73 (s, 3H), 3.49 (s, 1H), 3.26-3.22 (m, 1H), 2.07-2.03 (m, 1H), 1.87-1.77 (m, 3H), 1.49 (s, 9H) ppm; $\left.{ }^{13} \mathrm{C} \mathrm{NMR} \mathrm{(100} \mathrm{MHz,} \mathrm{CDCl}_{3}\right) \delta: 167.3,165.9,159.9,154.6,135.1,103.3$, 80.6, 57.6, 57.4, 53.0, 51.6, 47.0, 28.3 ppm; IR (Neat): 2977, 2954, 2253, 1784, 1738, 1632, 1477, 1437, 1285, 1159, 971, 911, 846, 727, $647 \mathrm{~cm}^{-1}$; HRMS (ESI) $\mathrm{m} / z$ : $[\mathrm{M}+\mathrm{H}]^{+}$calcd. for $\mathrm{C}_{18} \mathrm{H}_{27} \mathrm{~N}_{2} \mathrm{O}_{7}: 383.1813$; Found: 383.1808 .

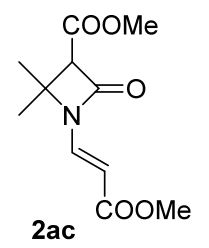

Methyl (E)-1-(3-methoxy-3-oxoprop-1-en-1-yl)-2,2-dimethyl-4-oxoazetidine-3-carboxylate (2ac). According to the general procedure, $\beta$-lactam 2 ac was obtained from propan-2-one oxime $(22.1 \mathrm{mg}, 0.3 \mathrm{mmol})$ and methyl propiolate (76.5 mg, $0.9 \mathrm{mmol})$ as light yellow oil $(56.8 \mathrm{mg}, 79 \%$ yield; eluent: $\mathrm{PE} / \mathrm{EtOAc}=6: 1$ to $3: 1) .{ }^{1} \mathrm{H} \mathrm{NMR}(400 \mathrm{MHz}$, $\left.\mathrm{CDCl}_{3}\right) \delta: 7.44-7.40(\mathrm{~d}, J=14.6 \mathrm{~Hz}, 1 \mathrm{H}), 5.68-5.64(\mathrm{~d}, J=14.5 \mathrm{~Hz}, 1 \mathrm{H}), 3.93(\mathrm{~s}, 1 \mathrm{H}), 3.79(\mathrm{~s}, 3 \mathrm{H}), 3.74(\mathrm{~s}, 3 \mathrm{H}), 1.66$ (s, 3H), 1.54 (s, 3H) ppm; ${ }^{13} \mathrm{C}$ NMR $\left(100 \mathrm{MHz}, \mathrm{CDCl}_{3}\right) \delta: 167.3,166.0,159.5,132.7,102.6,65.1,61.1,52.6,51.6$, 
25.8, 20.9 ppm; IR (Neat): 2953, 1777, 1732, 1628, 1437, 1239, 1155, 961, 843, 746, $569 \mathrm{~cm}^{-1}$; HRMS (ESI) $m / z$ : $[\mathrm{M}+\mathrm{H}]^{+}$calcd. for $\mathrm{C}_{11} \mathrm{H}_{16} \mathrm{NO}_{5}$ : 242.1023; Found: 242.1020 .

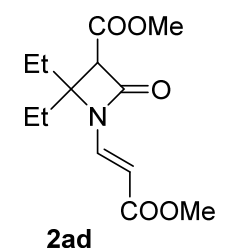

Methyl (E)-2,2-diethyl-1-(3-methoxy-3-oxoprop-1-en-1-yl)-4-oxoazetidine-3-carboxylate (2ad). According to the general procedure, $\beta$-lactam 2 ad was obtained from pentan-3-one oxime $(30.3 \mathrm{mg}, 0.3 \mathrm{mmol})$ and methyl propiolate (76.5 mg, $0.9 \mathrm{mmol}$ ) as light yellow oil $\left(61.6 \mathrm{mg}, 76 \%\right.$ yield; eluent: PE/EtOAc $=6: 1$ to 3:1). ${ }^{1} \mathrm{H}$ NMR (400 MHz, $\left.\mathrm{CDCl}_{3}\right) \delta: 7.43-7.39(\mathrm{~d}, J=14.4 \mathrm{~Hz}, 1 \mathrm{H}), 5.78-5.75(\mathrm{~d}, J=14.4 \mathrm{~Hz}, 1 \mathrm{H}), 3.95(\mathrm{~s}, 1 \mathrm{H}), 3.78(\mathrm{~s}, 3 \mathrm{H}), 3.73(\mathrm{~s}, 3 \mathrm{H}), 2.10-$ $2.00(\mathrm{~m}, 2 \mathrm{H}), 1.96-1.91(\mathrm{~m}, 1 \mathrm{H}), 1.86-1.80(\mathrm{~m}, 1 \mathrm{H}), 1.02-0.98(\mathrm{~d}, J=7.4 \mathrm{~Hz}, 3 \mathrm{H}), 0.92-0.88(\mathrm{~d}, J=7.5 \mathrm{~Hz}, 3 \mathrm{H}) \mathrm{ppm}$; ${ }^{13} \mathrm{C}$ NMR $\left(100 \mathrm{MHz}, \mathrm{CDCl}_{3}\right) \delta: 167.4,166.4,160.3,133.8,101.1,68.0,60.5,52.7,51.5,28.7,25.9,8.2,7.9 \mathrm{ppm}$; IR (Neat): 2951, 1777, 1711, 1624, 1437, 1287, 1157, 964, 846, 736, 611, $474 \mathrm{~cm}^{-1}$; HRMS (ESI) $\mathrm{m} / z$ : $[\mathrm{M}+\mathrm{H}]^{+}$calcd. for $\mathrm{C}_{13} \mathrm{H}_{20} \mathrm{NO}_{5}$ : 270.1336; Found: 270.1335 .

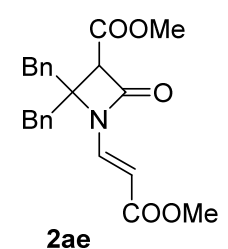

Methyl (E)-2,2-dibenzyl-1-(3-methoxy-3-oxoprop-1-en-1-yl)-4-oxoazetidine-3-carboxylate (2ae). According to the general procedure, $\beta$-lactam 2ae was obtained from 1,3-diphenylpropan-2-one oxime ( $67.5 \mathrm{mg}, 0.3 \mathrm{mmol}$ ) and methyl propiolate $(76.5 \mathrm{mg}, 0.9 \mathrm{mmol})$ as light yellow oil $(81.6 \mathrm{mg}, 69 \%$ yield; eluent: $\mathrm{PE} / \mathrm{EtOAc}=8: 1$ to $3: 1) .{ }^{1} \mathrm{H}$ NMR $(400$ $\left.\mathrm{MHz} \mathrm{CDCl}_{3}\right) \delta$ : 7.25-7.16 (m, 11H), $6.21(\mathrm{~s}, 1 \mathrm{H}), 4.47-4.43(\mathrm{~m}, 1 \mathrm{H}), 4.00(\mathrm{~s}, 2 \mathrm{H}), 3.69(\mathrm{~s}, 3 \mathrm{H}), 3.59(\mathrm{~s}, 3 \mathrm{H}), 2.97-2.92$ $(\mathrm{m}, 1 \mathrm{H}), 2.32-2.26(\mathrm{~m}, 1 \mathrm{H}) \mathrm{ppm} ;{ }^{13} \mathrm{C}$ NMR $\left(100 \mathrm{MHz}, \mathrm{CDCl}_{3}\right) \delta: 169.6,167.0,160.5,137.8,134.4,134.3,129.3$, 129.0, 128.6, 128.4, 127.7, 127.1, 119.6, 59.5, 52.7, 51.8, 51.3, 40.2, 34.6 ppm; IR (Neat): 3026, 2953, 2852, 1763 , 1732, 1436, 1387, 1207, 1160, 1075, 922, 753, $701 \mathrm{~cm}^{-1}$; HRMS (ESI) $\mathrm{m} / \mathrm{z}:[\mathrm{M}+\mathrm{H}]^{+}$calcd. for $\mathrm{C}_{23} \mathrm{H}_{24} \mathrm{NO}_{5}: 394.1649$; Found: 394.1654.

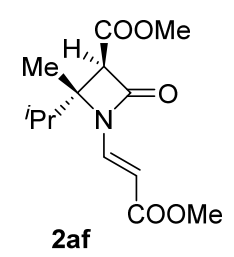

( \pm ) Methyl (2R,3R)-2-isopropyl-1-((E)-3-methoxy-3-oxoprop-1-en-1-yl)-2-methyl-4-oxoazetidine-3-carboxylate (2af). According to the general procedure, $\beta$-lactam 2af was obtained from 3-methylbutan-2-one oxime (30.3 mg, 0.3 $\mathrm{mmol}$ ) and methyl propiolate $(76.5 \mathrm{mg}, 0.9 \mathrm{mmol})$ as light yellow oil $(58.1 \mathrm{mg}, 72 \%$ yield; eluent: $\mathrm{PE} / \mathrm{EtOAc}=8: 1$ to 3:1). ${ }^{1} \mathrm{H}$ NMR (400 MHz, $\left.\mathrm{CDCl}_{3}\right) \delta: 7.45-7.41(\mathrm{~d}, J=14.5 \mathrm{~Hz}, 1 \mathrm{H}), 5.71-5.67(\mathrm{~d}, J=14.5 \mathrm{~Hz}, 1 \mathrm{H}), 3.98(\mathrm{~s}, 1 \mathrm{H}), 3.78$ (s, 3H), 3.74 (s, 3H), 2.25-2.18 (m, 1H), 1.52 (s, 3H), 1.08-1.06 (d, $J=6.7 \mathrm{~Hz}, 3 \mathrm{H}), 0.95-0.93(\mathrm{~d}, J=6.8 \mathrm{~Hz}, 3 \mathrm{H}) \mathrm{ppm}$; ${ }^{13} \mathrm{C}$ NMR $\left(100 \mathrm{MHz}, \mathrm{CDCl}_{3}\right) \delta: 167.3,166.5,160.1,133.3,102.8,67.5,59.0,52.5,51.5,34.5,18.1,17.8,16.5 \mathrm{ppm}$; IR (Neat): 2955, 1778, 1734, 1632, 1437, 1385, 1248, 1158, 971, 845, 770, $586 \mathrm{~cm}^{-1}$; HRMS (ESI) $\mathrm{m} / z$ : [M+H] $]^{+}$calcd. for $\mathrm{C}_{13} \mathrm{H}_{20} \mathrm{NO}_{5}$ : 270.1336; Found: 270.1331. 


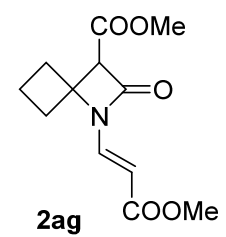

Methyl (E)-1-(3-methoxy-3-oxoprop-1-en-1-yl)-2-oxo-1-azaspiro[3.3] heptane-3-carboxylate (2ag). According to the general procedure, $\beta$-lactam 2ag was obtained from cyclobutanone oxime $(25.5 \mathrm{mg}, 0.3 \mathrm{mmol})$ and methyl propiolate $(76.5 \mathrm{mg}, 0.9 \mathrm{mmol})$ as light yellow oil $(56.4 \mathrm{mg}, 74 \%$ yield; eluent: $\mathrm{PE} / \mathrm{EtOAc}=6: 1$ to $3: 1) .{ }^{1} \mathrm{H} \mathrm{NMR}(400$ $\left.\mathrm{MHz}, \mathrm{CDCl}_{3}\right) \delta: 7.46-7.42(\mathrm{dd}, J=14.6,0.6 \mathrm{~Hz}, 1 \mathrm{H}), 5.93-5.89(\mathrm{~d}, J=14.5 \mathrm{~Hz}, 1 \mathrm{H}), 4.08(\mathrm{~s}, 1 \mathrm{H}), 3.84(\mathrm{~d}, J=0.5 \mathrm{~Hz}$, $3 \mathrm{H}), 3.76(\mathrm{~d}, J=0.6 \mathrm{~Hz}, 3 \mathrm{H}), 2.82-2.74(\mathrm{~m}, 1 \mathrm{H}), 2.71-2.63(\mathrm{~m}, 1 \mathrm{H}), 2.42-2.34(\mathrm{~m}, 1 \mathrm{H}), 2.33-2.25(\mathrm{~m}, 1 \mathrm{H}), 2.00-1.85$ (m, 2H) ppm; ${ }^{13} \mathrm{C}$ NMR (100 MHz, $\left.\mathrm{CDCl}_{3}\right) \delta: 167.3,166.5,159.3,132.9,103.2,64.9,63.8,52.6,51.6,31.5,27.9,13.3$ ppm; IR (Neat): 2953, 1777, 1708, 1625, 1437, 1290, 1152, 965, 844, 732, 671, $594 \mathrm{~cm}^{-1}$; HRMS (ESI) $m / z:[M+H]^{+}$ calcd. for $\mathrm{C}_{12} \mathrm{H}_{16} \mathrm{NO}_{5}$ : 254.1023; Found: 254.1022.

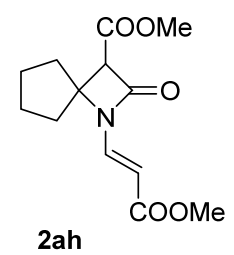

Methyl (E)-1-(3-methoxy-3-oxoprop-1-en-1-yl)-2-oxo-1-azaspiro[3.4]octane-3-carboxylate (2ah). According to the general procedure, $\beta$-lactam 2ah was obtained from cyclopentanone oxime (30.1 $\mathrm{mg}, 0.3 \mathrm{mmol})$ and methyl propiolate (76.5 mg, $0.9 \mathrm{mmol})$ as light yellow oil $\left(55.5 \mathrm{mg}, 61 \%\right.$ yield; eluent: PE/EtOAc $=6: 1$ to 3:1). ${ }^{1} \mathrm{H}$ NMR (400 $\left.\mathrm{MHz}, \mathrm{CDCl}_{3}\right) \delta: 7.31-7.27$ (d, $\left.J=14.5 \mathrm{~Hz}, 1 \mathrm{H}\right), 5.68-5.64(\mathrm{~d}, J=14.5 \mathrm{~Hz}, 1 \mathrm{H}), 3.93(\mathrm{~s}, 1 \mathrm{H}), 3.72(\mathrm{~s}, 3 \mathrm{H}), 3.67(\mathrm{~s}, 3 \mathrm{H})$, 2.20-2.13 (m, 1H), 2.04-1.88 (m, 2H), 1.86-1.74 (m, 3H), 1.72-1.64 (m, 2H) ppm; ${ }^{13} \mathrm{C}$ NMR $\left(100 \mathrm{MHz}, \mathrm{CDCl}_{3}\right) \delta$ : 167.4, 166.5, 159.9, 132.8, 103.3, 70.5, 66.1, 52.6, 51.6, 35.7, 30.1, 24.2, 23.9 ppm; IR (Neat): 2954, 2875, 1781, 1714, 1628, 1438, 1251, 1164, 1046, $969 \mathrm{~cm}^{-1}$; HRMS (ESI) $\mathrm{m} / \mathrm{z}:[\mathrm{M}+\mathrm{H}]^{+}$calcd. for $\mathrm{C}_{13} \mathrm{H}_{18} \mathrm{NO}_{5}$ : 268.1179; Found: 268.1171.

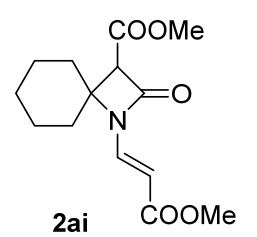

Methyl (E)-1-(3-methoxy-3-oxoprop-1-en-1-yl)-2-oxo-1-azaspiro[3.5]nonane-3-carboxylate (2ai). According to the general procedure, $\beta$-lactam 2ai was obtained from cyclohexanone oxime $(34.0 \mathrm{mg}, 0.3 \mathrm{mmol})$ and methyl propiolate $(76.5 \mathrm{mg}, 0.9 \mathrm{mmol})$ as light yellow oil $(46.7 \mathrm{mg}, 55 \%$ yield; eluent: $\mathrm{PE} / \mathrm{EtOAc}=6: 1$ to $3: 1) .{ }^{1} \mathrm{H}$ NMR $(400$ $\left.\mathrm{MHz}, \mathrm{CDCl}_{3}\right) \delta: 7.24-7.20(\mathrm{~d}, J=13.9 \mathrm{~Hz}, 1 \mathrm{H}), 5.80-5.77(\mathrm{~d}, J=14.5 \mathrm{~Hz}, 1 \mathrm{H}), 3.76(\mathrm{~s}, 1 \mathrm{H}), 3.71(\mathrm{~s}, 3 \mathrm{H}), 3.67(\mathrm{~s}, 3 \mathrm{H})$, 2.12-2.08 (m, 1H), 1.97-1.90 (m, 1H), 1.86-1.76 (m, 2H), 1.75-1.65 (m, 3H), 1.43-1.33 (m, 1H), 1.20-1.07 (m, 2H) ppm; ${ }^{13} \mathrm{C}$ NMR (100 MHz, $\left.\mathrm{CDCl}_{3}\right) \delta: 167.5,166.6,160.2,132.8,103.8,65.1,64.1,52.6,51.5,36.2,29.8,24.5,23.6$, $23.1 \mathrm{ppm}$; IR (Neat): 2951, 1778, 1708, 1628, 1496, 1242, 1161, 970, 842, 755, 692, $512 \mathrm{~cm}^{-1}$; HRMS (ESI) $\mathrm{m} / \mathrm{z}$ : $[\mathrm{M}+\mathrm{H}]^{+}$calcd. for $\mathrm{C}_{14} \mathrm{H}_{20} \mathrm{NO}_{5}$ : 282.1336; Found: 282.1330 .

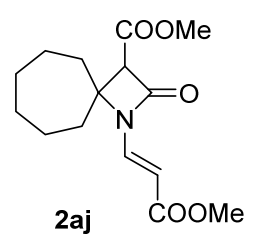


Methyl (E)-1-(3-methoxy-3-oxoprop-1-en-1-yl)-2-oxo-1-azaspiro[3.6]decane-3-carboxylate (2aj). According to the general procedure, $\beta$-lactam 2aj was obtained from cycloheptanone oxime $(38.1 \mathrm{mg}, 0.3 \mathrm{mmol})$ and methyl propiolate (76.5 mg, $0.9 \mathrm{mmol})$ as light yellow oil $(62.0 \mathrm{mg}, 52 \%$ yield; eluent: PE/EtOAc $=6: 1$ to $3: 1) .{ }^{1} \mathrm{H}$ NMR (400 MHz, $\left.\mathrm{CDCl}_{3}\right) \delta: 7.28-7.24(\mathrm{~d}, J=14.5 \mathrm{~Hz}, 1 \mathrm{H}), 5.80-5.77$ (d, $\left.J=14.5 \mathrm{~Hz}, 1 \mathrm{H}\right), 3.83$ (s, 1H), $3.72(\mathrm{~s}, 3 \mathrm{H}), 3.67$ (s, 3H), 2.19$2.07(\mathrm{~m}, 2 \mathrm{H}), 1.98-1.92(\mathrm{~m}, 1 \mathrm{H}), 1.88-1.82(\mathrm{~m}, 1 \mathrm{H}), 1.77-1.70(\mathrm{~m}, 1 \mathrm{H}), 1.60-1.57(\mathrm{~m}, 6 \mathrm{H}), 1.38-1.29(\mathrm{~m}, 1 \mathrm{H}) \mathrm{ppm}$; ${ }^{13} \mathrm{C}$ NMR (100 MHz, $\left.\mathrm{CDCl}_{3}\right) \delta: 170.0,167.1,158.1,139.5,115.9,58.7,52.7,52.0,50.6,35.5,31.6,29.2,26.8,26.2$, 25.6 ppm; IR (Neat): 2951, 1776, 1711, 1623, 1437, 1247, 1154, 965, 845, $736 \mathrm{~cm}^{-1}$; HRMS (ESI) $\mathrm{m} / z$ : [M+H] $]^{+}$calcd. for $\mathrm{C}_{15} \mathrm{H}_{22} \mathrm{NO}_{5}$ : 296.1492; Found: 296.1497.

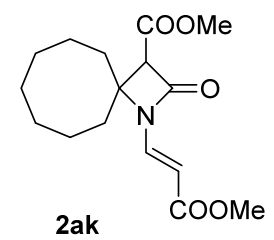

Methyl (E)-1-(3-methoxy-3-oxoprop-1-en-1-yl)-2-oxo-1-azaspiro[3.7]undecane-3-carboxylate (2ak). According to the general procedure, $\beta$-lactam 2ak was obtained from cyclooctanone oxime $(42.3 \mathrm{mg}, 0.3 \mathrm{mmol})$ and methyl propiolate $(76.5 \mathrm{mg}, 0.9 \mathrm{mmol})$ as light yellow oil $(46.8 \mathrm{mg}, 51 \%$ yield; eluent: $\mathrm{PE} / \mathrm{EtOAc}=6: 1$ to $3: 1) .{ }^{1} \mathrm{H} \mathrm{NMR}(400$ $\left.\mathrm{MHz}, \mathrm{CDCl}_{3}\right) \delta$ : 7.34-7.31 (d, $\left.J=14.4 \mathrm{~Hz}, 1 \mathrm{H}\right), 5.87-5.84(\mathrm{~d}, J=14.4 \mathrm{~Hz}, 1 \mathrm{H}), 3.90$ (s, 1H), 3.79 (s, 3H), 3.74 (s, 3H), 3.07-2.80 (m, 1H), 2.26-2.15 (m, 2H), 2.05-1.97 (m, 1H), 1.95-1.89 (m, 1H), 1.84-1.79 (m, 1H), 1.70-1.56 (m, 7H), 1.45-1.37 (m, 1H) ppm; ${ }^{13} \mathrm{C}$ NMR (100 MHz, $\left.\mathrm{CDCl}_{3}\right) \delta: 170.0,167.2,157.8,136.4,112.2,58.9,52.7,52.1,50.3,35.4$, 30.3, 28.0, 26.5, 25.7, 25.3, 25.0 ppm; IR (Neat): 2924, 2852, 1762, 1735, 1437, 1338, 1323, 1209, $1165 \mathrm{~cm}^{-1}$; HRMS (ESI) $m / z:[\mathrm{M}+\mathrm{H}]^{+}$calcd. for $\mathrm{C}_{16} \mathrm{H}_{24} \mathrm{NO}_{5}: 310.1649$; Found: 310.1653 .

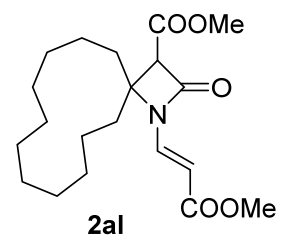

Methyl (E)-1-(3-methoxy-3-oxoprop-1-en-1-yl)-2-oxo-1-azaspiro[3.9]tridecane-3-carboxylate (2al). According to the general procedure, $\beta$-lactam 2al was obtained from cyclododecanone oxime $(59.1 \mathrm{mg}, 0.3 \mathrm{mmol})$ and methyl propiolate (76.5 mg, $0.9 \mathrm{mmol})$ as light yellow oil $\left(48.5 \mathrm{mg}, 44 \%\right.$ yield; eluent: PE/EtOAc $=8: 1$ to 4:1). ${ }^{1} \mathrm{H}$ NMR (400 $\left.\mathrm{MHz}, \mathrm{CDCl}_{3}\right) \delta: 7.18-7.14(\mathrm{~d}, J=14.3 \mathrm{~Hz}, 1 \mathrm{H}), 6.21-6.18(\mathrm{~d}, J=14.3 \mathrm{~Hz}, 1 \mathrm{H}), 3.89$ (s, 1H), $3.78(\mathrm{~s}, 3 \mathrm{H}), 3.74(\mathrm{~s}, 3 \mathrm{H})$, 2.24-2.13 (m, 1H), 1.94-1.88 (m, 1H), 1.77-1.67 (m, 1H), 1.46-1.25 (m, 17H), 0.94-0.88 (m, 2H) ppm; ${ }^{13} \mathrm{C}$ NMR $(100$ $\left.\mathrm{MHz}, \mathrm{CDCl}_{3}\right) \delta: 170.0,167.2,157.8,135.2,115.6,58.3,52.7,52.0,50.1,35.3,27.3,25.2,24.7,24.5,24.2,23.9,23.8$, 23.3, 22.5, 22.1 ppm; IR (Neat): 2931, 2860, 1780, 1715, 1621, 1438, 1248, 1161, 961, $846 \mathrm{~cm}^{-1}$; HRMS (ESI) $\mathrm{m} / \mathrm{z}$ : $[\mathrm{M}+\mathrm{H}]^{+}$calcd. for $\mathrm{C}_{20} \mathrm{H}_{32} \mathrm{NO}_{5}: 366.2275$; Found: 366.2280 .

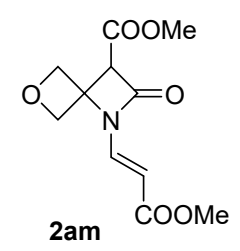

Methyl (E)-1-(3-methoxy-3-oxoprop-1-en-1-yl)-2-oxo-6-oxa-1-azaspiro[3.3]heptane-3-carboxylate

(2am).

According to the general procedure, $\beta$-lactam 2am was obtained from oxetan-3-one oxime $(26.1 \mathrm{mg}, 0.3 \mathrm{mmol})$ and methyl propiolate $(76.5 \mathrm{mg}, 0.9 \mathrm{mmol})$ as light yellow oil $(55.8 \mathrm{mg}, 73 \%$ yield; eluent: $\mathrm{PE} / \mathrm{EtOAc}=6: 1$ to $3: 1) .{ }^{1} \mathrm{H}$ 
NMR (400 MHz, $\left.\mathrm{CDCl}_{3}\right) \delta: 7.60-7.56(\mathrm{~d}, J=14.7 \mathrm{~Hz}, 1 \mathrm{H}), 6.08-6.05(\mathrm{~d}, J=14.6 \mathrm{~Hz}, 1 \mathrm{H}), 5.04-4.94(\mathrm{~m}, 4 \mathrm{H}), 4.09$ (s, $1 \mathrm{H}), 3.75$ (s, 3H), 3.71 (s, 3H) ppm; ${ }^{13} \mathrm{C}$ NMR (100 MHz, $\left.\mathrm{CDCl}_{3}\right) \delta: 167.0,165.3,157.7,131.9,103.5,75.9,74.7,63.6$, 61.6, 53.1, 51.8 ppm; IR (Neat): 2954, 2884, 1787, 1713, 1634, 1439, 1256, 1170, 973, $848 \mathrm{~cm}^{-1}$; HRMS (ESI) $m / z$ : $[\mathrm{M}+\mathrm{H}]^{+}$calcd. for $\mathrm{C}_{11} \mathrm{H}_{14} \mathrm{NO}_{6}$ : 256.0816; Found: 256.0818 .

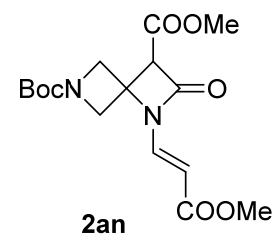

6-(tert-Butyl) 3-methyl $\quad$ (E)-1-(3-methoxy-3-oxoprop-1-en-1-yl)-2-oxo-1,6-diazaspiro[3.3]heptane-3,6dicarboxylate (2an). According to the general procedure, $\beta$-lactam 2an was obtained from tert-butyl 3(hydroxyimino)azetidine-1-carboxylate $(55.8 \mathrm{mg}, 0.3 \mathrm{mmol}$ ) and methyl propiolate $(76.5 \mathrm{mg}, 0.9 \mathrm{mmol})$ as light yellow oil (73.3 mg, 69\% yield; eluent: $\mathrm{PE} / \mathrm{EtOAc}=6: 1$ to $3: 1) .{ }^{1} \mathrm{H} \mathrm{NMR}\left(400 \mathrm{MHz}, \mathrm{CDCl}_{3}\right) \delta: 7.51-7.48(\mathrm{~d}, J=14.7$ Hz, 1H), 5.81-5.78 (d, $J=14.6 \mathrm{~Hz}, 1 \mathrm{H}), 4.29-4.21$ (m, 4H), 4.09 (s, 1H), 3.76 (s, 3H), 3.70 (s, 3H), 1.41 (s, 9H) ppm; ${ }^{13} \mathrm{C}$ NMR $\left(100 \mathrm{MHz}, \mathrm{CDCl}_{3}\right) \delta: 166.8,165.3,157.8,156.0,131.7,103.2,81.1,62.4,58.7,55.8,54.1,53.1,51.8,28.2$ ppm; IR (Neat): 2954, 1792, 1704, 1634, 1390, 1256, 1166, 966, 851, $733 \mathrm{~cm}^{-1}$; HRMS (ESI) $m / z:[\mathrm{M}+\mathrm{H}]^{+}$calcd. for $\mathrm{C}_{16} \mathrm{H}_{23} \mathrm{~N}_{2} \mathrm{O}_{7}$ : 355.1500; Found: 355.1506 .

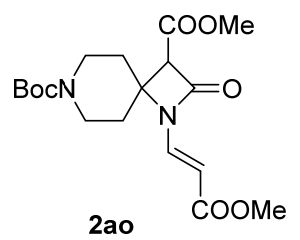

7-(tert-Butyl) 3-methyl (E)-1-(3-methoxy-3-oxoprop-1-en-1-yl)-2-oxo-1,7-diazaspiro[3.5]nonane-3,7dicarboxylate (2ao). According to the general procedure, $\beta$-lactam 2ao was obtained from tert-butyl 4(hydroxyimino)piperidine-1-carboxylate $(64.2 \mathrm{mg}, 0.3 \mathrm{mmol})$ and methyl propiolate $(76.5 \mathrm{mg}, 0.9 \mathrm{mmol})$ as light yellow oil (59.6 mg, 52\% yield; eluent: $\mathrm{PE} / \mathrm{EtOAc}=6: 1$ to 3:1). ${ }^{1} \mathrm{H} \mathrm{NMR}\left(400 \mathrm{MHz}, \mathrm{CDCl}_{3}\right) \delta: 7.25-7.21(\mathrm{~d}, J=14.6$ Hz, 1H), 5.81-5.78 (d, $J=14.5 \mathrm{~Hz}, 1 \mathrm{H}), 4.21-4.08$ (d, $J=54.4 \mathrm{~Hz}, 2 \mathrm{H}), 3.85$ (s, 1H), 3.73 (s, 3H), 3.67 (s, 3H), 2.79$2.60(\mathrm{~d}, J=77.8 \mathrm{~Hz}, 2 \mathrm{H}), 2.16-2.06(\mathrm{~m}, 2 \mathrm{H}), 2.02-1.94(\mathrm{~m}, 1 \mathrm{H}), 1.69-1.66(\mathrm{~d}, J=11.4 \mathrm{~Hz}, 1 \mathrm{H}), 1.41(\mathrm{~s}, 9 \mathrm{H}) \mathrm{ppm} ;{ }^{13} \mathrm{C}$ NMR (100 MHz, $\left.\mathrm{CDCl}_{3}\right) \delta: 167.2,166.2,159.3,154.3,132.4,104.5,80.5,63.5,63.1,52.9,51.7,35.4,29.4,28.4$ ppm; IR (Neat): 2953, 2855, 1782, 1797, 1627, 1433, 1250, 1164, 1077, $1043 \mathrm{~cm}^{-1}$; HRMS (ESI) m/z: [M+H] ${ }^{+}$calcd. for $\mathrm{C}_{18} \mathrm{H}_{27} \mathrm{~N}_{2} \mathrm{O}_{7}$ : 383.1813; Found: 383.1809 .

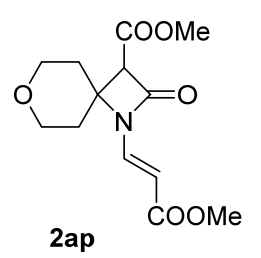

Methyl (E)-1-(3-methoxy-3-oxoprop-1-en-1-yl)-2-oxo-7-oxa-1-azaspiro[3.5]nonane-3-carboxylate

(2ap).

According to the general procedure, $\beta$-lactam 2ap was obtained from tetrahydro- $4 H$-pyran-4-one oxime (34.5 mg, 0.3 $\mathrm{mmol})$ and methyl propiolate $(76.5 \mathrm{mg}, 0.9 \mathrm{mmol})$ as light yellow oil $(30.6 \mathrm{mg}, 36 \%$ yield; eluent: $\mathrm{PE} / \mathrm{EtOAc}=4: 1$ to 2:1). ${ }^{1} \mathrm{H}$ NMR (400 MHz, $\left.\mathrm{CDCl}_{3}\right) \delta: 7.34-7.31(\mathrm{~d}, J=14.5 \mathrm{~Hz}, 1 \mathrm{H}), 5.92-5.88(\mathrm{~d}, J=14.5 \mathrm{~Hz}, 1 \mathrm{H}), 4.13-4.08(\mathrm{~m}, 1 \mathrm{H})$, 4.01-3.97 (m, 1H), $3.95(\mathrm{~s}, 1 \mathrm{H}), 3.80(\mathrm{~s}, 3 \mathrm{H}), 3.74(\mathrm{~s}, 3 \mathrm{H}), 3.56-3.49(\mathrm{~m}, 1 \mathrm{H}), 3.37-3.30(\mathrm{~m}, 1 \mathrm{H}), 2.42-2.34(\mathrm{~m}, 1 \mathrm{H})$, 2.26-2.18 (m, 1H), 2.12-2.08 (m, 1H), 1.73-1.69 (m, 1H) ppm; ${ }^{13} \mathrm{C}$ NMR $\left(100 \mathrm{MHz}, \mathrm{CDCl}_{3}\right) \delta: 167.2,166.2,159.4$, 
132.3, 104.4, 64.9, 64.8, 63.9, 52.8, 51.6, 36.1, 30.2 ppm; IR (Neat): 2954, 2853, 1778, 1712, 1625, 1436, 1252, 1165 , 1101, 1010, $843 \mathrm{~cm}^{-1}$; HRMS (ESI) $\mathrm{m} / z$ : $[\mathrm{M}+\mathrm{H}]^{+}$calcd. for $\mathrm{C}_{13} \mathrm{H}_{18} \mathrm{NO}_{6}:$ 284.1129; Found: 284.1132.

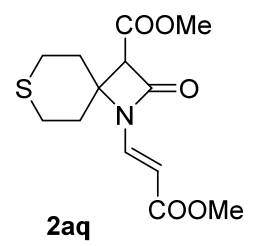

Methyl (E)-1-(3-methoxy-3-oxoprop-1-en-1-yl)-2-oxo-7-thia-1-azaspiro[3.5]nonane-3-carboxylate

(2aq).

According to the general procedure, $\beta$-lactam 2 aq was obtained from tetrahydro- $4 H$-thiopyran-4-one oxime (39.3 mg, $0.3 \mathrm{mmol})$ and methyl propiolate $(76.5 \mathrm{mg}, 0.9 \mathrm{mmol})$ as light yellow oil $(64.6 \mathrm{mg}, 72 \%$ yield; eluent: PE/EtOAc $=6: 1$ to $3: 1) .{ }^{1} \mathrm{H}$ NMR $\left(400 \mathrm{MHz}, \mathrm{CDCl}_{3}\right) \delta: 7.24-7.21(\mathrm{~d}, J=10.2 \mathrm{~Hz}, 1 \mathrm{H}), 5.85-5.81(\mathrm{~d}, J=14.6 \mathrm{~Hz}, 1 \mathrm{H}), 3.78(\mathrm{~s}, 1 \mathrm{H})$, $3.73(\mathrm{~s}, 3 \mathrm{H}), 3.67(\mathrm{~s}, 3 \mathrm{H}), 2.79-2.67(\mathrm{~m}, 2 \mathrm{H}), 2.57-2.53(\mathrm{~m}, 2 \mathrm{H}), 2.47-2.42(\mathrm{~m}, 1 \mathrm{H}), 2.37-2.30(\mathrm{~m}, 1 \mathrm{H}), 2.21-2.14(\mathrm{~m}$, 1H), 2.05-2.00 (m, 1H) ppm; ${ }^{13} \mathrm{C}$ NMR (100 MHz, $\left.\mathrm{CDCl}_{3}\right) \delta: 167.1,166.1,159.4,132.3,104.3,63.7,63.6,52.8,51.6$, 37.6, 31.5, 26.4, 25.8 ppm; IR (Neat): 2951, 2849, 2257, 1774, 1707, 1624, 1434, 1250, 1154, 1026, 957, 913, 844, 727, 647, $585 \mathrm{~cm}^{-1}$; HRMS (ESI) $\mathrm{m} / z$ : $[\mathrm{M}+\mathrm{Na}]^{+}$calcd. for $\mathrm{C}_{13} \mathrm{H}_{17} \mathrm{NNaO}_{5} \mathrm{~S}: 322.0720$; Found: 322.0716

\section{Control experiment using copper acetylide}

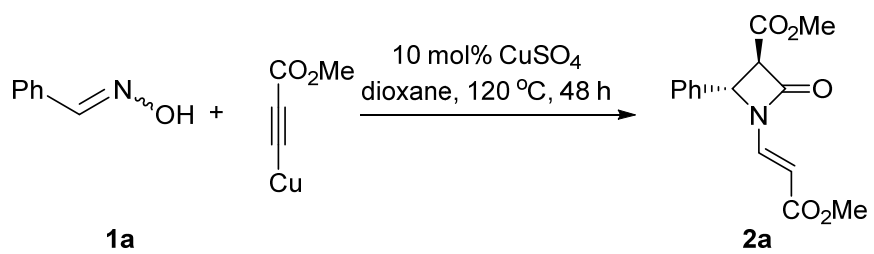

A mixture containing oxime 1a (0.3 mmol, 1.0 equiv), (3-methoxy-3-oxoprop-1-yn-1-yl)copper ${ }^{12}$ (0.9 mmol, 3.0 equiv), anhydrous $\mathrm{CuSO}_{4}\left(0.03 \mathrm{mmol}, 0.1\right.$ equiv) and dioxane $(3 \mathrm{~mL})$ was stirred in a sealed tube at $120^{\circ} \mathrm{C}$ for $48 \mathrm{~h}$ on a heating mantle. After cooling, saturated aqueous $\mathrm{NH}_{4} \mathrm{Cl}$ solution $(5 \mathrm{~mL})$ was added and the resulting mixture was diluted with ethyl acetate $(5 \mathrm{~mL})$. The organic layer was separated and the aqueous layer was extracted with EtOAc $(5$ $\mathrm{mL} \times 3)$. The combined extracts were washed with brine $(10 \mathrm{~mL})$, dried over anhydrous $\mathrm{Na}_{2} \mathrm{SO}_{4}$. After filtration, the filtrate was concentrated under vacuum and the obtained residue was purified by flash column chromatography on silica gel (petroleum ether/EtOAc as eluent, the ratio varies from 10:1 to 4:1) to afford $\beta$-lactam 2a as light yellow oil (37.4 mg, 43\% yield, 91/9 dr).

\section{Gram scale reaction}

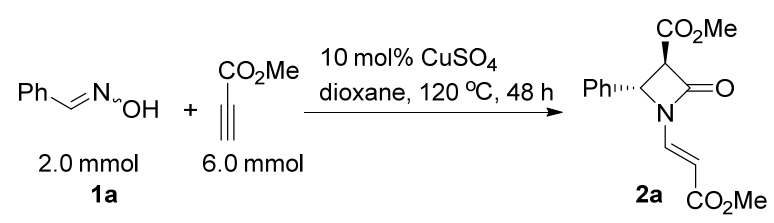

A mixture containing benzaldehyde oxime (242 mg, $2.0 \mathrm{mmol}, 1.0$ equiv), methyl propiolate $(510 \mathrm{mg}, 6.0 \mathrm{mmol}$, 3.0 equiv), anhydrous $\mathrm{CuSO}_{4}\left(0.2 \mathrm{mmol}, 0.1\right.$ equiv) and dioxane $(10 \mathrm{~mL})$ was stirred in a sealed tube at $120{ }^{\circ} \mathrm{C}$ for 48 $\mathrm{h}$ on a heating mantle. After cooling, saturated aqueous $\mathrm{NH}_{4} \mathrm{Cl}$ solution $(10 \mathrm{~mL})$ was added and the resulting mixture was diluted with ethyl acetate $(10 \mathrm{~mL})$. The organic layer was separated and the aqueous layer was extracted with EtOAc $(10 \mathrm{~mL} \times 3)$. The combined extracts were washed with brine $(10 \mathrm{~mL})$, dried over anhydrous $\mathrm{Na}_{2} \mathrm{SO}_{4}$. After filtration, the filtrate was concentrated under vacuum and the obtained residue was purified by flash column 
chromatography on silica gel (petroleum ether/EtOAc as eluent, the ratio varies from 10:1 to 4:1) to afford $\beta$-lactam 2a as light yellow oil (416 mg, 72\% yield, 91/9 dr).

\section{Procedures and data for compounds 3-7}

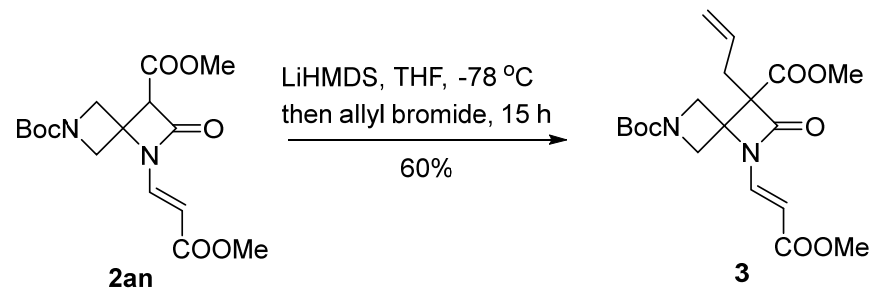

6-(tert-Butyl) 3-methyl (E)-3-allyl-1-(3-methoxy-3-oxoprop-1-en-1-yl)-2-oxo-1,6-diazaspiro[3.3]heptane-3,6dicarboxylate (3). Under argon atmosphere, a solution of LiHMDS ( $0.15 \mathrm{~mL}, 0.15 \mathrm{mmol}, 1.5$ equiv.) in THF was added dropwise to a solution containing 2 an $\left(35.4 \mathrm{mg}, 0.1 \mathrm{mmol}, 1.0\right.$ equiv.) and THF $(3 \mathrm{~mL})$ at $-78{ }^{\circ} \mathrm{C}$, the mixture was kept stirring at $-78{ }^{\circ} \mathrm{C}$ for one hour. Allyl bromide $(0.3 \mathrm{~g}, 0.5 \mathrm{mmol}, 5.0$ equiv. $)$ was then added and the solution was slowly warmed to $20{ }^{\circ} \mathrm{C}$, and was kept at $20{ }^{\circ} \mathrm{C}$ for 15 hours. Then, $10 \% \mathrm{NH}_{4} \mathrm{Cl}$ aqueous solution $(2.0 \mathrm{~mL})$ and $\mathrm{CH}_{2} \mathrm{Cl}_{2}(10.0 \mathrm{~mL})$ were added. The organic phase was separated and washed with water $(3 \times 5 \mathrm{~mL})$, dried over $\mathrm{MgSO}_{4}$. After filtration, the filtrate was concentrated under vacuum. The obtained residue was purified by column chromatography on silica gel to afford 3 as a yellow oil (23.6 mg, 60\% yield; eluent: PE/EtOAc $=6: 1$ to 3:1). ${ }^{1} \mathrm{H}$ NMR $\left(400 \mathrm{MHz}, \mathrm{CDCl}_{3}\right) \delta: 7.61-7.58(\mathrm{~d}, J=14.7 \mathrm{~Hz}, 1 \mathrm{H}), 5.89-5.86(\mathrm{~d}, J=14.7 \mathrm{~Hz}, 1 \mathrm{H}), 5.78-5.68(\mathrm{~m}, 1 \mathrm{H}), 5.28-5.21(\mathrm{~m}$, $2 \mathrm{H}), 4.31-4.22(\mathrm{~m}, 2 \mathrm{H}), 4.15-4.08(\mathrm{~m}, 2 \mathrm{H}), 3.80(\mathrm{~s}, 3 \mathrm{H}), 3.77(\mathrm{~s}, 3 \mathrm{H}), 2.92-2.59(\mathrm{~m}, 2 \mathrm{H}), 1.48(\mathrm{~s}, 9 \mathrm{H}) \mathrm{ppm} ;{ }^{13} \mathrm{C} \mathrm{NMR}$ $\left(100 \mathrm{MHz}, \mathrm{CDCl}_{3}\right) \delta: 167.5,167.0,161.5,156.2,131.6,130.2,120.9,103.0,81.2,68.3,63.0,54.7,53.1,51.8,33.3$, 28.3 ppm; IR (Neat): $\quad 2954,1791,1713,1634,1549,1432,1395,1252,1167,968,850,734,541 \mathrm{~cm}^{-1}$; HRMS (ESI) $m / z:[\mathrm{M}+\mathrm{H}]^{+}$calcd. for $\mathrm{C}_{19} \mathrm{H}_{27} \mathrm{~N}_{2} \mathrm{O}_{7}: 395.1813$; Found: 395.1817 .
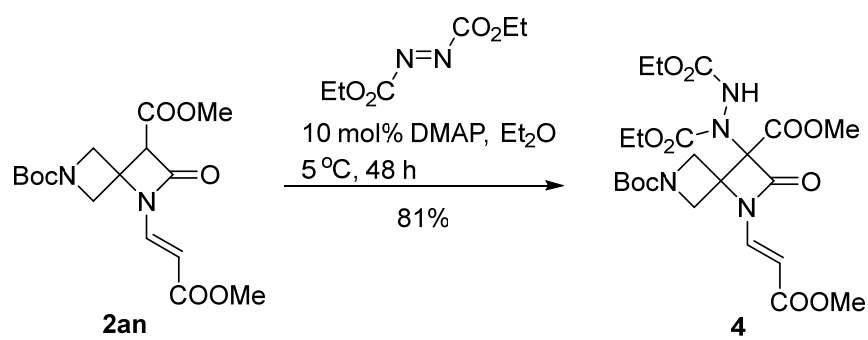

6-(tert-Butyl) 3-methyl (E)-3-(1,2-bis(ethoxycarbonyl)hydrazinyl)-1-(3-methoxy-3-oxoprop-1-en-1-yl)-2-oxo-1,6diazaspiro[3.3] heptane-3,6-dicarboxylate (4). 2 an (35.4 $\mathrm{mg}, 0.1 \mathrm{mmol}, 1.0$ equiv.) was added to a flask containing diethyl (E)-diazene-1,2-dicarboxylate (19.4 mg, $0.11 \mathrm{mmol}, 1.1$ equiv.), DMAP (1.7 mg, $10 \mathrm{~mol} \%)$ and $\mathrm{Et}_{2} \mathrm{O}(1 \mathrm{~mL})$. The reaction mixture was stirred for $48 \mathrm{~h}$ at room temperature. Upon completion, the solvent was removed under vacuum. The obtained residue was purified by flash column chromatography (PE/EtOAc $=4: 1$ to 3:1) to yield 4 as a white solid (42.5 mg, 81\% yield; eluent: PE/EtOAc $=10: 1$ to 4:1). ${ }^{1} \mathrm{H}$ NMR $\left(400 \mathrm{MHz}, \mathrm{CDCl}_{3}\right) \delta: 7.60-7.57(\mathrm{~d}, J=$ $14.7 \mathrm{~Hz}, 1 \mathrm{H}), 7.04-6.99(\mathrm{~m}, 1 \mathrm{H}), 5.99-5.95(\mathrm{~d}, J=14.7 \mathrm{~Hz}, 1 \mathrm{H}), 4.57-4.54(\mathrm{~d}, J=11.4 \mathrm{~Hz}, 1 \mathrm{H}), 4.36-4.15(\mathrm{~m}, 8 \mathrm{H})$, $3.84(\mathrm{~s}, 3 \mathrm{H}), 3.78$ (s, 3H), $1.48(\mathrm{~s}, 9 \mathrm{H}), 1.34-1.25(\mathrm{~m}, 6 \mathrm{H}) \mathrm{ppm} ;{ }^{13} \mathrm{C} \mathrm{NMR}\left(100 \mathrm{MHz}, \mathrm{CDCl}_{3}\right) \delta: 166.3,164.6,156.9$, 156.2, 155.6, 153.8, 131.4, 104.3, 80.6, 80.2, 65.7, 63.4, 62.4, 53.8, 51.7, 28.1, 14.2 ppm; IR (Neat): 2954, 2877, 1791, 
$1765,1723,1694,1634,1549,1450,1432,1395,1251,1168,972,851,743,540 \mathrm{~cm}^{-1}$; HRMS (ESI) $m / z:[\mathrm{M}+\mathrm{H}]^{+}$ calcd. for $\mathrm{C}_{22} \mathrm{H}_{33} \mathrm{~N}_{4} \mathrm{O}_{11}$ : 529.2140; Found: 529.2144.

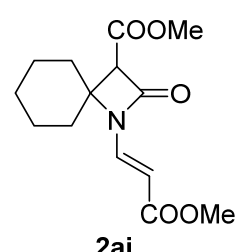

2a

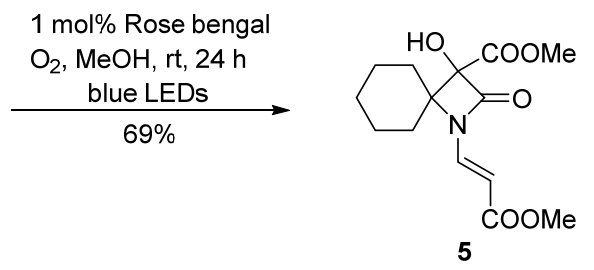

Methyl (E)-3-hydroxy-1-(3-methoxy-3-oxoprop-1-en-1-yl)-2-oxo-1-azaspiro[3.5]nonane-3-carboxylate (5). Under $\mathrm{O}_{2}$ atmosphere, Rose Bengal (1.0 mg, $1 \mathrm{~mol} \%$ ) was added to an oven dried Schlenk tube fitted with a rubber septum containing 2ai (27.9 mg, $0.07 \mathrm{mmol}, 1.0$ equiv.) and $\mathrm{MeOH}(1 \mathrm{~mL})$ at room temperature. The reaction was irradiated by blue LEDs for 24 hours. After completion, the solution was concentrated under vacuum. The obtained residue was purified by flash chromatography to afford 5 as light yellow oil $(20 \mathrm{mg}, 69 \%$ yield, $91 / 9 \mathrm{dr}$; eluent: PE/EtOAc $=2: 1)$. ${ }^{1} \mathrm{H}$ NMR (400 MHz, $\left.\mathrm{CDCl}_{3}\right) \delta$ : 7.30-7.27 (d, $\left.J=14.5 \mathrm{~Hz}, 1 \mathrm{H}\right), 6.01-5.97$ (d, $\left.J=14.5 \mathrm{~Hz}, 1 \mathrm{H}\right), 3.96$ (brs, $\left.1 \mathrm{H}\right), 3.92$ (s, $3 \mathrm{H}), 3.74(\mathrm{~s}, 3 \mathrm{H}), 2.35-2.20(\mathrm{~m}, 1 \mathrm{H}), 2.12-2.08(\mathrm{~m}, 1 \mathrm{H}), 1.89-1.79(\mathrm{~m}, 2 \mathrm{H}), 1.76-1.73(\mathrm{~m}, 2 \mathrm{H}), 1.72-1.66(\mathrm{~m}, 1 \mathrm{H})$, 1.54-1.47 (m, 1H), 1.26-1.06 (m, 2H) ppm; ${ }^{13} \mathrm{C}$ NMR (100 MHz, $\left.\mathrm{CDCl}_{3}\right) \delta: 170.2,167.4,163.2,132.8,105.1,88.6$, 70.6, 53.7, 51.6, 31.2, 30.2, 24.6, 23.6, 23.5 ppm; IR (Neat): 3645, 3036, 2953, 1759, 1732, 1641, 1530, 1458, 1287, 1164, 961, 835, 742, $450 \mathrm{~cm}^{-1}$; HRMS (ESI) $\mathrm{m} / \mathrm{z}$ : $[\mathrm{M}+\mathrm{H}]^{+}$calcd. for $\mathrm{C}_{14} \mathrm{H}_{20} \mathrm{NO}_{6}: 298.1285$; Found: 298.1283 .

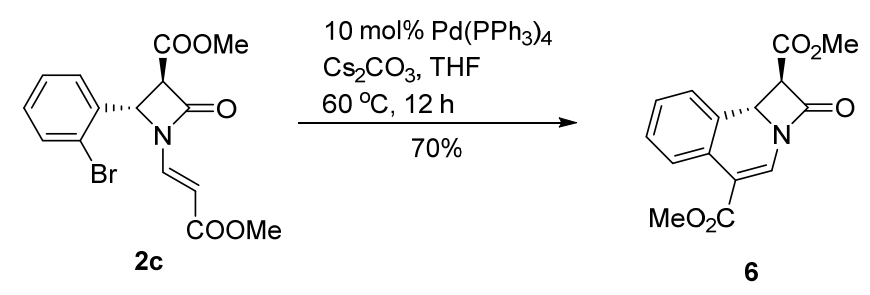

$( \pm) \quad$ Methyl (1R,9bS)-5-((methylperoxy)-12-methyl)-2-oxo-1,9b-dihydro-2H-azeto[2,1-a]isoquinoline-1carboxylate (6). Under nitrogen atmosphere, to an oven dried Schlenk tube fitted with a rubber septum were added $\mathbf{2 c}$ (18.3 mg, 0.05 mmol, 1.0 equiv.) $\mathrm{Pd}\left(\mathrm{PPh}_{3}\right)_{4}$ (11.6 mg, $20 \mathrm{~mol} \%$ ), $\mathrm{Cs}_{2} \mathrm{CO}_{3}(24.4 \mathrm{mg}, 1.5$ equiv.) and anhydrous THF (1 $\mathrm{mL}$ ) at room temperature. The reaction mixture was stirred and heated at $60^{\circ} \mathrm{C}$ for 12 hours. After cooling, the mixture was diluted with saturated aqueous $\mathrm{NH}_{4} \mathrm{Cl}$ solution, and then extracted with ethyl acetate $(10 \mathrm{~mL})$. The organic layer was separated and washed with saturated $\mathrm{NaCl}$ solution, and then dried with anhydrous $\mathrm{Na}_{2} \mathrm{SO}_{4}$. After filtration, the solution was concentrated under vacuum. The obtained residue was purified by flash chromatography to afford $\mathbf{6}$ as a white solid (10 mg, 70\% yield; eluent: PE/EtOAc $=1: 1) .{ }^{1} \mathrm{H}$ NMR (400 MHz, DMSO- $\left.d_{6}\right) \delta: 8.33(\mathrm{~s}, 1 \mathrm{H}), 7.71-7.70(\mathrm{~m}$, $1 \mathrm{H}), 7.49-7.43(\mathrm{~m}, 2 \mathrm{H}), 7.35-7.31(\mathrm{~m}, 1 \mathrm{H}), 5.48-5.46(\mathrm{~d}, J=8.7 \mathrm{~Hz}, 1 \mathrm{H}), 3.88-3.86(\mathrm{~d}, J=8.7 \mathrm{~Hz}, 1 \mathrm{H}), 3.61(\mathrm{~s}, 3 \mathrm{H})$, $3.58(\mathrm{~s}, 3 \mathrm{H}) \mathrm{ppm} ;{ }^{13} \mathrm{C}$ NMR (100 MHz, DMSO- $\left.d_{6}\right) \delta: 180.9,168.7,164.8,157.9,135.7,134.0,131.2,129.1,128.9$, 123.3, 98.9, 57.0, 56.5, 52.7, 51.0 ppm; IR (Neat): 2953, 1767, 1741, 1642, 7536, 1471, 1266, 1160, 943, 805, $552 \mathrm{~cm}^{-}$ ${ }^{1}$; HRMS (ESI) $m / z$ : $[\mathrm{M}+\mathrm{H}]^{+}$calcd. for $\mathrm{C}_{15} \mathrm{H}_{14} \mathrm{NO}_{5}$ : 288.0866; Found: 288.0864. 


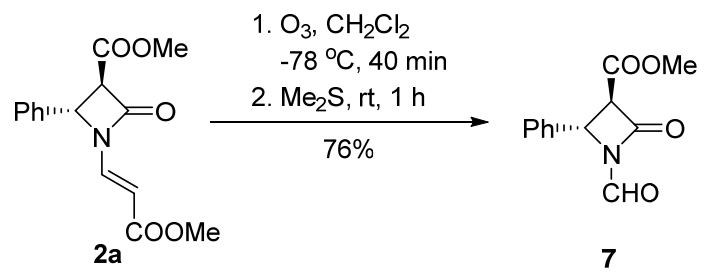

( \pm ) Methyl (3R,4S)-1-formyl-2-oxo-4-phenylazetidine-3-carboxylate (7). At $-78{ }^{\circ} \mathrm{C}$, ozone was bubbled through a solution of $2 \mathrm{a}(1.5 \mathrm{~g}, 5.2 \mathrm{mmol})$ in $\mathrm{CH}_{2} \mathrm{Cl}_{2}(30 \mathrm{~mL})$ for $40 \mathrm{~min}$ until a faint blue color persisted. Then, the ozone was replaced by a stream of dry nitrogen. After treatment with $\mathrm{Me}_{2} \mathrm{~S}(0.2 \mathrm{~mL})$, the solution was allowed to come to room temperature over $1 \mathrm{~h}$. The solution was washed with saturated aqueous $\mathrm{NaCl}(75 \mathrm{~mL})$, dried over $\mathrm{Na}_{2} \mathrm{SO}_{4}$. After filtration, the filtrate was concentrated under vacuum. The residue was rescrystallized with PE to give 7 as a white solid (920 mg, 76\% yield, 96/4 dr). ${ }^{1} \mathrm{H}$ NMR (400 MHz, $\left.\mathrm{CDCl}_{3}\right) \delta: 8.95(\mathrm{~s}, 1 \mathrm{H}), 7.41-7.26(\mathrm{~m}, 5 \mathrm{H}), 5.42-5.41(\mathrm{~d}, J=$ $3.1 \mathrm{~Hz}, 1 \mathrm{H}), 4.11-4.10(\mathrm{~d}, J=3.6 \mathrm{~Hz}, 1 \mathrm{H}), 3.86(\mathrm{~s}, 3 \mathrm{H}) \mathrm{ppm} ;{ }^{13} \mathrm{C} \mathrm{NMR}\left(100 \mathrm{MHz}, \mathrm{CDCl}_{3}\right) \delta: 165.2,160.5,155.9$, 135.1, 129.2, 125.9, 63.0, 56.9, 53.3 ppm; IR (Neat): 2954, 1768, 1724, 1635, 1448, 1259, 1131, $968 \mathrm{~cm}^{-1}$; HRMS (ESI) $m / z:[\mathrm{M}+\mathrm{H}]^{+}$calcd. for $\mathrm{C}_{12} \mathrm{H}_{12} \mathrm{NO}_{4}: 234.0761$; Found: 234.0762 . 


\section{X-Ray Crystallographic Data of Compounds 2g and 2ab}

Compound 2g and 2ab was dissolved in DCM and cold $n$-hexane was added, leaving to slow evaporation overnight to yield colorless needle. A suitable crystal was selected and mounted on a X-ray with Mo radiation ( $\mathrm{a}=10.3244(13)$, 11.4076(15) $\mathrm{c}=16.633(2))$ for cell determination and subsequent data collection at $296 \mathrm{~K}$. Using SHELXL-2014, the structurwas solved with the ShelXT11 13 structure solution program using Intrinsic Phasing and refined with the ShelXL12 $2^{14}$ refinemee package using Least Squares minimisation. The solved structures of $\mathbf{2 g}$ and $\mathbf{2 a b}$ have been deposited in The Cambridge Crystallographic Data Centre (CCDC 1972189 for 2g and CCDC 2036653 for 2ab). The single crystal analysis was carried out using Bruker Smart instrument.
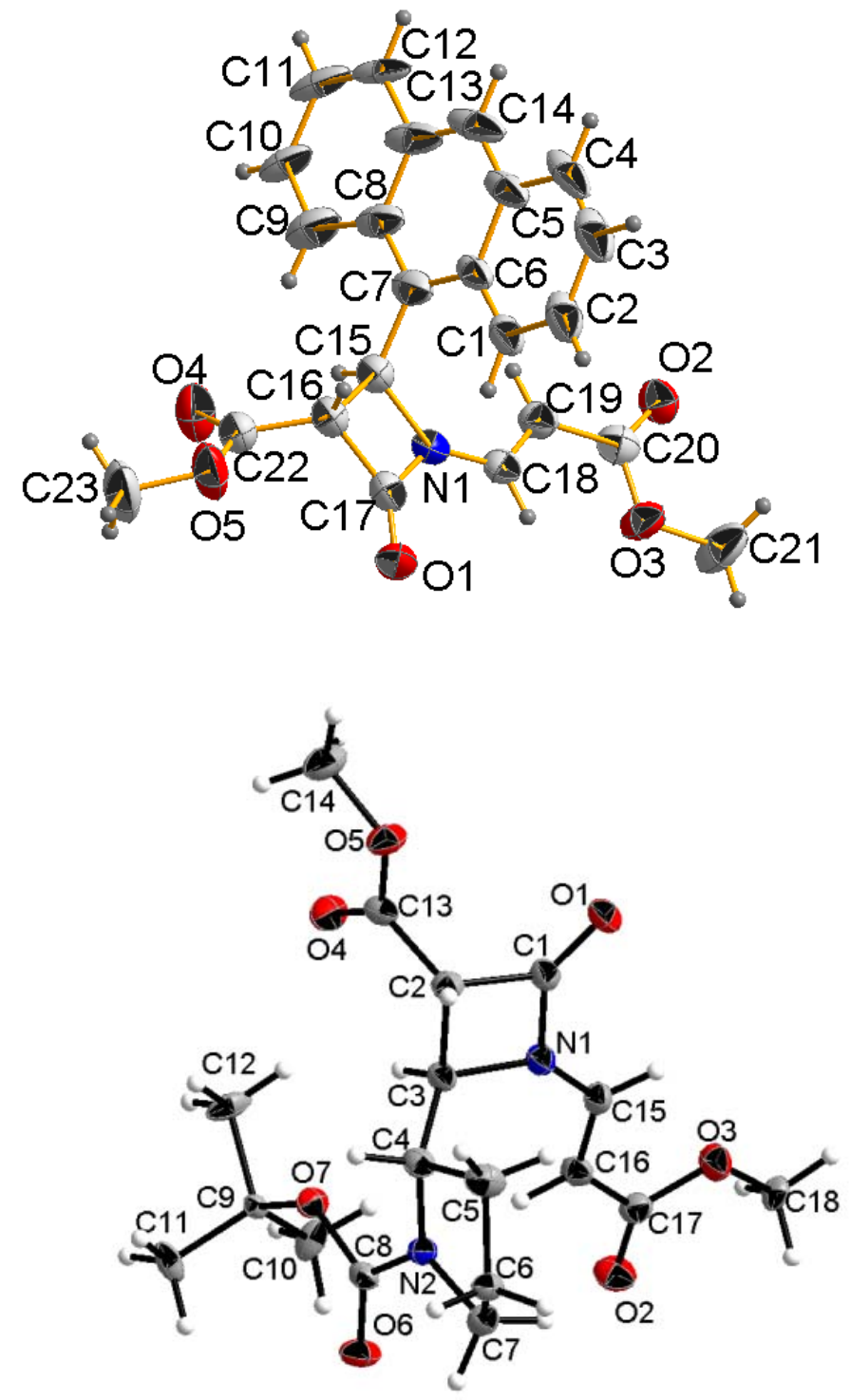

Figure S1. ORTEP diagram of compound $\mathbf{2 g}$ with ellipsoid shown at the 50\% contour percent probability level (top) and ORTEP diagram of compound $\mathbf{2 a b}$ with ellipsoid shown at the 50\% contour percent probability level (bottom). 
Table S1. Crystal Data and Structure Refinement for $\mathbf{2 g}$

\begin{tabular}{|c|c|c|}
\hline Identification code & \multicolumn{2}{|l|}{$2 \mathrm{~g}$} \\
\hline Empirical formula & \multicolumn{2}{|l|}{$\mathrm{C}_{23} \mathrm{H}_{19} \mathrm{NO}_{5}$} \\
\hline Formula weight & \multicolumn{2}{|l|}{389.39} \\
\hline Temperature & \multicolumn{2}{|l|}{$296(2) \mathrm{K}$} \\
\hline Wavelength & \multicolumn{2}{|l|}{$0.71073 \AA$} \\
\hline \multirow[t]{2}{*}{ Crystal system, space group } & \multicolumn{2}{|l|}{ Monoclinic, P2(1)/c } \\
\hline & $\mathrm{a}=14.8214(9) \AA$ & $\alpha=90^{\circ}$ \\
\hline \multirow[t]{2}{*}{ Unit cell dimensions } & $\mathrm{b}=13.5389(10) \AA$ & $\beta=93.354(2)^{\mathrm{o}}$ \\
\hline & $\mathrm{c}=9.5926(5) \AA$ & $\gamma=90^{\circ}$ \\
\hline Volume & \multicolumn{2}{|l|}{$1921.6(2) \AA^{3}$} \\
\hline Z, Calculated density & \multicolumn{2}{|l|}{$4,1.346 \mathrm{Mg} / \mathrm{m}^{3}$} \\
\hline Absorption coefficient & \multicolumn{2}{|l|}{$0.095 \mathrm{~mm}^{-1}$} \\
\hline $\mathrm{F}(000)$ & \multicolumn{2}{|l|}{816} \\
\hline Crystal size & \multicolumn{2}{|l|}{$0.35 \times 0.33 \times 0.3 \mathrm{~mm}$} \\
\hline Theta range for data collection & \multicolumn{2}{|l|}{2.04 to $27.52 \mathrm{deg}$. } \\
\hline Limiting indices & \multicolumn{2}{|c|}{$-19<=\mathrm{h}<=19,-17<=\mathrm{k}<=12,-12<=\mathrm{l}<=12$} \\
\hline Reflections collected / unique & \multicolumn{2}{|c|}{$17721 / 4436[\mathrm{R}(\mathrm{int})=0.0505]$} \\
\hline Completeness to theta $=27.52$ & \multicolumn{2}{|l|}{$99.3 \%$} \\
\hline Absorption correction & \multicolumn{2}{|l|}{ None } \\
\hline Refinement method & \multicolumn{2}{|c|}{ Full-matrix least-squares on $\mathrm{F}^{\wedge} 2$} \\
\hline Data / restraints / parameters & \multicolumn{2}{|l|}{$4406 / 1 / 264$} \\
\hline Goodness-of-fit on $\mathrm{F}^{\wedge} 2$ & \multicolumn{2}{|l|}{1.021} \\
\hline Final $R$ indices $[I>2 \operatorname{sigma}(I)]$ & \multicolumn{2}{|c|}{$\mathrm{R} 1=0.0538, \mathrm{wR} 2=0.1325$} \\
\hline $\mathrm{R}$ indices (all data) & \multicolumn{2}{|c|}{$\mathrm{R} 1=0.0745, \mathrm{wR} 2=0.1477$} \\
\hline Largest diff. peak and hole & \multicolumn{2}{|c|}{0.320 and -0.269 e. $\AA^{-3}$} \\
\hline
\end{tabular}


Table S2. Crystal Data and Structure Refinement for 2 ab.

\begin{tabular}{|c|c|c|}
\hline Identification code & \multicolumn{2}{|l|}{$2 \mathbf{a b}$} \\
\hline Empirical formula & \multicolumn{2}{|l|}{$\mathrm{C}_{18} \mathrm{H}_{26} \mathrm{~N}_{2} \mathrm{O}_{7}$} \\
\hline $\begin{array}{l}\text { Formula weight } \\
\text { Temperature }\end{array}$ & \multicolumn{2}{|l|}{382.41} \\
\hline Wavelength & \multicolumn{2}{|l|}{$0.71073 \AA$} \\
\hline Crystal system & \multicolumn{2}{|l|}{ Orthorhombic } \\
\hline Space group & \multicolumn{2}{|l|}{$\begin{array}{l}\mathrm{P} 22_{12} 2 \\
\mathrm{a}=10.3244(13) \AA\end{array}$} \\
\hline \multirow[t]{2}{*}{ Unit cell dimensions } & $\mathrm{b}=11.4076(15) \AA$ & $\beta=90^{\circ}$ \\
\hline & $c=16.633(2) \AA$ & $\gamma=90^{\circ}$ \\
\hline Volume & \multicolumn{2}{|l|}{$1959.0(4) \AA^{3}$} \\
\hline Z & \multicolumn{2}{|l|}{4} \\
\hline Density (calculated) & \multicolumn{2}{|l|}{$1.297 \mathrm{Mg} / \mathrm{m}^{3}$} \\
\hline Absorption coefficient & \multicolumn{2}{|l|}{$0.100 \mathrm{~mm}^{-1}$} \\
\hline $\mathrm{F}(000)$ & \multicolumn{2}{|l|}{816} \\
\hline Crystal size & \multicolumn{2}{|c|}{$0.3 \times 0.25 \times 0.25 \mathrm{~mm}^{3}$} \\
\hline Theta range for data collection & \multicolumn{2}{|l|}{2.165 to $27.504^{\circ}$. } \\
\hline Index ranges & \multicolumn{2}{|c|}{$-13<=\mathrm{h}<=13,-14<=\mathrm{k}<=14,-21<=\mathrm{l}<=20$} \\
\hline $\begin{array}{l}\text { Reflections collected } \\
\text { Independent reflections }\end{array}$ & \multicolumn{2}{|c|}{$\begin{array}{l}16737 \\
4507[\mathrm{R}(\mathrm{int})=0.0630]\end{array}$} \\
\hline Completeness to theta $=25.242^{\circ}$ & \multicolumn{2}{|l|}{$100.0 \%$} \\
\hline $\begin{array}{l}\text { Absorption correction } \\
\text { Max. and min. transmission }\end{array}$ & \multicolumn{2}{|l|}{$\begin{array}{l}\text { None } \\
0.975 \text { and } 0.970\end{array}$} \\
\hline Refinement method & \multicolumn{2}{|c|}{ Full-matrix least-squares on $\mathrm{F}^{2}$} \\
\hline Data / restraints / parameters & \multicolumn{2}{|l|}{4499 / 0 / 249} \\
\hline $\begin{array}{l}\text { Goodness-of-fit on } \mathrm{F}^{2} \\
\text { Final R indices [I>2sigma(I)] }\end{array}$ & \multicolumn{2}{|c|}{$\begin{array}{l}1.040 \\
\mathrm{R} 1=0.0525, \mathrm{wR} 2=0.1189\end{array}$} \\
\hline $\mathrm{R}$ indices (all data) & \multicolumn{2}{|c|}{$\mathrm{R} 1=0.0864, \mathrm{wR} 2=0.1369$} \\
\hline Absolute structure parameter & \multicolumn{2}{|l|}{$0.2(7)$} \\
\hline Extinction coefficient & \multicolumn{2}{|l|}{$\mathrm{n} / \mathrm{a}$} \\
\hline Largest diff. peak and hole & \multicolumn{2}{|c|}{0.401 and -0.264 e. $\AA^{-3}$} \\
\hline
\end{tabular}

\section{References}

1. Gao, S.; Gao, L.; Meng, H.; Luo, M.; Zeng, X. Iron-catalyzed synthesis of benzoxazoles by oxidative coupling/cyclization of phenolderivatives with benzoyl aldehyde oximes. Chem. Commun. 2017, 53, 9886-9889.

2. Zhang, G.; Wen, X.; Wang, Y.; Han, X.; Luan, Y.; Zheng, L.; Ding, C.; Cao, X. In situ generation of active species "NO" for the aerobic oxidative deprotection of aldoximes catalyzed by $\mathrm{FeCl}_{3} / \mathrm{TEMPO}$. RSC Adv. 2013, 3, 22918-22921.

3. Goyard, D.; Konya, B.; Chajistamatiou, A. S.; Chrysina, E. D.; Leroy, J.; Balzarin, S.; Tournier, M.; Tousch, D.; Petit, P.; Duret, C.; Maurel, P.; Somsak, L.; Docsa, T.; Gergely, P.; Praly, J.-P.; Azay-Milhau, J.; Vidal, S. 
Glucose-derived spiro-isoxazolines are anti-hyperglycemic agentsagainst type 2 diabetes through glycogen phosphorylase inhibition. Eur. J. Med. Chem. 2016, 108, 444-454.

4. Bi, F.; Song, D.; Zhang, N.; Liu, Z.; Gu, X.; Hu, C.; Cai, X.; Venter, H.; Ma, S. Design, synthesis and structurebased optimization of novel isoxazole-containing benzamide derivatives as FtsZ modulators. Eur. J. Med. Chem. 2018, 159, 90-103.

5. Kalisiak, J.; Ralph, E. C.; Zhang, J.; Cashman, J. R. Amidine-Oximes: Reactivators for Organophosphate Exposure. J. Med. Chem. 2011, 54, 3319-3330.

6. Rizk, T.; E. Bilodeau, J.-F.; Beauchemin, A. M. Synthesis of Pyridines and Pyrazines Using an Intramolecular Hydroamination-Based Reaction Sequence. Angew. Chem. Int. Ed. 2009, 48, 8325-8327.

7. Furnival, R. C.; Saruengkhanphasit, R.; Holberry, H. E.; Shewring, J. R.; Guerrand, H. D. S.; Adams, H.; Coldham, I. Cascade oxime formation, cyclization to a nitrone, and intermolecular dipolar cycloaddition. Org. Biomol. Chem. 2016, 14, 10953-10962.

8. Tran, N. C.; Dhondt, H.; Flipo, M.; Deprez, B.; Willand, N. Synthesis of functionalized 2-isoxazolines as threedimensional fragments for fragment-based drug discovery. Tetrahedron Lett. 2015, 56, 4119-4123.

9. Mo, X.; Morgan, D. R.; Ang, H. T.; Hall, D. G. Scope and Mechanism of a True Organocatalytic Beckmann Rearrangement with a Boronic Acid/Perfluoropinacol System under Ambient Conditions. J. Am. Chem. Soc. 2018, $140,5264-5271$.

10. Ai, W.; Liu, Y.; Wang, Q.; Lu, Z.; Liu, Q. Cu-Catalyzed Redox-Neutral Ring Cleavage of Cycloketone O-Acyl Oximes: Chemodivergent Access to Distal Oxygenated Nitriles. Org. Lett. 2018, 20, 409-412.

11. a) Siitonen, J. H.; Kattamuri, P. V.; Yousufuddin, M.; Kürti, L. Arylboronic Acid-Catalyzed C-Allylation of Unprotected Oximes: Total Synthesis of $\mathrm{N}$-Me-Euphococcine. Org. Lett. 2020, 22, 2486-2489. b) Martyloga, O. V.; Myronenko, A.; Tkachenko, A. M.; Matvienko, V. O.; Kuchkovska, Y. O.; Grygorenko, O. O. Multigram Synthesis of Functionalized Spirocyclic Diazirines. Eur. J. Org. Chem. 2019, 3744-3750. c) Mullen, P.; Miel, H.; McKervey, M. A. N-Boc 4-nitropiperidine: preparation and conversion into a spiropiperidine analogue of the eastern part of maraviroc. Tetrahedron Lett. 2010, 51, 3216-3217. d) Gellibert, F.; Gouville, A.-C.; Woolven, J.; Mathews, N.; Nguyen, X. V.-L.; Bertho-Ruault, C.; Patikis, A.; Grygielko, E. T.; Laping, N. J.; Huet, S. Discovery of 4-\{4-[3-(Pyridin-2-yl)-1H-pyrazol-4-yl]pyridin-2-yl $\} \quad \mathrm{N}$-(tetrahydro-2Hpyran-4-yl)benzamide (GW788388): A Potent, Selective, and Orally Active Transforming Growth Factor-â Type I Receptor Inhibitor. $J$. Med. Chem. 2006, 49, 2210-2221. e) Subramanian, P. K.; Ramalingam, K. Synthesis and Conformational Analysis of Substituted 4-Aminothianes. J. Org. Chem. 1981, 46, 4376-4383.

12. Chen, W.; Wang, B.; Liu, N.; Huang, D.; Wang, X.; Hu, Y. Tandem Synthesis of 3-Halo-5-Substituted Isoxazoles from 1-Copper(I) Alkynes and Dihaloformaldoximes. Org. Lett. 2014, 16, 6140-6143.

13. Sheldrick, G. M. Crystal Structure Refinement with SHELXL. Acta Cryst. Acta Cryst. 2015, A71, 3-8

14. Sheldrick, G. M. Crystal Structure Refinement with SHELXL. Acta Cryst. 2015, C71, 3-8. 


\section{Copies of NMR Spectra}

${ }^{1} \mathrm{H}$ NMR of $2 \mathbf{a}\left(400 \mathrm{MHz}, \mathrm{CDCl}_{3}\right)$
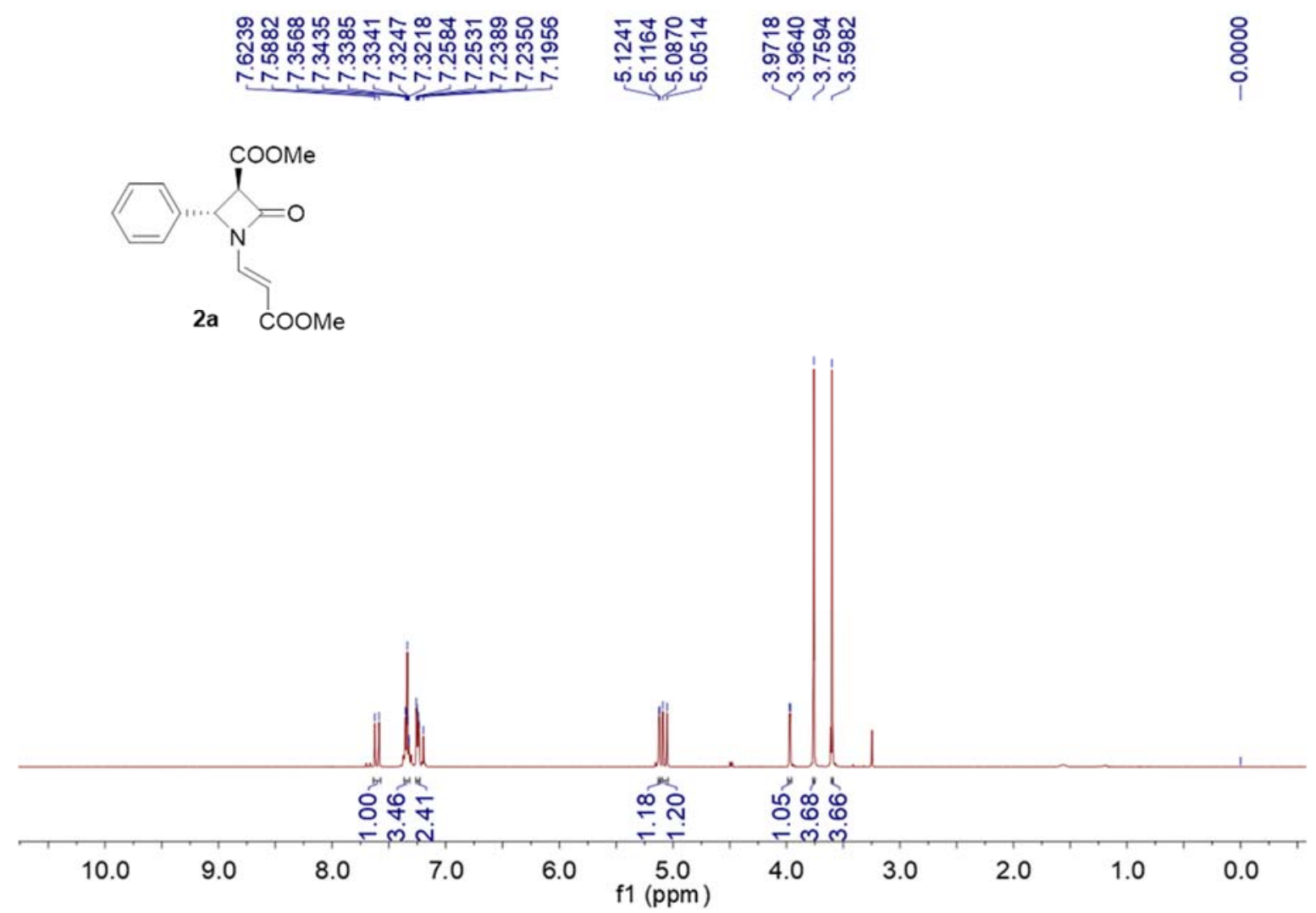

${ }^{13} \mathrm{C}$ NMR of $2 \mathbf{a}\left(100 \mathrm{MHz}, \mathrm{CDCl}_{3}\right)$

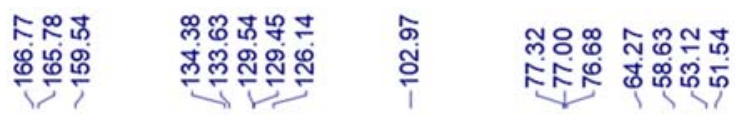
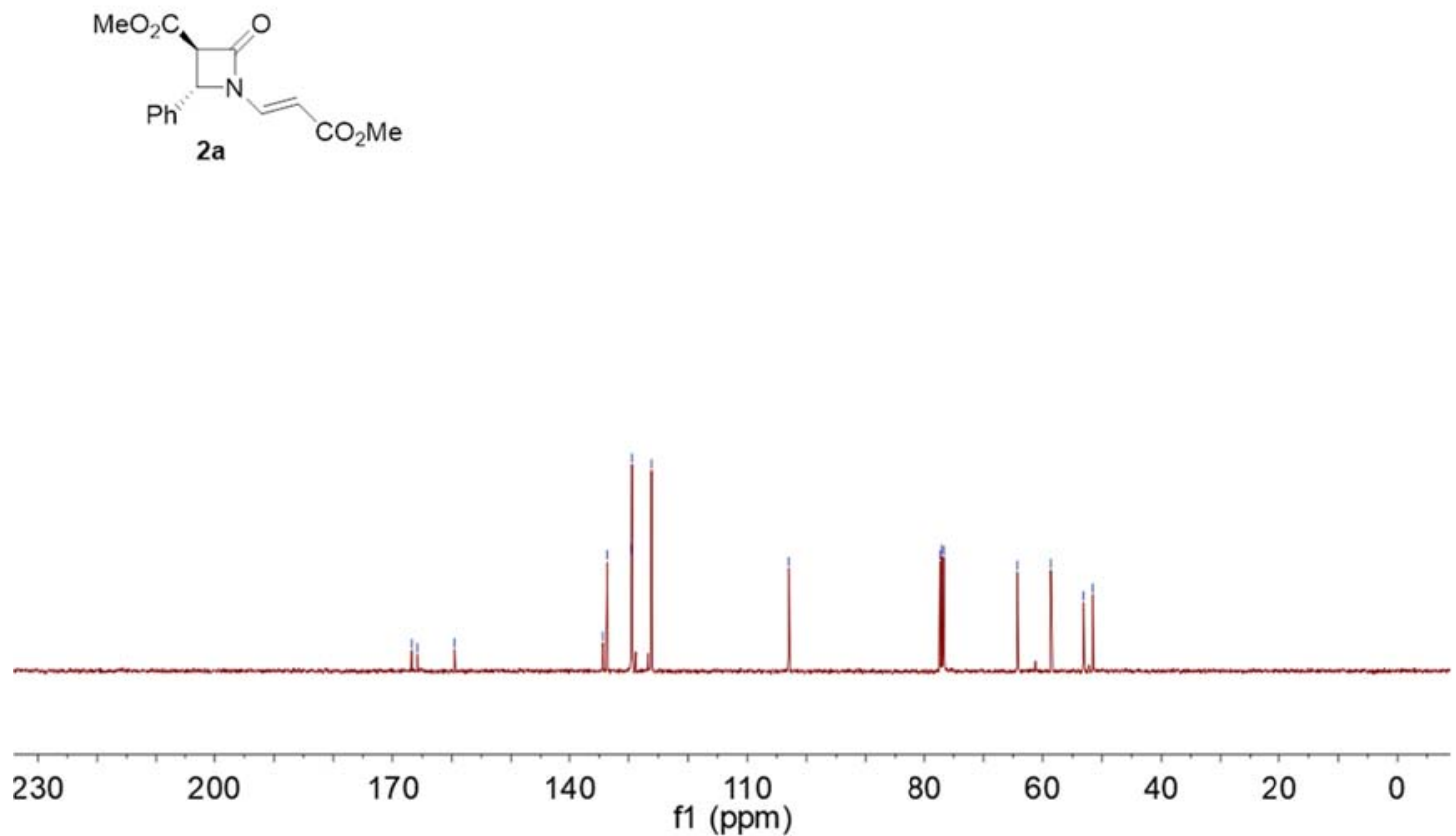
${ }^{1} \mathrm{H}$ NMR of $\mathbf{2 b}\left(400 \mathrm{MHz}, \mathrm{CDCl}_{3}\right)$

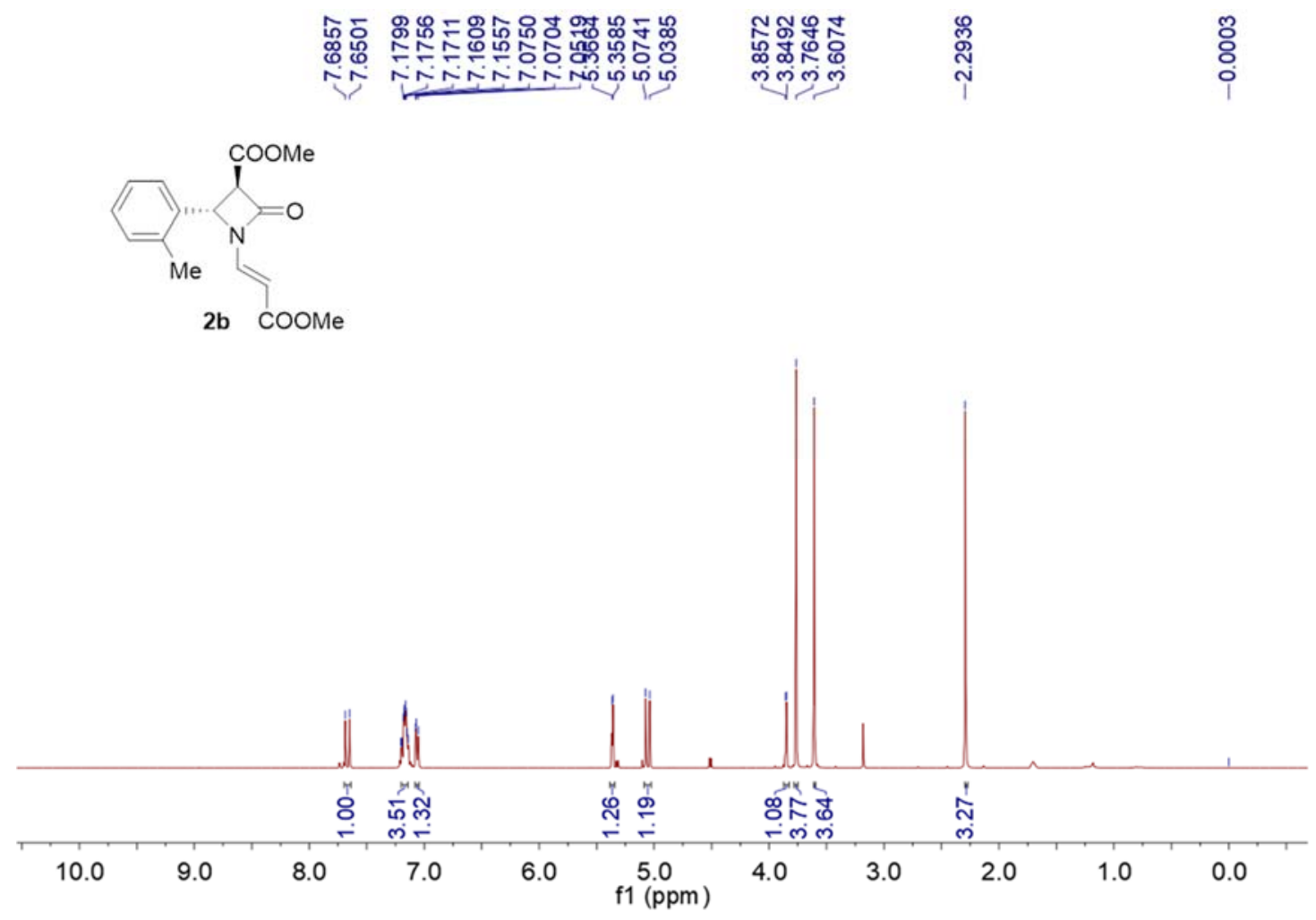

${ }^{13} \mathrm{C}$ NMR of $\mathbf{2 b}\left(100 \mathrm{MHz}, \mathrm{CDCl}_{3}\right)$

\begin{tabular}{|c|c|c|c|c|}
\hline 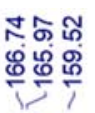 & 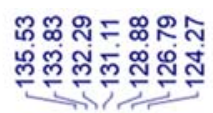 & ֻัญ & 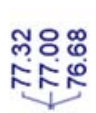 & 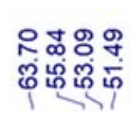 \\
\hline
\end{tabular}
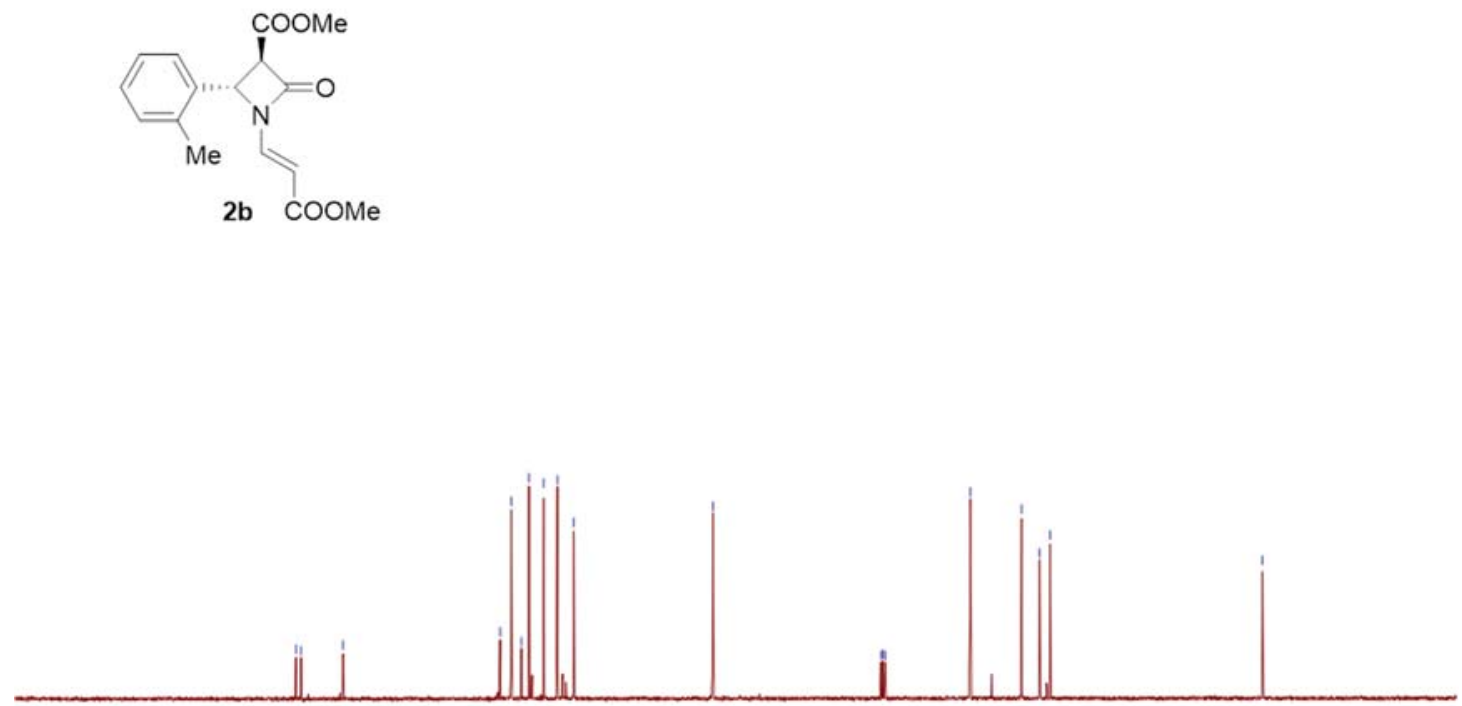

190

170

150

130

${ }_{\mathrm{f} 1(\mathrm{ppm})} 90$

$80 \quad 70 \quad 60$ 
${ }^{1} \mathrm{H}$ NMR of $2 \mathbf{c}\left(400 \mathrm{MHz}, \mathrm{CDCl}_{3}\right)$

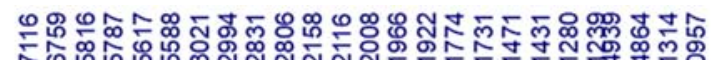

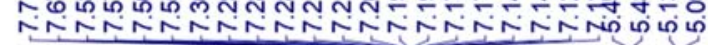

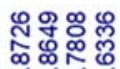

लिंक्ल

:
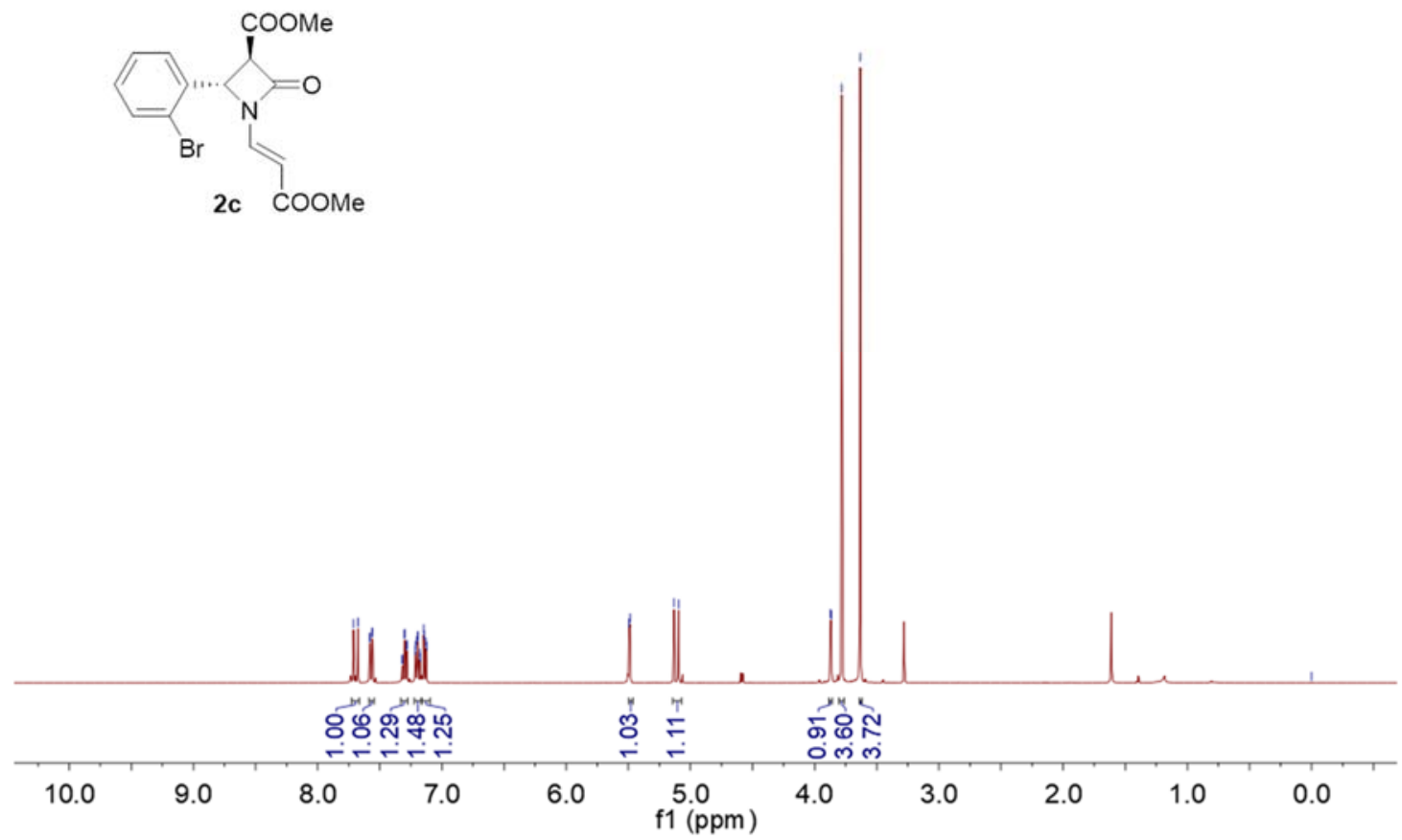

${ }^{13} \mathrm{C}$ NMR of $2 \mathbf{c}\left(100 \mathrm{MHz}, \mathrm{CDCl}_{3}\right)$

\begin{tabular}{|c|c|c|}
\hline ஜ유 & 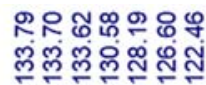 & 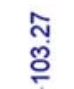 \\
\hline
\end{tabular}
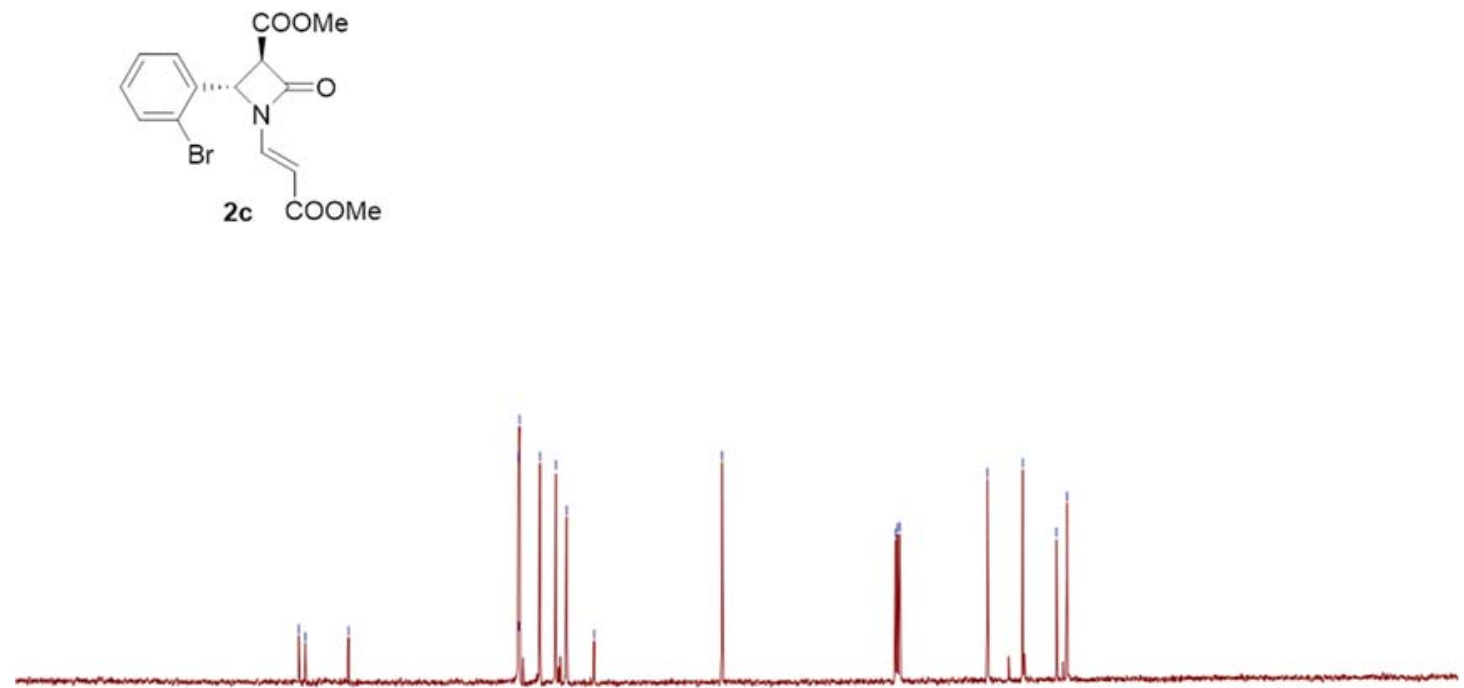

190

170

150

130

110 f1 (ppm)

$\begin{array}{lllllllll}80 & 70 & 60 & 50 & 40 & 30 & 20 & 10 & 0\end{array}$ 
${ }^{1} \mathrm{H}$ NMR of $2 \mathbf{d}\left(400 \mathrm{MHz}, \mathrm{CDCl}_{3}\right)$

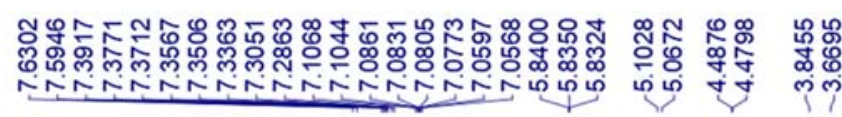

8
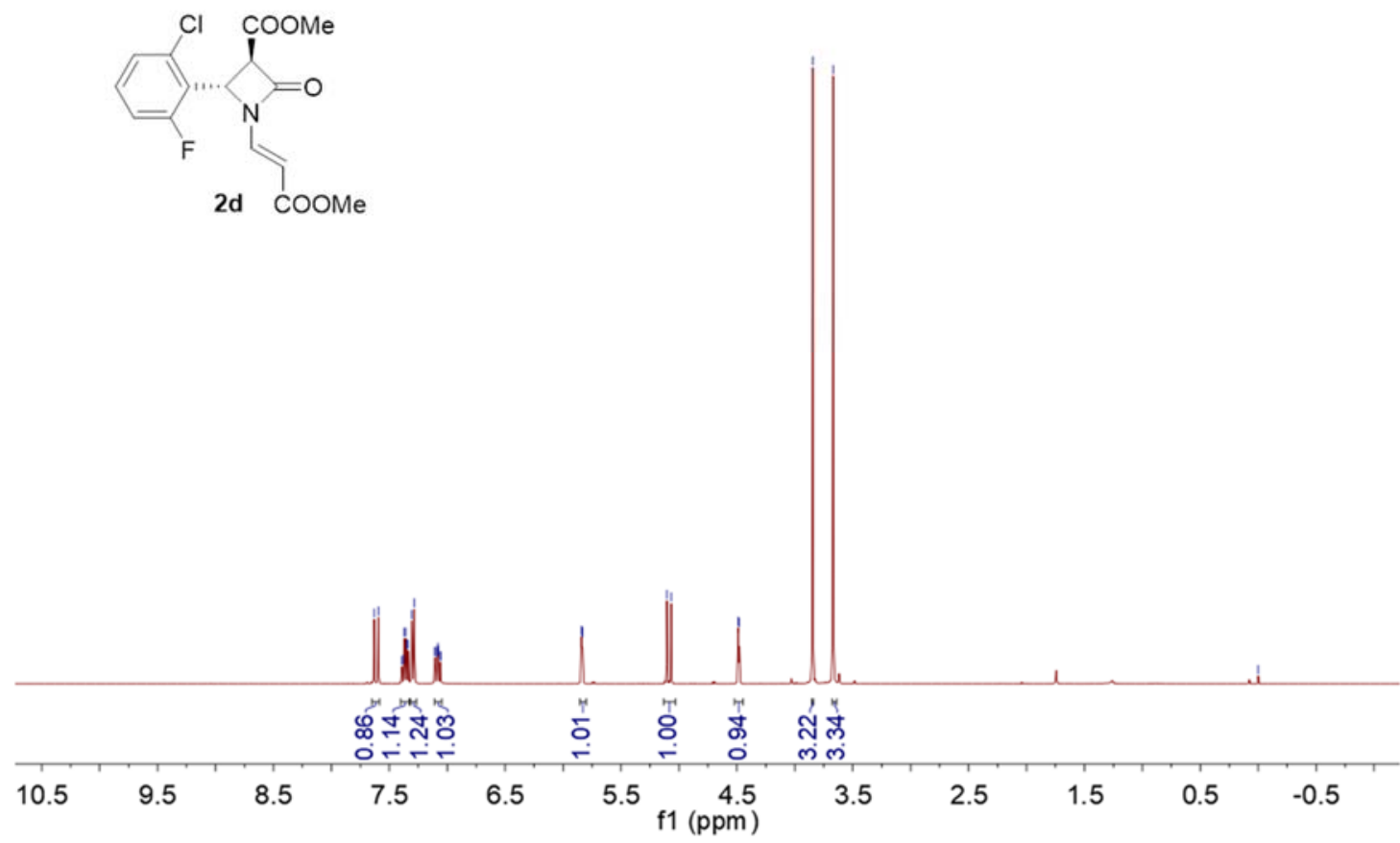

${ }^{13} \mathrm{C}$ NMR of $2 \mathbf{d}\left(100 \mathrm{MHz}, \mathrm{CDCl}_{3}\right)$

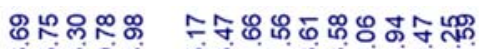

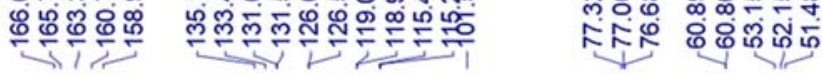

్ㅣㅇㅛ
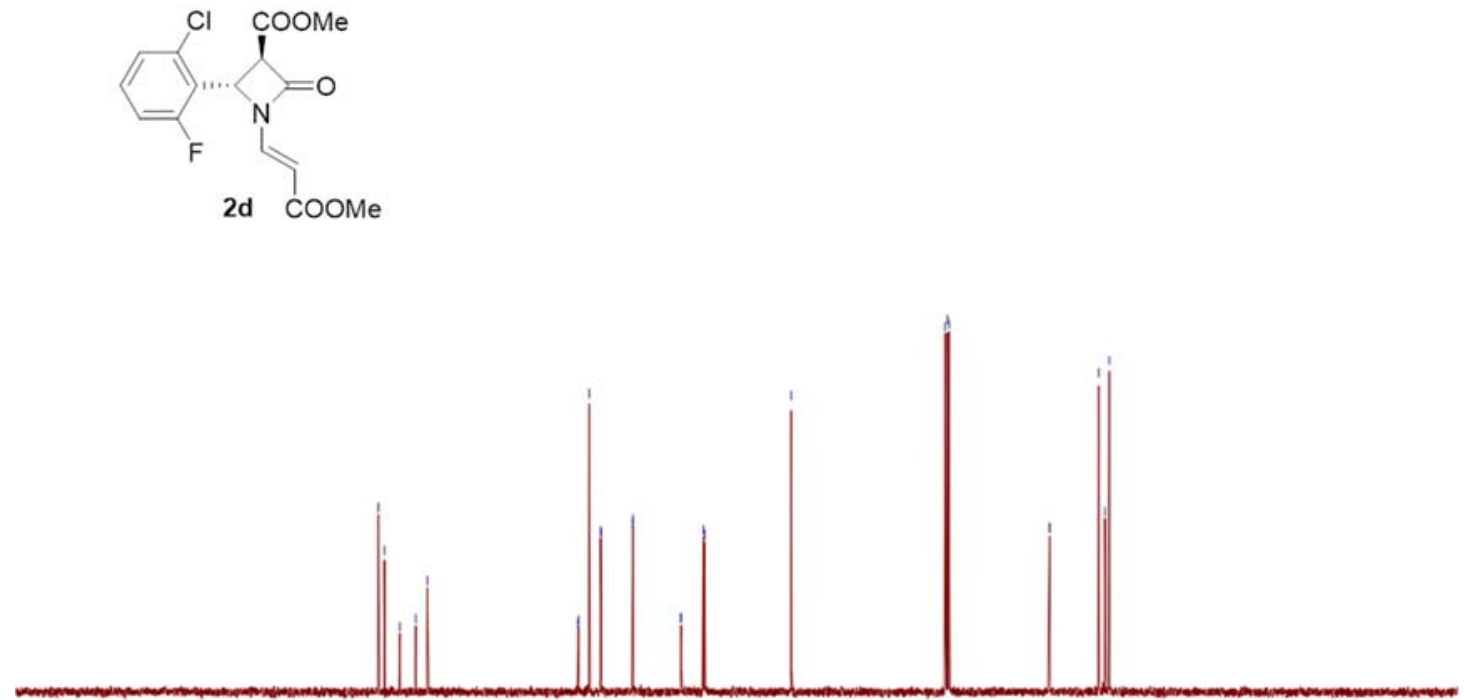

\begin{tabular}{|c|c|c|c|c|c|c|c|c|c|c|c|c|c|c|}
\hline 210 & 190 & 170 & 150 & 130 & $\begin{array}{l}110 \\
\mathrm{f} 1(\mathrm{ppm})\end{array}$ & 90 & 80 & 70 & 60 & 50 & 40 & 3 & & $20 \quad 10 \quad 0$ \\
\hline
\end{tabular}


${ }^{1} \mathrm{H}$ NMR of $2 \mathbf{e}\left(400 \mathrm{MHz}, \mathrm{CDCl}_{3}\right)$
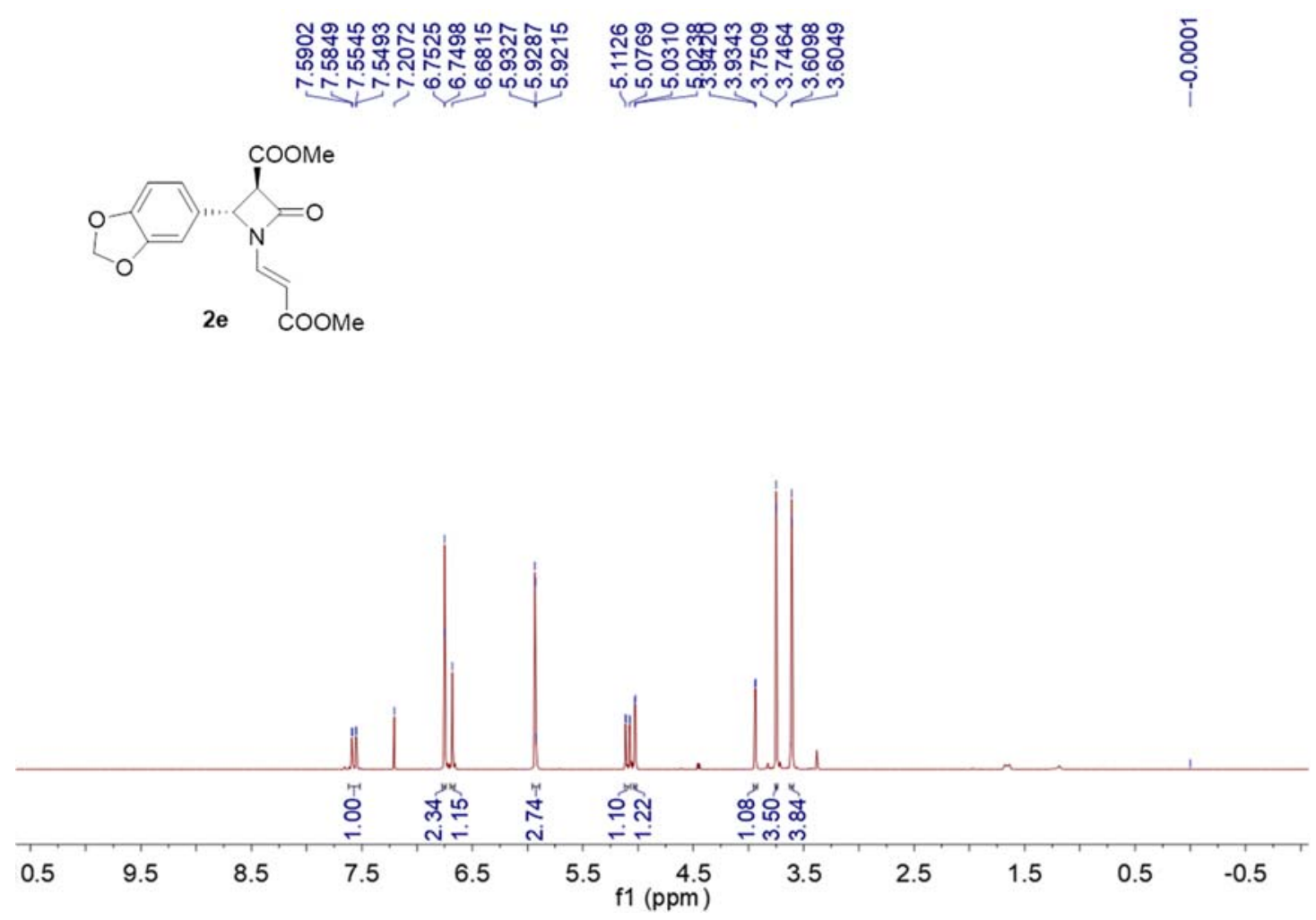

${ }^{13} \mathrm{C}$ NMR of $2 \mathbf{e}\left(100 \mathrm{MHz}, \mathrm{CDCl}_{3}\right)$

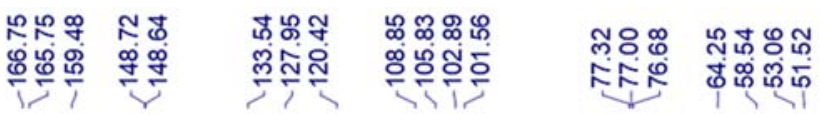
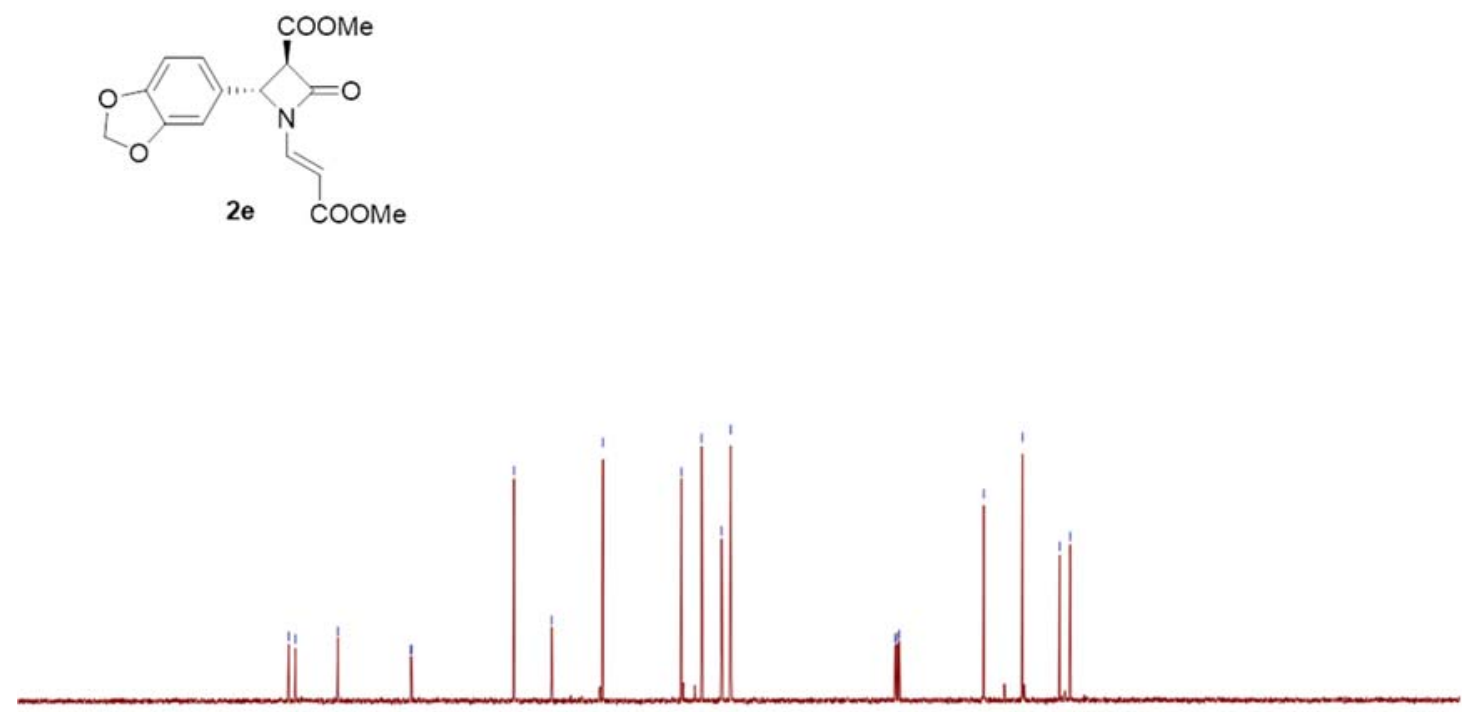

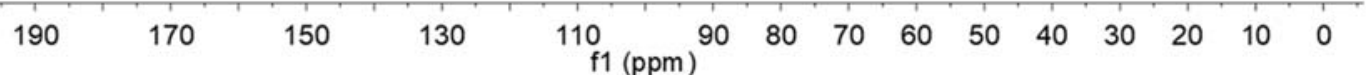


${ }^{1} \mathrm{H}$ NMR of $\mathbf{2 f}\left(400 \mathrm{MHz}, \mathrm{CDCl}_{3}\right)$

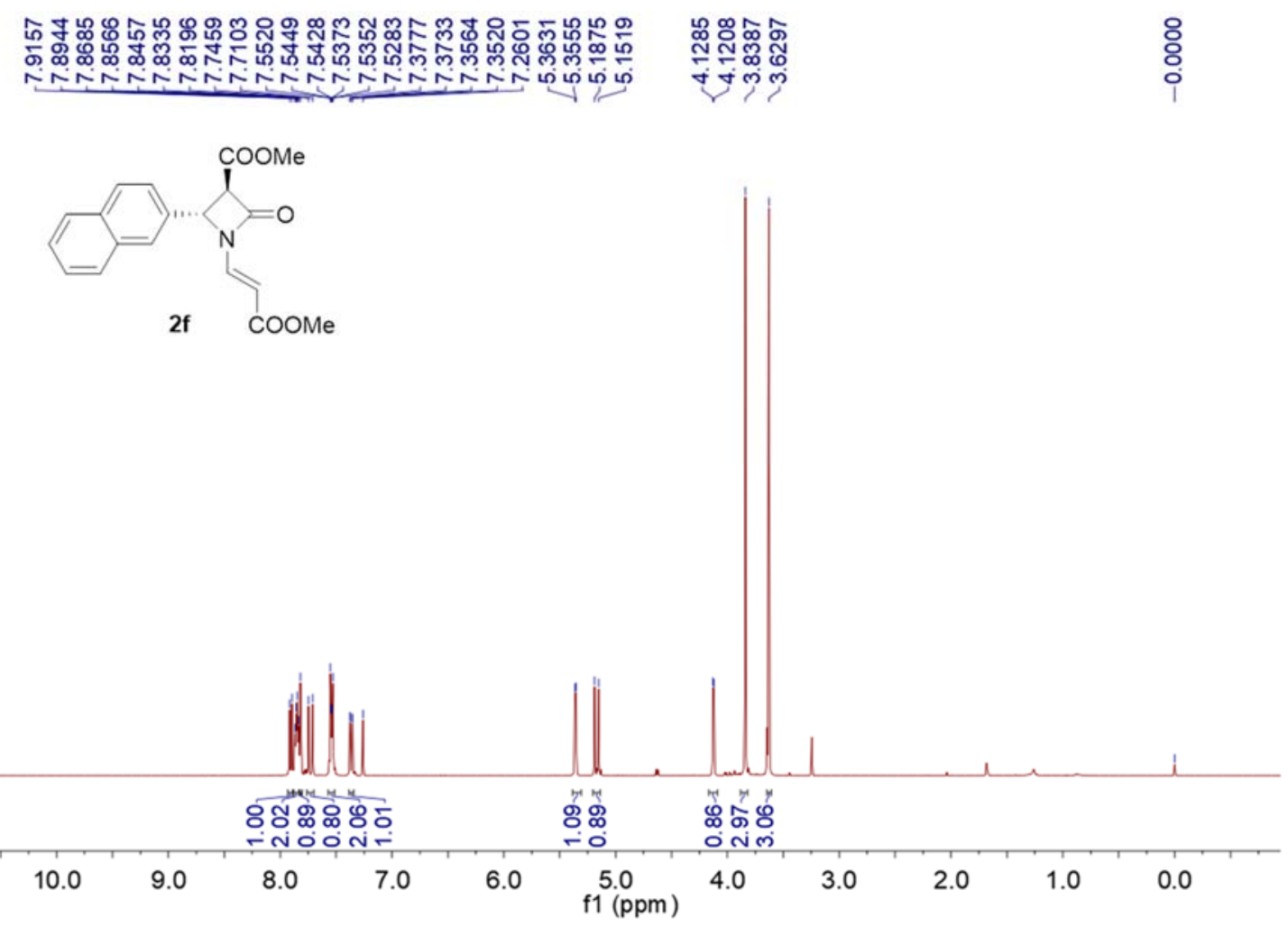

${ }^{13} \mathrm{C}$ NMR of $2 \mathbf{f}\left(100 \mathrm{MHz}, \mathrm{CDCl}_{3}\right)$

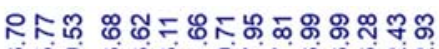

ถำำ

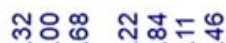

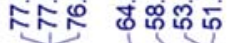
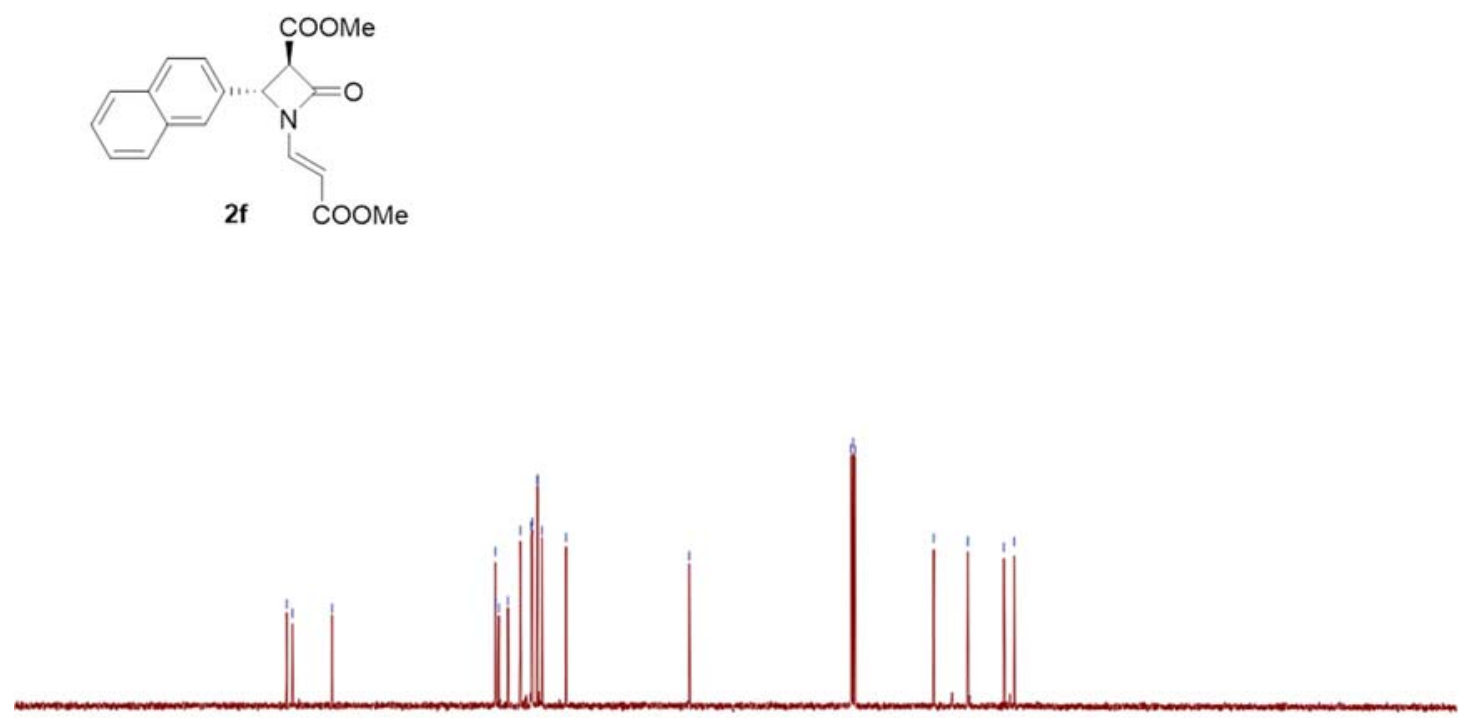

190

170

150

130

110

f1 (ppm)

$\begin{array}{lllllllll}80 & 70 & 60 & 50 & 40 & 30 & 20 & 10 & 0\end{array}$ 
${ }^{1} \mathrm{H}$ NMR of $2 \mathbf{g}\left(400 \mathrm{MHz}, \mathrm{CDCl}_{3}\right)$

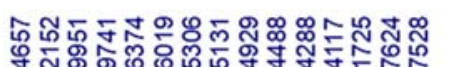

๙ तั

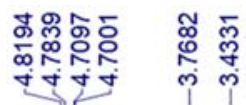

:

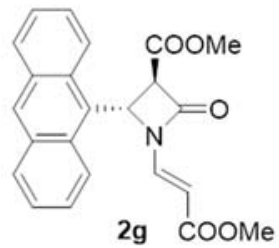

2g coOMe

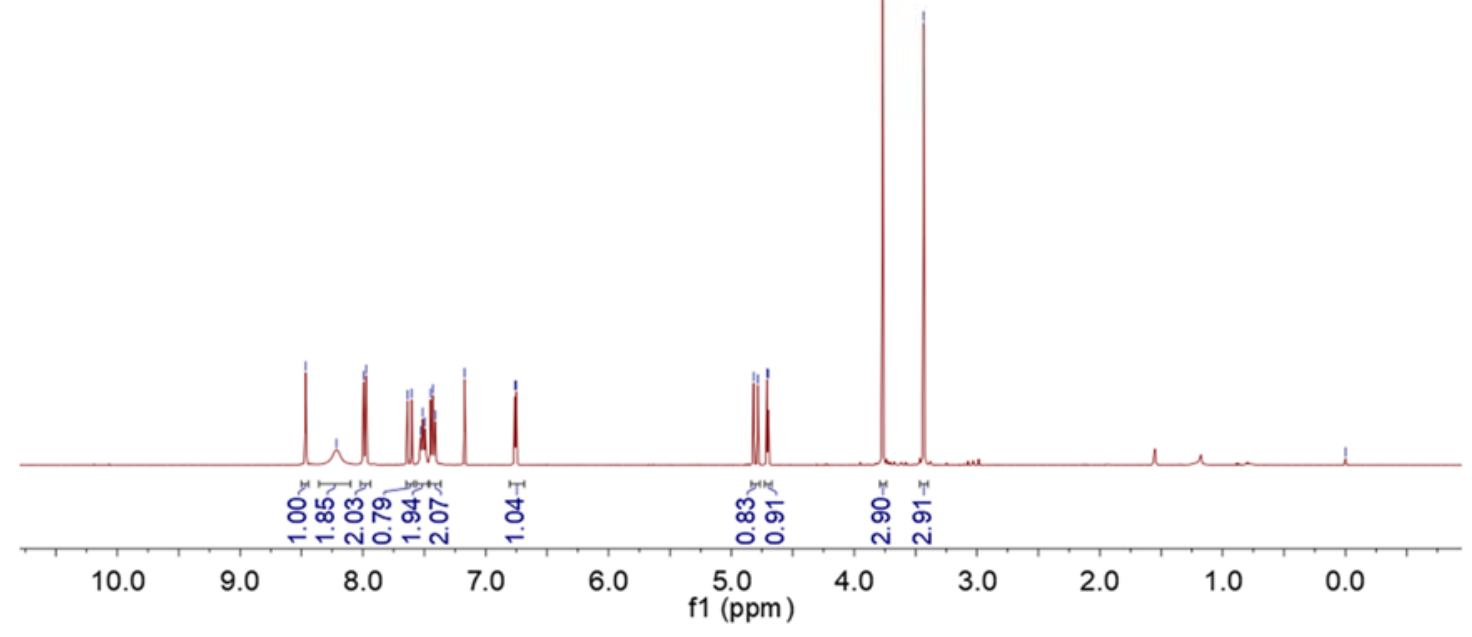

${ }^{13} \mathrm{C}$ NMR of $2 \mathrm{~g}\left(100 \mathrm{MHz}, \mathrm{CDCl}_{3}\right)$

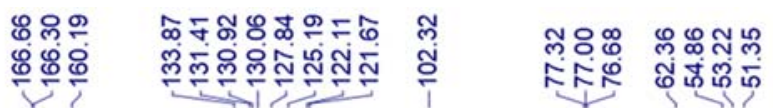

$\sum_{2 \mathrm{~g}}^{\text {coome }}$

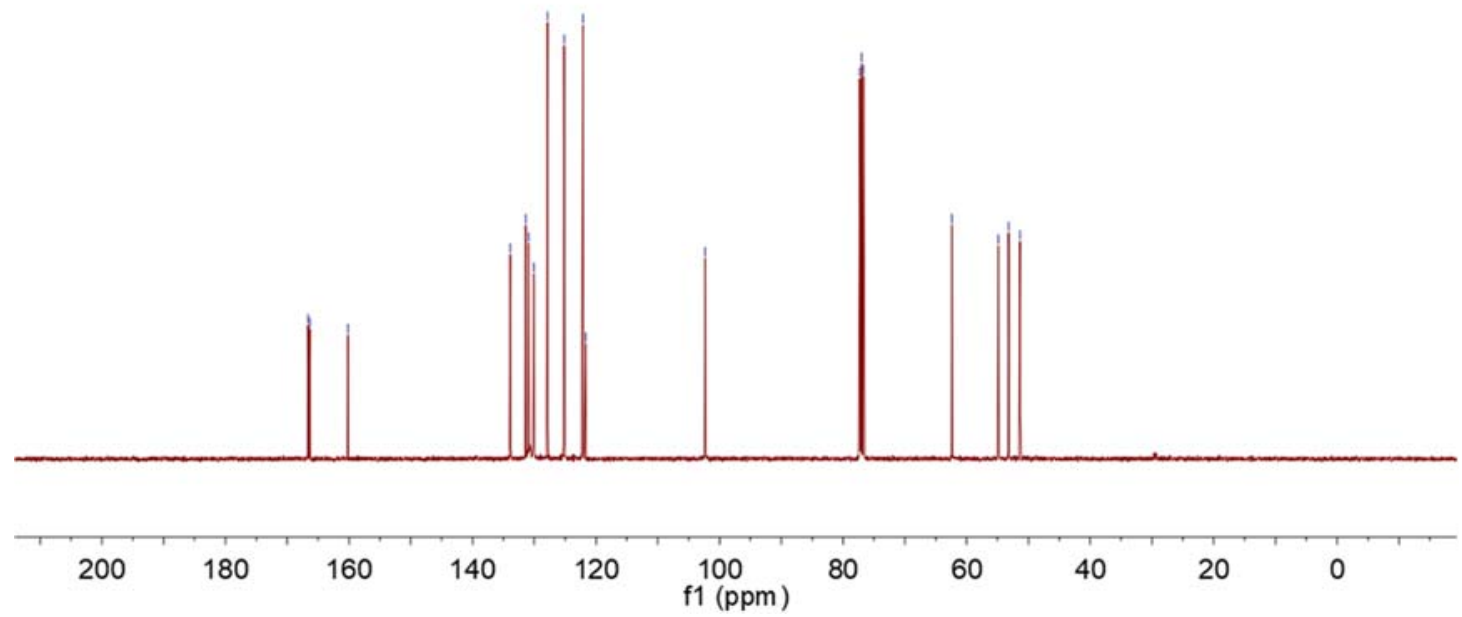


${ }^{1} \mathrm{H}$ NMR of $\mathbf{2 h}\left(400 \mathrm{MHz}, \mathrm{CDCl}_{3}\right)$

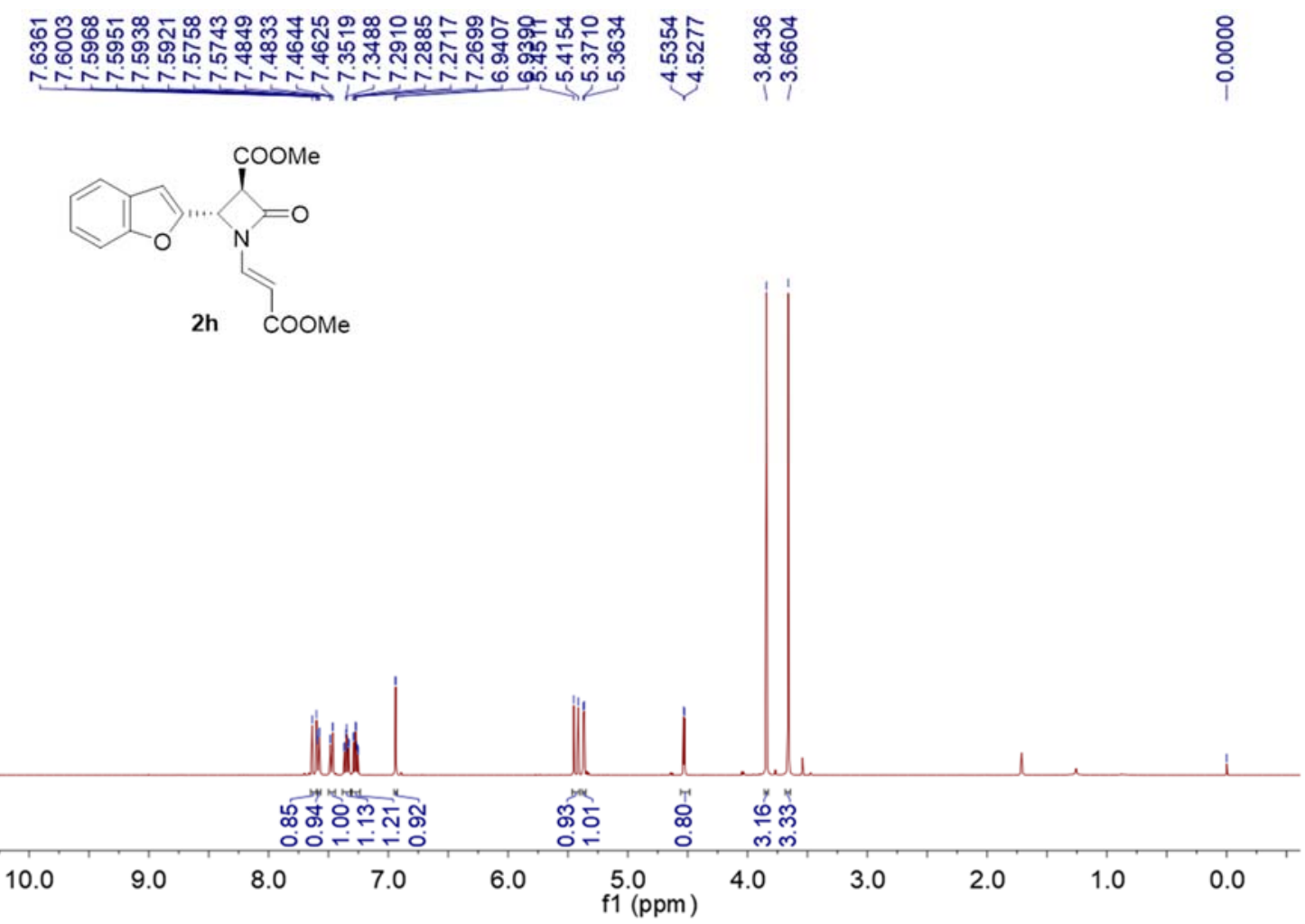

${ }^{13} \mathrm{C}$ NMR of $\mathbf{2 h}\left(100 \mathrm{MHz}, \mathrm{CDCl}_{3}\right)$
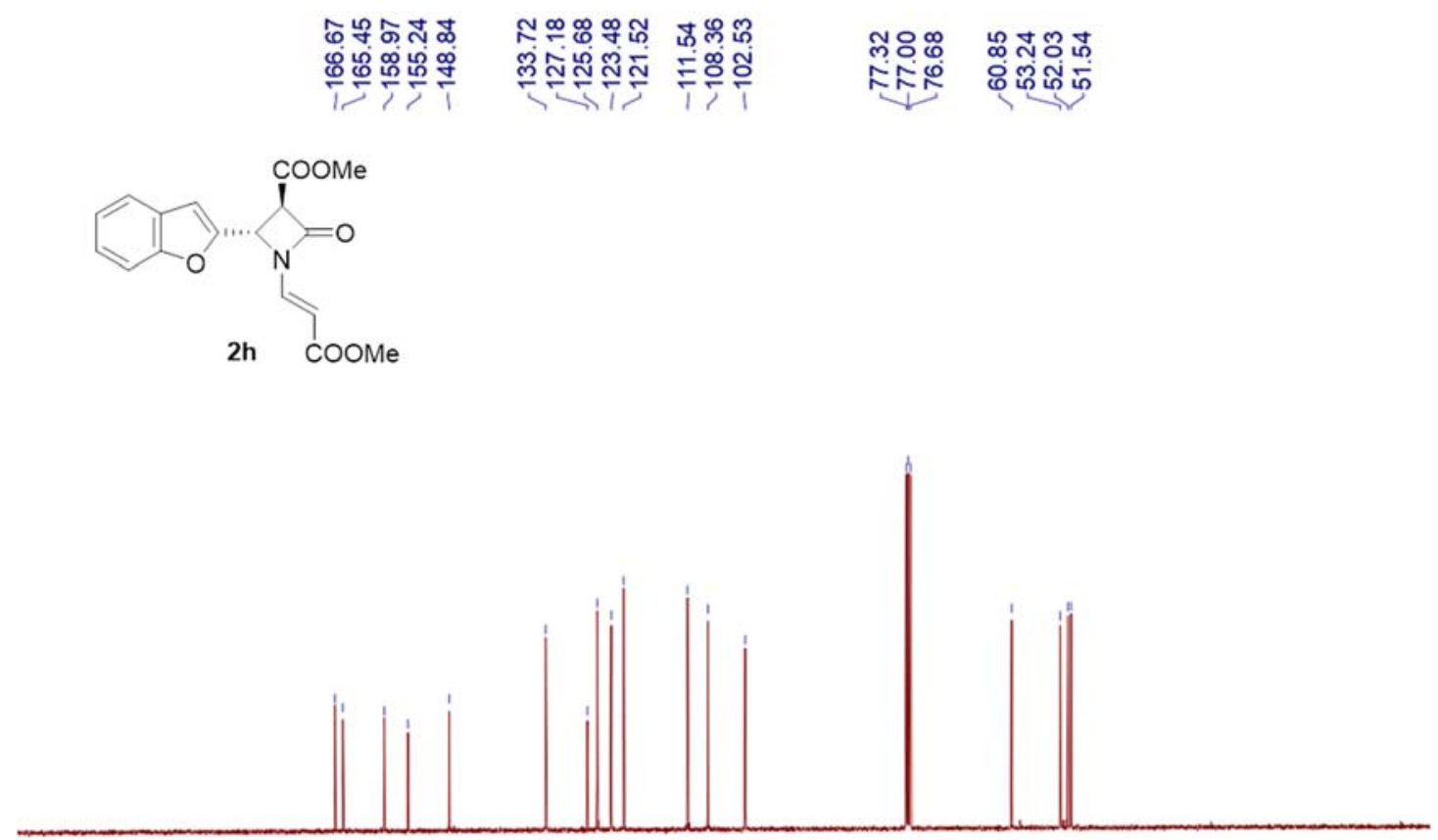

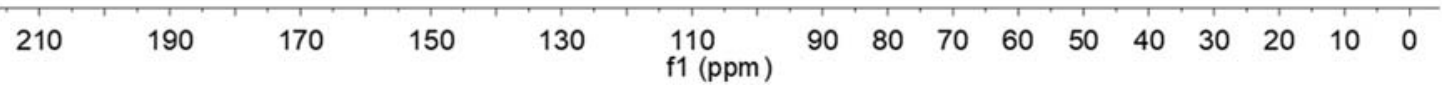


${ }^{1} \mathrm{H}$ NMR of $2 \mathbf{i}\left(400 \mathrm{MHz}, \mathrm{CDCl}_{3}\right)$

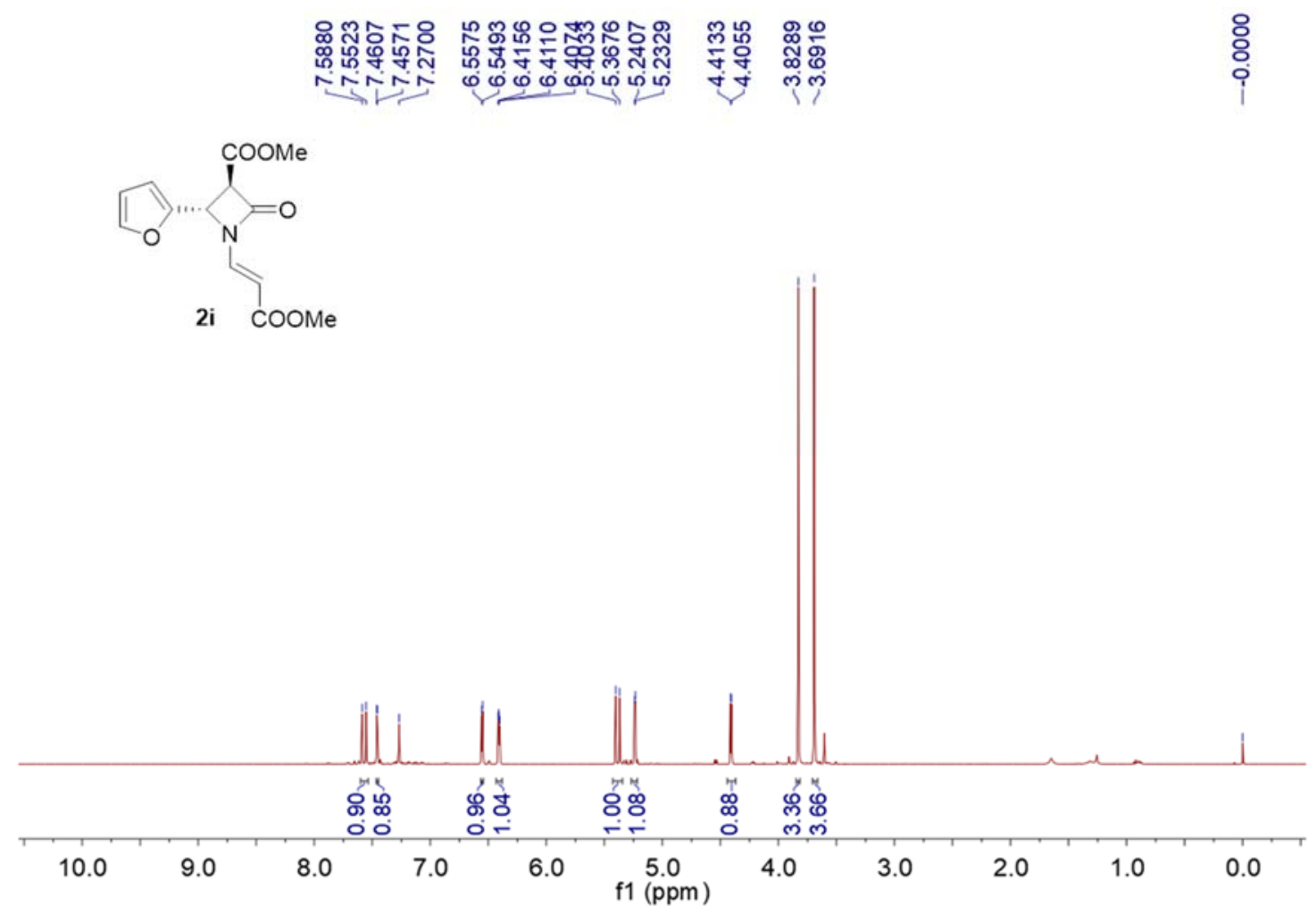

${ }^{13} \mathrm{C}$ NMR of $2 \mathbf{i}\left(100 \mathrm{MHz}, \mathrm{CDCl}_{3}\right)$
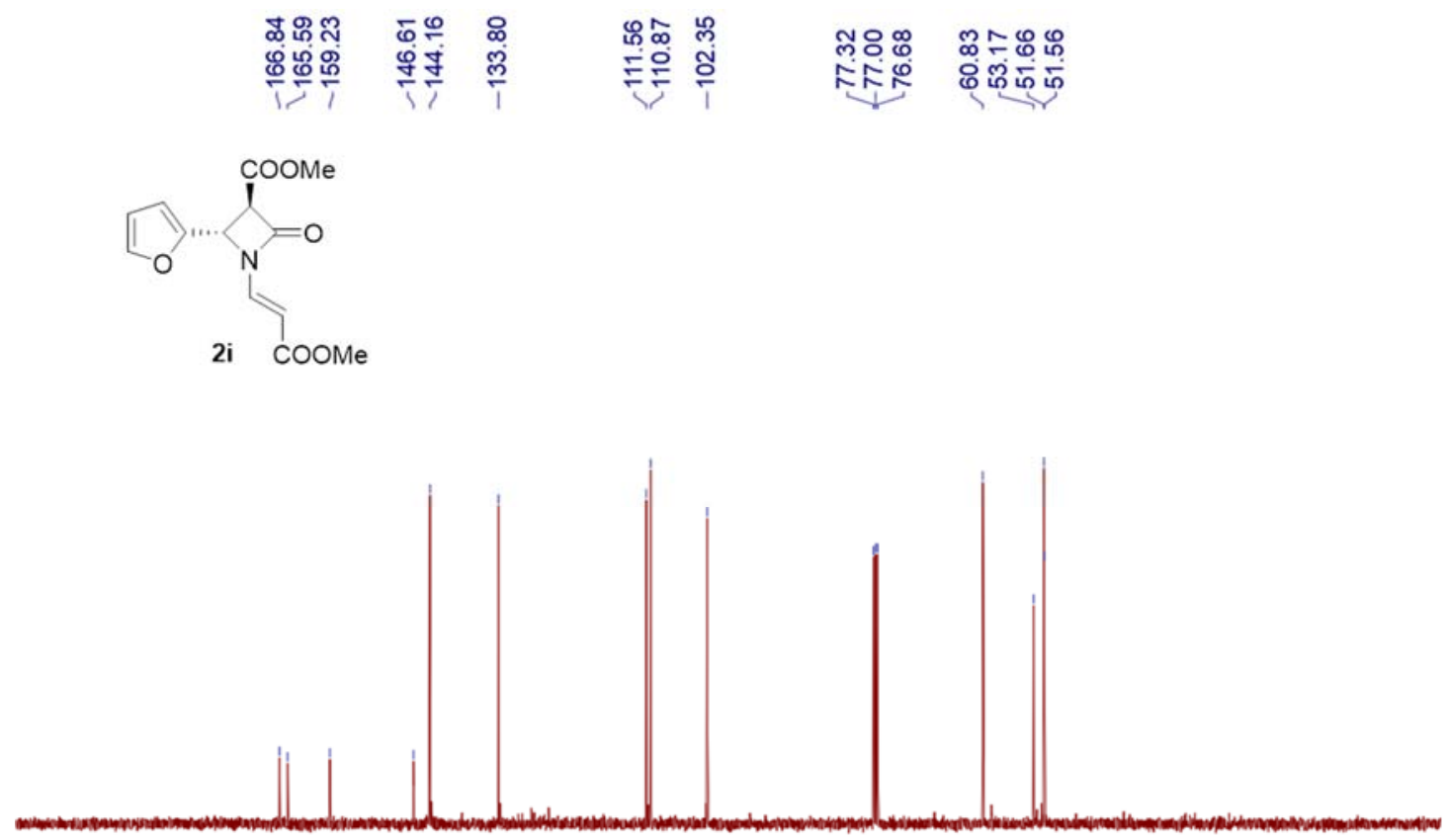

190

170

150

130

${ }_{\mathrm{f} 1}(\mathrm{ppm})$

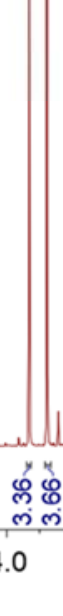


${ }^{1} \mathrm{H}$ NMR of $\mathbf{2} \mathbf{j}\left(400 \mathrm{MHz}, \mathrm{CDCl}_{3}\right)$

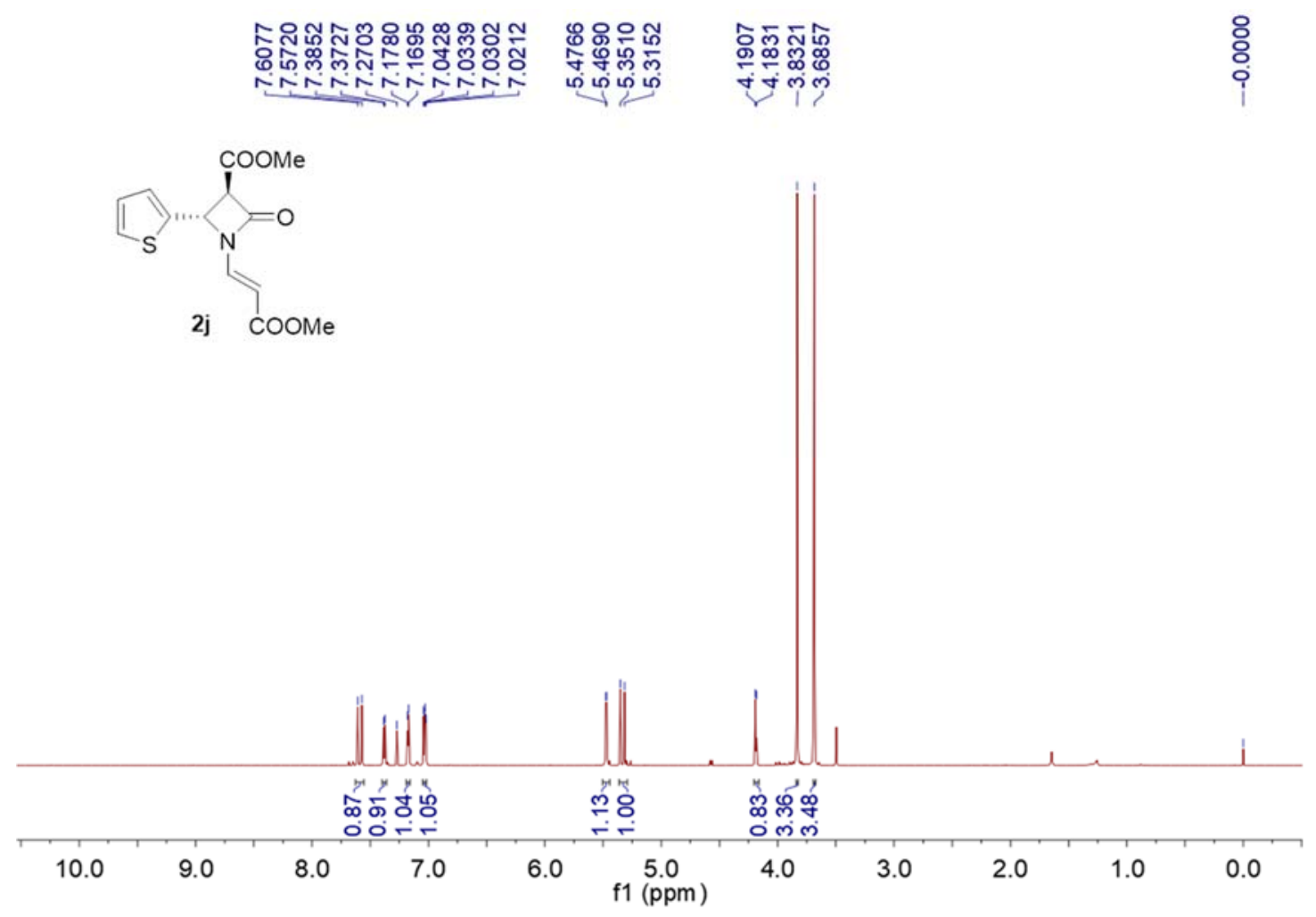

${ }^{13} \mathrm{C}$ NMR of $\mathbf{2 j}\left(100 \mathrm{MHz}, \mathrm{CDCl}_{3}\right)$

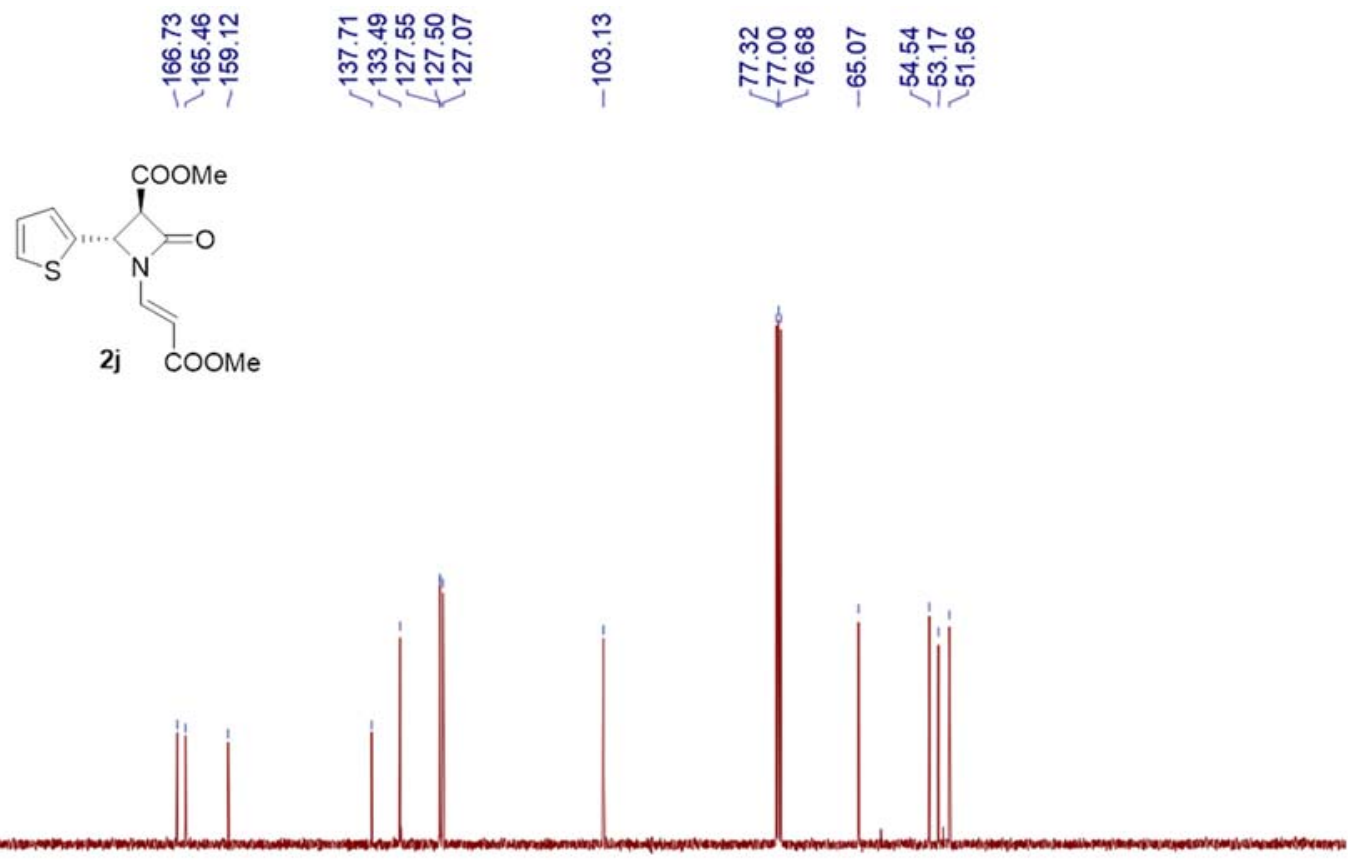

190

170

150

130

110 f1 (ppm)

$\begin{array}{lllllllll}80 & 70 & 60 & 50 & 40 & 30 & 20 & 10 & 0\end{array}$ 
${ }^{1} \mathrm{H}$ NMR of $\mathbf{2 k}\left(400 \mathrm{MHz}, \mathrm{CDCl}_{3}\right)$

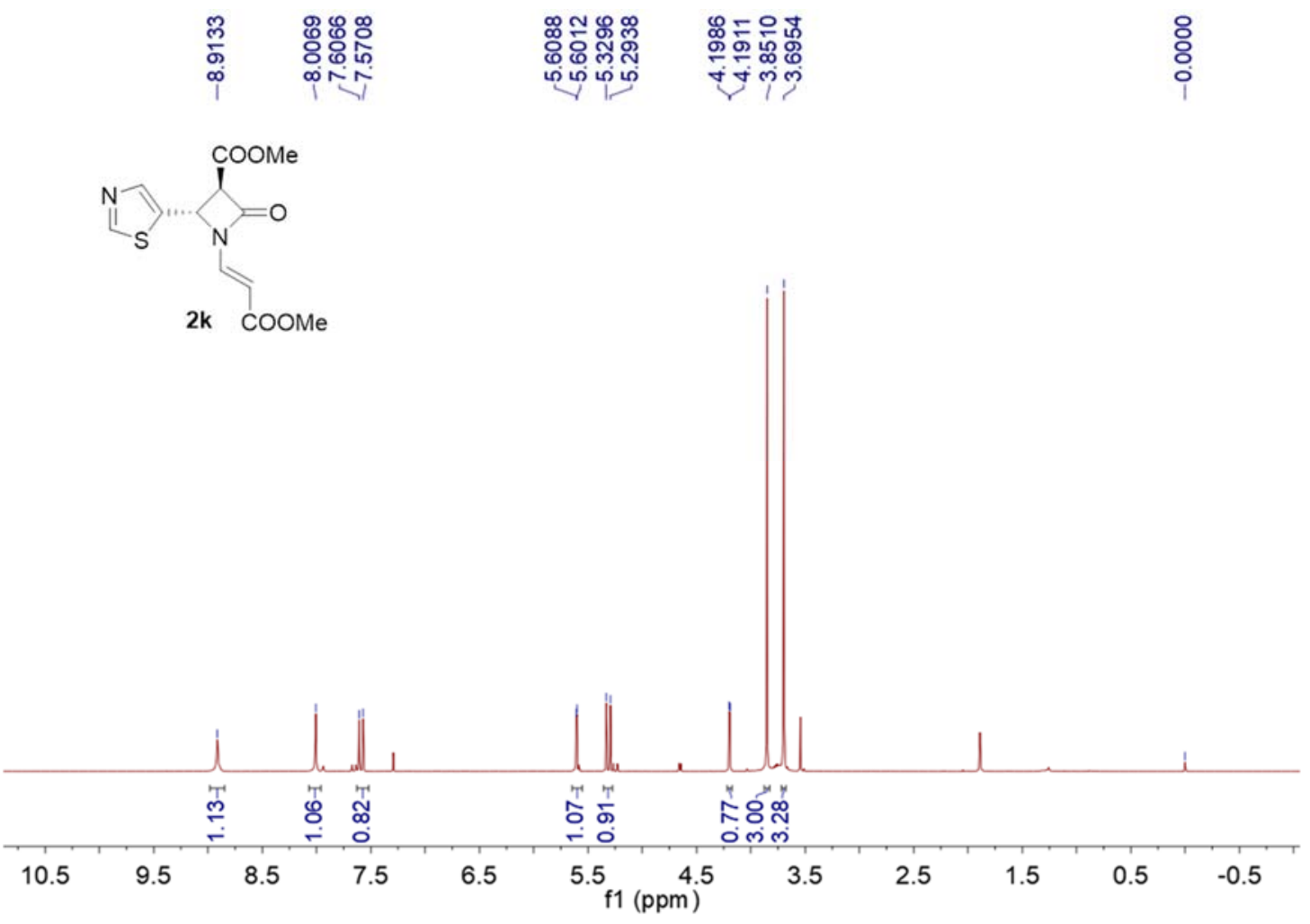

${ }^{13} \mathrm{C}$ NMR of $2 \mathbf{k}\left(100 \mathrm{MHz}, \mathrm{CDCl}_{3}\right)$
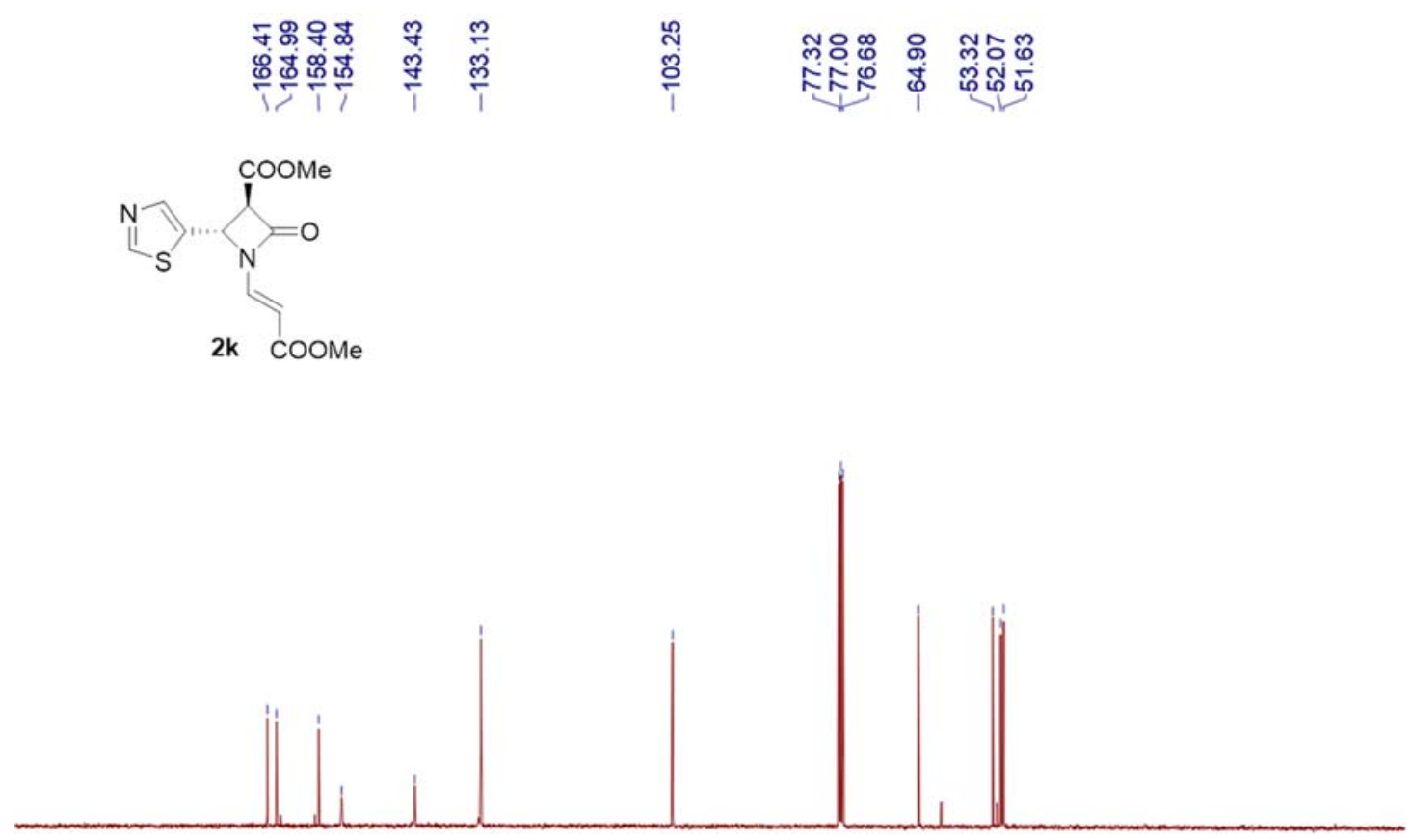

190

170

150

130

110 f1 (ppm)

$\begin{array}{lllllllll}80 & 70 & 60 & 50 & 40 & 30 & 20 & 10 & 0\end{array}$ 
${ }^{1} \mathrm{H}$ NMR of $21\left(400 \mathrm{MHz}, \mathrm{CDCl}_{3}\right)$

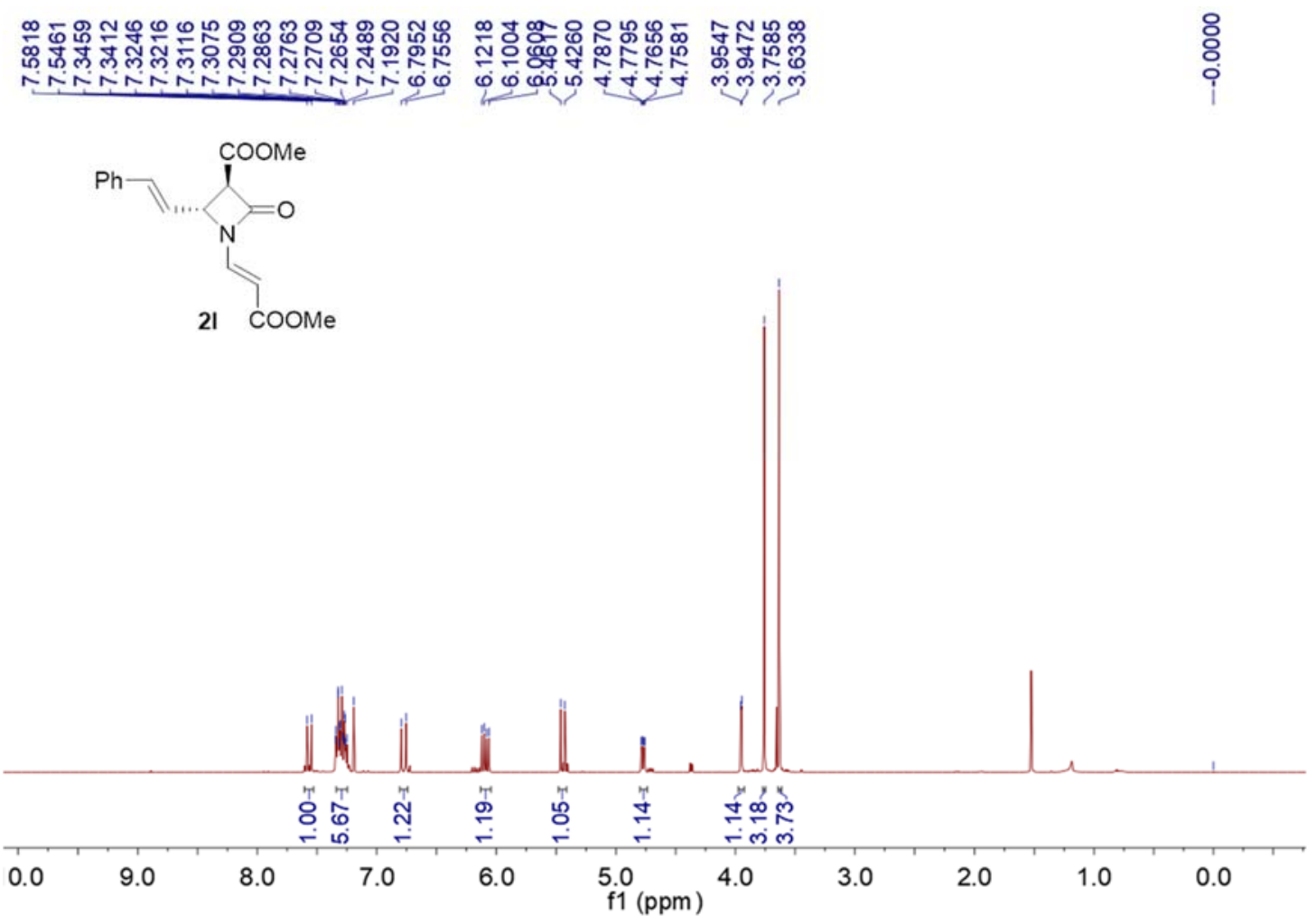

${ }^{13} \mathrm{C} \mathrm{NMR}$ of $2 \mathbf{l}\left(100 \mathrm{MHz}, \mathrm{CDCl}_{3}\right)$

\begin{tabular}{|c|c|c|c|}
\hline 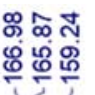 & 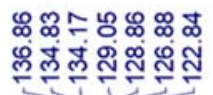 & $\begin{array}{l}\hat{0} \\
\text { ்ָ }\end{array}$ & 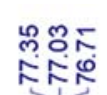 \\
\hline
\end{tabular}<smiles>COC(=O)/C=C/N1C(=O)[C@H](C(C)=O)[C@H]1/C=C/c1ccccc1</smiles>

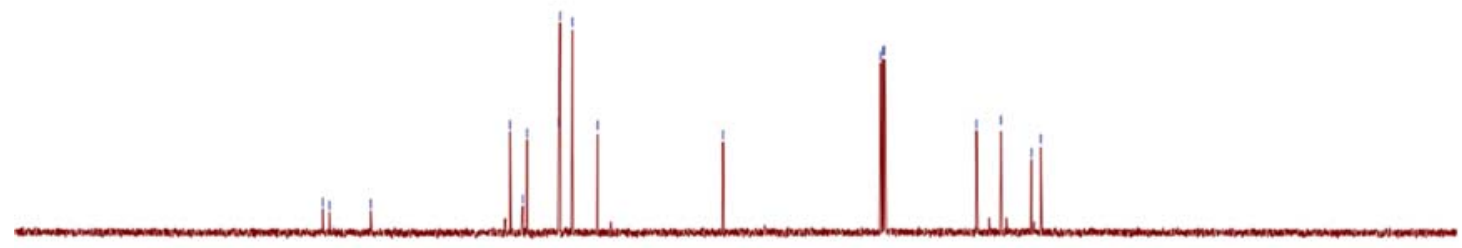

$200 \quad 180$

160

140

120 f1 (ppm)

80

60

40

$20 \quad 0$ 
${ }^{1} \mathrm{H}$ NMR of $\mathbf{2 m}\left(400 \mathrm{MHz}, \mathrm{CDCl}_{3}\right)$

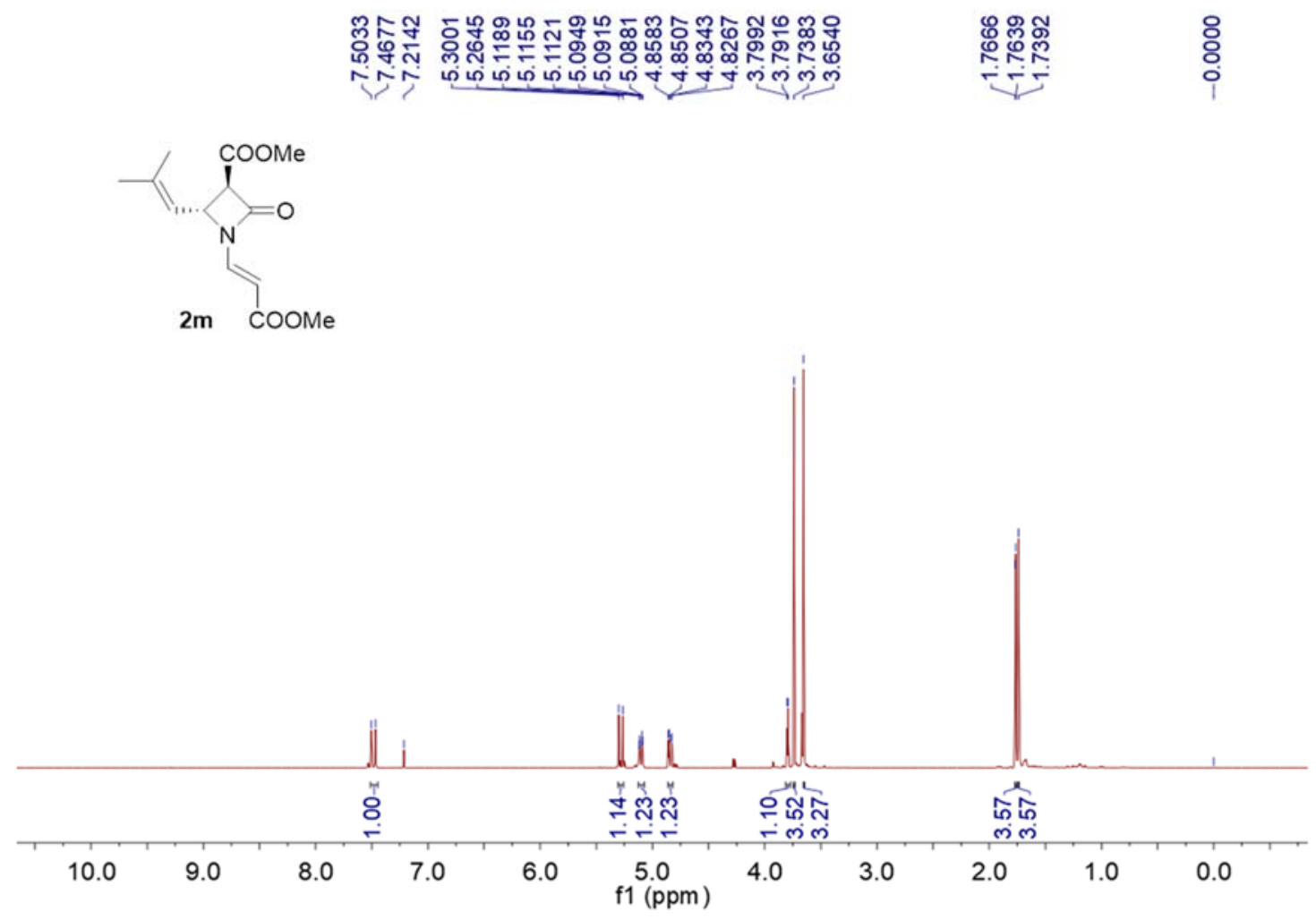

${ }^{13} \mathrm{C}$ NMR of $\mathbf{2 m}\left(100 \mathrm{MHz}, \mathrm{CDCl}_{3}\right)$
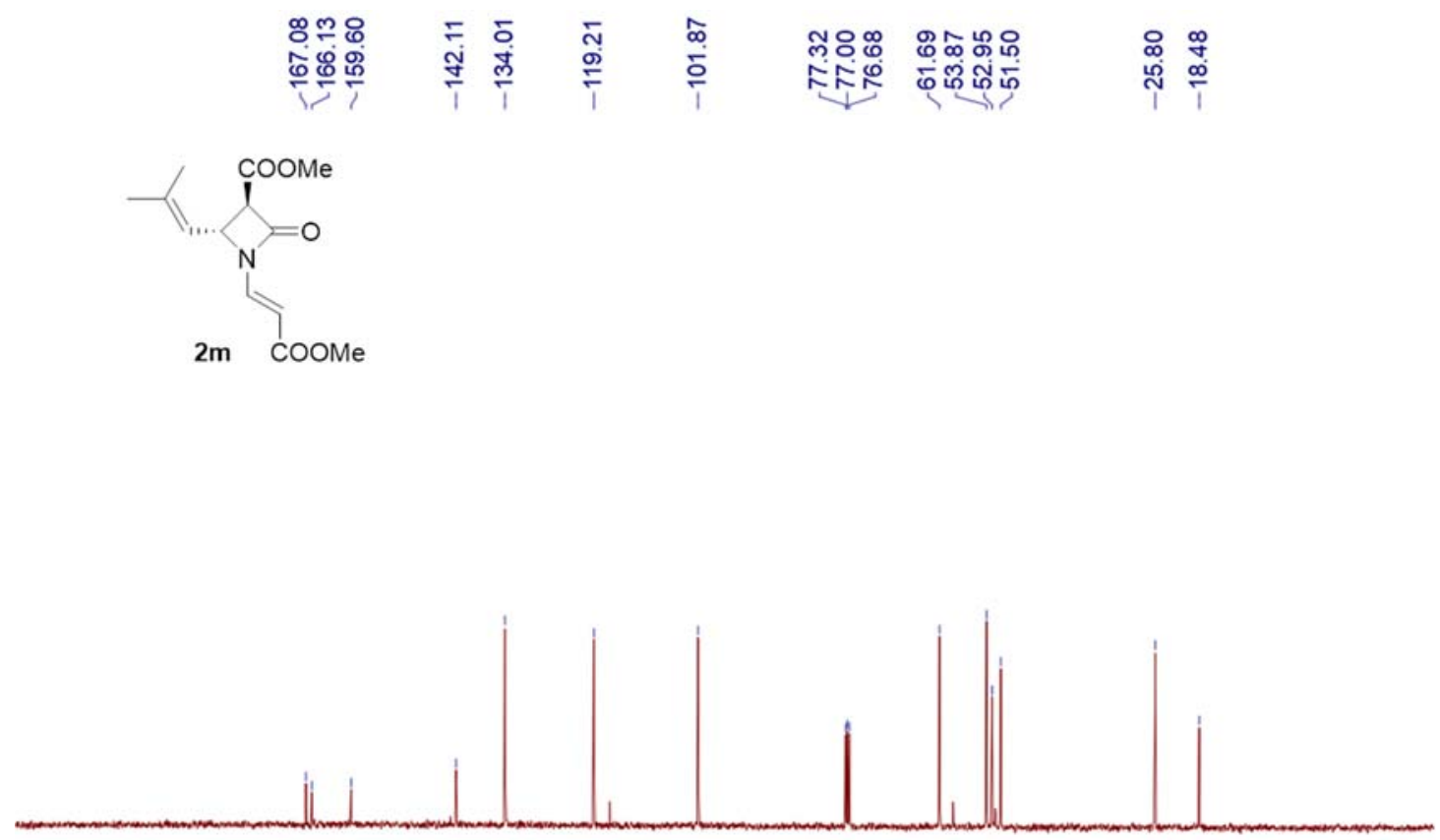

200

$180 \quad 160$

140

$120 \quad 100$

80

60

40

20

$\begin{array}{ll}0 & -10\end{array}$ 
${ }^{1} \mathrm{H} \mathrm{NMR}$ of $2 \mathbf{n}\left(400 \mathrm{MHz}, \mathrm{CDCl}_{3}\right)$

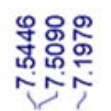

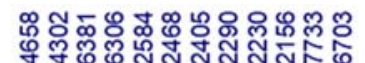<smiles>CCOC(=O)C(C(C)=O)N(C=CC(C)=O)C(C)=O</smiles>

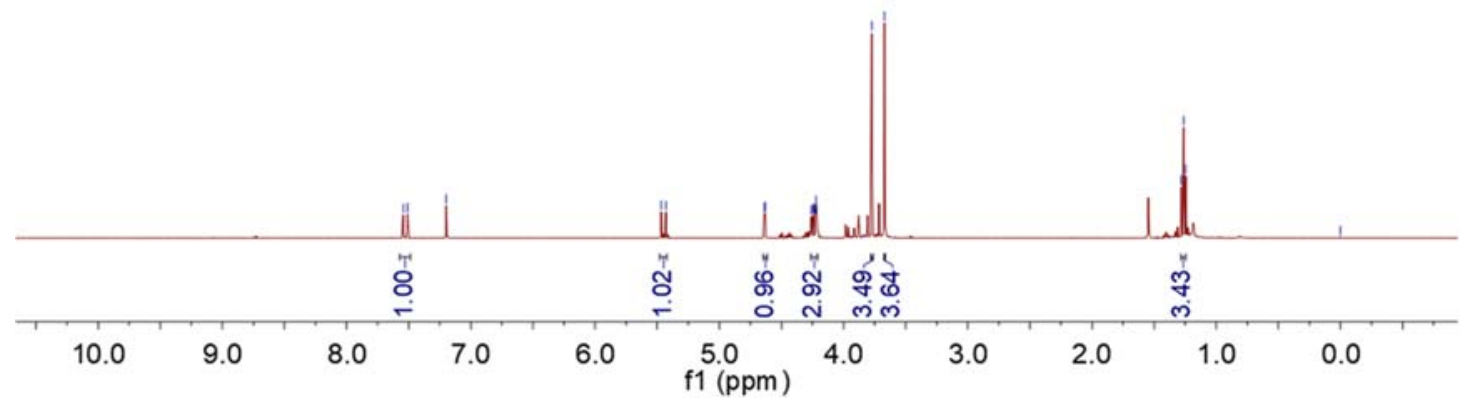

${ }^{13} \mathrm{C}$ NMR of $2 \mathbf{n}\left(100 \mathrm{MHz}, \mathrm{CDCl}_{3}\right)$
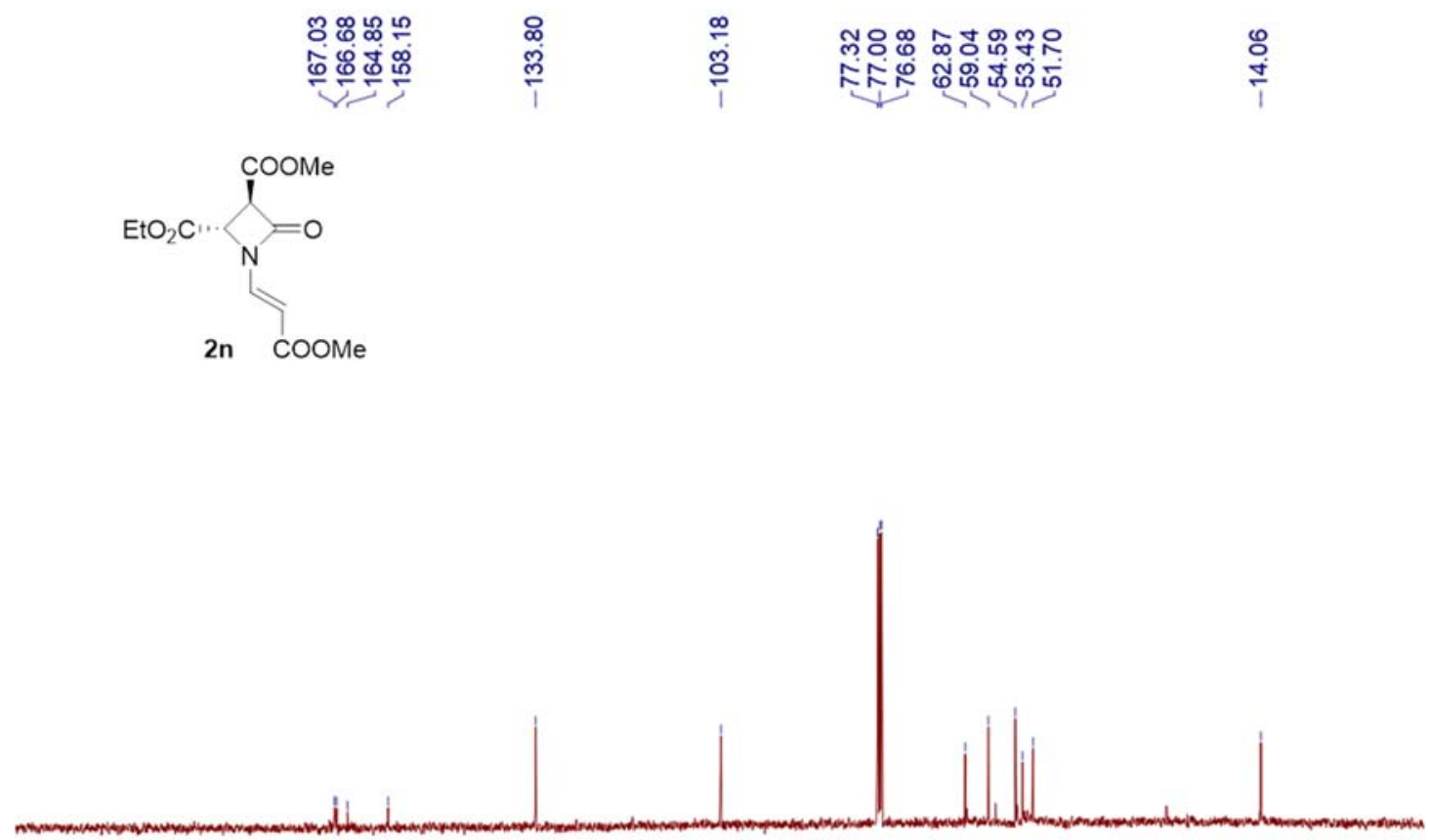

200

180

160

140

120 f1 $(\mathrm{ppm}$

80

60

40

20

0 
${ }^{1} \mathrm{H}$ NMR of $2 \mathbf{o}\left(400 \mathrm{MHz}, \mathrm{CDCl}_{3}\right)$

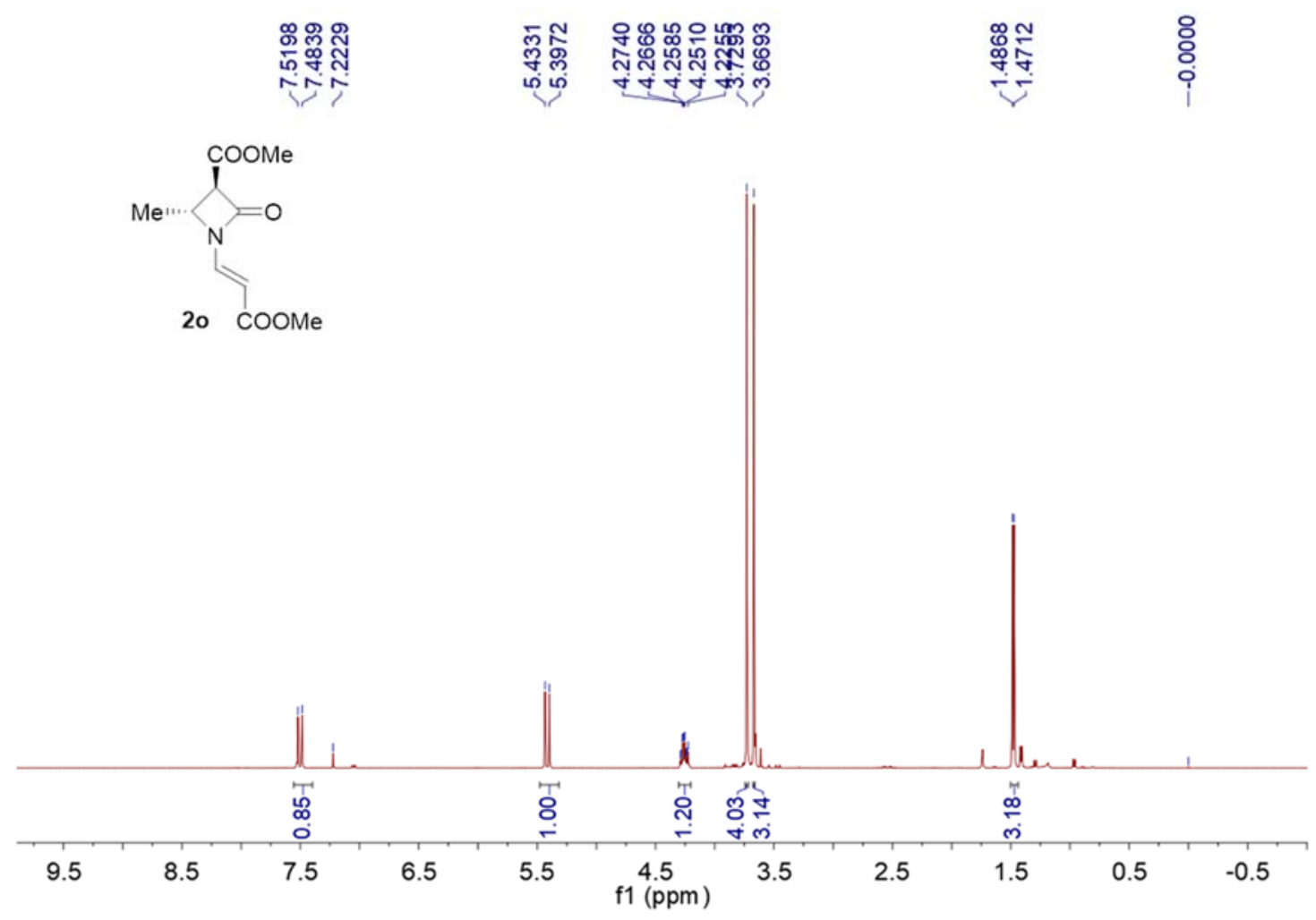

${ }^{13} \mathrm{C}$ NMR of $2 \mathrm{o}\left(100 \mathrm{MHz}, \mathrm{CDCl}_{3}\right)$
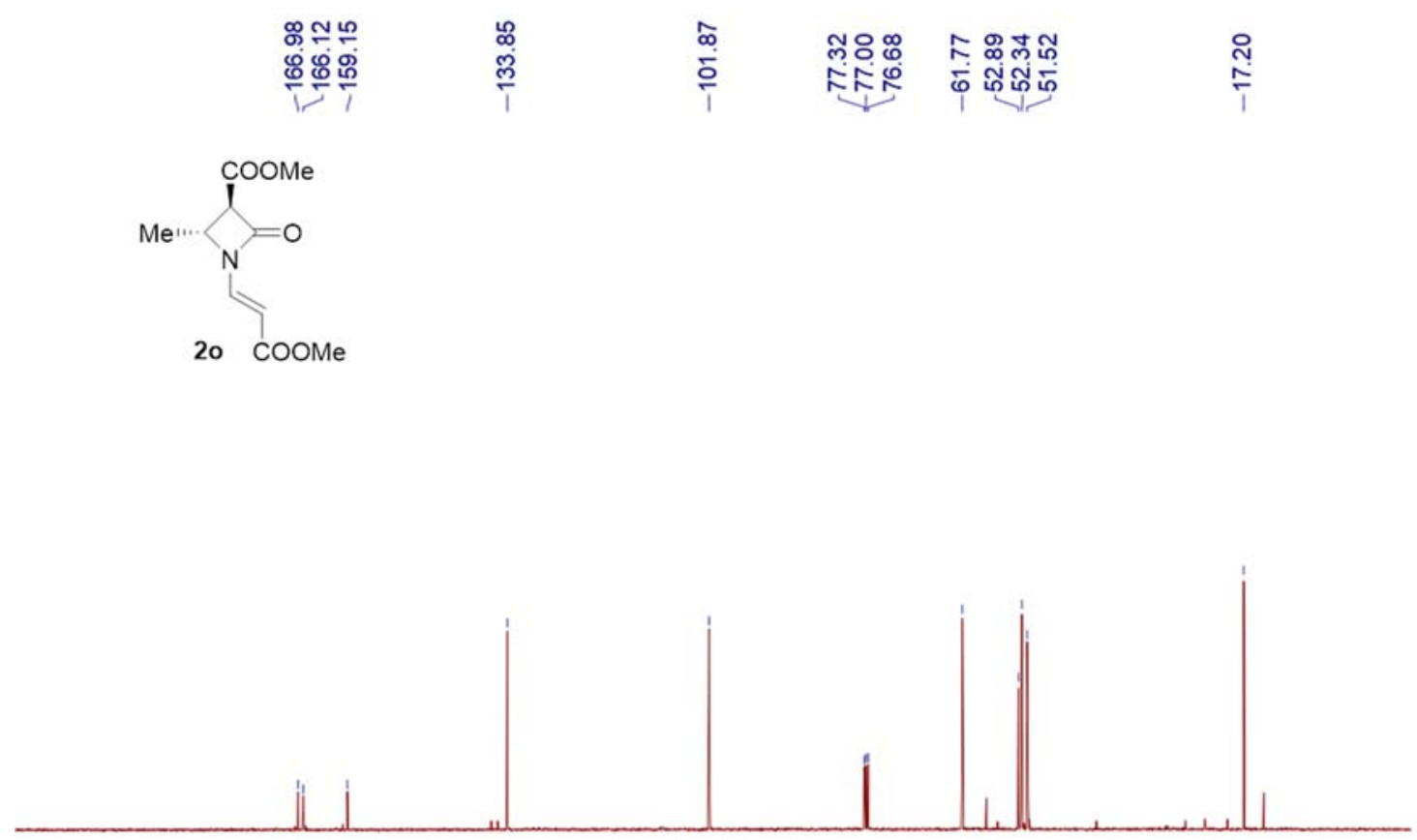

\begin{tabular}{llllllllllllllll}
\hline 10 & 190 & 170 & 150 & 130 & 110 & 90 & 80 & 70 & 60 & 50 & 40 & 30 & 20 & 10 & 0
\end{tabular} 
${ }^{1} \mathrm{H}$ NMR of $2 \mathbf{p}\left(400 \mathrm{MHz}, \mathrm{CDCl}_{3}\right)$

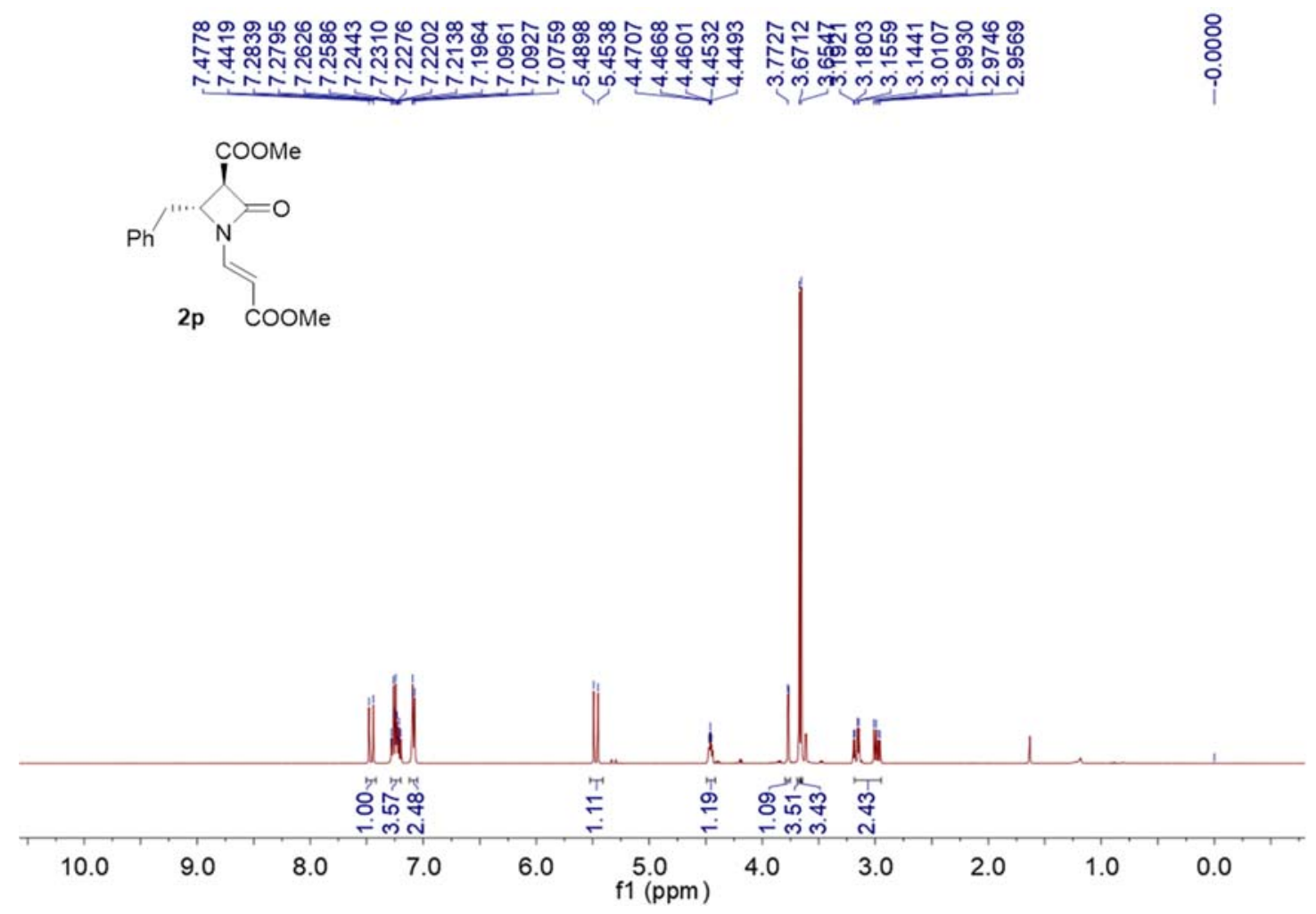

${ }^{13} \mathrm{C}$ NMR of $2 \mathbf{p}\left(100 \mathrm{MHz}, \mathrm{CDCl}_{3}\right)$

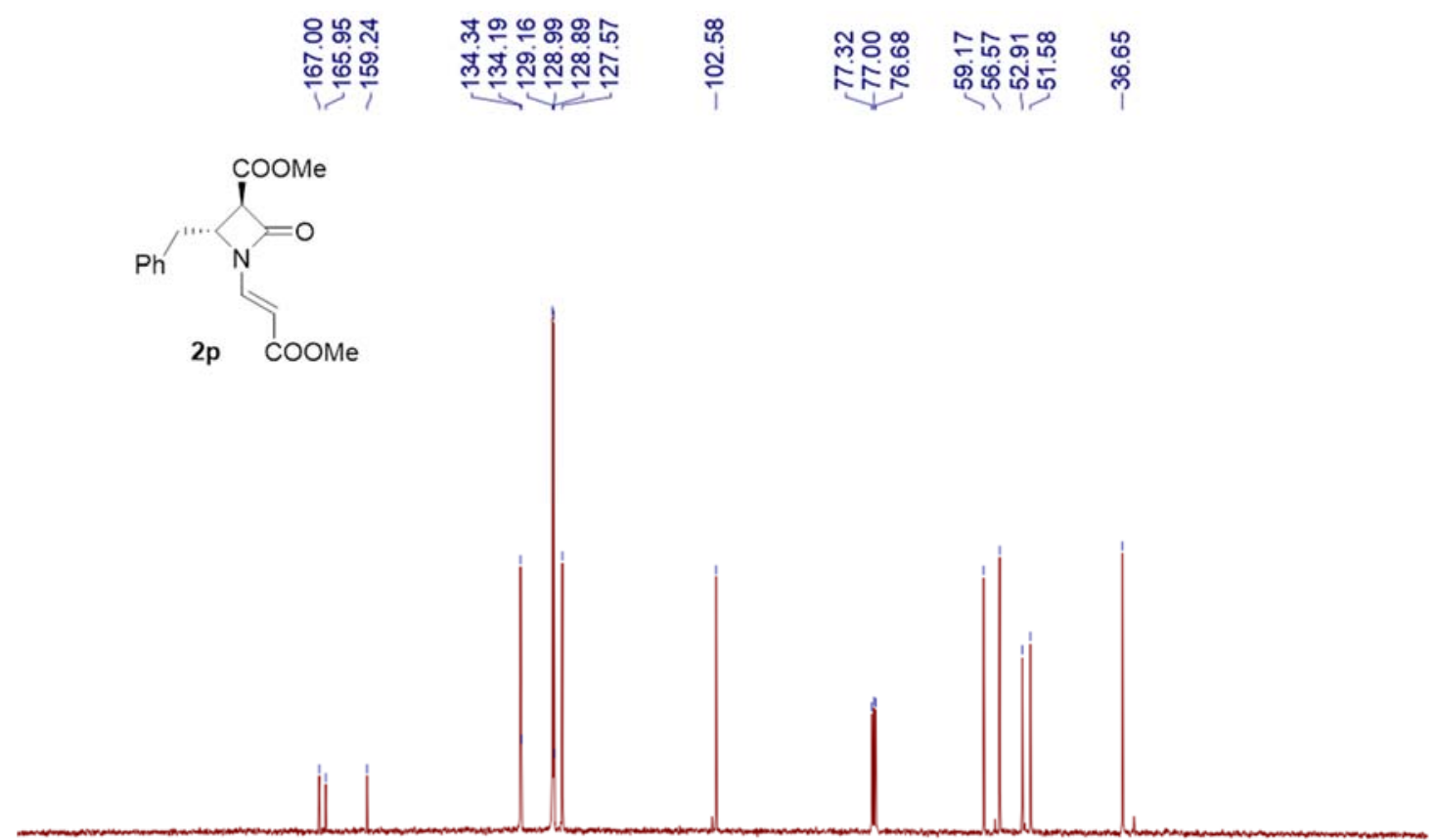

\begin{tabular}{llllllllllllllll}
\hline 210 & 190 & 170 & 150 & 130 & 110 & 90 & 80 & 70 & 60 & 50 & 40 & 30 & 20 & 10 & 0
\end{tabular}


${ }^{1} \mathrm{H}$ NMR of $2 q\left(400 \mathrm{MHz}, \mathrm{CDCl}_{3}\right)$

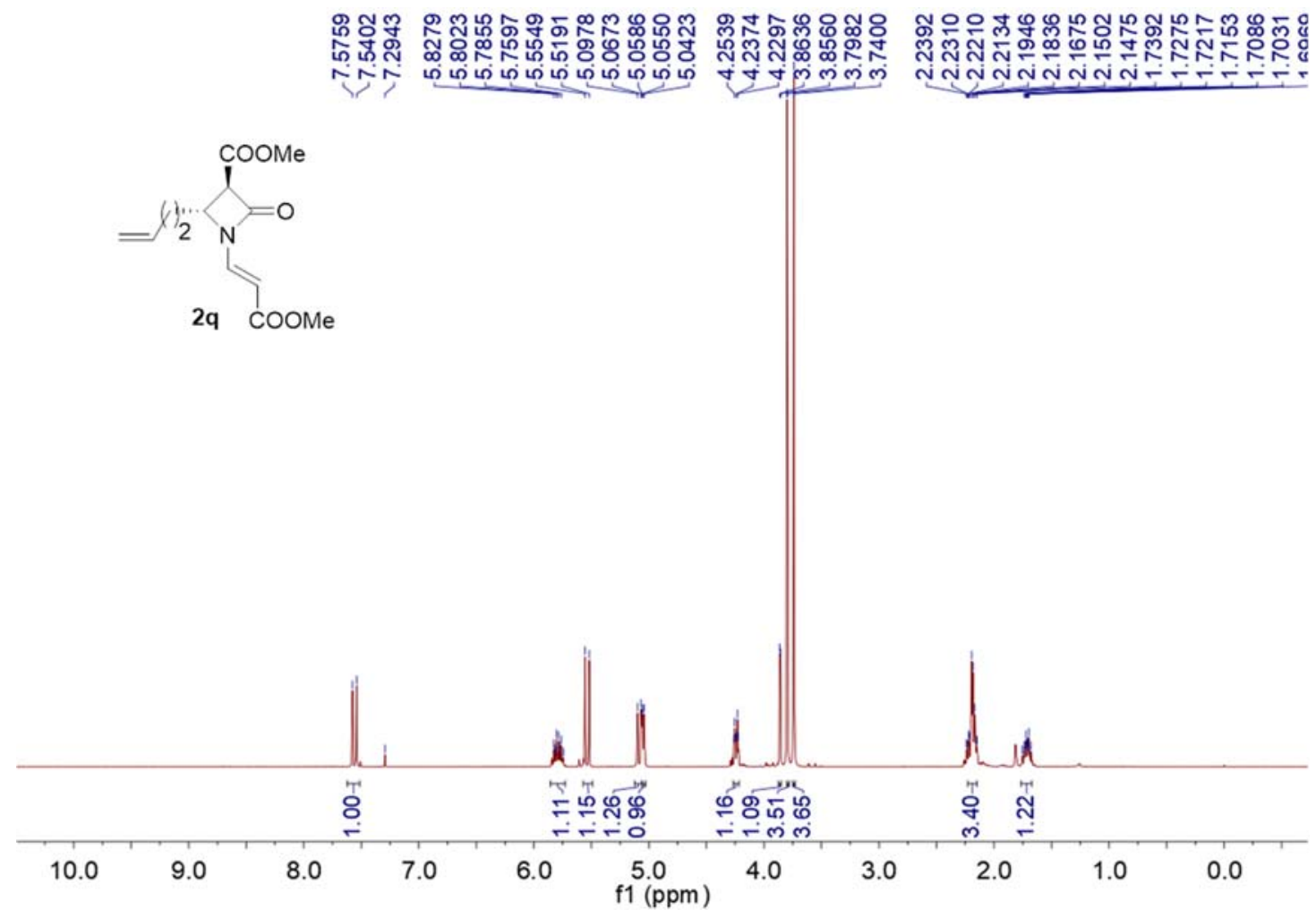

${ }^{13} \mathrm{C}$ NMR of $2 q\left(100 \mathrm{MHz}, \mathrm{CDCl}_{3}\right)$
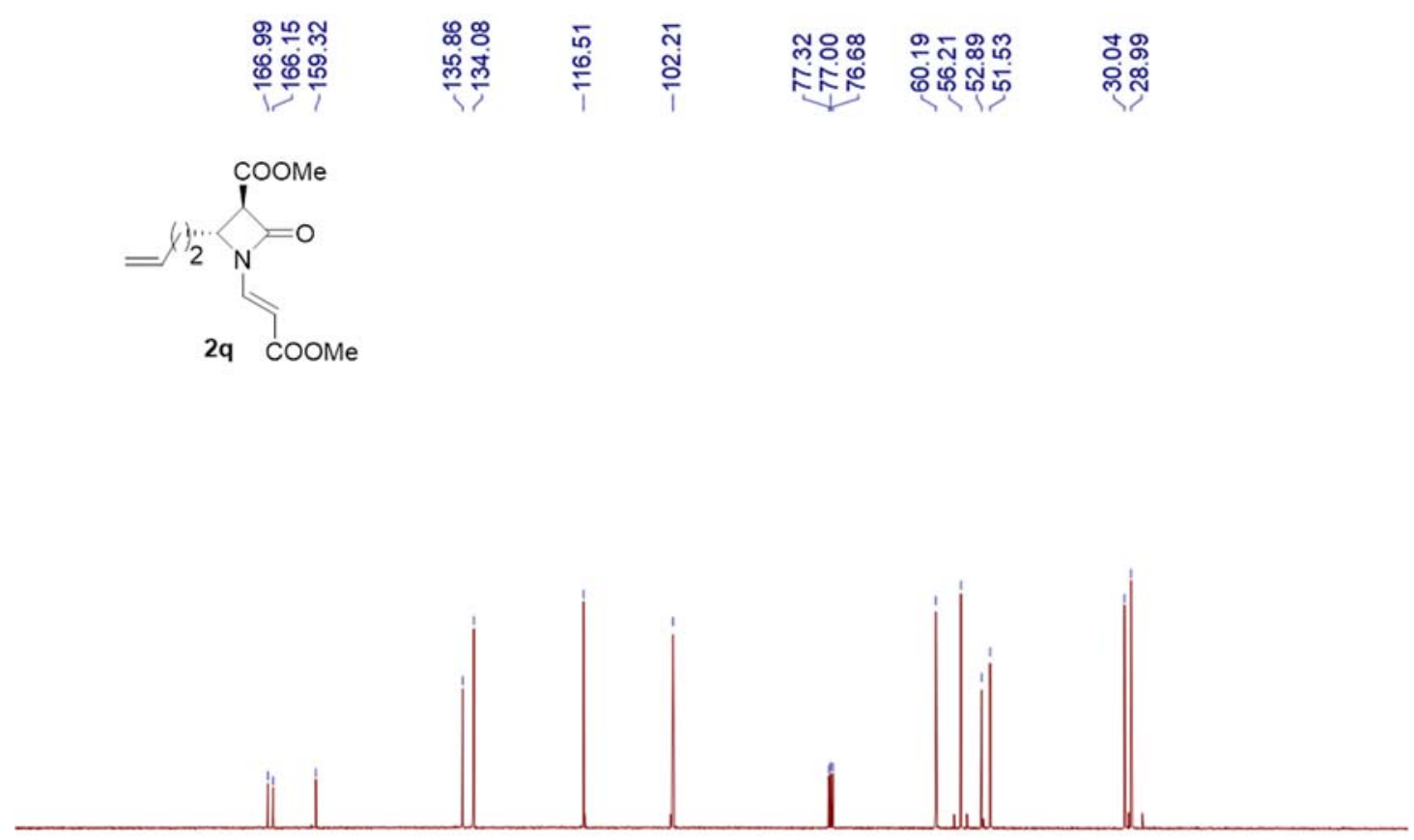

$190170 \quad 150 \quad 130 \quad 110^{\mathrm{f} 1(\mathrm{ppm})} \begin{array}{rlllllllllll}90 & 80 & 70 & 60 & 50 & 40 & 30 & 20 & 10 & 0\end{array}$ 
${ }^{1} \mathrm{H}$ NMR of $2 \mathbf{r}\left(400 \mathrm{MHz}, \mathrm{CDCl}_{3}\right)$

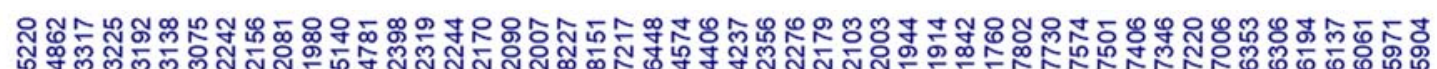

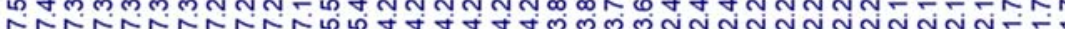
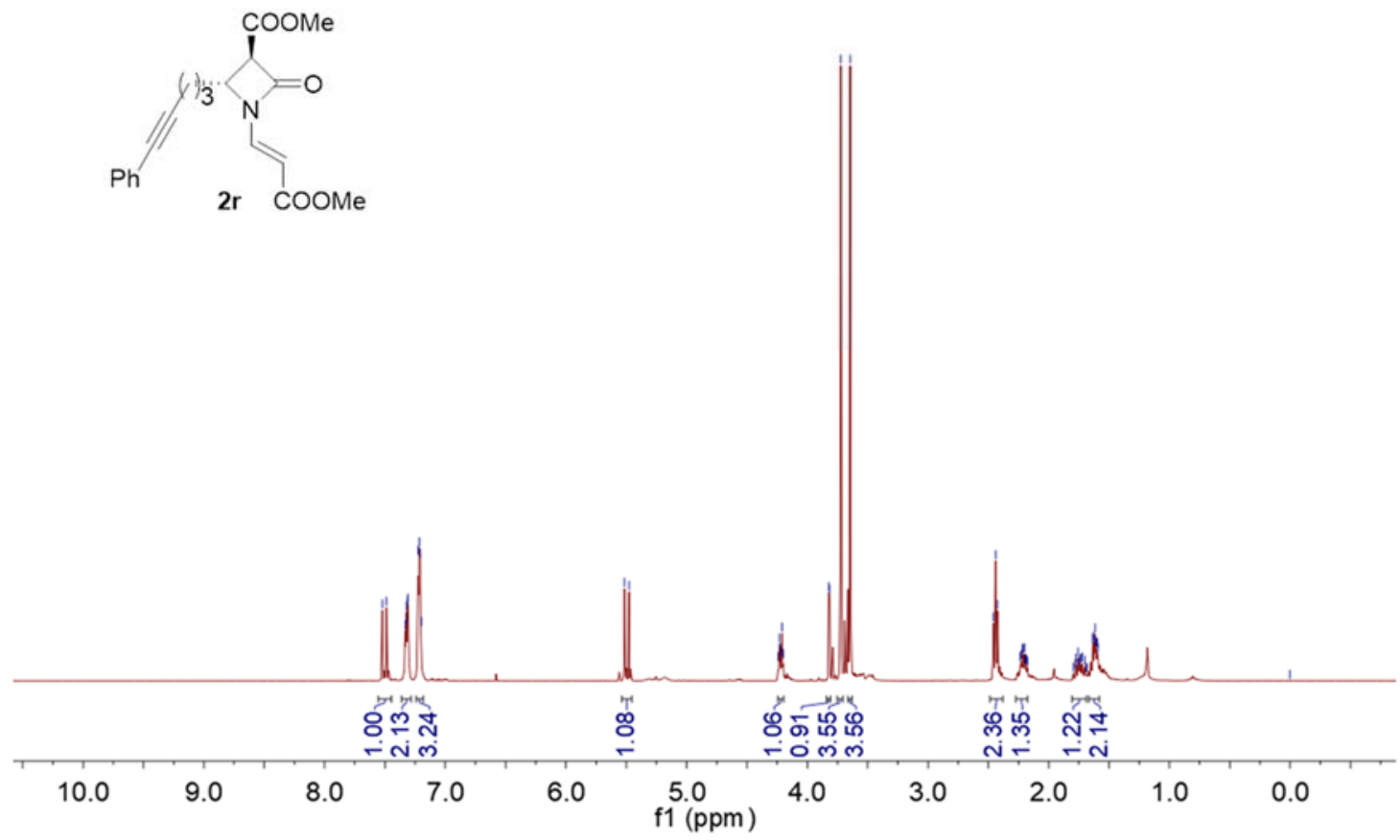

${ }^{13} \mathrm{C}$ NMR of $2 \mathbf{r}\left(100 \mathrm{MHz}, \mathrm{CDCl}_{3}\right)$
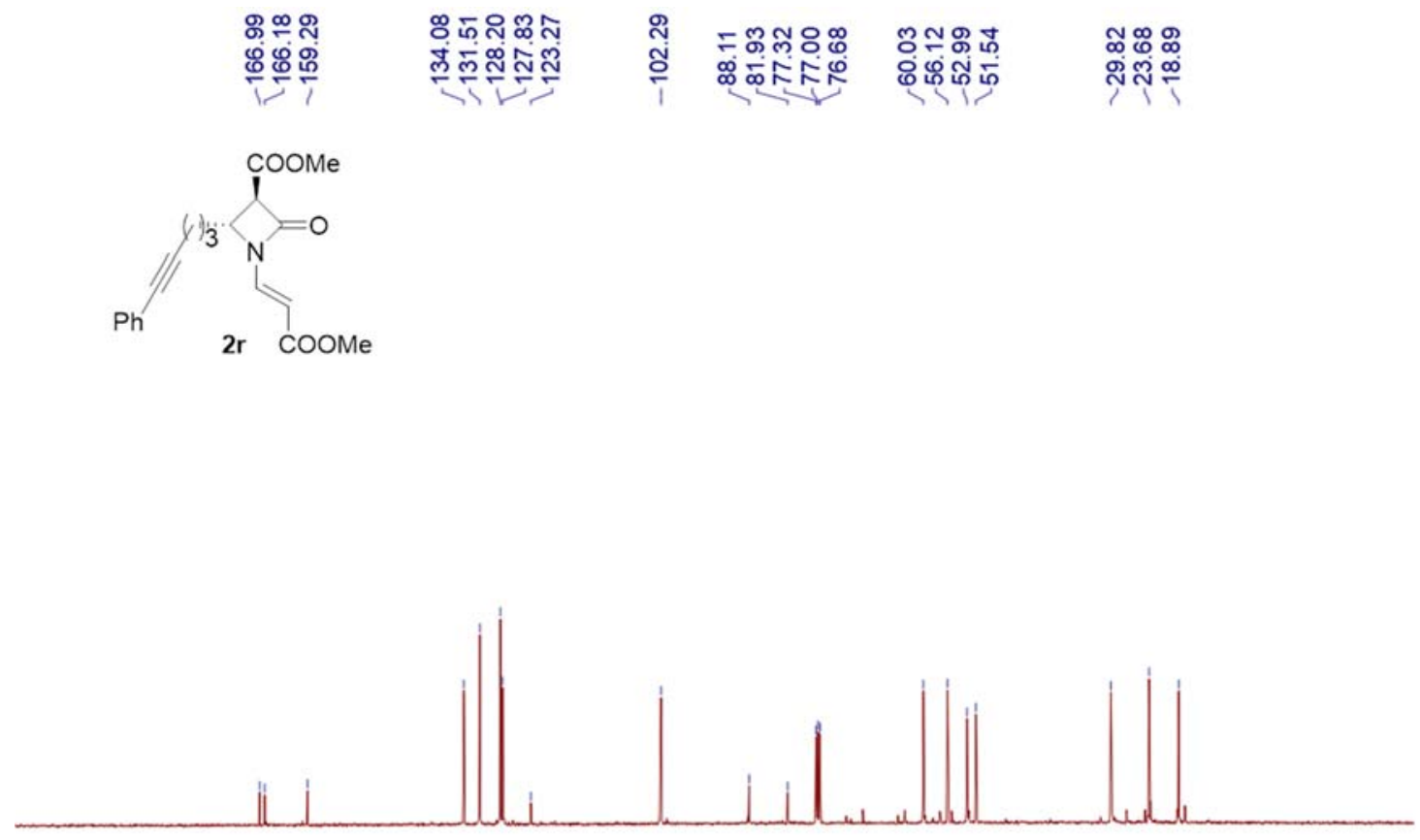

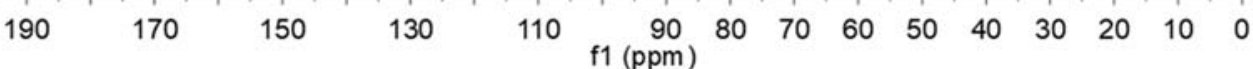


${ }^{1} \mathrm{H}$ NMR of $2 \mathbf{s}\left(400 \mathrm{MHz}, \mathrm{CDCl}_{3}\right)$

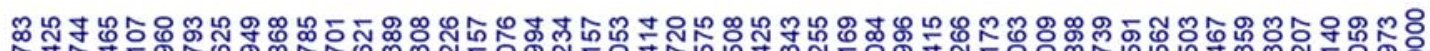

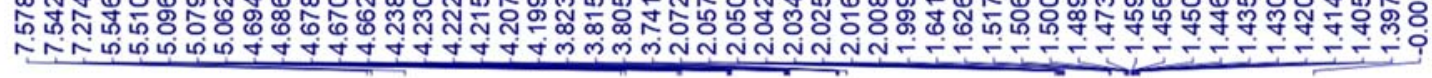
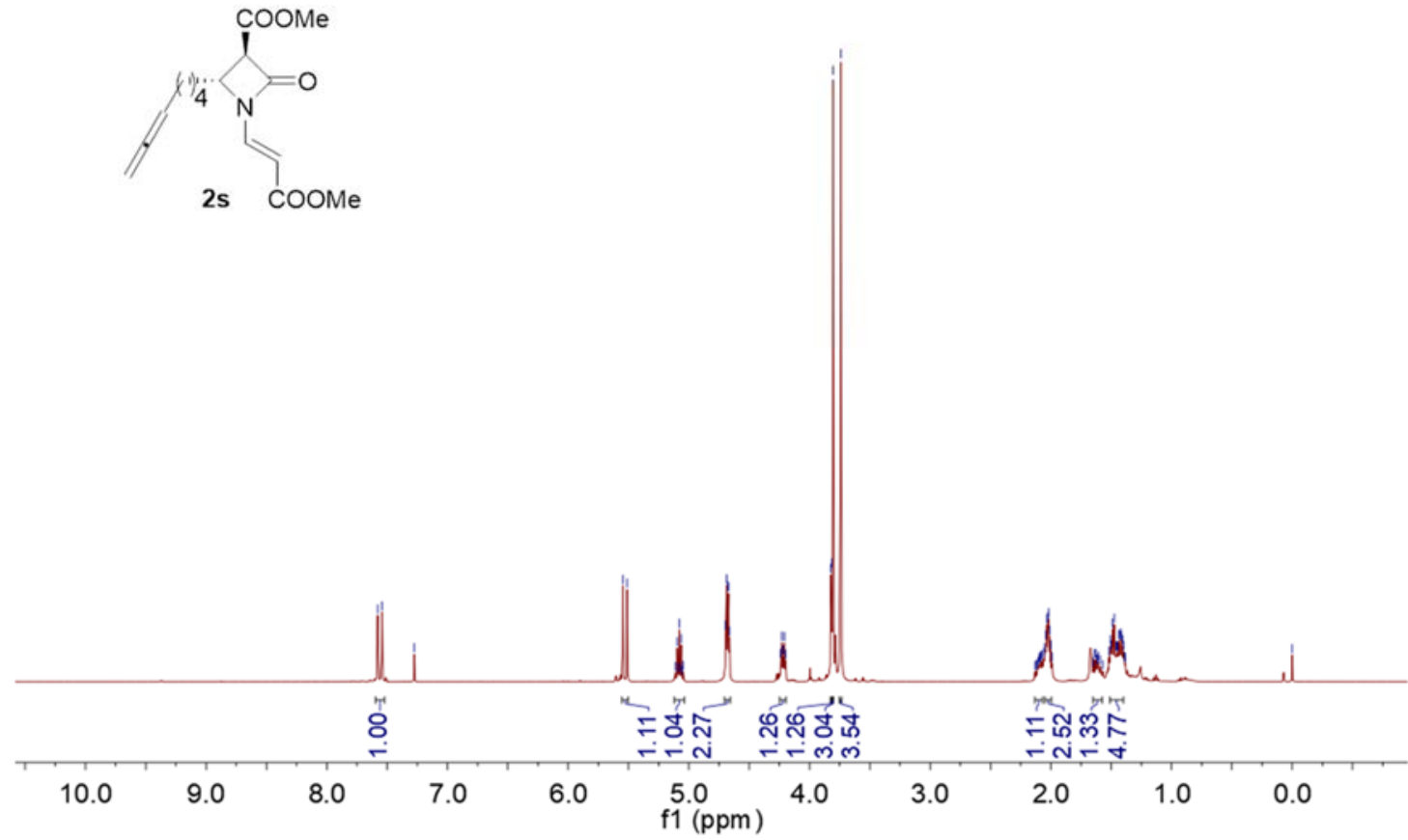

${ }^{13} \mathrm{C}$ NMR of $2 \mathbf{s}\left(100 \mathrm{MHz}, \mathrm{CDCl}_{3}\right)$
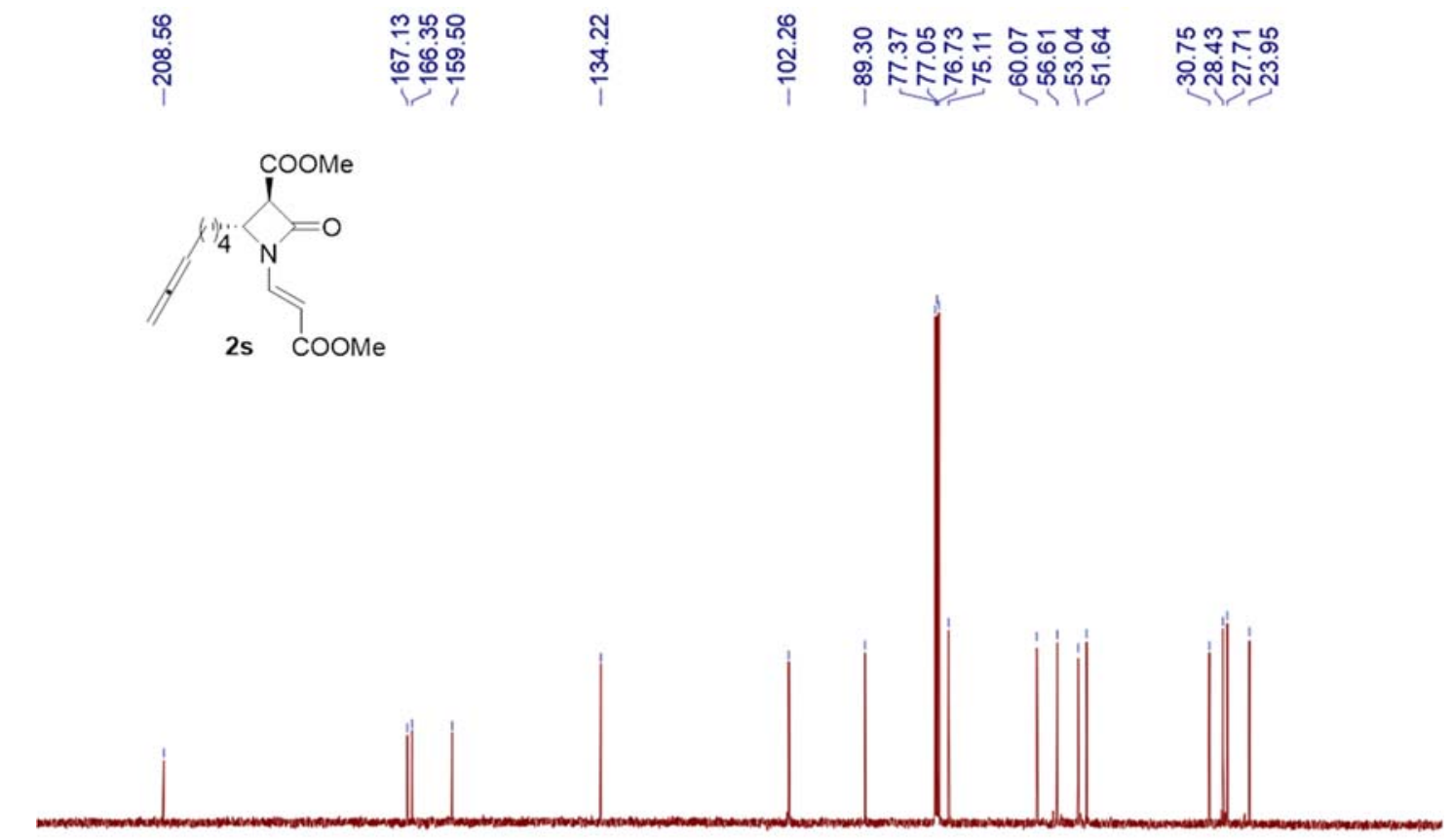

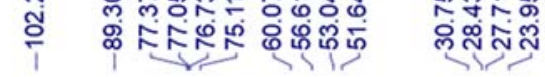

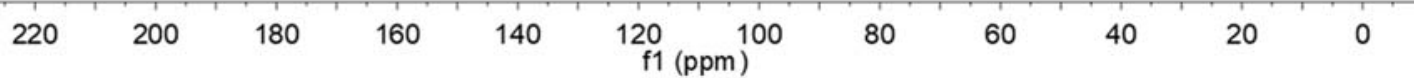


${ }^{1} \mathrm{H}$ NMR of $\mathbf{2 t}\left(400 \mathrm{MHz}, \mathrm{CDCl}_{3}\right)$
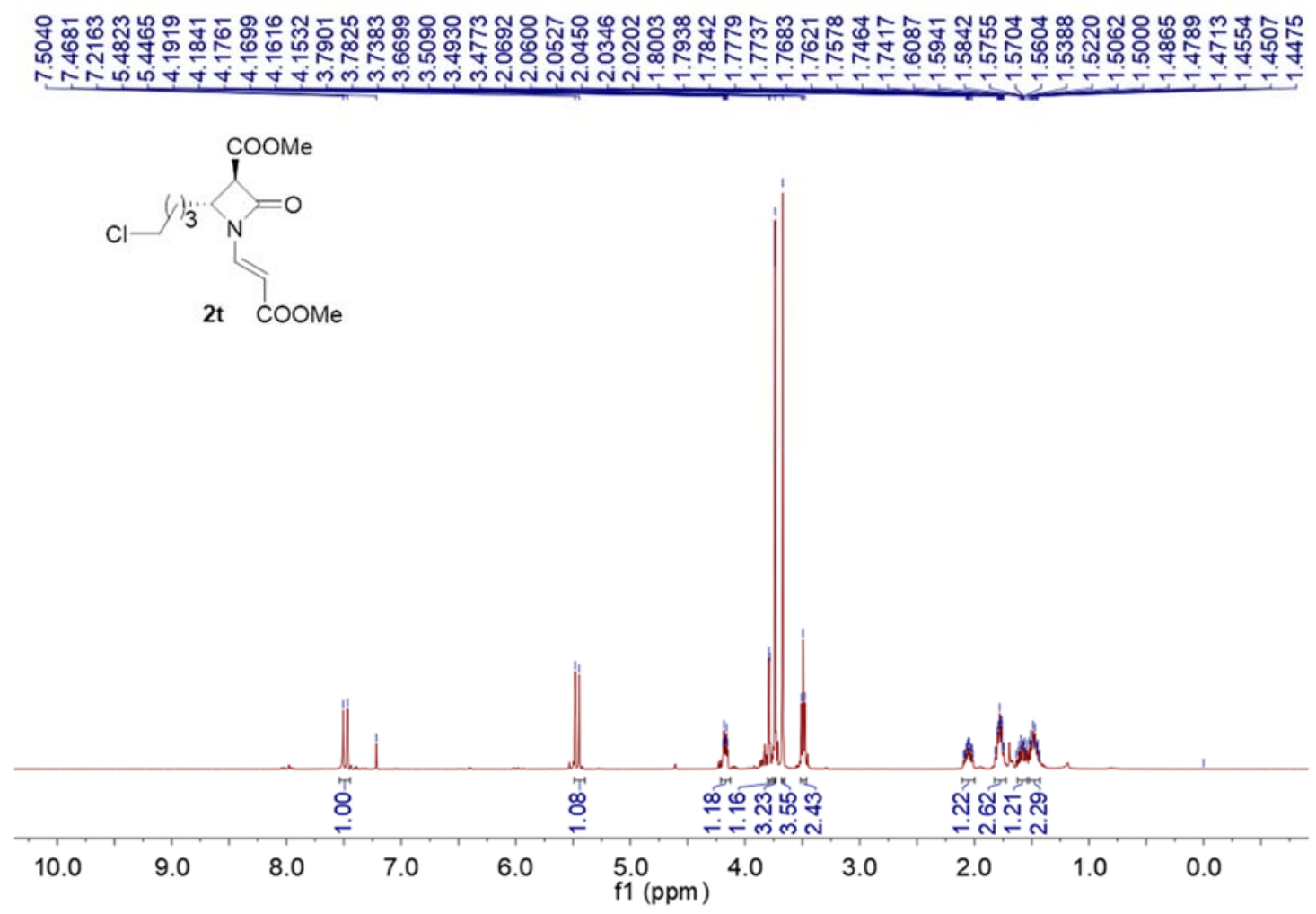

${ }^{13} \mathrm{C}$ NMR of $2 \mathbf{t}\left(100 \mathrm{MHz}, \mathrm{CDCl}_{3}\right)$
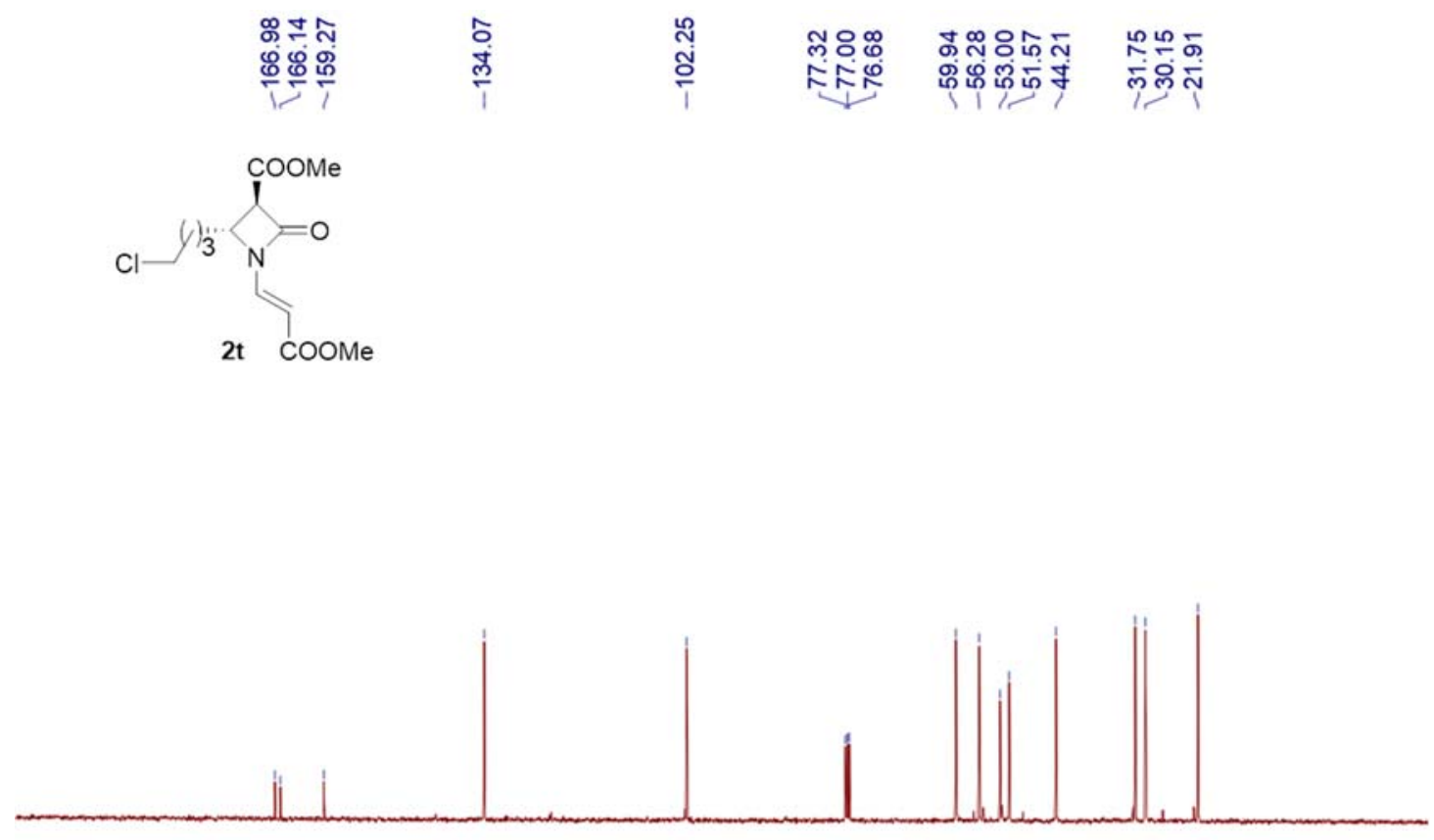

$\begin{array}{lllllllllllllll}190 & 170 & 150 & 130 & 110_{\mathrm{f} 1(\mathrm{ppm})}^{90} & 80 & 70 & 60 & 50 & 40 & 30 & 20 & 10 & 0\end{array}$ 
${ }^{1} \mathrm{H}$ NMR of $2 \mathbf{u}\left(400 \mathrm{MHz}, \mathrm{CDCl}_{3}\right)$

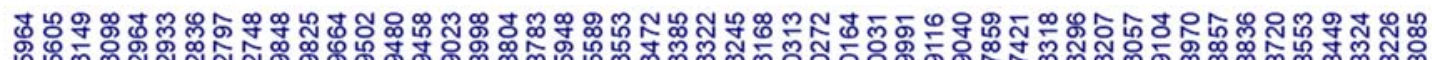

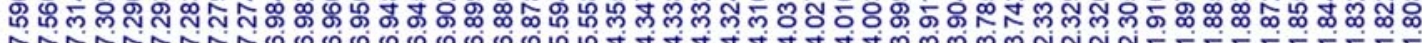

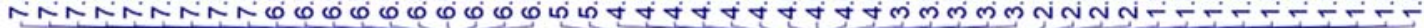<smiles>COC(=O)C=CN(CCc1ccccc1)C(=O)C(C)=O</smiles>

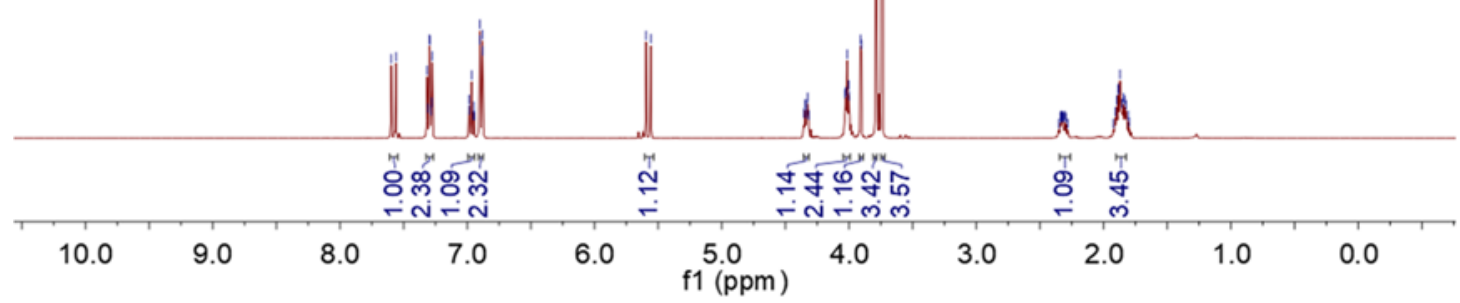

${ }^{13} \mathrm{C}$ NMR of $2 \mathbf{u}\left(100 \mathrm{MHz}, \mathrm{CDCl}_{3}\right)$

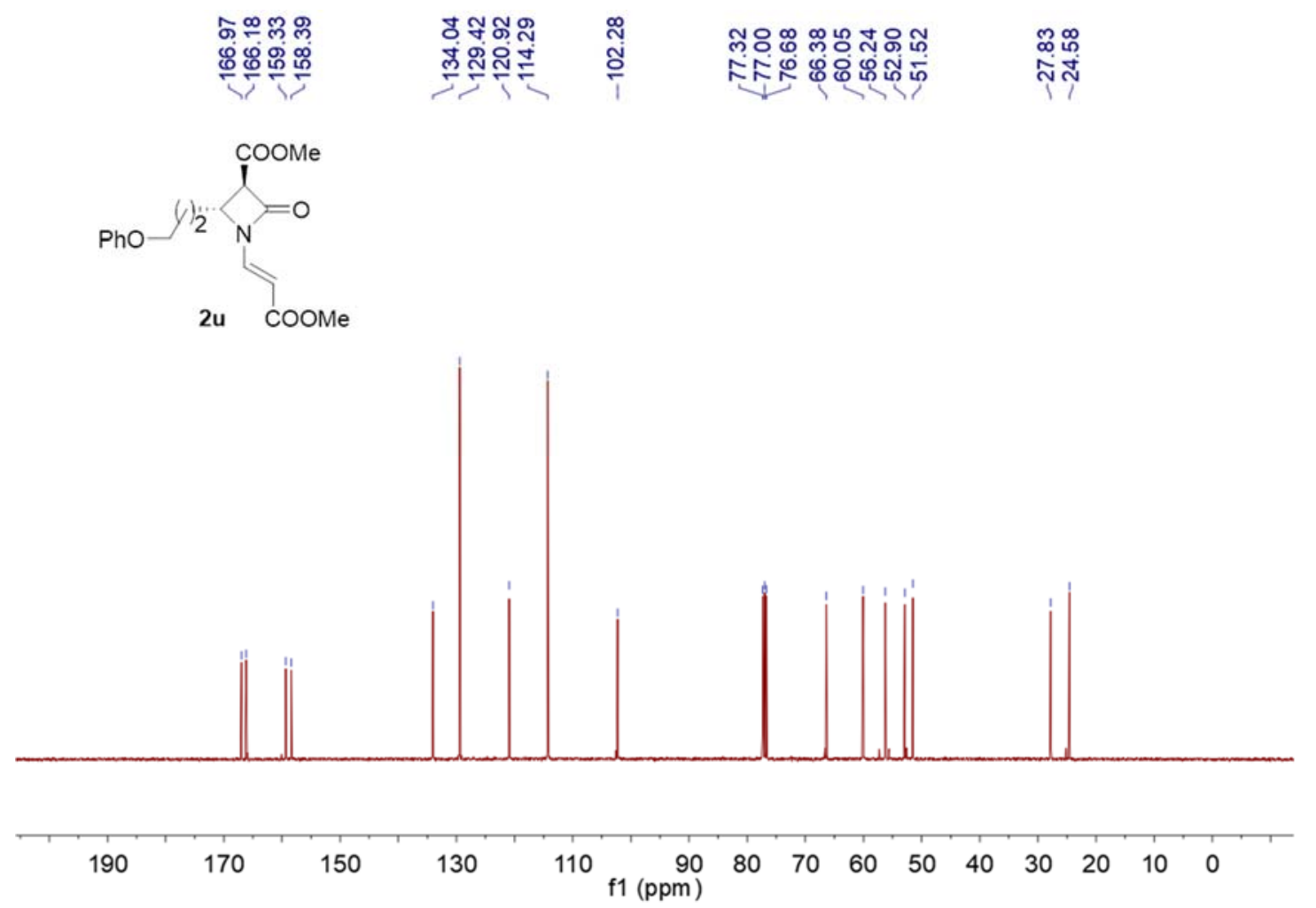


${ }^{1} \mathrm{H}$ NMR of $2 \mathbf{v}\left(400 \mathrm{MHz}, \mathrm{CDCl}_{3}\right)$
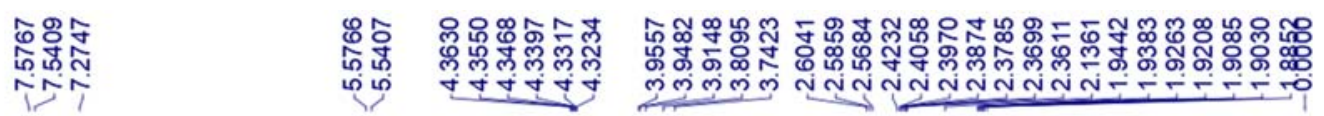

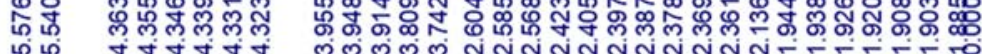

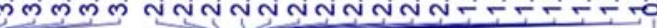
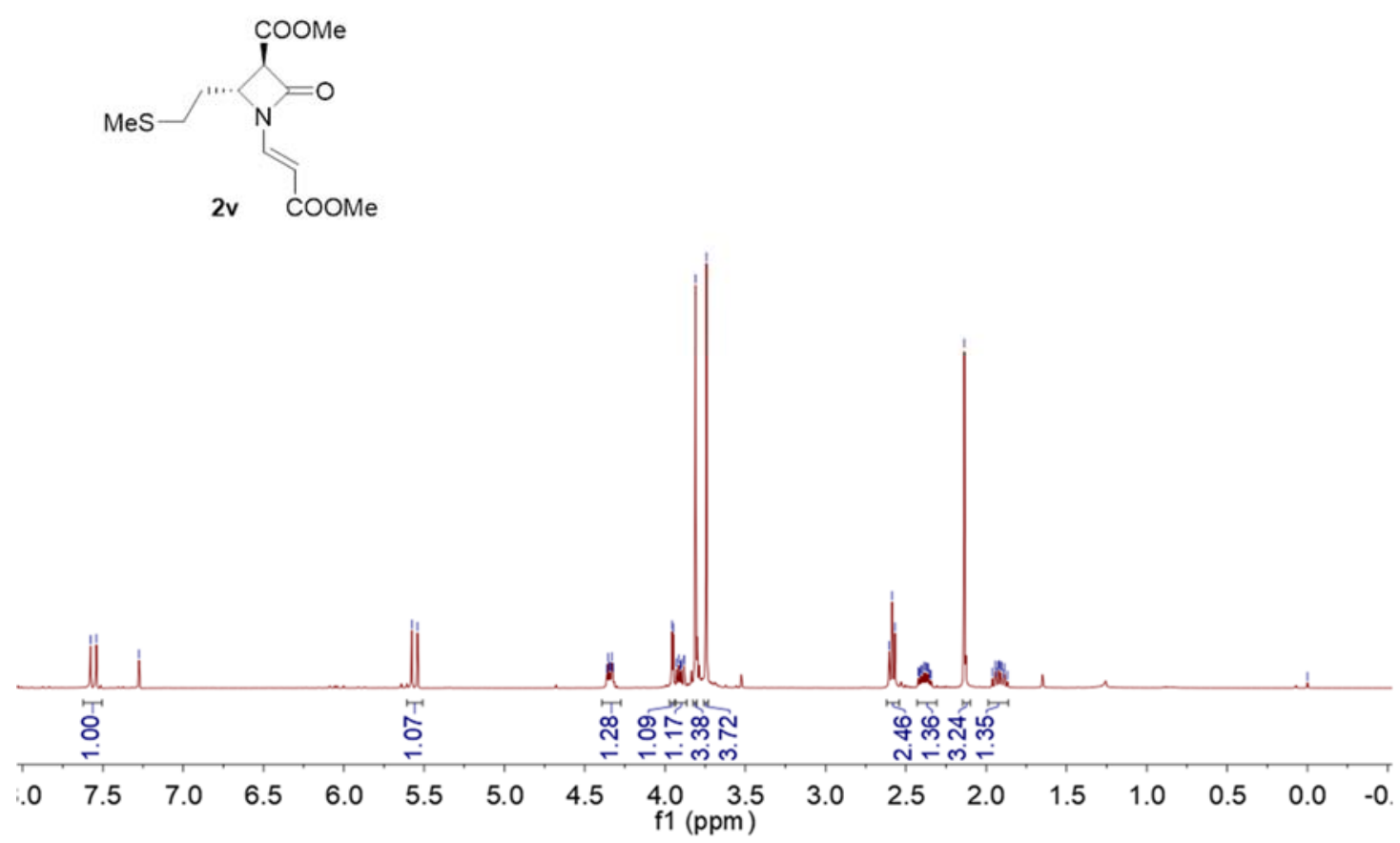

${ }^{13} \mathrm{C}$ NMR of $2 \mathbf{v}\left(100 \mathrm{MHz}, \mathrm{CDCl}_{3}\right)$

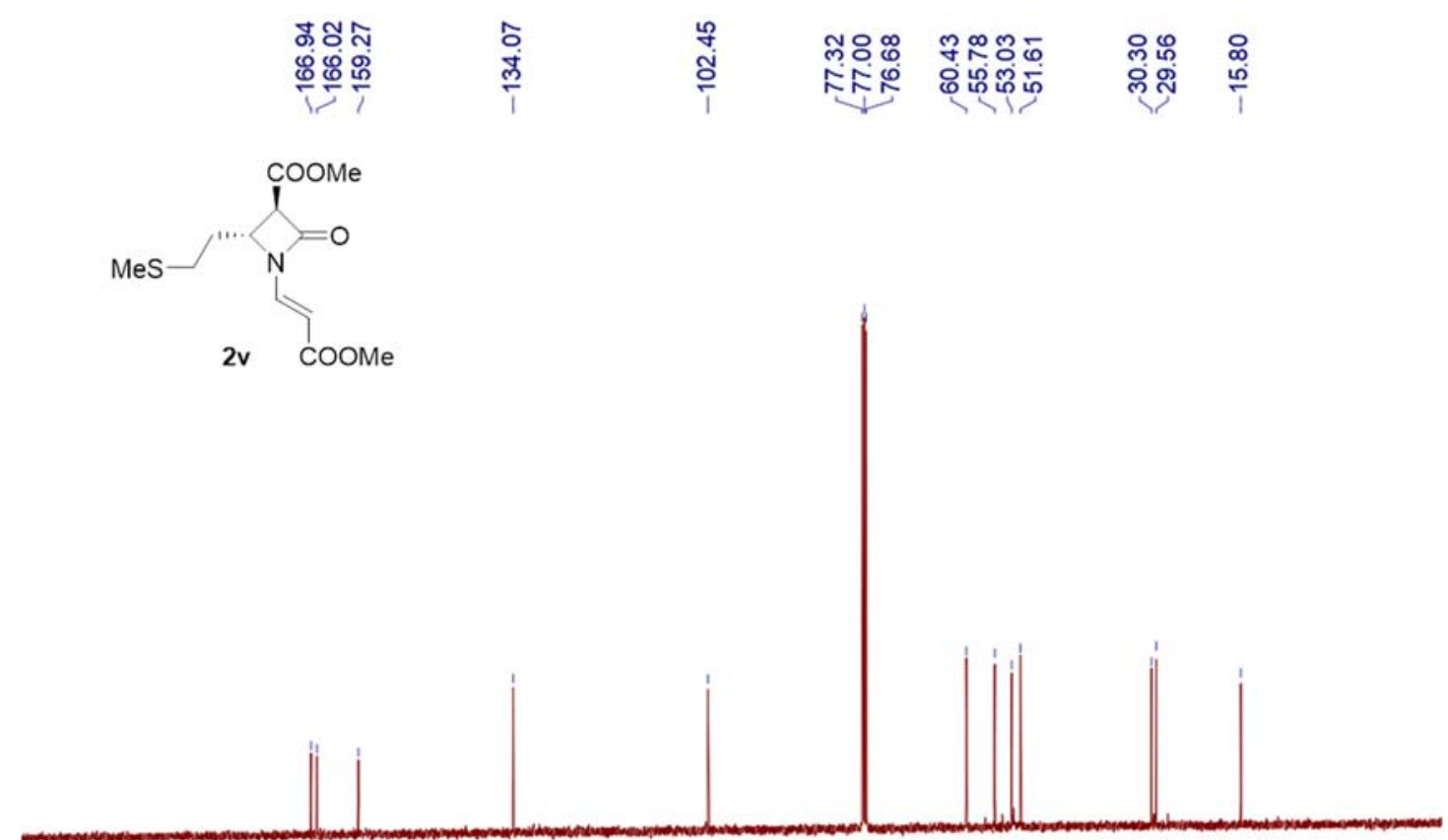

200

180

160

140

120

100
f1 $(\mathrm{ppm})$

80

60

40

20

(n) 
${ }^{1} \mathrm{H}$ NMR of $\mathbf{2 w}\left(400 \mathrm{MHz}, \mathrm{CDCl}_{3}\right)$

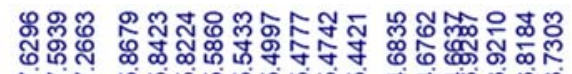

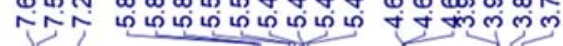
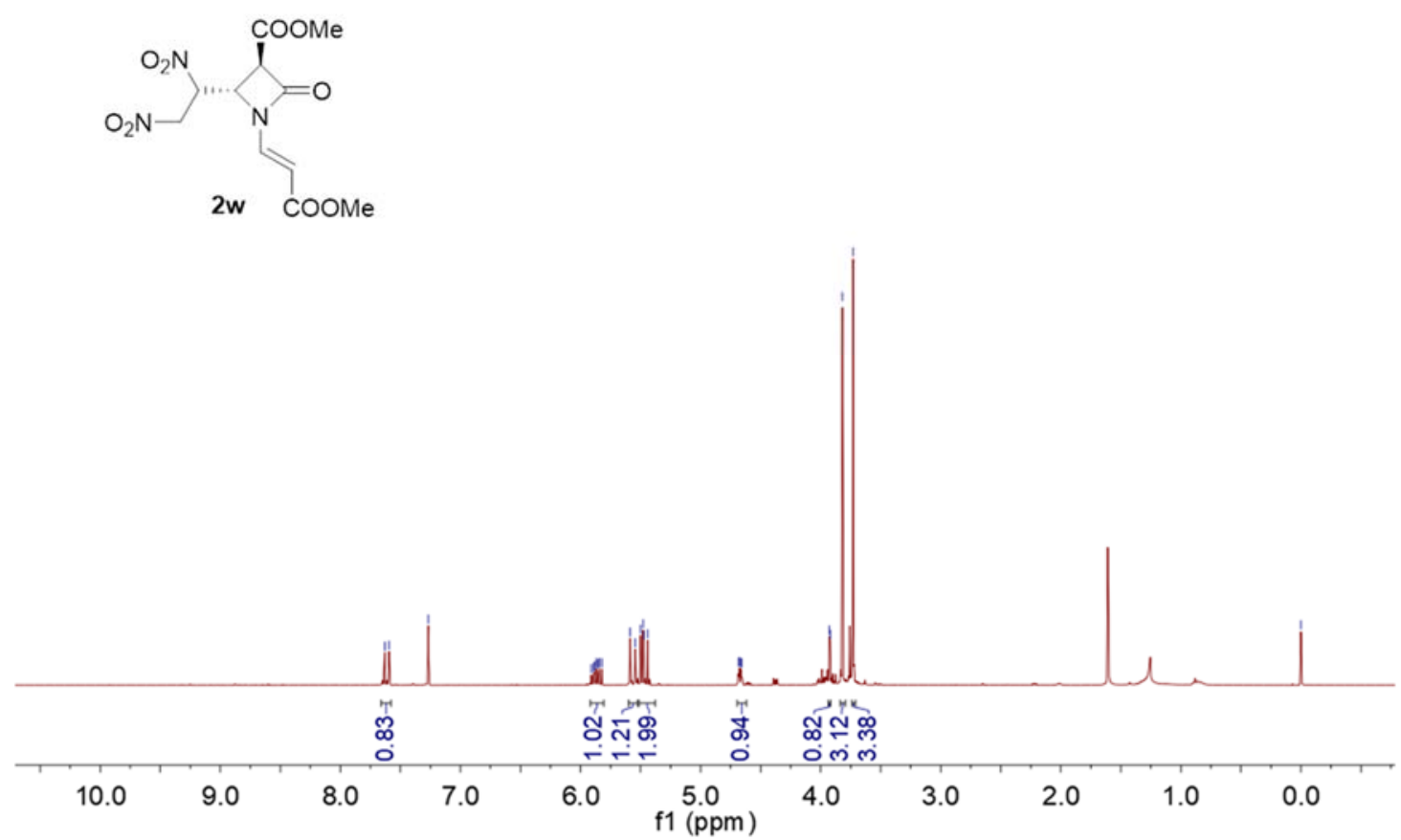

${ }^{13} \mathrm{C}$ NMR of $\mathbf{2 w}\left(100 \mathrm{MHz}, \mathrm{CDCl}_{3}\right)$

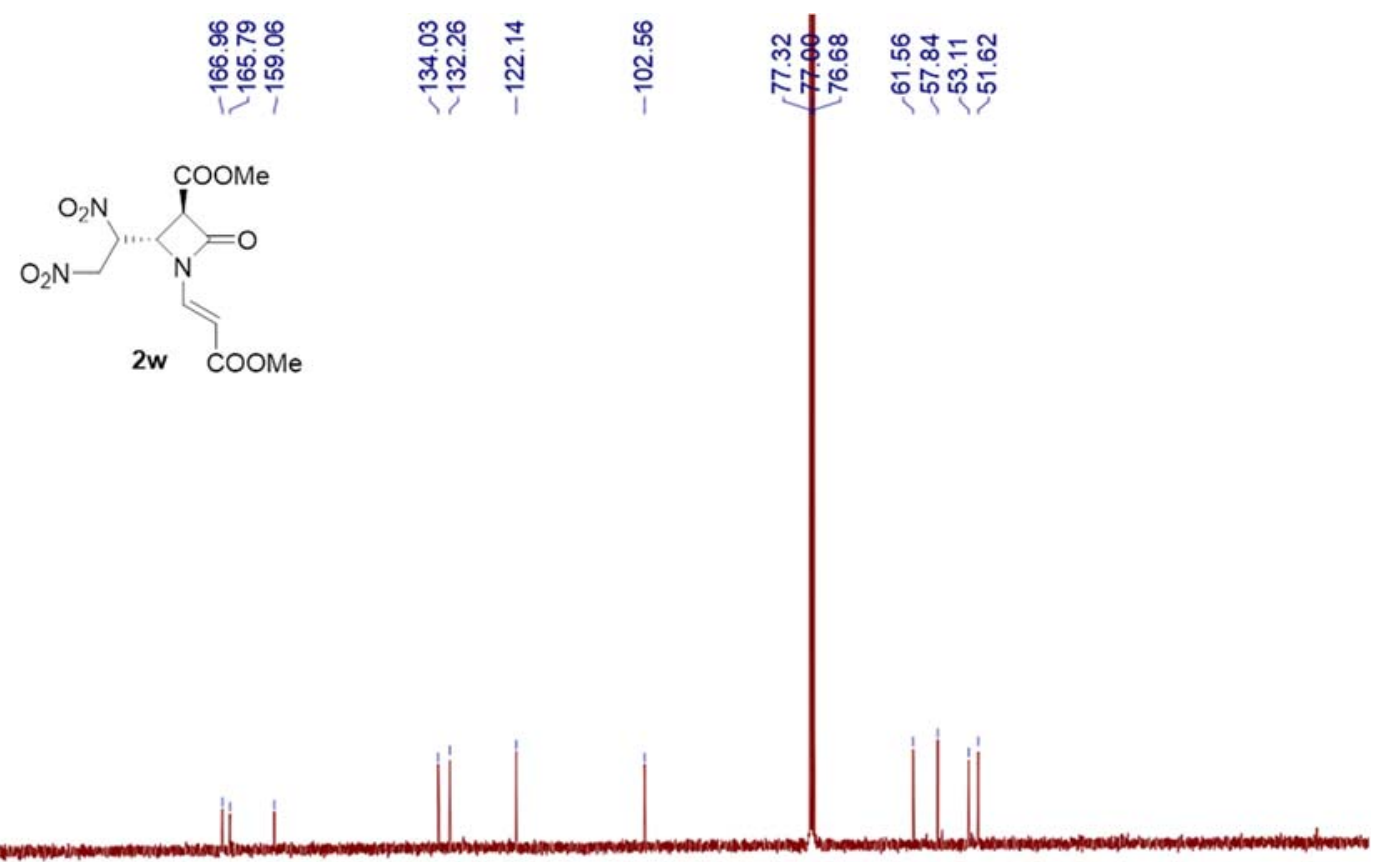

$\begin{array}{lllllllllllllllll}1 & 190 & 170 & 150 & 130 & \begin{array}{c}110 \\ \mathrm{f} 1(\mathrm{ppm})\end{array} & 90 & 80 & 70 & 60 & 50 & 40 & 30 & 20 & 10 & 0\end{array}$


${ }^{1} \mathrm{H}$ NMR of $2 \mathbf{x}\left(400 \mathrm{MHz}, \mathrm{CDCl}_{3}\right)$

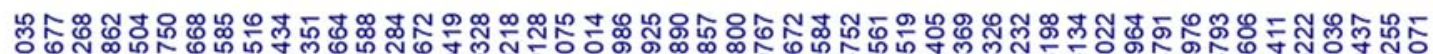

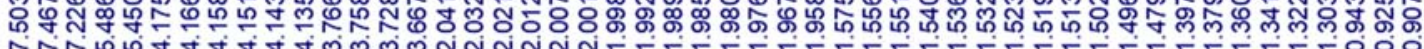

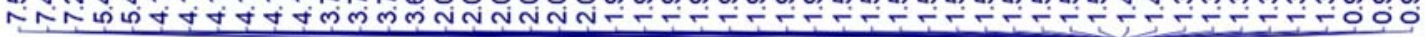<smiles>CC(=O)OC=CN1C(=O)C(C(C)C)C1C(C)C</smiles>

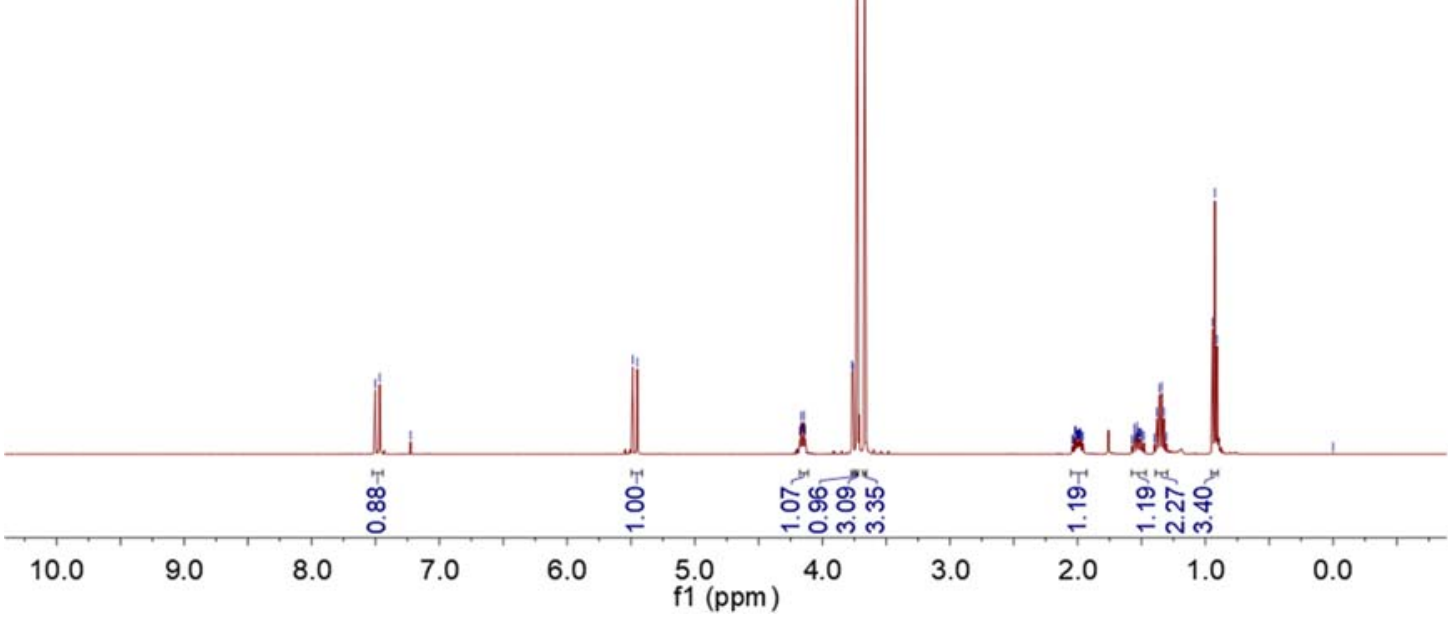

${ }^{13} \mathrm{C} \mathrm{NMR}$ of $2 \mathbf{x}\left(100 \mathrm{MHz}, \mathrm{CDCl}_{3}\right)$

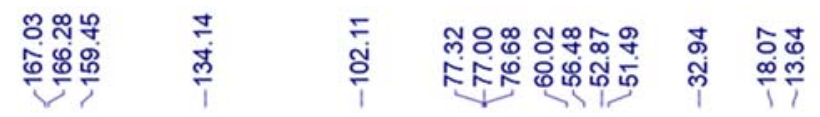<smiles>COC(=O)/C=C/N1C(=O)C(C(C)C)C1C(C)C</smiles>

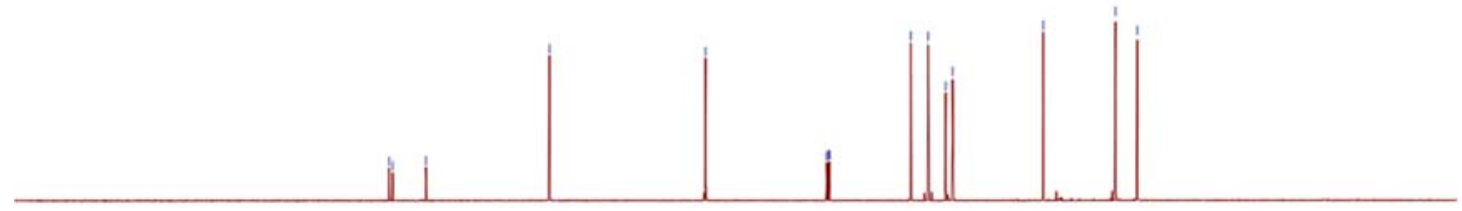

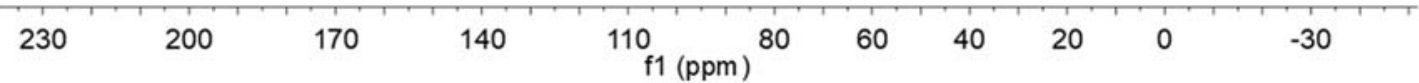


${ }^{1} \mathrm{H}$ NMR of $\mathbf{2 y}\left(400 \mathrm{MHz}, \mathrm{CDCl}_{3}\right)$

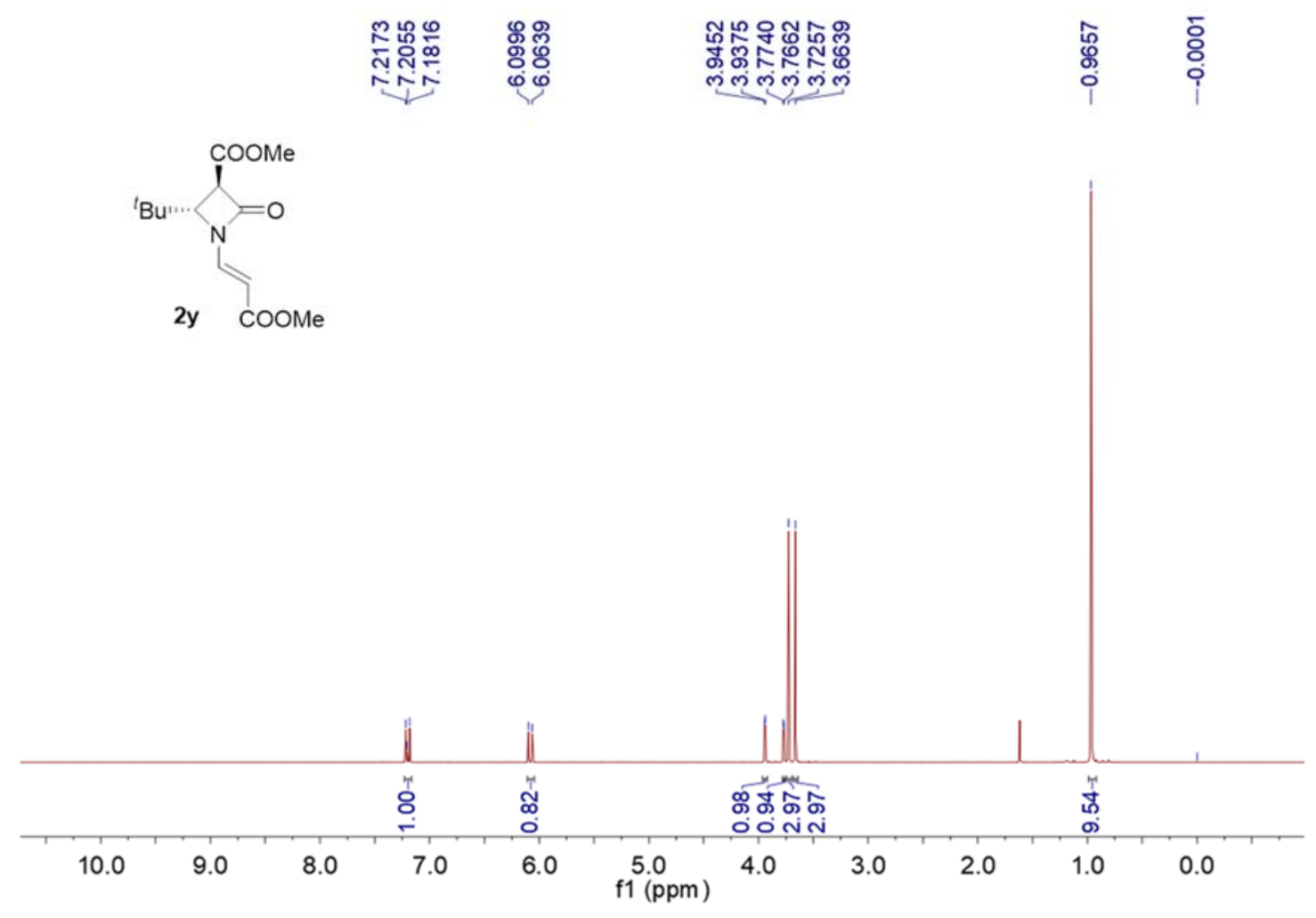

${ }^{13} \mathrm{C}$ NMR of $\mathbf{2 y}\left(100 \mathrm{MHz}, \mathrm{CDCl}_{3}\right)$

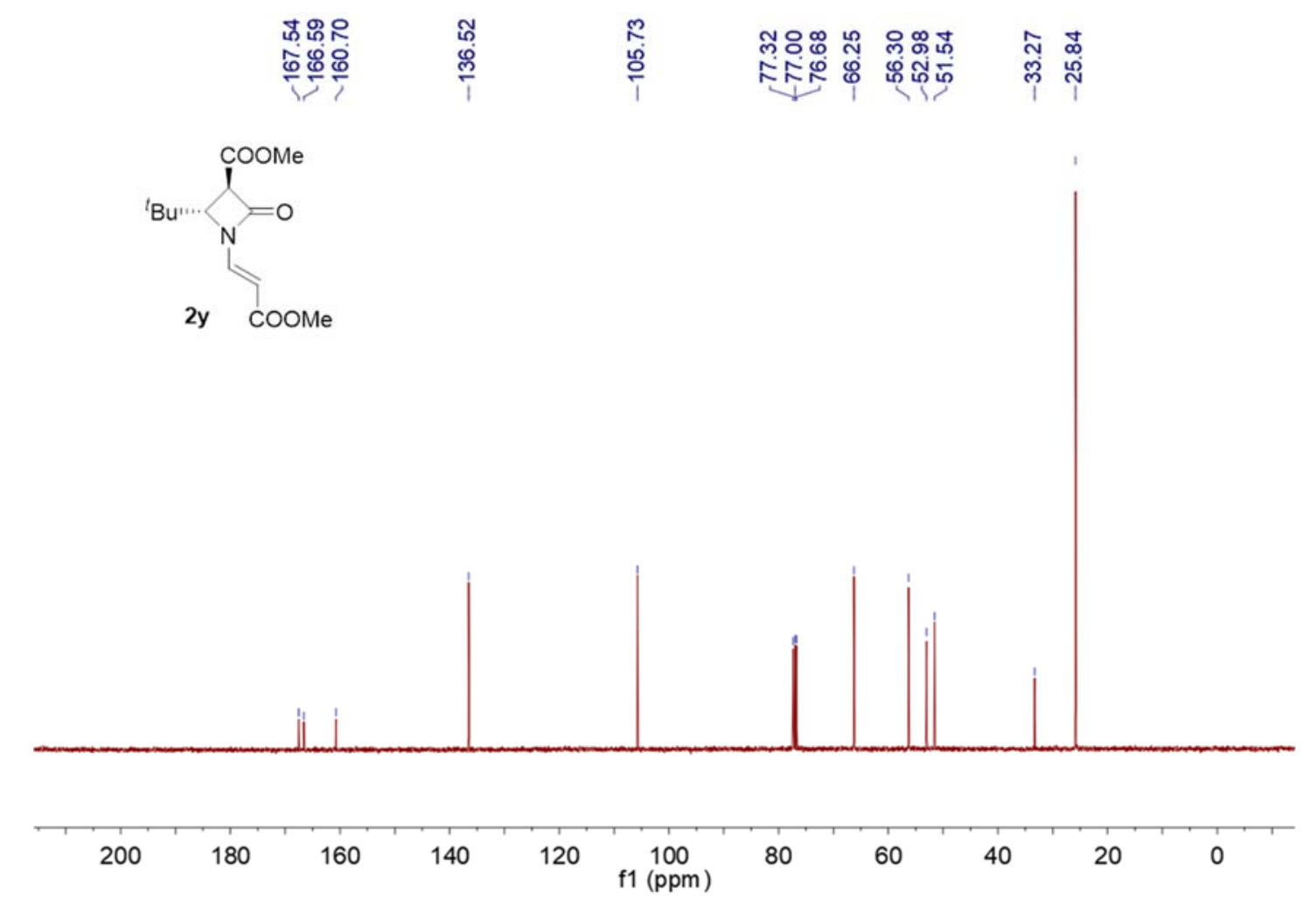


${ }^{1} \mathrm{H}$ NMR of $\mathbf{2 z}\left(400 \mathrm{MHz}, \mathrm{CDCl}_{3}\right)$
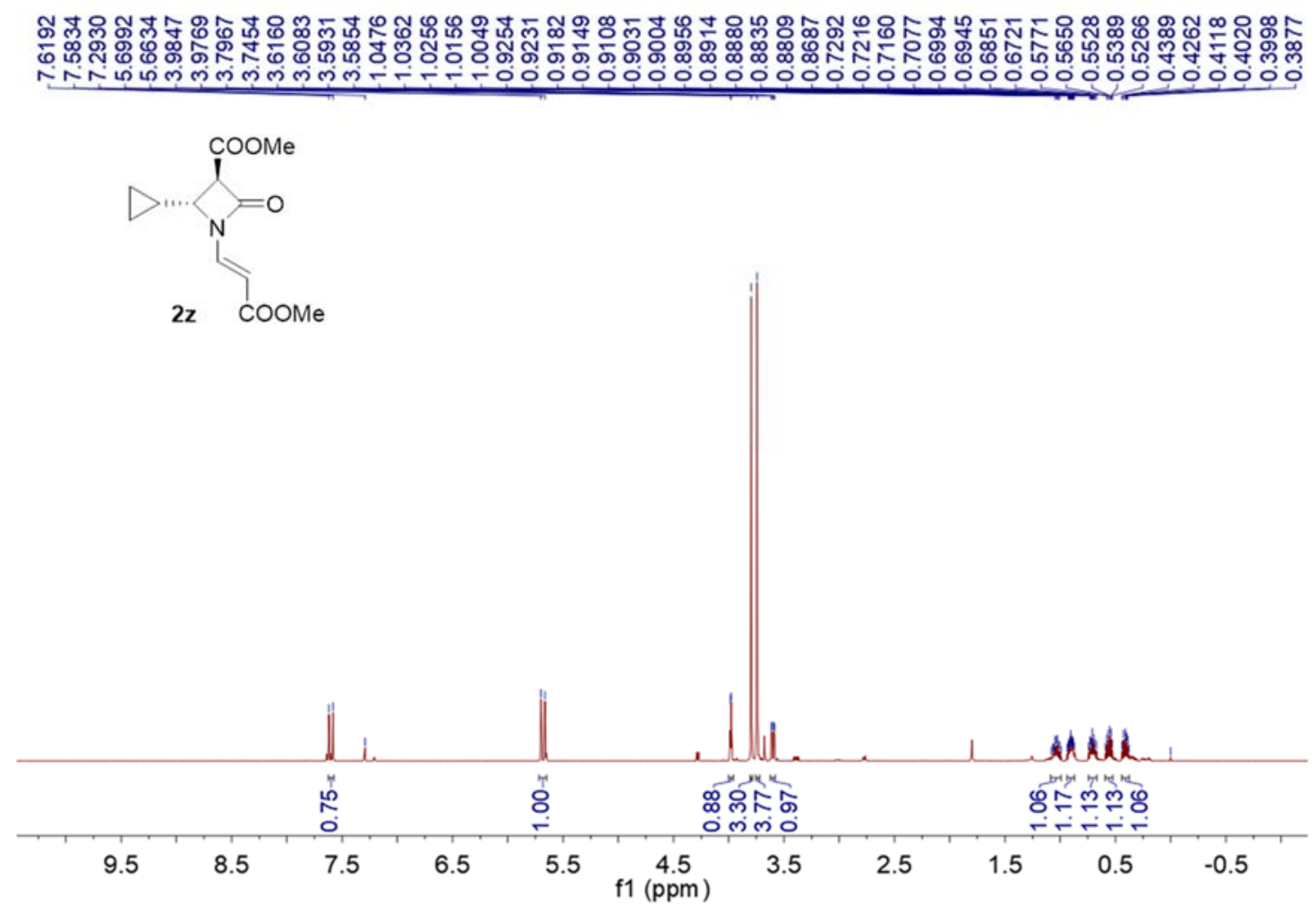

${ }^{13} \mathrm{C}$ NMR of $\mathbf{2 z}\left(100 \mathrm{MHz}, \mathrm{CDCl}_{3}\right)$

\begin{tabular}{|c|c|c|c|}
\hline 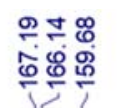 & $\begin{array}{l}\text { ָั } \\
\text { J }\end{array}$ & 훙 & 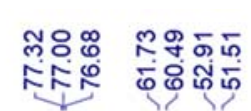 \\
\hline
\end{tabular}
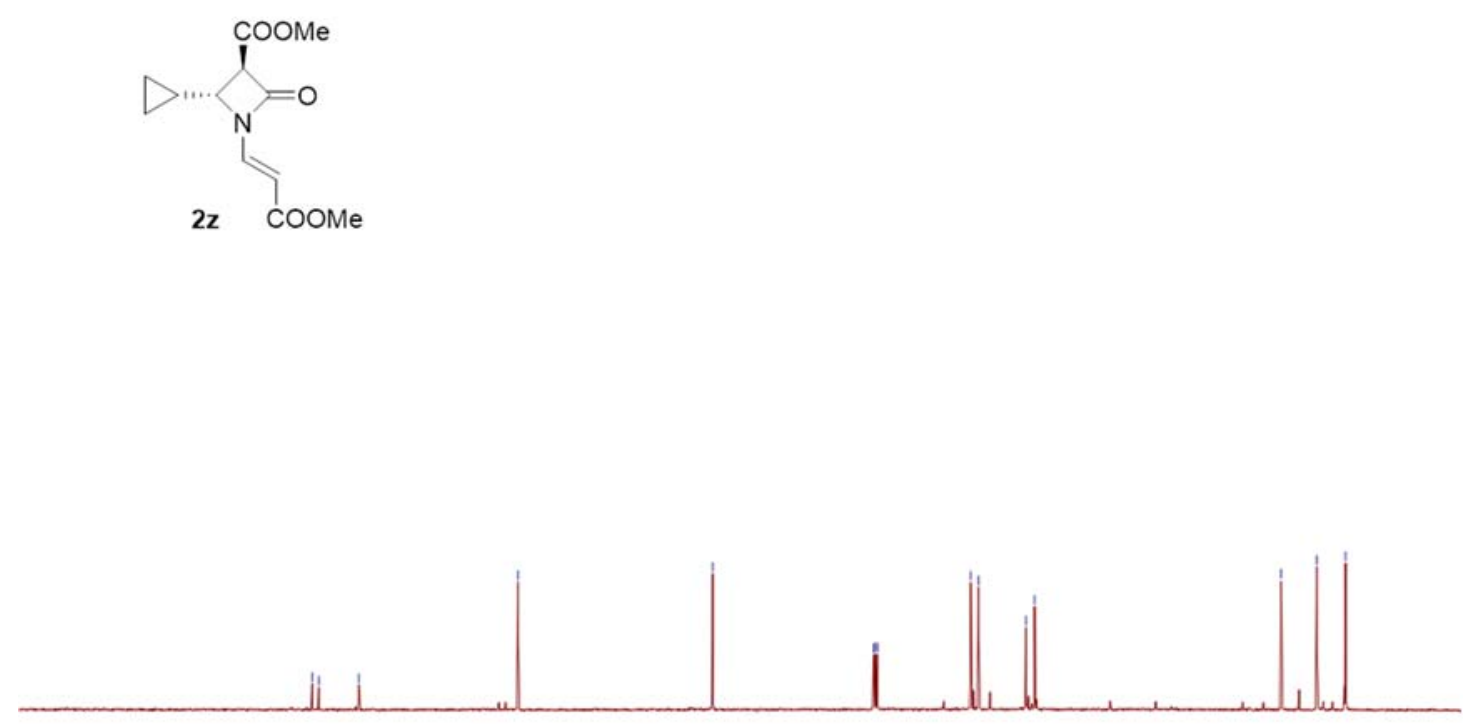

$\left.200 \quad 180 \quad 160 \quad 140 \quad 120 \begin{array}{c}100 \\ \mathrm{f} 1(\mathrm{ppm})\end{array}\right)$ 
${ }^{1} \mathrm{H}$ NMR of 2aa $\left(400 \mathrm{MHz}, \mathrm{CDCl}_{3}\right)$
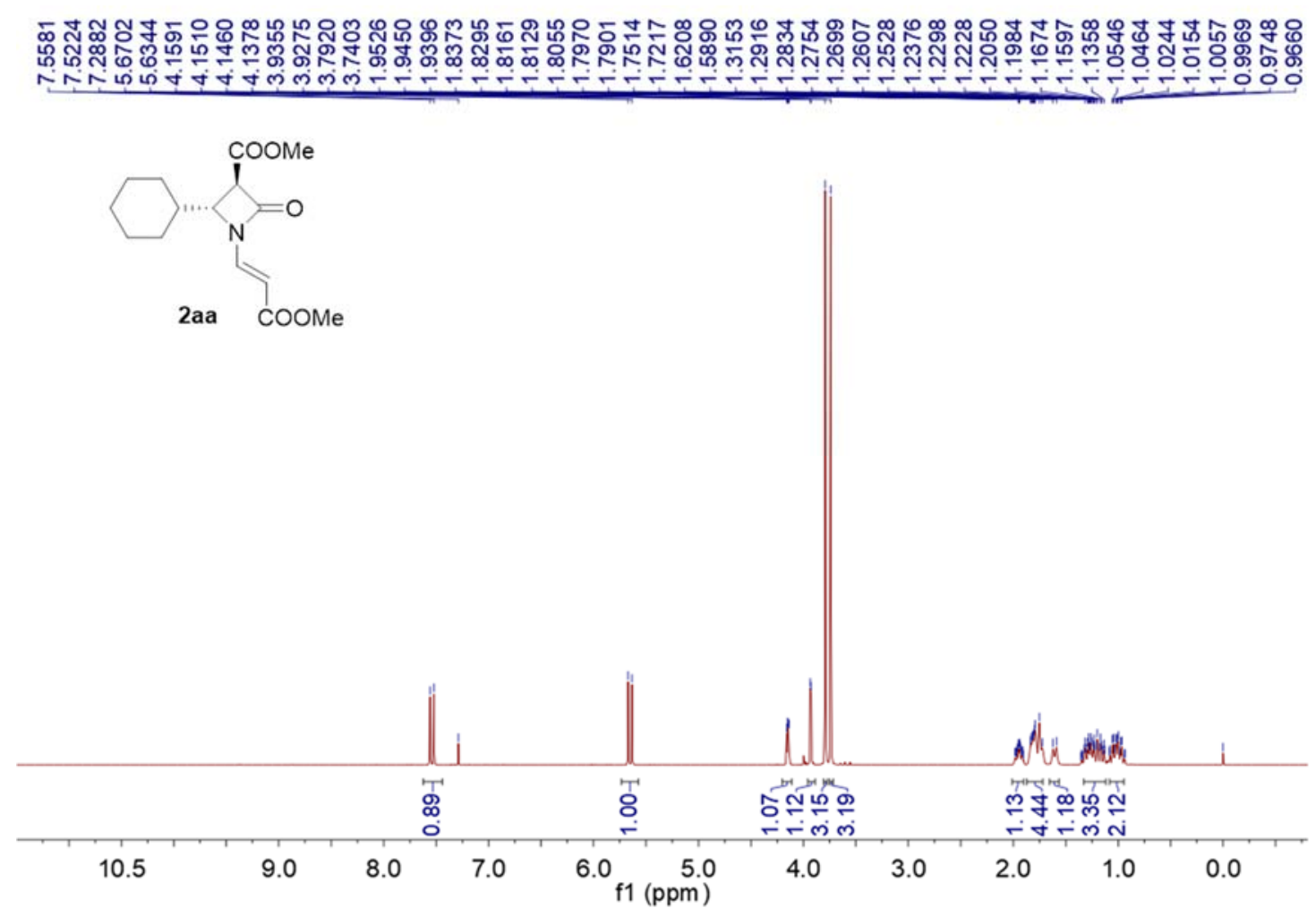

${ }^{13} \mathrm{C}$ NMR of $2 \mathbf{a a}\left(100 \mathrm{MHz}, \mathrm{CDCl}_{3}\right)$

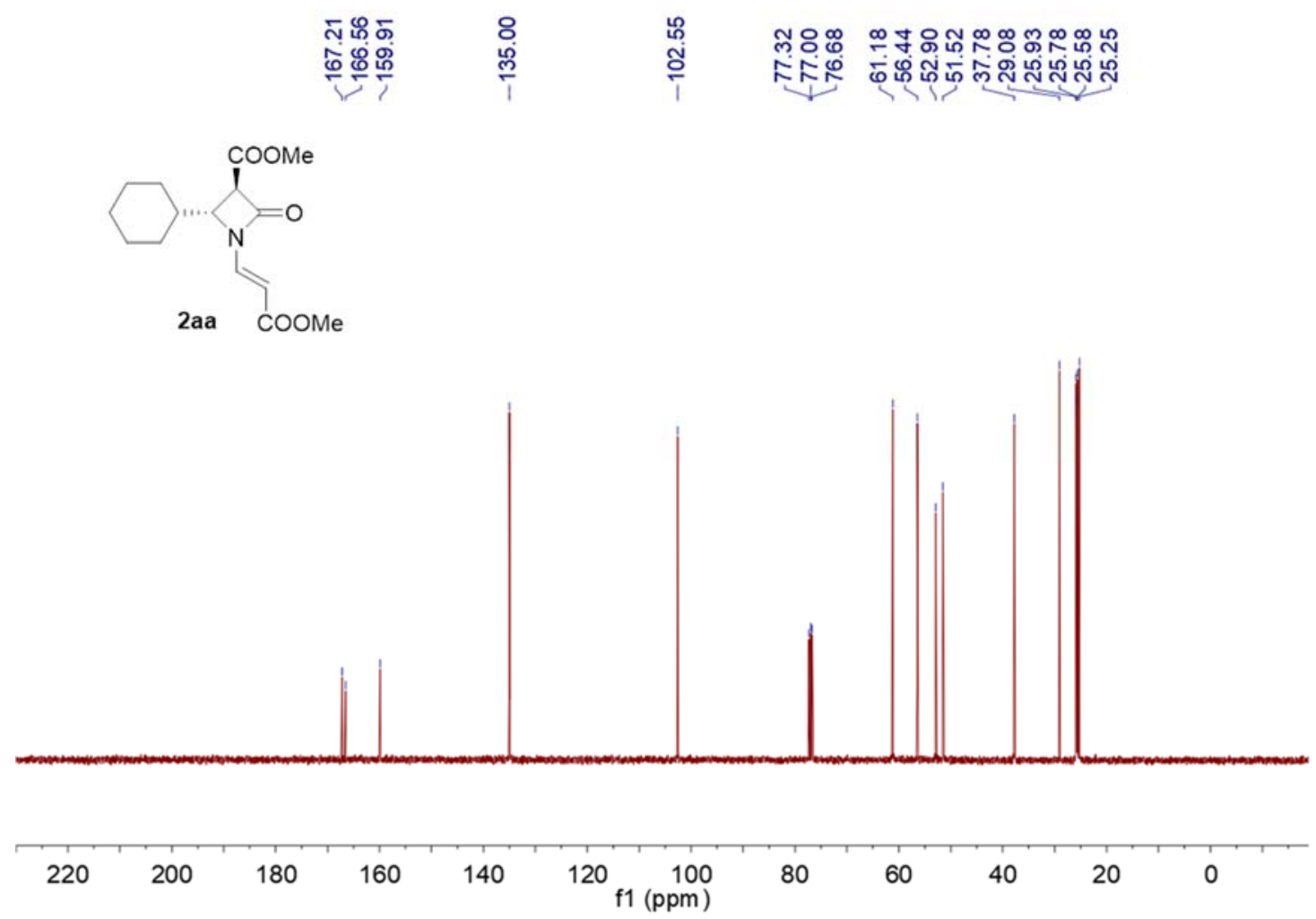


${ }^{1} \mathrm{H}$ NMR of $\mathbf{2 a b}\left(400 \mathrm{MHz}, \mathrm{CDCl}_{3}\right)$

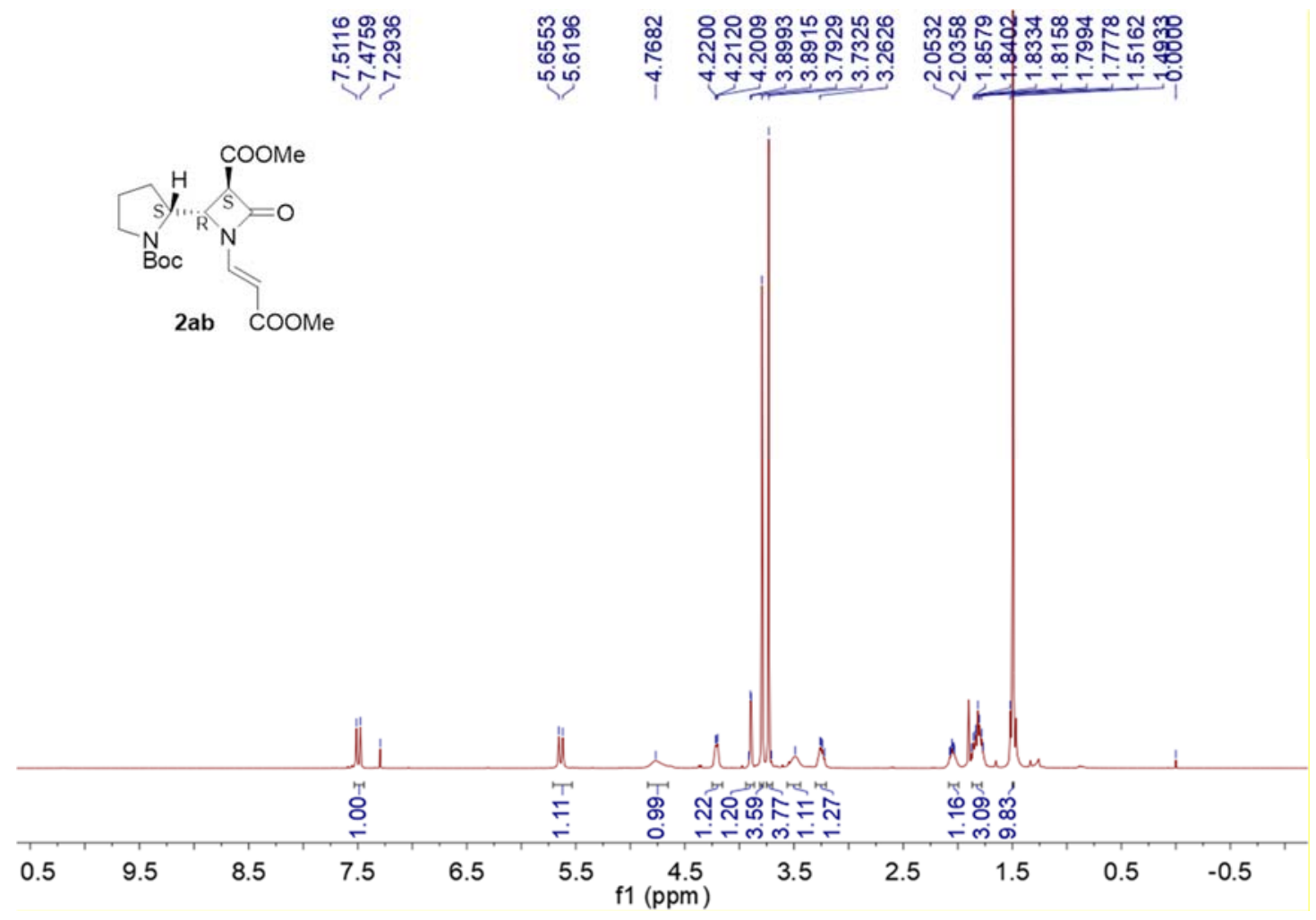

${ }^{13} \mathrm{C}$ NMR of $2 \mathbf{a b}\left(100 \mathrm{MHz}, \mathrm{CDCl}_{3}\right)$

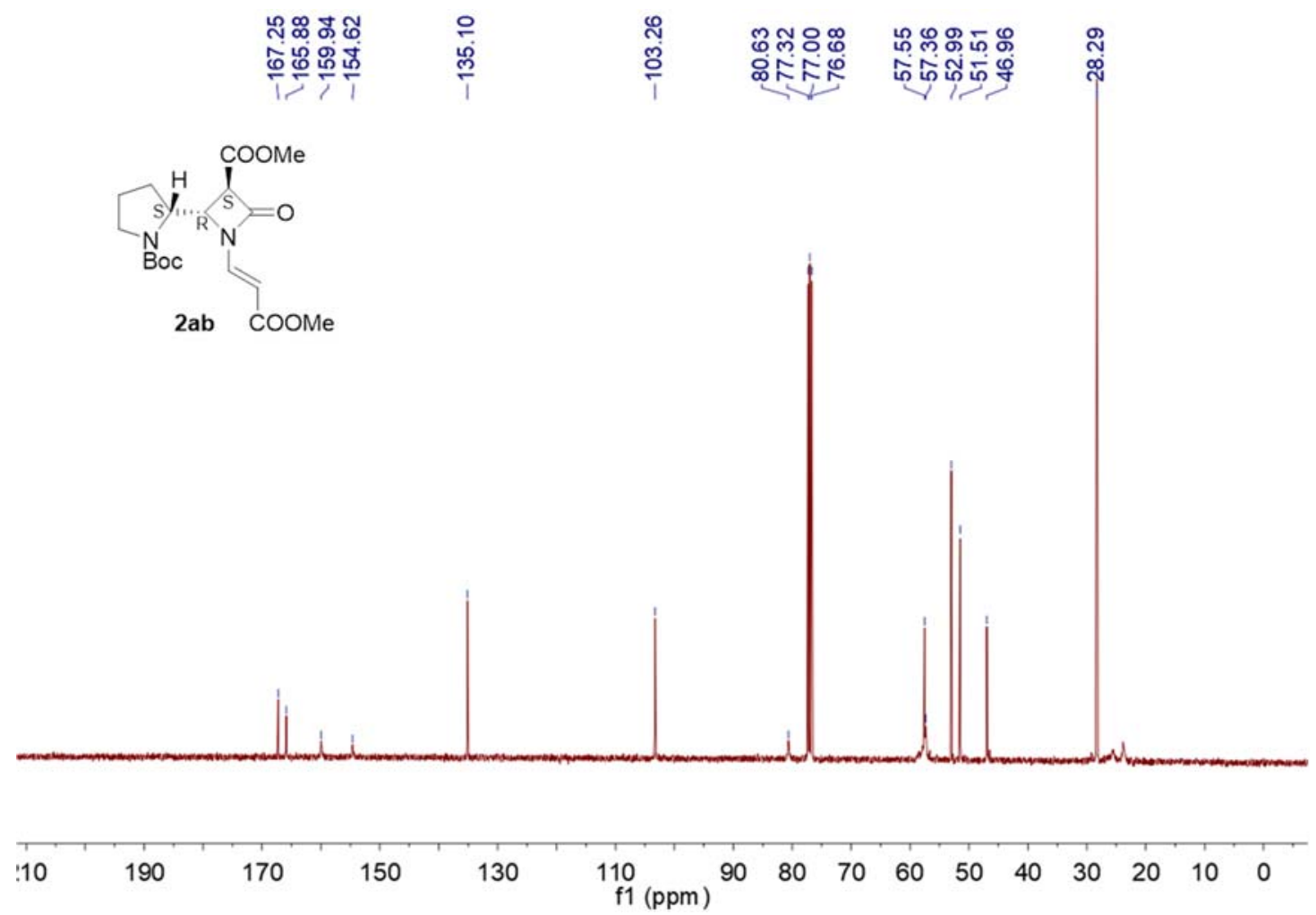




\begin{tabular}{|r|r|l|r|r|r|}
\hline \multicolumn{1}{|c|}{ Peak\# } & Ret. Time & Area & Height & \multicolumn{1}{|c|}{ Area \% } & \multicolumn{1}{c|}{ Height \% } \\
\hline 1 & 29.941 & 40936298 & 193392 & 52.567 & 58.963 \\
\hline 2 & 49.695 & 36938821 & 134595 & 47.433 & 41.037 \\
\hline Total & & 77875119 & 327986 & 100.000 & 100.000 \\
\hline
\end{tabular}

$\mathrm{mV}$

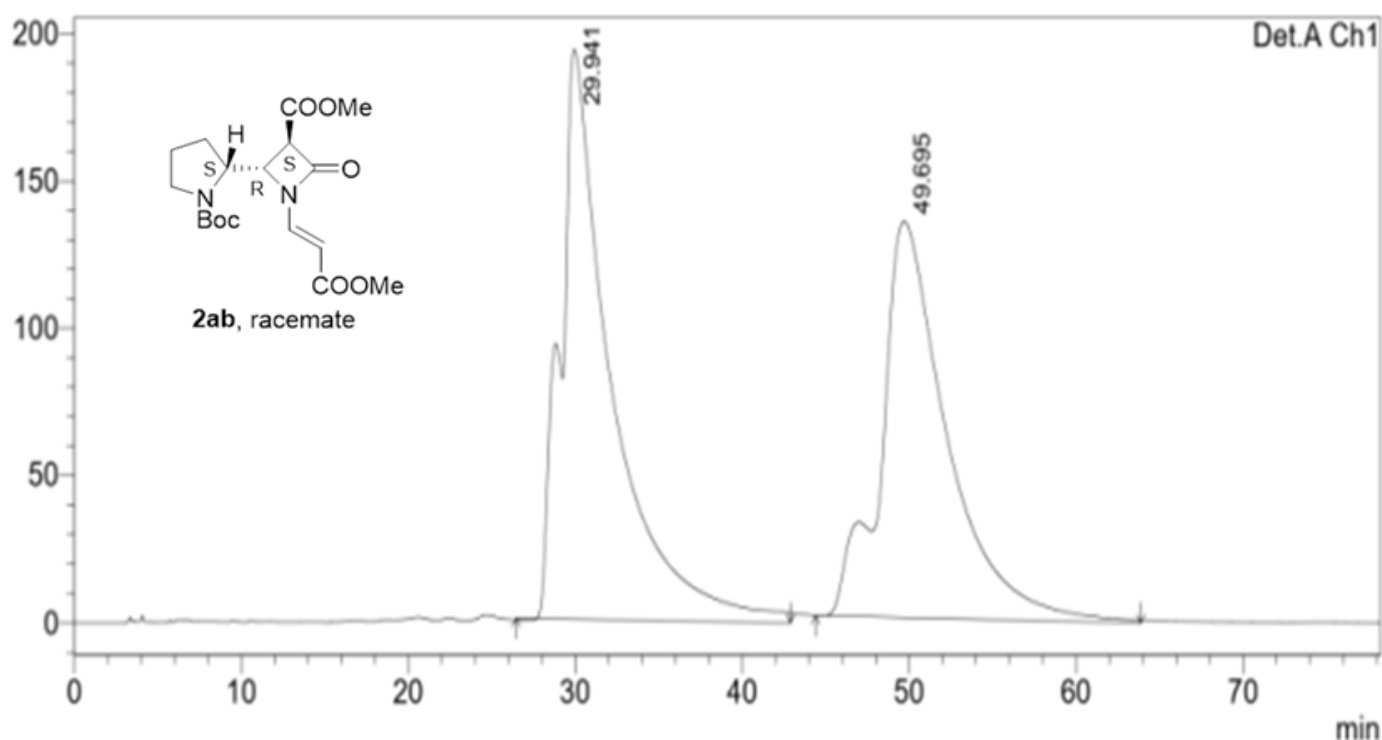

PeakTable

Detector A Chl 254nm

\begin{tabular}{|r|r|r|r|r|r|}
\hline \multicolumn{1}{|c|}{ Peak\# } & Ret. Time & \multicolumn{1}{|c|}{ Area } & Height & Area \% & \multicolumn{1}{c|}{ Height \% } \\
\hline 1 & 33.427 & 1925904 & 9751 & 2.589 & 3.166 \\
\hline 2 & 48.340 & 72458960 & 298220 & 97.411 & 96.834 \\
\hline Total & & 74384865 & 307971 & 100.000 & 100.000 \\
\hline
\end{tabular}

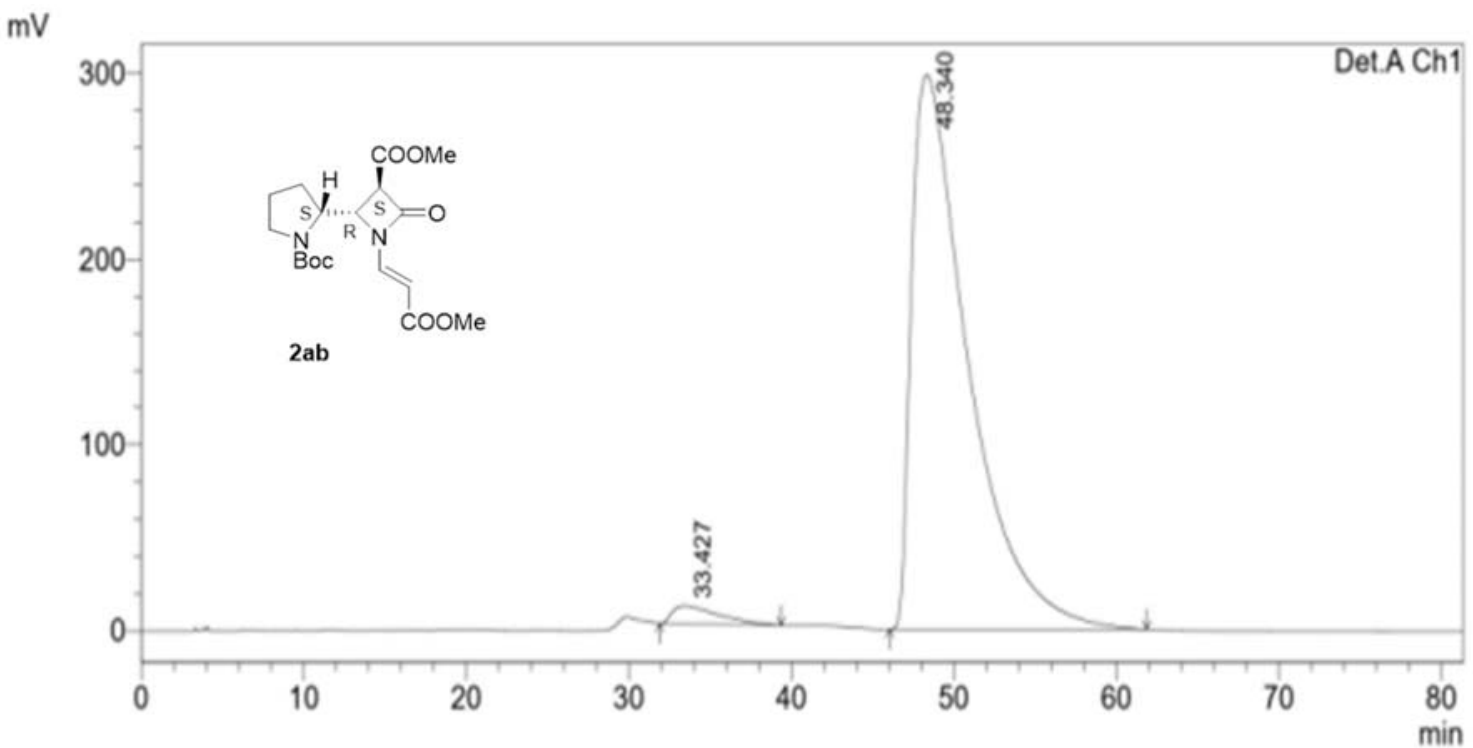


${ }^{1} \mathrm{H}$ NMR of $\mathbf{2 a c}\left(400 \mathrm{MHz}, \mathrm{CDCl}_{3}\right)$

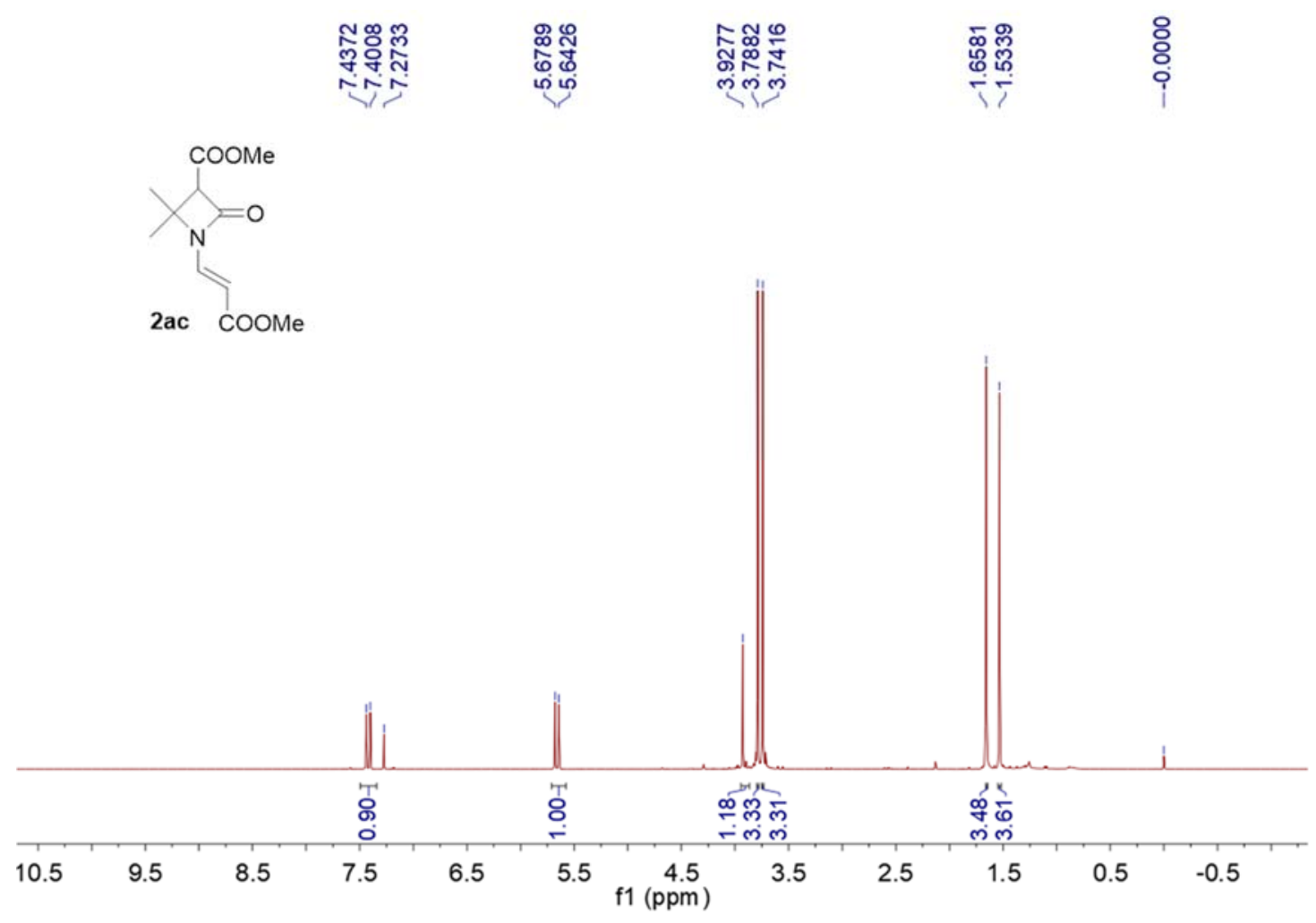

${ }^{13} \mathrm{C}$ NMR of $2 \mathbf{a c}\left(100 \mathrm{MHz}, \mathrm{CDCl}_{3}\right)$

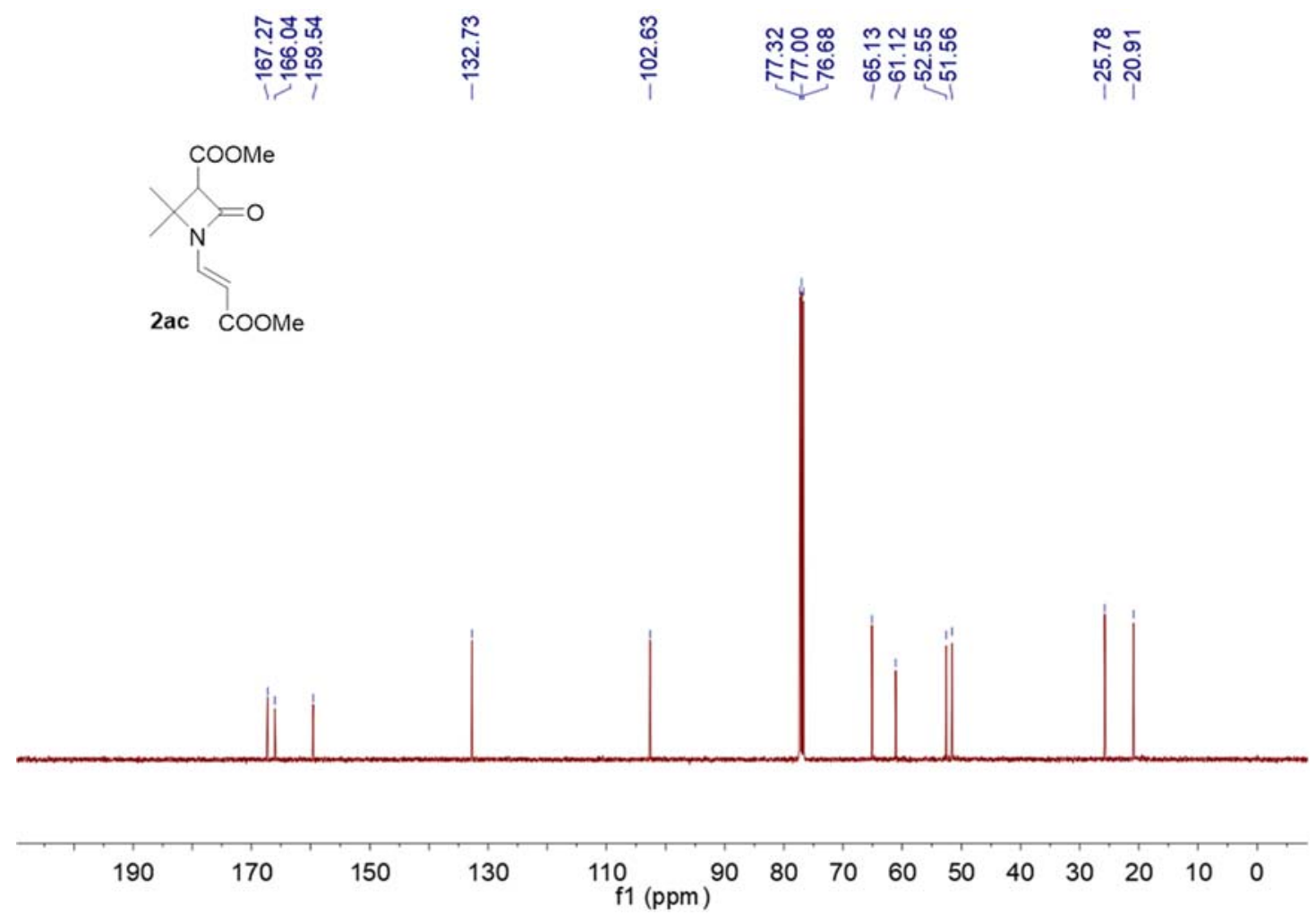


${ }^{1} \mathrm{H}$ NMR of $\mathbf{2 a d}\left(400 \mathrm{MHz}, \mathrm{CDCl}_{3}\right)$

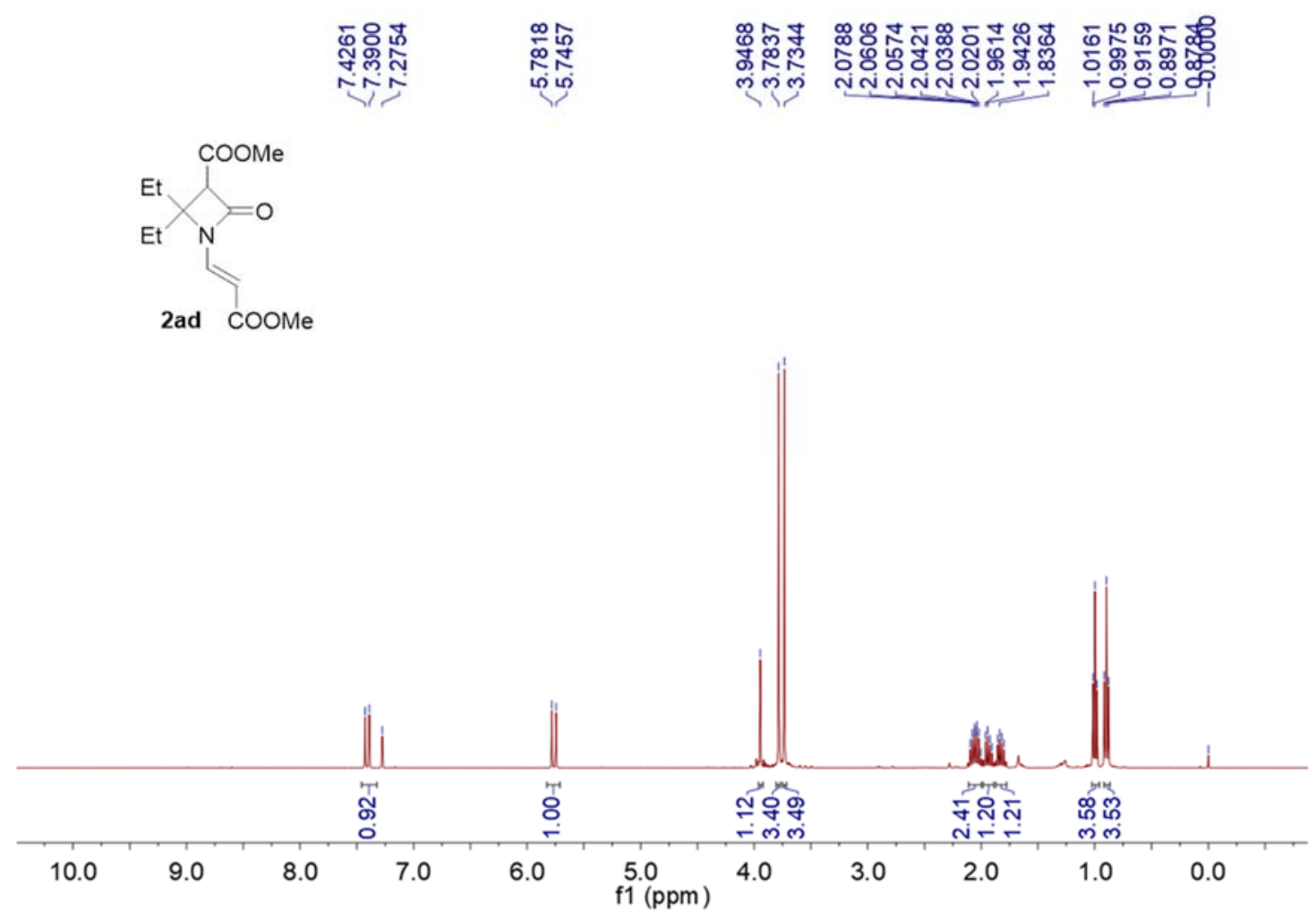

${ }^{13} \mathrm{C}$ NMR of $\mathbf{2 a d}\left(100 \mathrm{MHz}, \mathrm{CDCl}_{3}\right)$

\begin{tabular}{|c|c|c|c|c|}
\hline 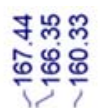 & 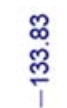 & 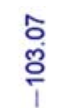 & 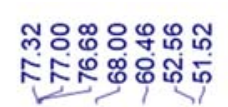 & 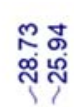 \\
\hline
\end{tabular}

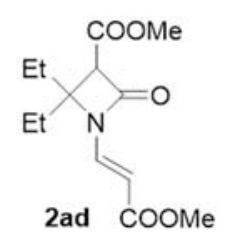

\begin{tabular}{llllllllllllllllll}
\hline 210 & 190 & 170 & 150 & 130 & $\begin{array}{c}110 \\
\mathrm{f} 1(\mathrm{ppm})\end{array}$ & 90 & 80 & 70 & 60 & 50 & 40 & 30 & 20 & 10 & 0
\end{tabular}


${ }^{1} \mathrm{H}$ NMR of $\mathbf{2 a e}\left(400 \mathrm{MHz}, \mathrm{CDCl}_{3}\right)$
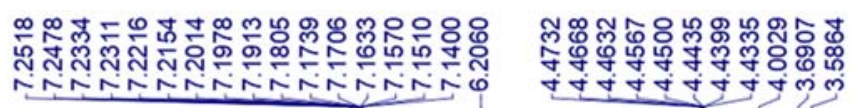

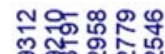
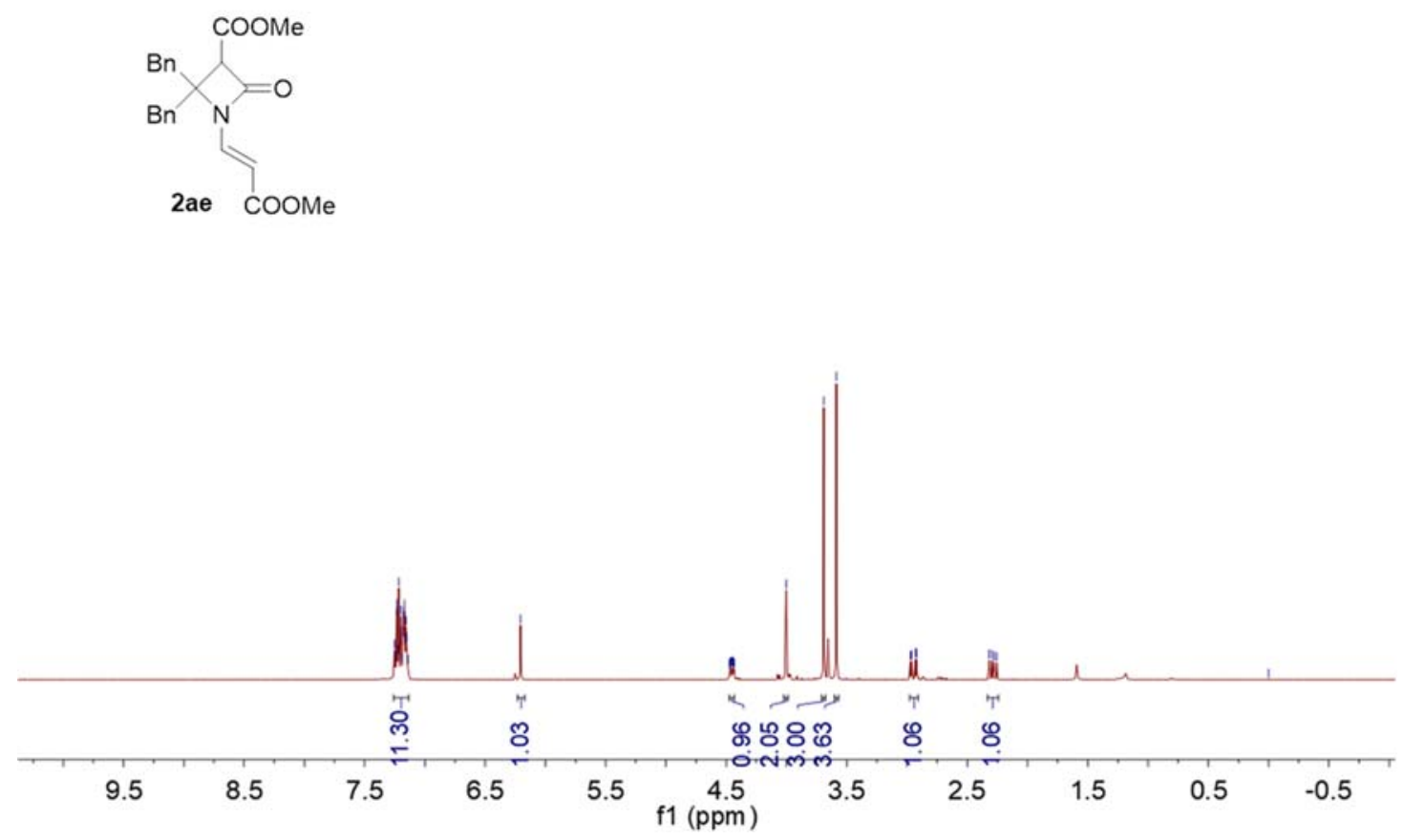

${ }^{13} \mathrm{C}$ NMR of $2 \mathbf{a e}\left(100 \mathrm{MHz}, \mathrm{CDCl}_{3}\right)$

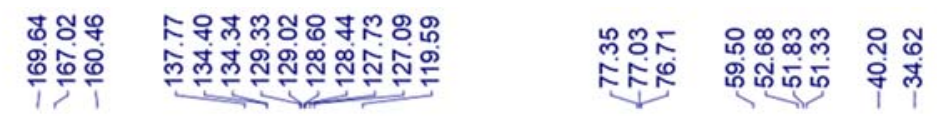
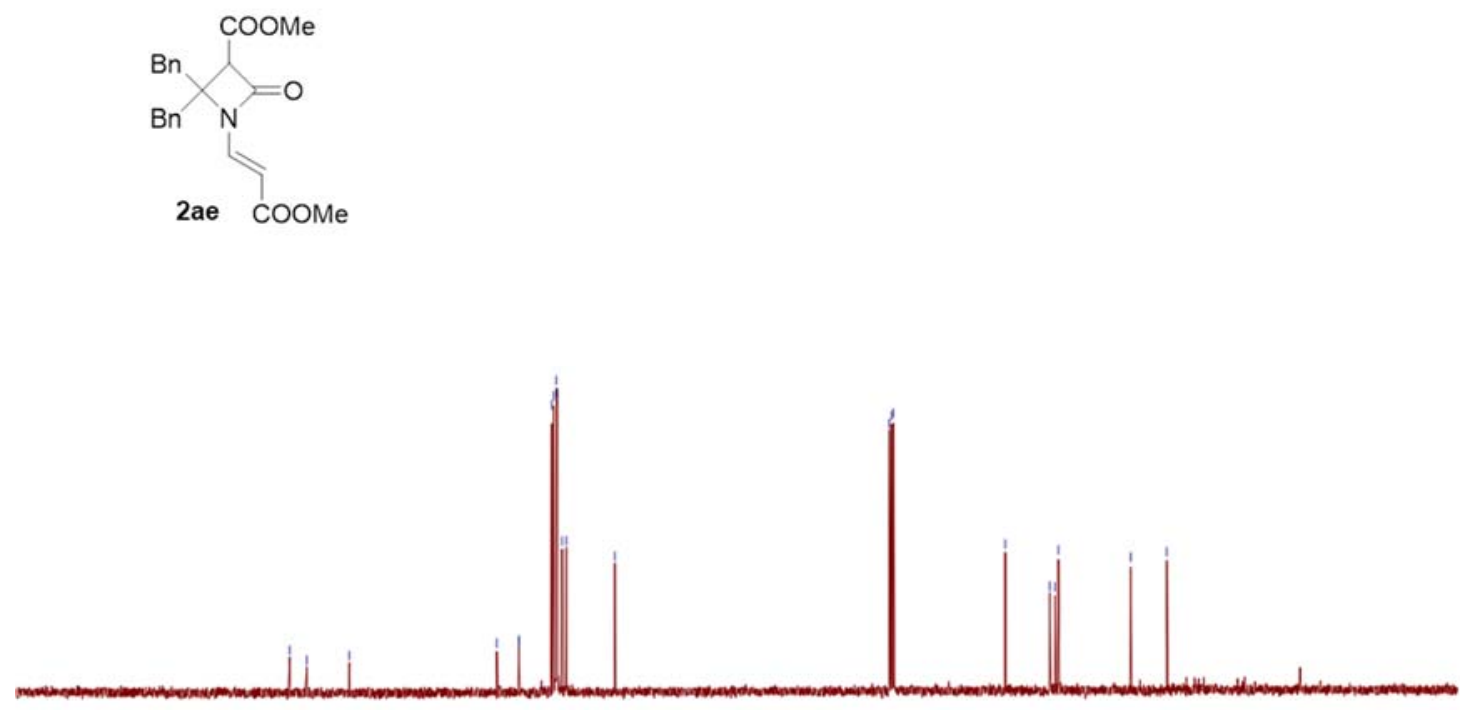

$\begin{array}{llllllllllllllll}10 & 190 & 170 & 150 & 130 & 110 & 90 & 80 & 70 & 60 & 50 & 40 & 30 & 20 & 10 & 0\end{array}$


${ }^{1} \mathrm{H}$ NMR of $\mathbf{2 a f}\left(400 \mathrm{MHz}, \mathrm{CDCl}_{3}\right)$

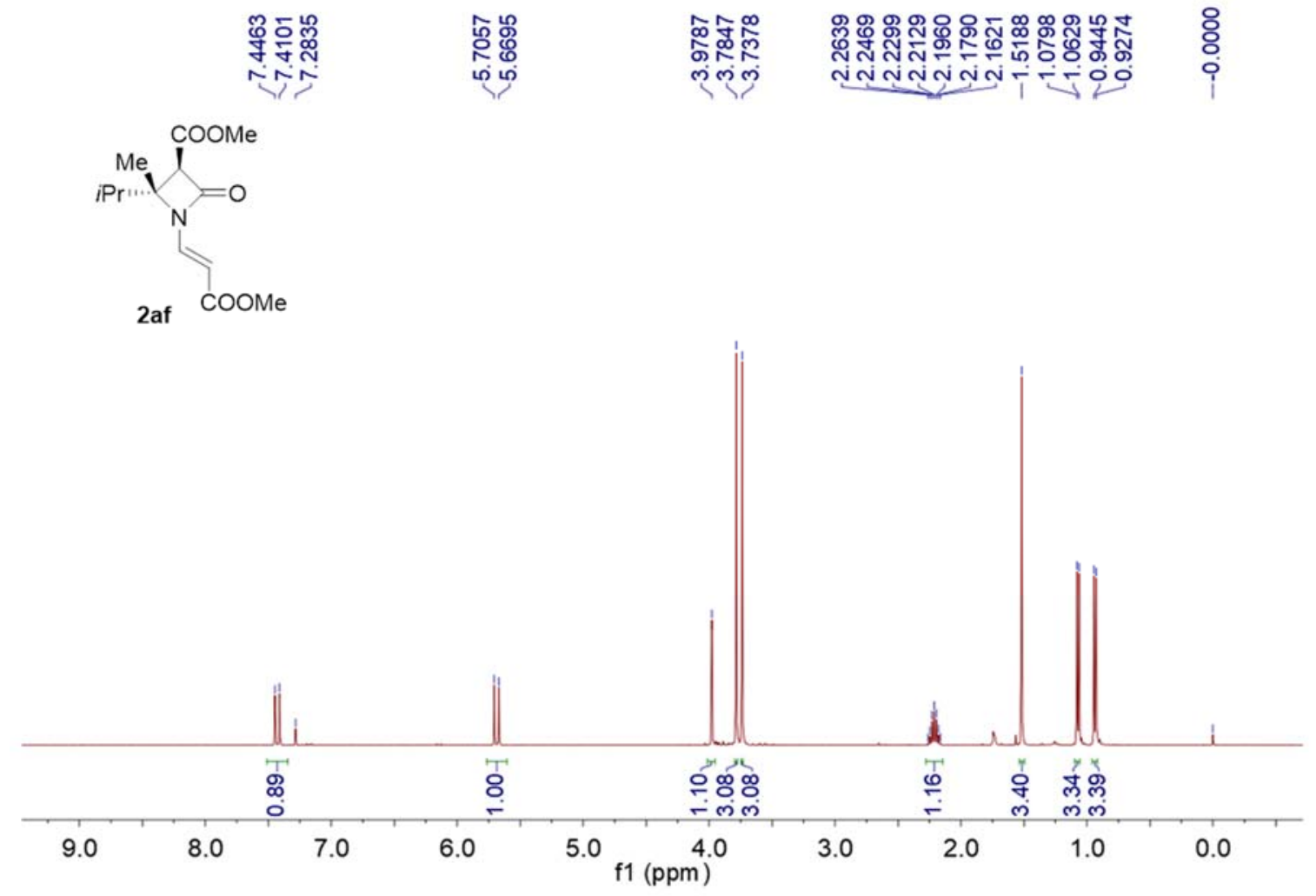

${ }^{13} \mathrm{C}$ NMR of $2 \mathbf{a f}\left(100 \mathrm{MHz}, \mathrm{CDCl}_{3}\right)$

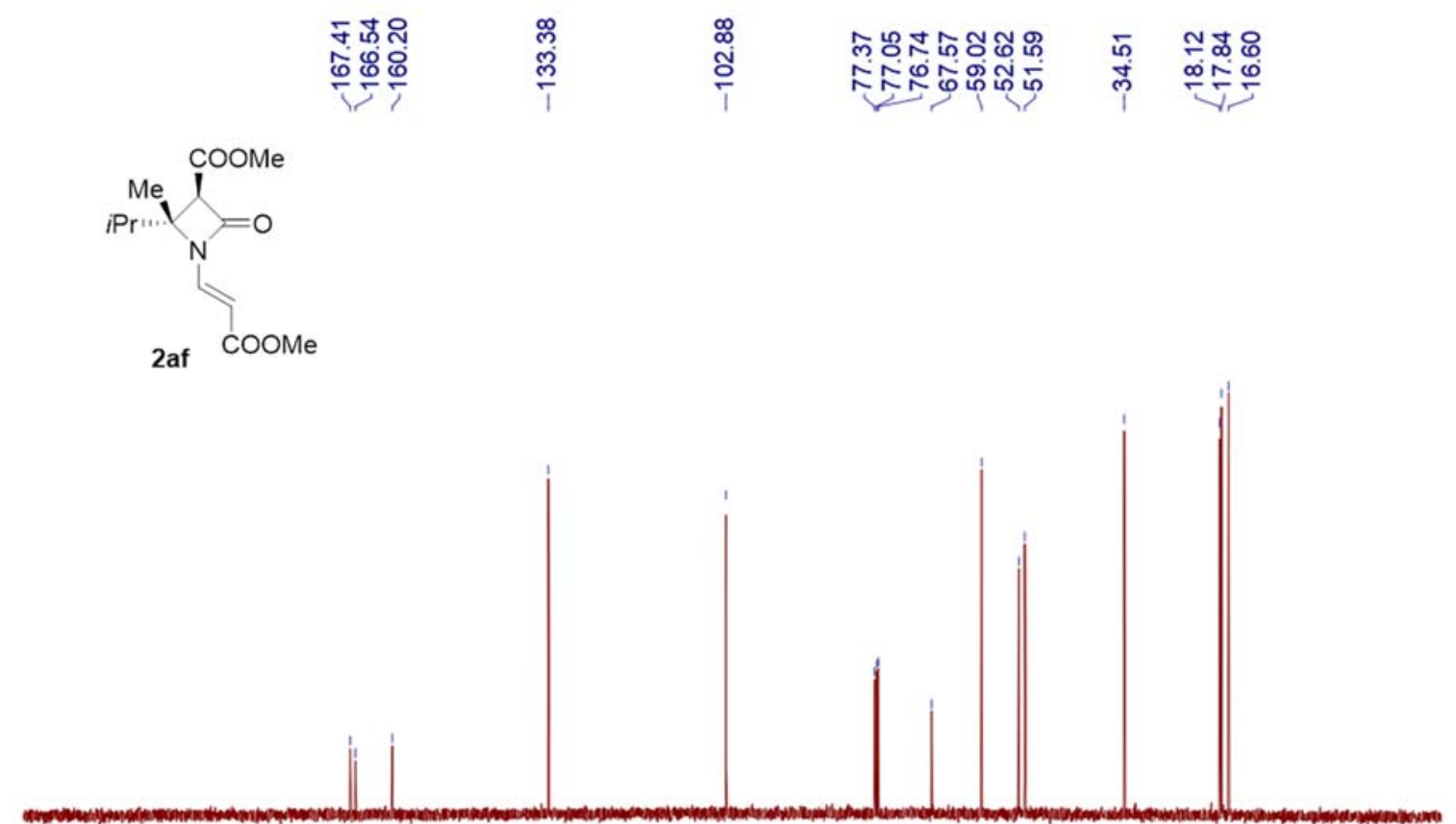



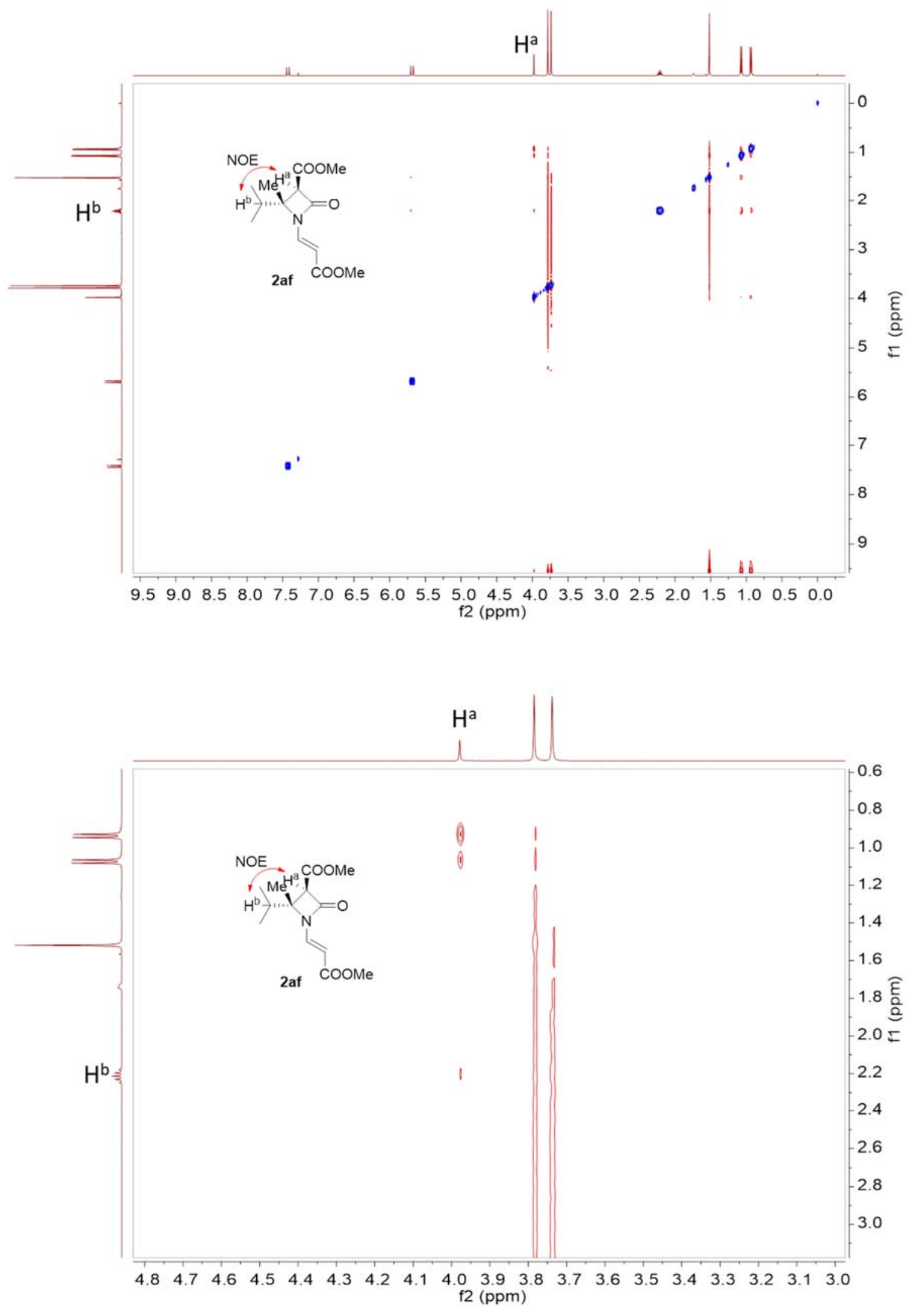
${ }^{1} \mathrm{H}$ NMR of $2 \mathbf{a g}\left(400 \mathrm{MHz}, \mathrm{CDCl}_{3}\right)$

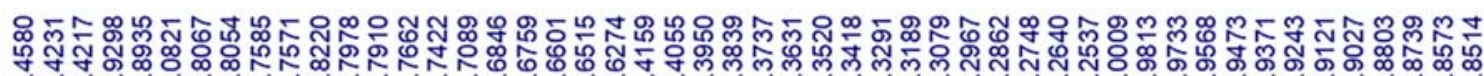

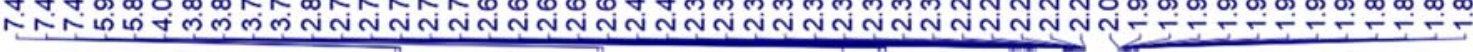

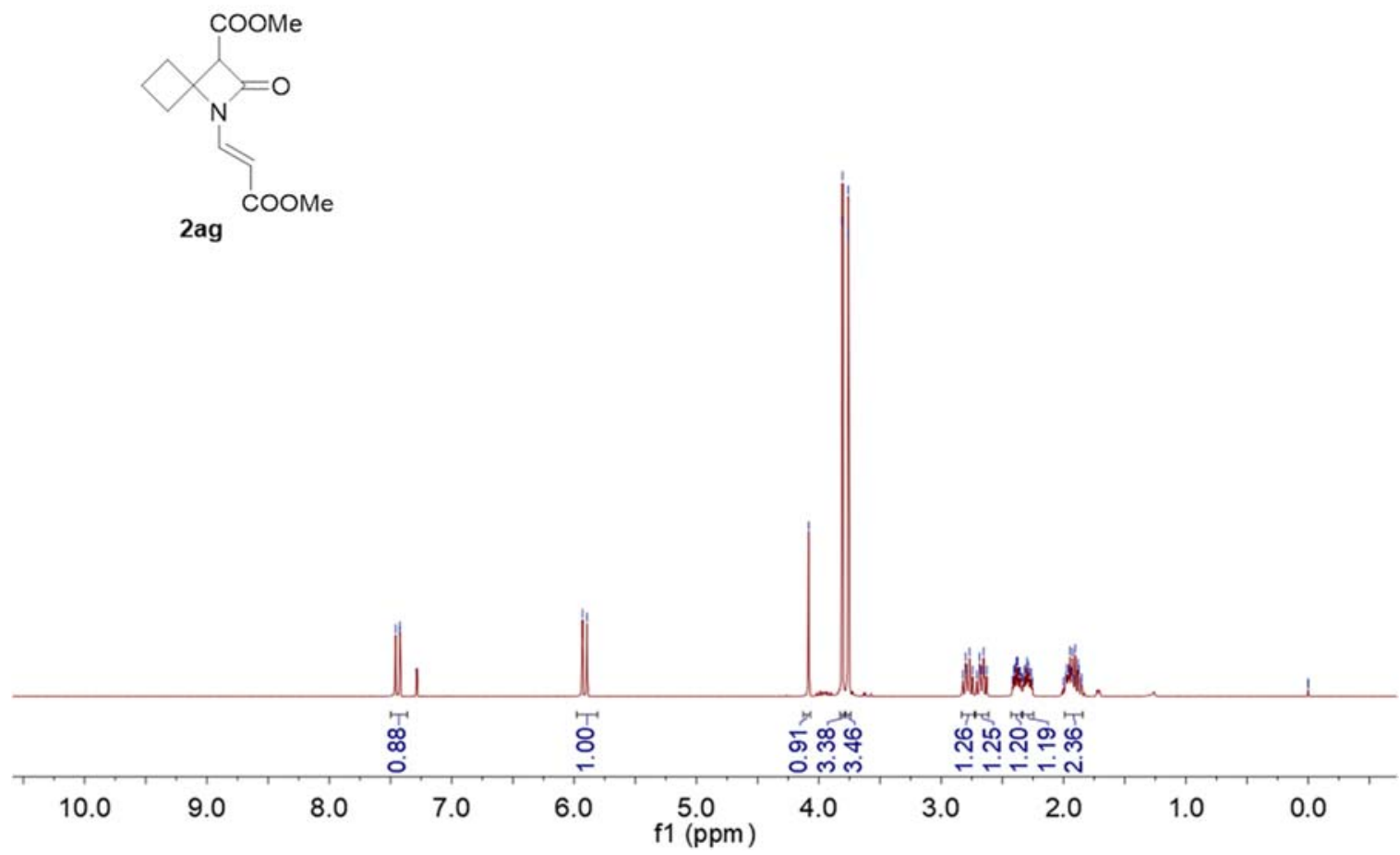

${ }^{13} \mathrm{C} \mathrm{NMR}$ of $2 \mathbf{a g}\left(100 \mathrm{MHz}, \mathrm{CDCl}_{3}\right)$

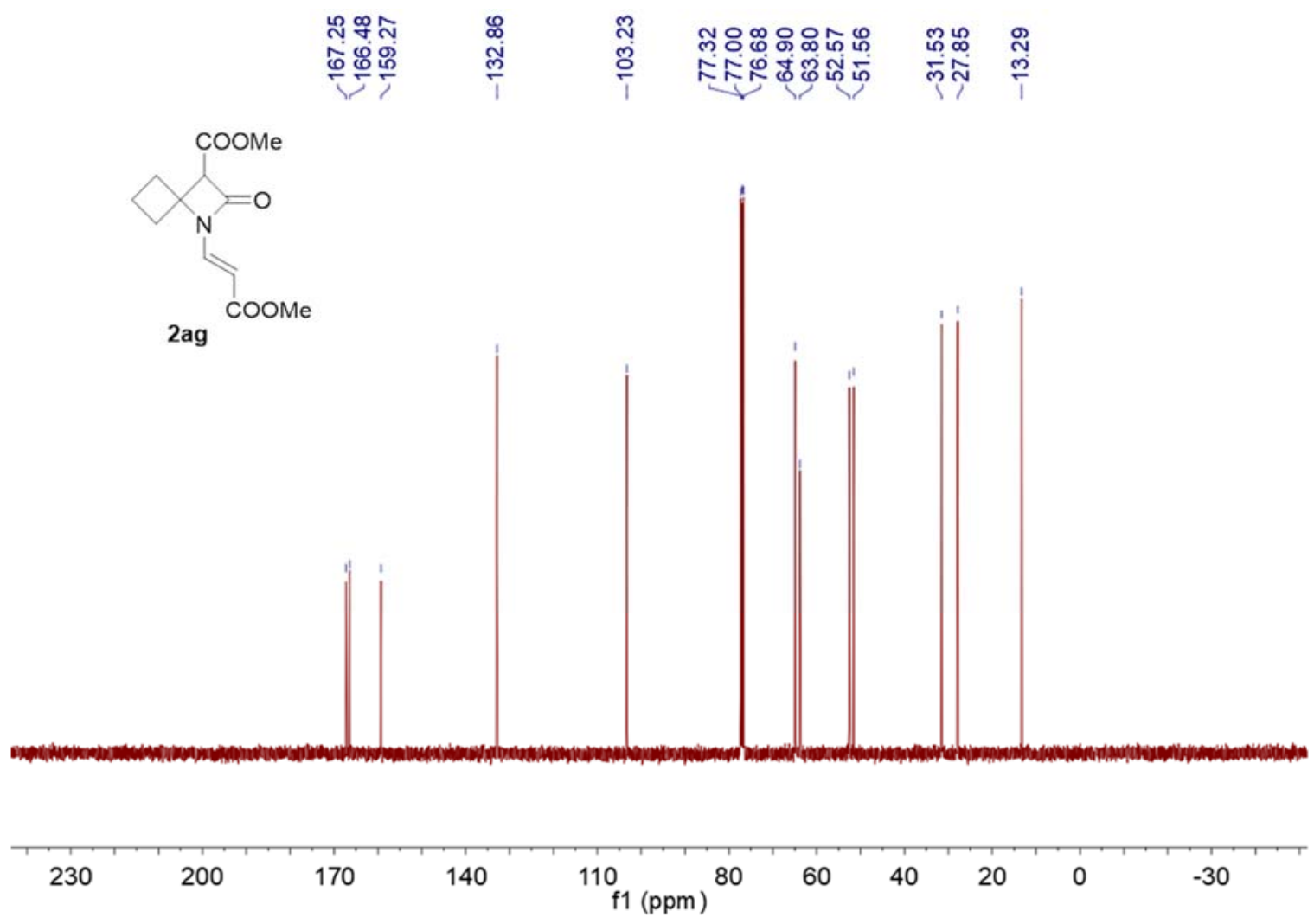


${ }^{1} \mathrm{H}$ NMR of $\mathbf{2 a h}\left(400 \mathrm{MHz}, \mathrm{CDCl}_{3}\right)$

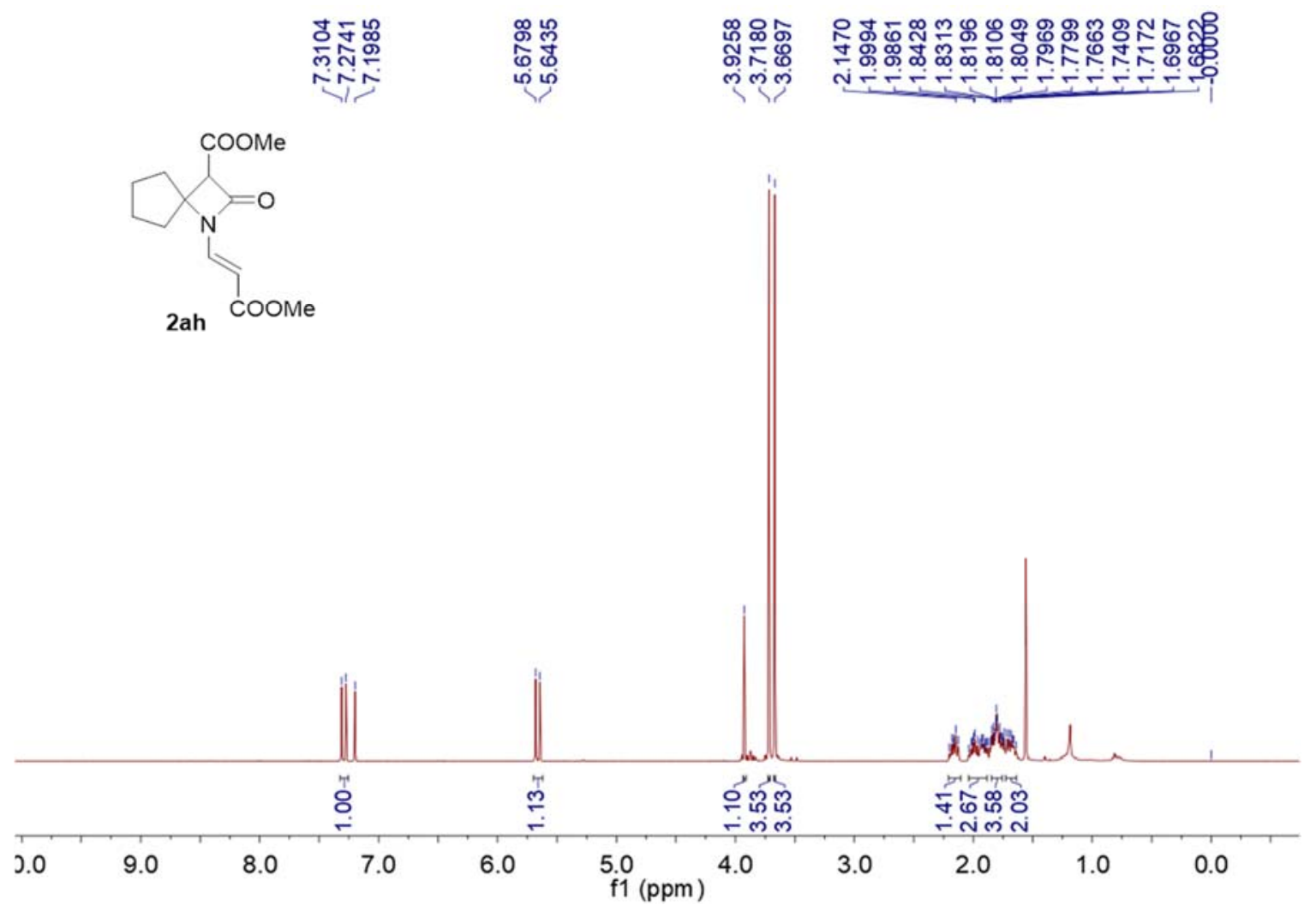

${ }^{13} \mathrm{C}$ NMR of 2ah $\left(100 \mathrm{MHz}, \mathrm{CDCl}_{3}\right)$

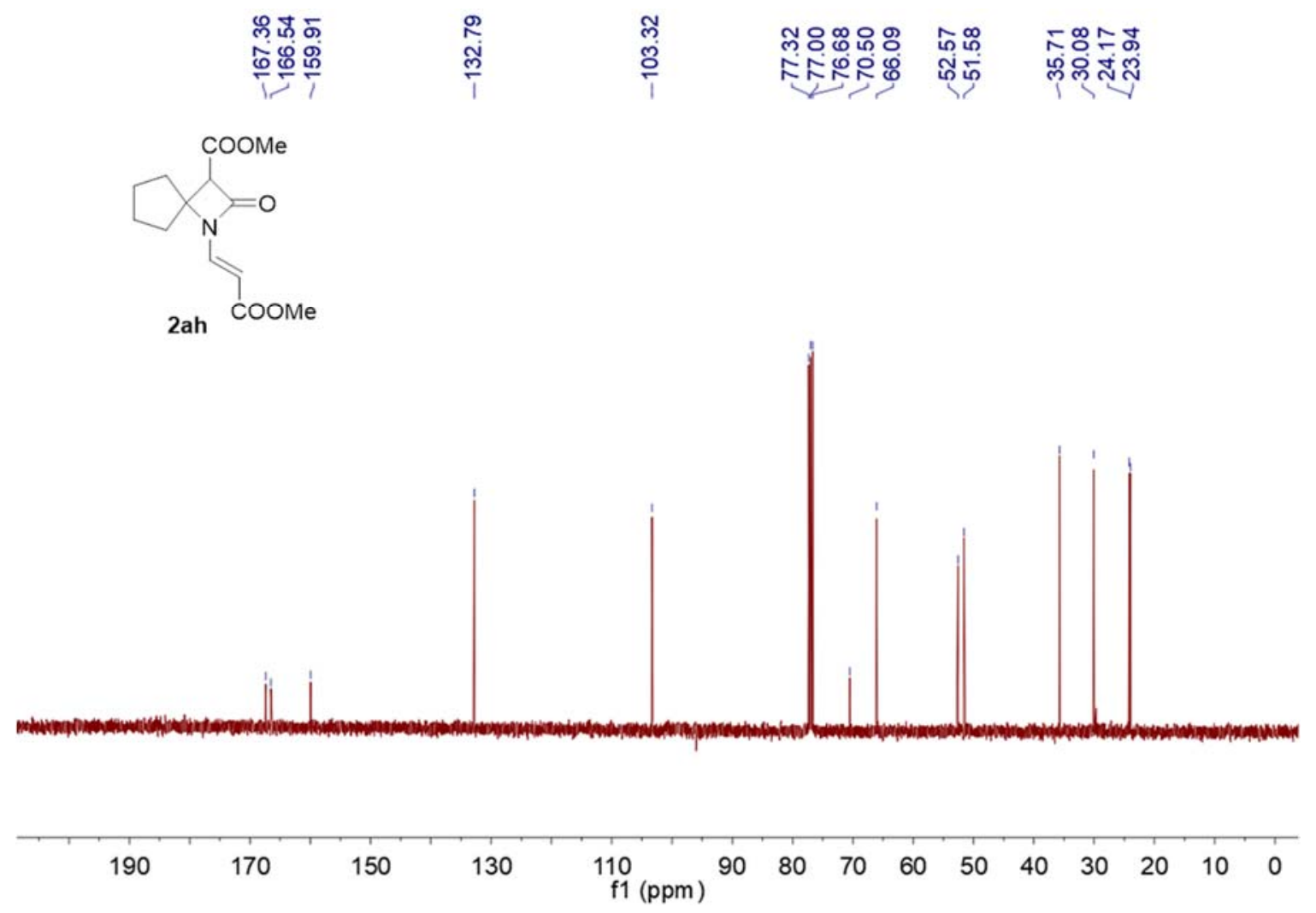


${ }^{1} \mathrm{H}$ NMR of 2ai $\left(400 \mathrm{MHz}, \mathrm{CDCl}_{3}\right)$

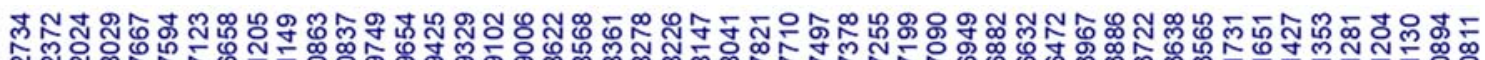
N NָN

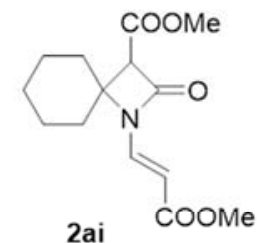

$2 a i$
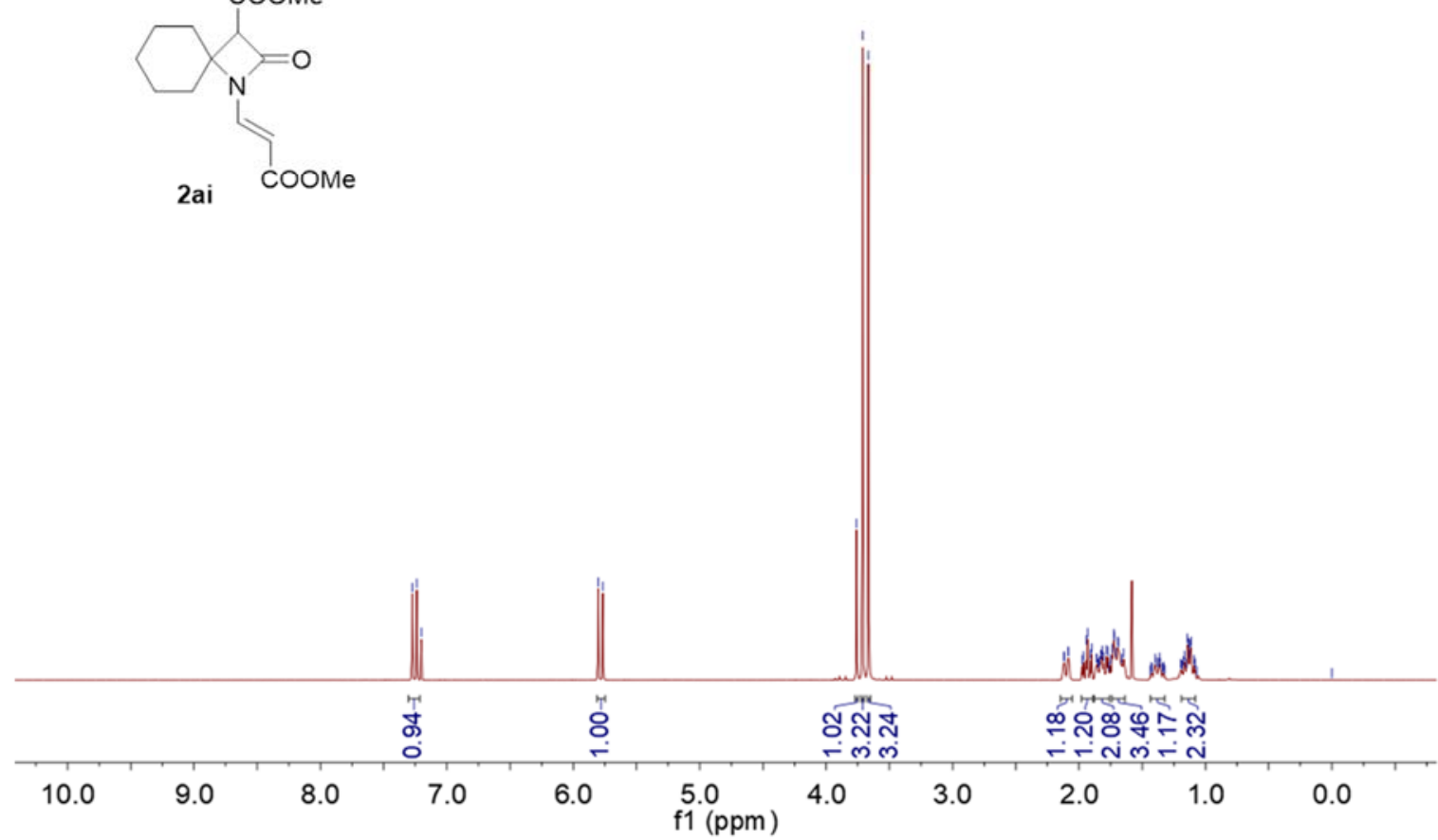

${ }^{13} \mathrm{C}$ NMR of 2ai $\left(100 \mathrm{MHz}, \mathrm{CDCl}_{3}\right)$
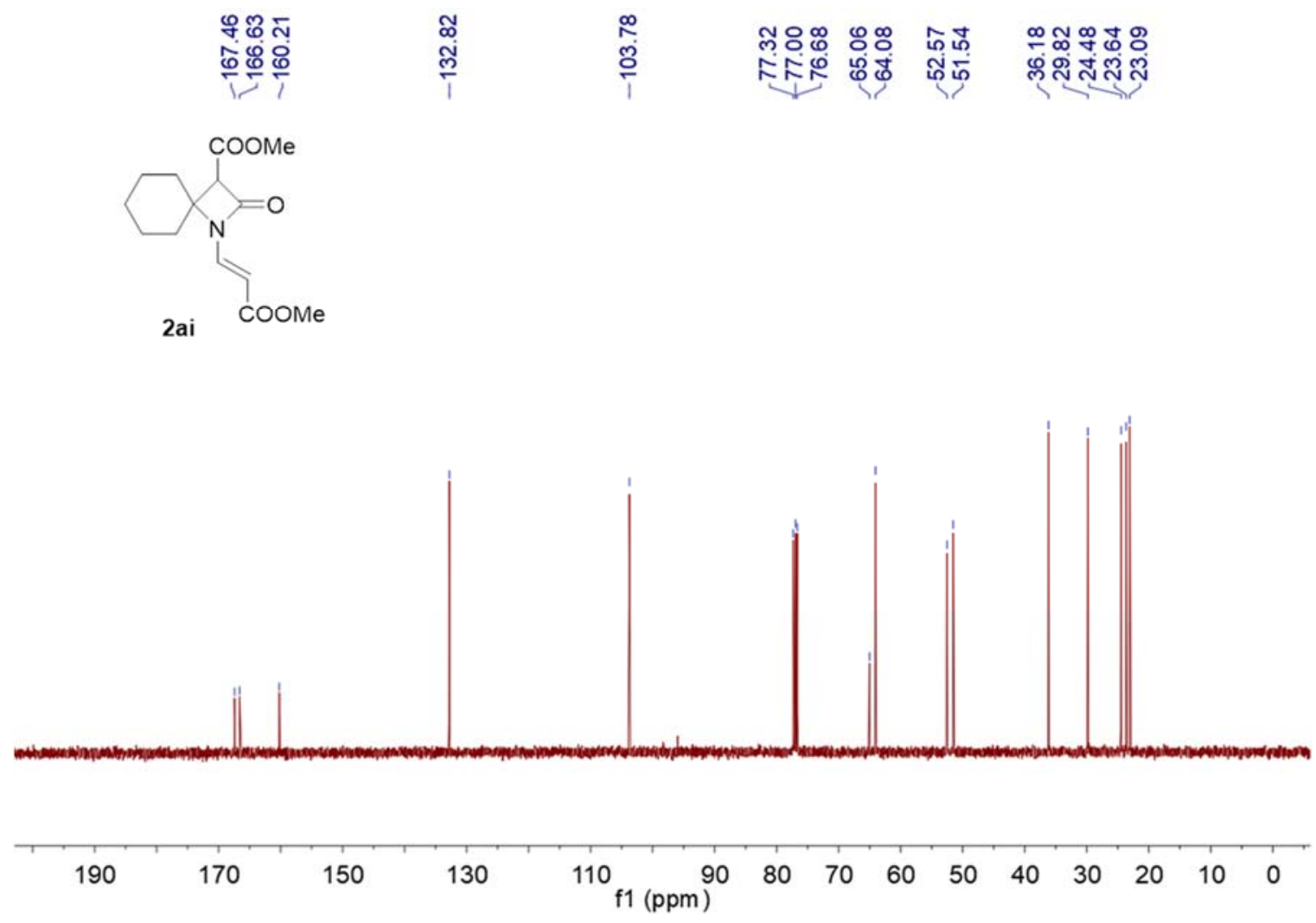
${ }^{1} \mathrm{H}$ NMR of $\mathbf{2 a j}\left(400 \mathrm{MHz}, \mathrm{CDCl}_{3}\right)$

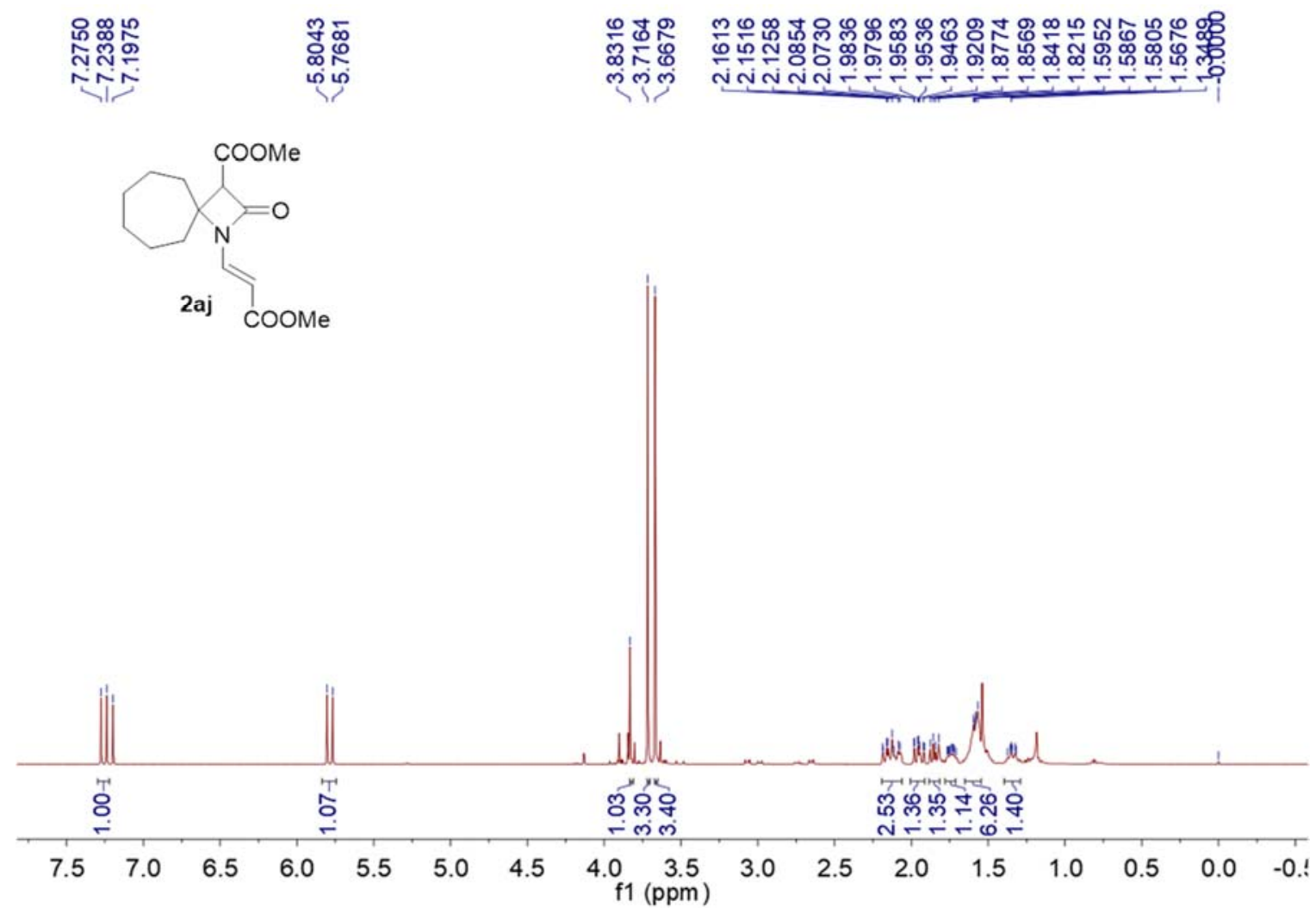

${ }^{13} \mathrm{C}$ NMR of 2aj $\left(100 \mathrm{MHz}, \mathrm{CDCl}_{3}\right)$

\begin{tabular}{|c|c|c|c|c|}
\hline 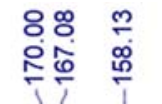 & ్ָ & 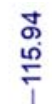 & 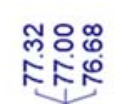 & 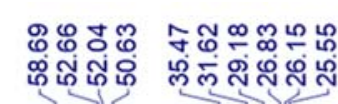 \\
\hline
\end{tabular}
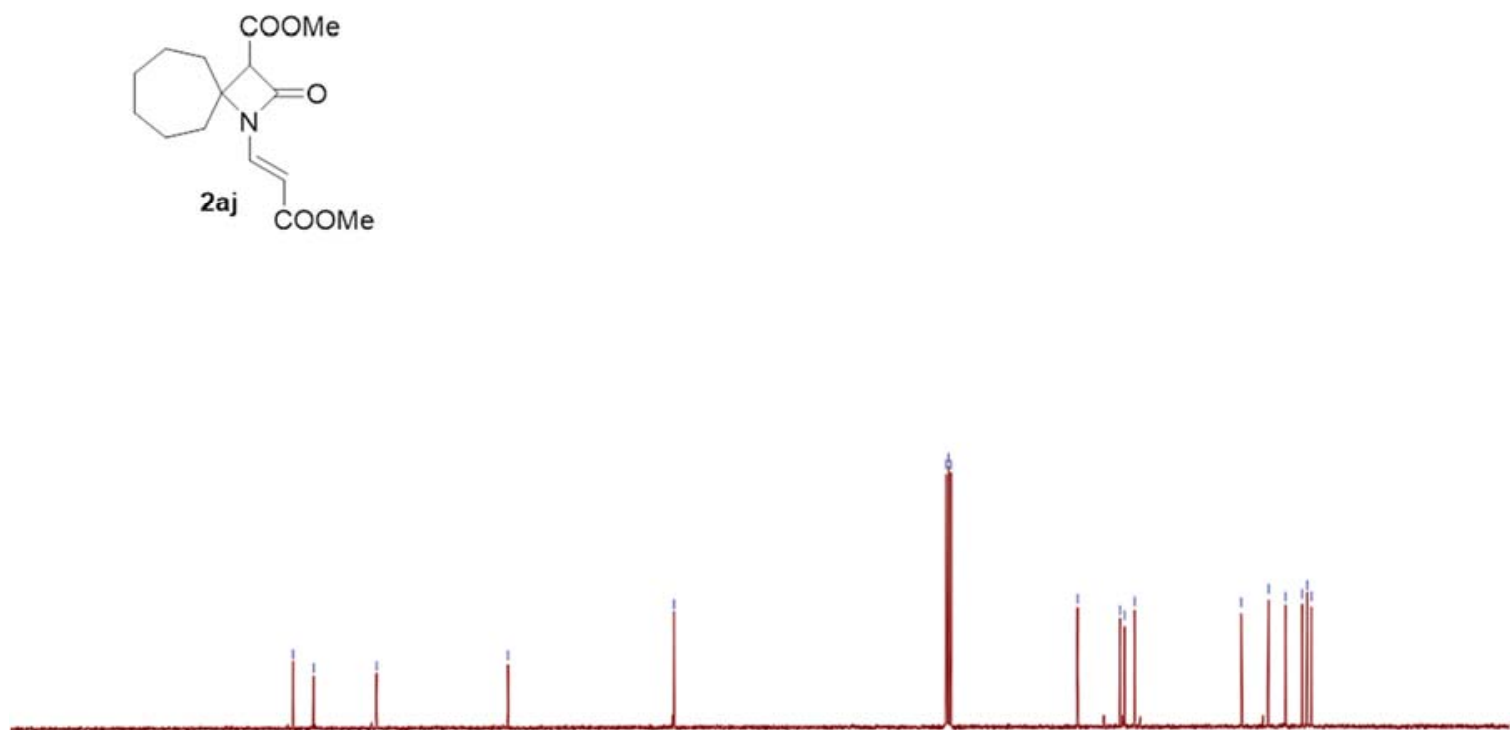

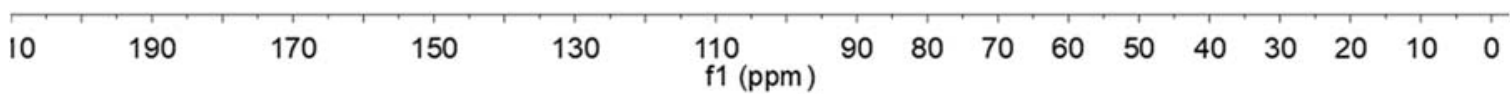


${ }^{1} \mathrm{H}$ NMR of $\mathbf{2 a k}\left(400 \mathrm{MHz}, \mathrm{CDCl}_{3}\right)$

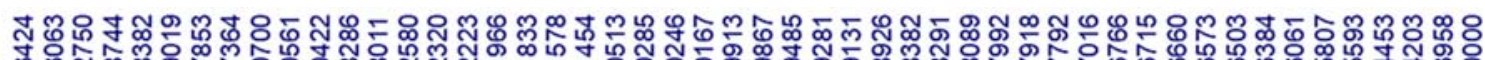

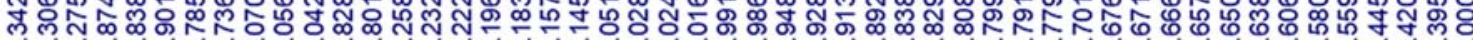

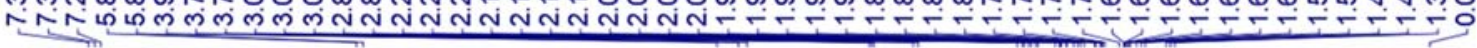<smiles>COC(=O)/C=C/N1C(=O)C(C(C)=O)C12CCCCCCC2</smiles>

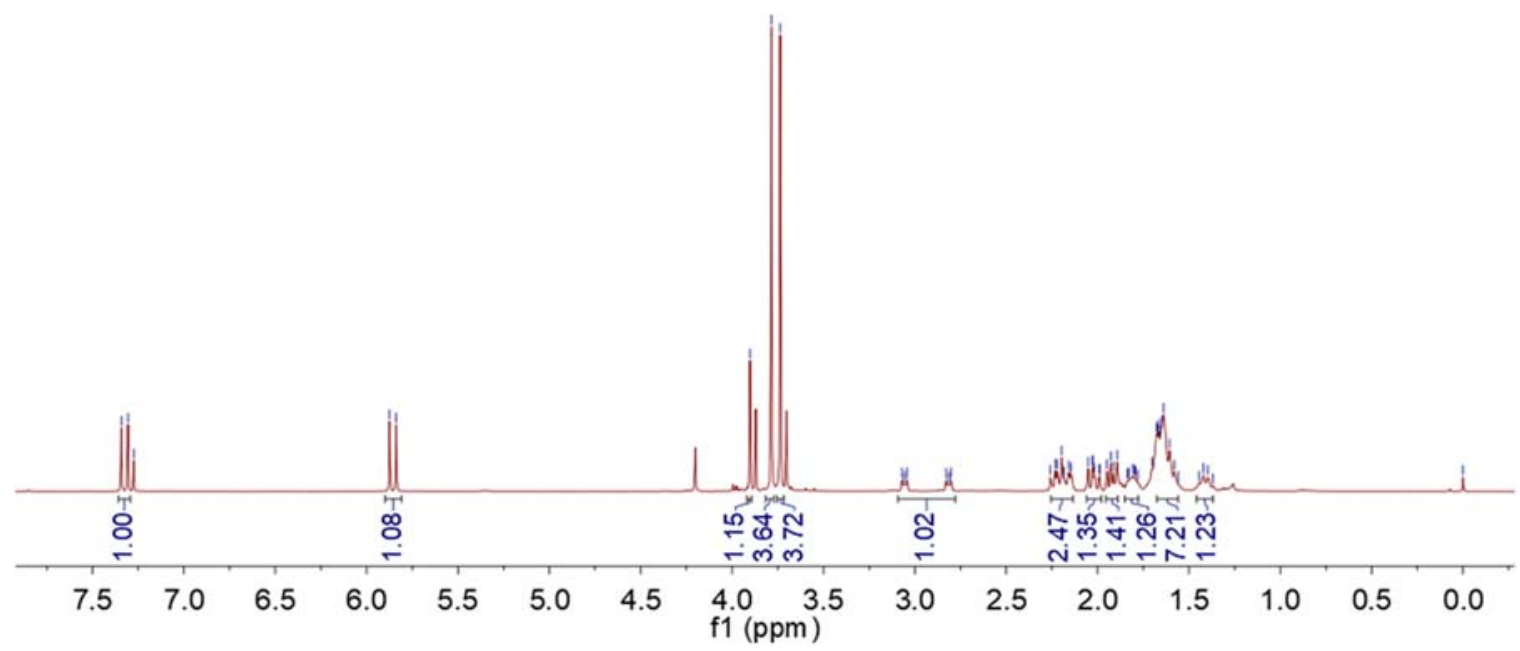

${ }^{13} \mathrm{C}$ NMR of 2ak (100 MHz, $\mathrm{CDCl}_{3}$ )

\begin{tabular}{|c|c|c|c|c|}
\hline 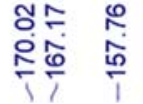 & $\begin{array}{l}\bar{y} \\
\stackrel{e}{+}\end{array}$ & $\stackrel{\bar{N}}{\stackrel{N}{\leftarrow}}$ & 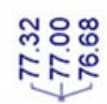 & 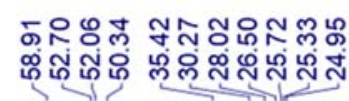 \\
\hline
\end{tabular}<smiles>COC(=O)C=CN1C(=O)C(C(C)=O)C12CCCCCCC2</smiles>

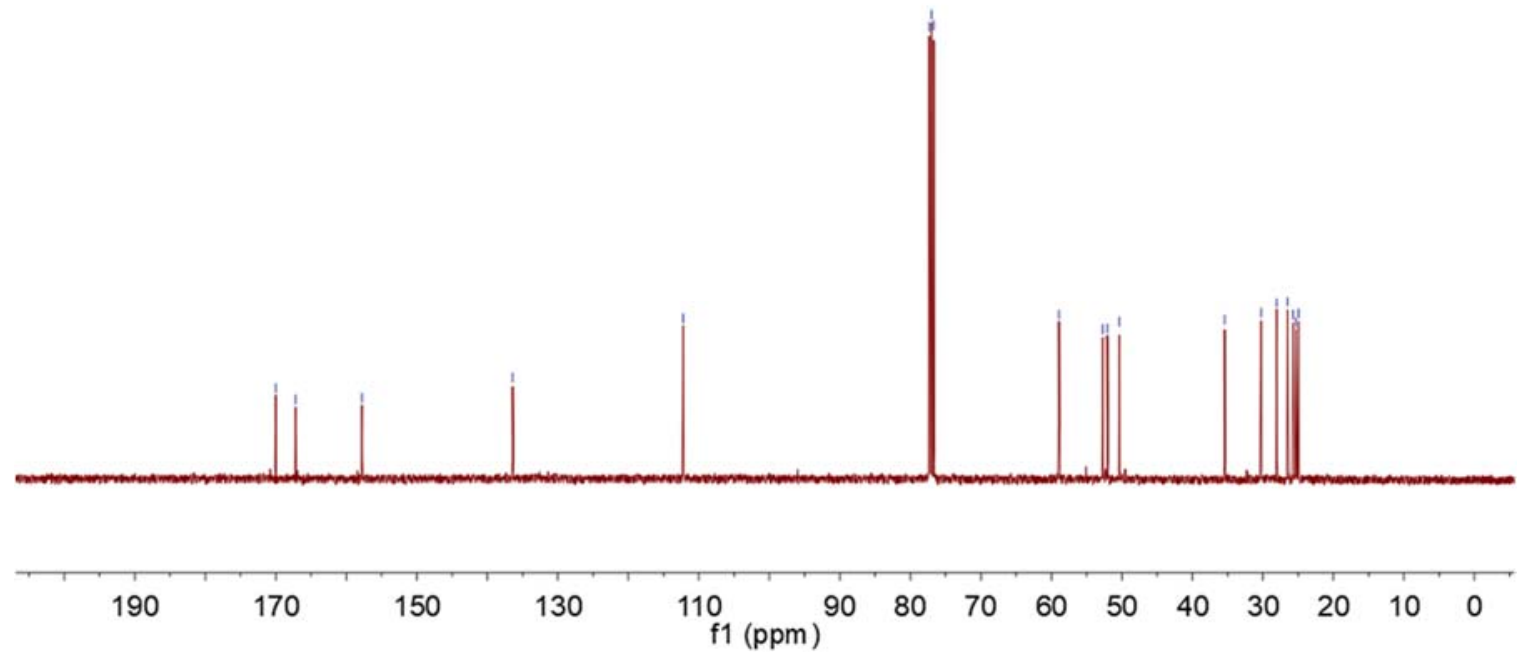


${ }^{1} \mathrm{H} \mathrm{NMR}$ of 2 al $\left(400 \mathrm{MHz}, \mathrm{CDCl}_{3}\right)$
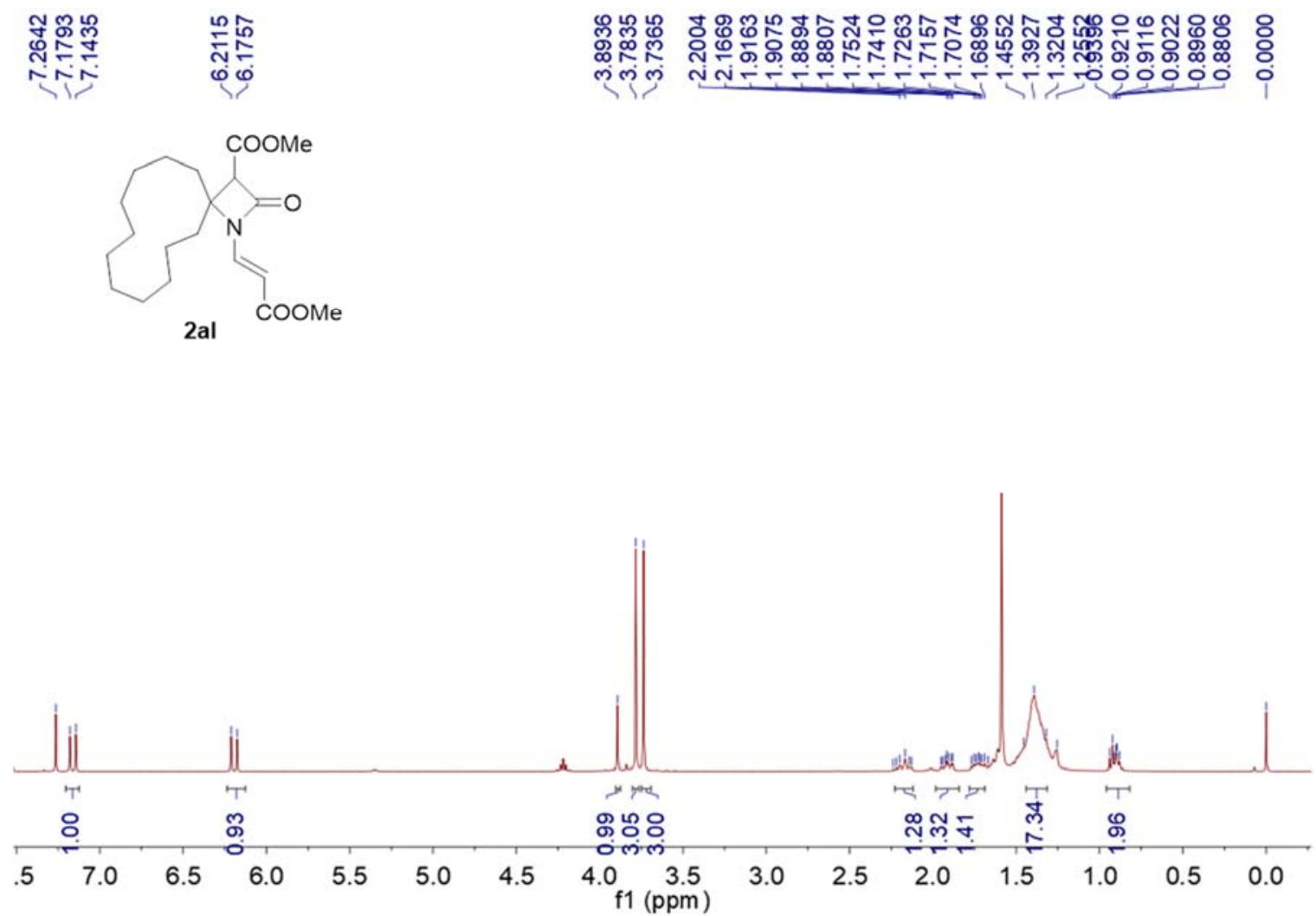

${ }^{13} \mathrm{C}$ NMR of 2 al $\left(100 \mathrm{MHz}, \mathrm{CDCl}_{3}\right)$

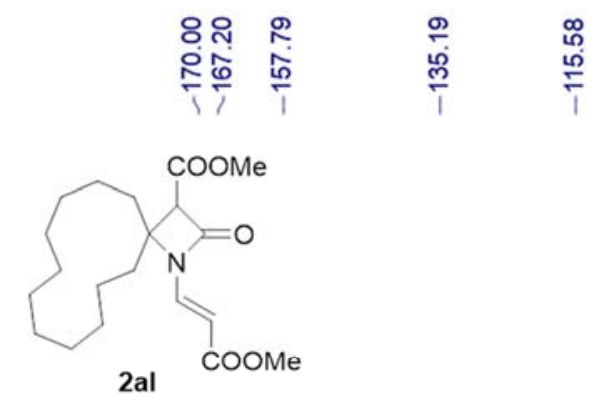

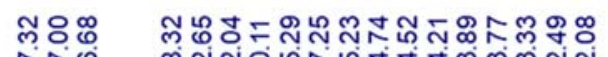

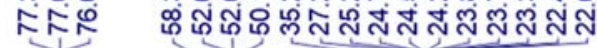

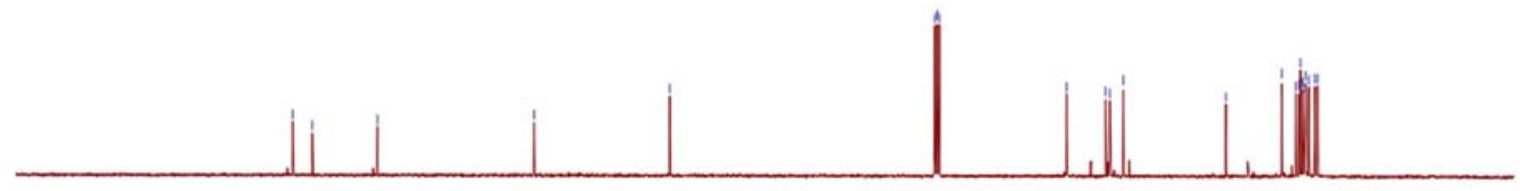

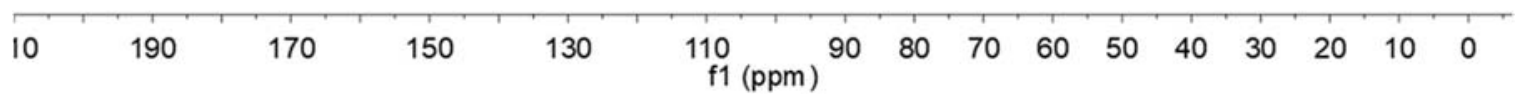


${ }^{1} \mathrm{H}$ NMR of $2 \mathbf{a m}\left(400 \mathrm{MHz}, \mathrm{CDCl}_{3}\right)$

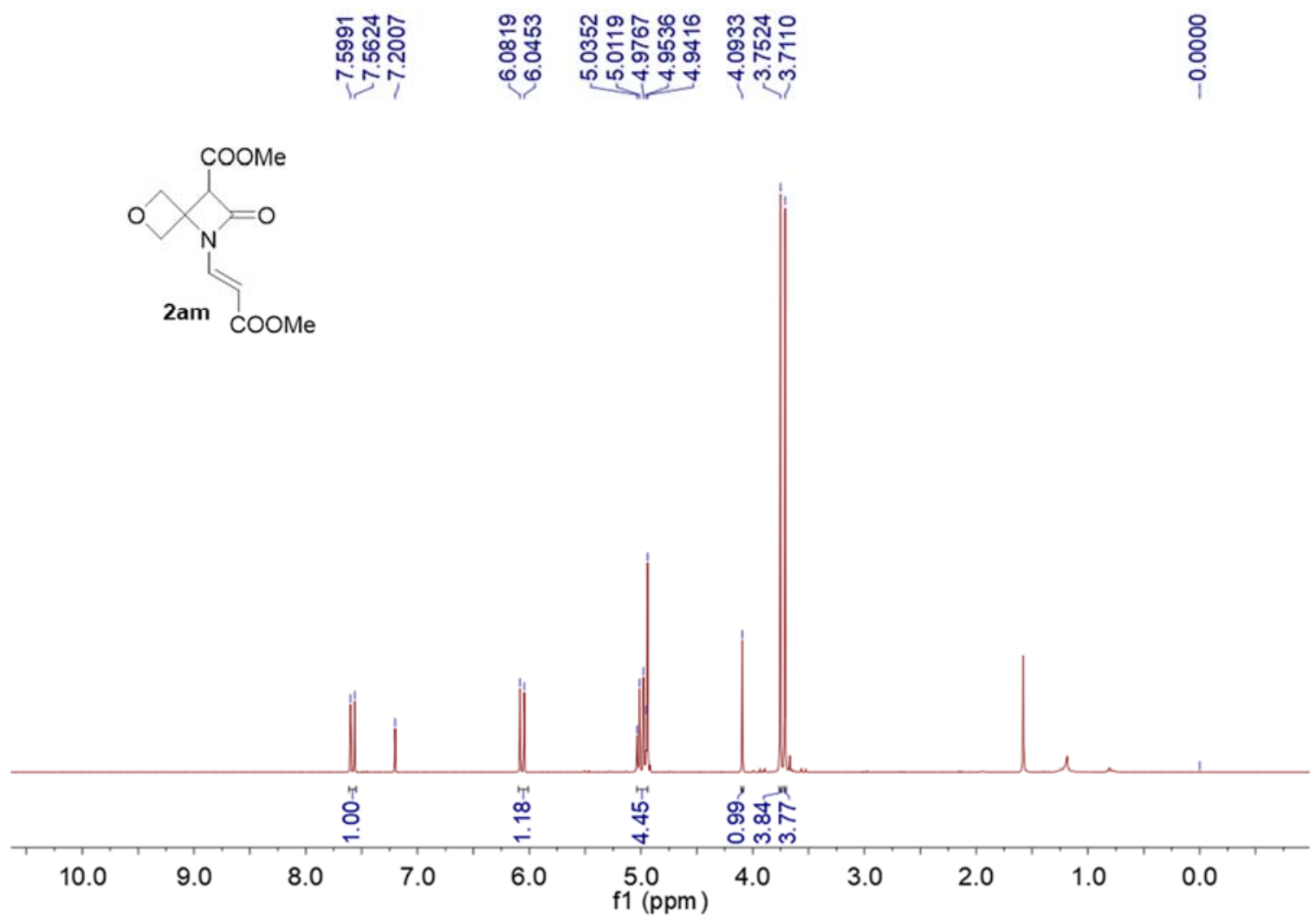

${ }^{13} \mathrm{C}$ NMR of $2 \mathbf{a m}\left(100 \mathrm{MHz}, \mathrm{CDCl}_{3}\right)$

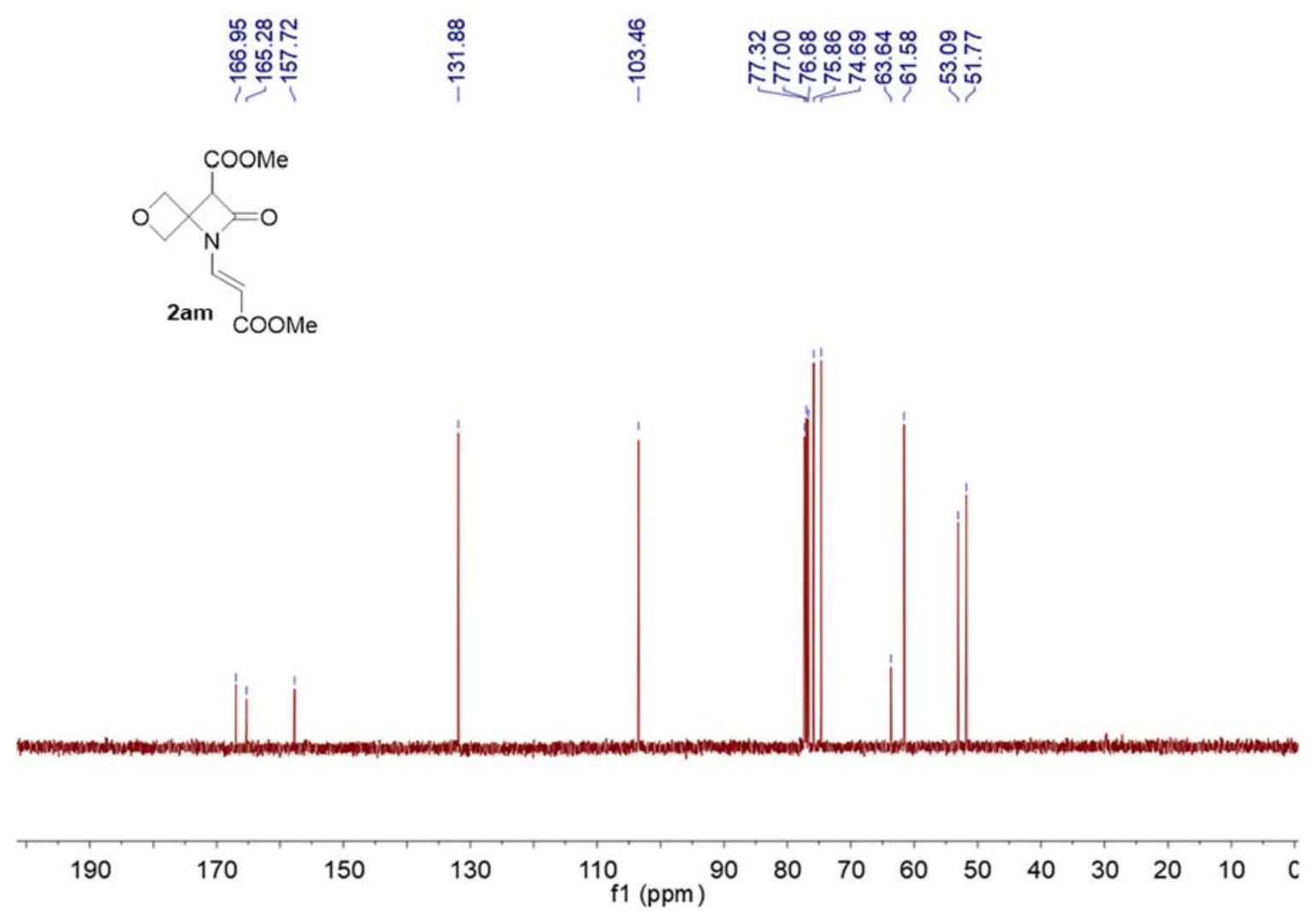


${ }^{1} \mathrm{H}$ NMR of $2 \mathbf{a n}\left(400 \mathrm{MHz}, \mathrm{CDCl}_{3}\right)$

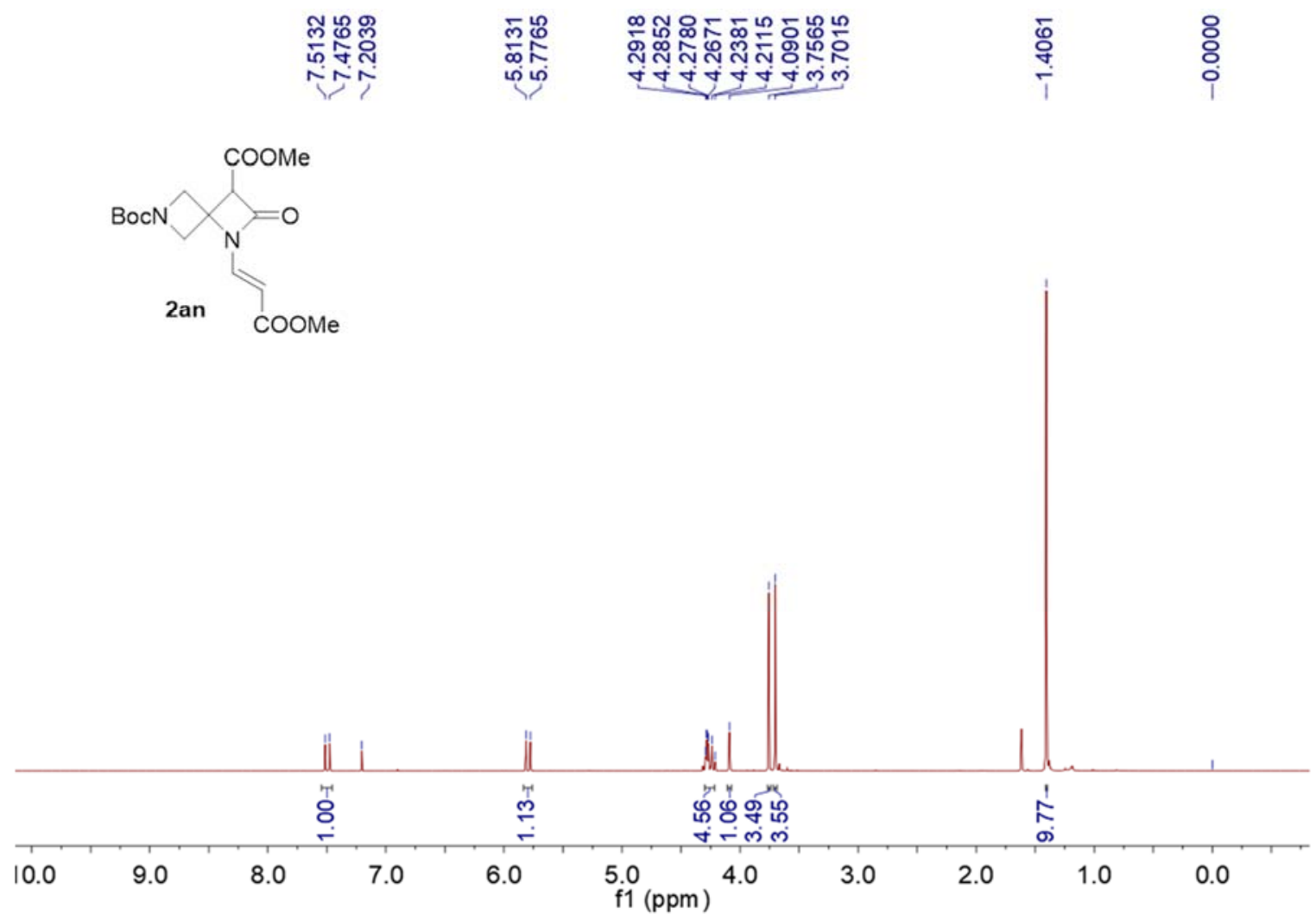

${ }^{13} \mathrm{C}$ NMR of 2 an $\left(100 \mathrm{MHz}, \mathrm{CDCl}_{3}\right)$

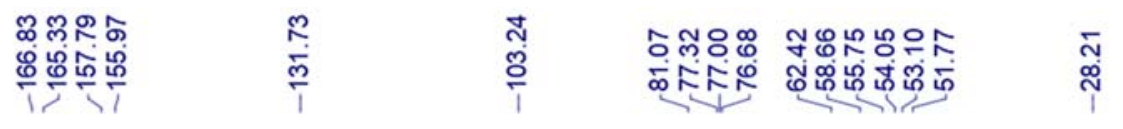<smiles></smiles>

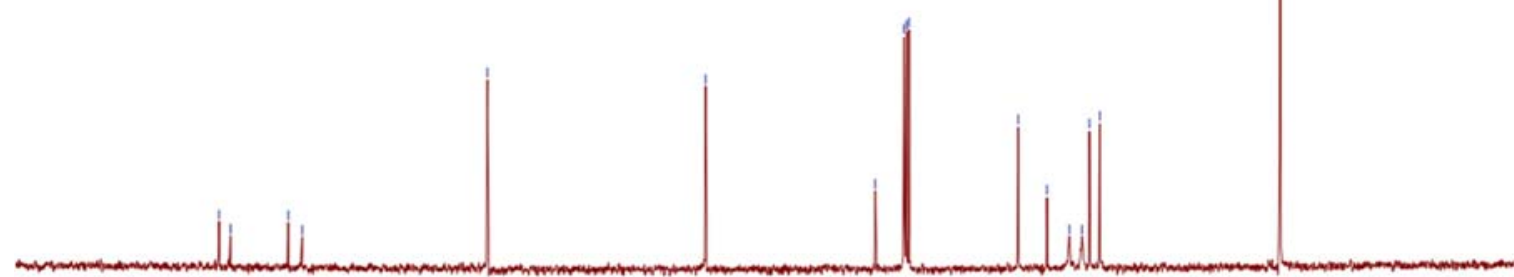

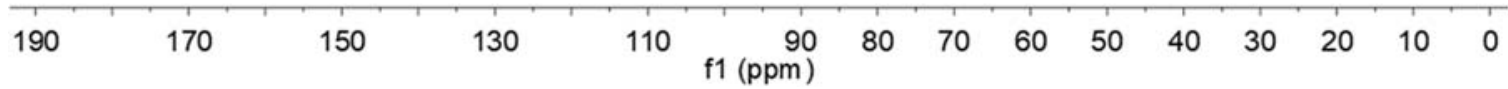


${ }^{1} \mathrm{H}$ NMR of $2 \mathbf{a o}\left(400 \mathrm{MHz}, \mathrm{CDCl}_{3}\right)$

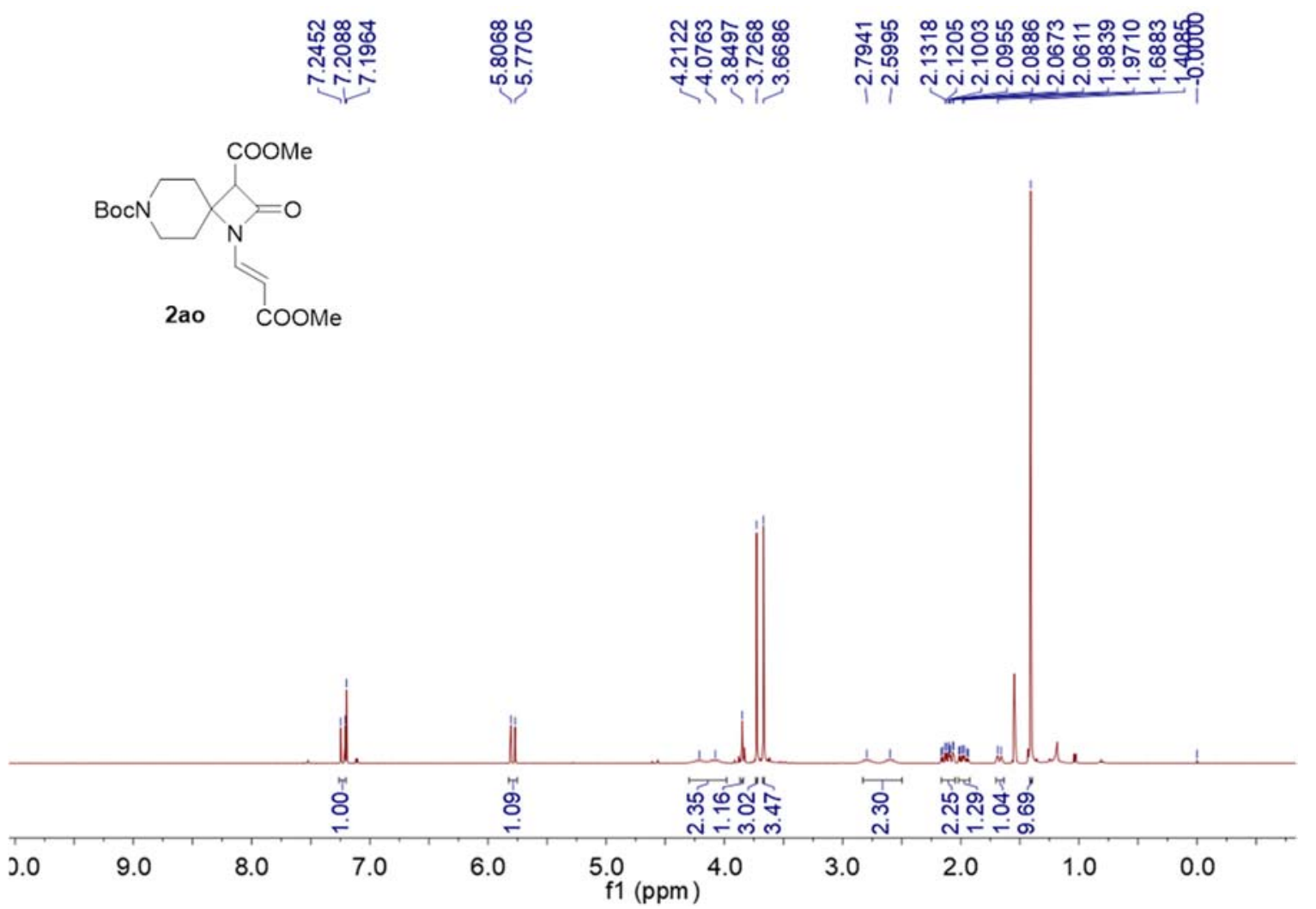

${ }^{13} \mathrm{C}$ NMR of 2 ao $\left(100 \mathrm{MHz}, \mathrm{CDCl}_{3}\right)$

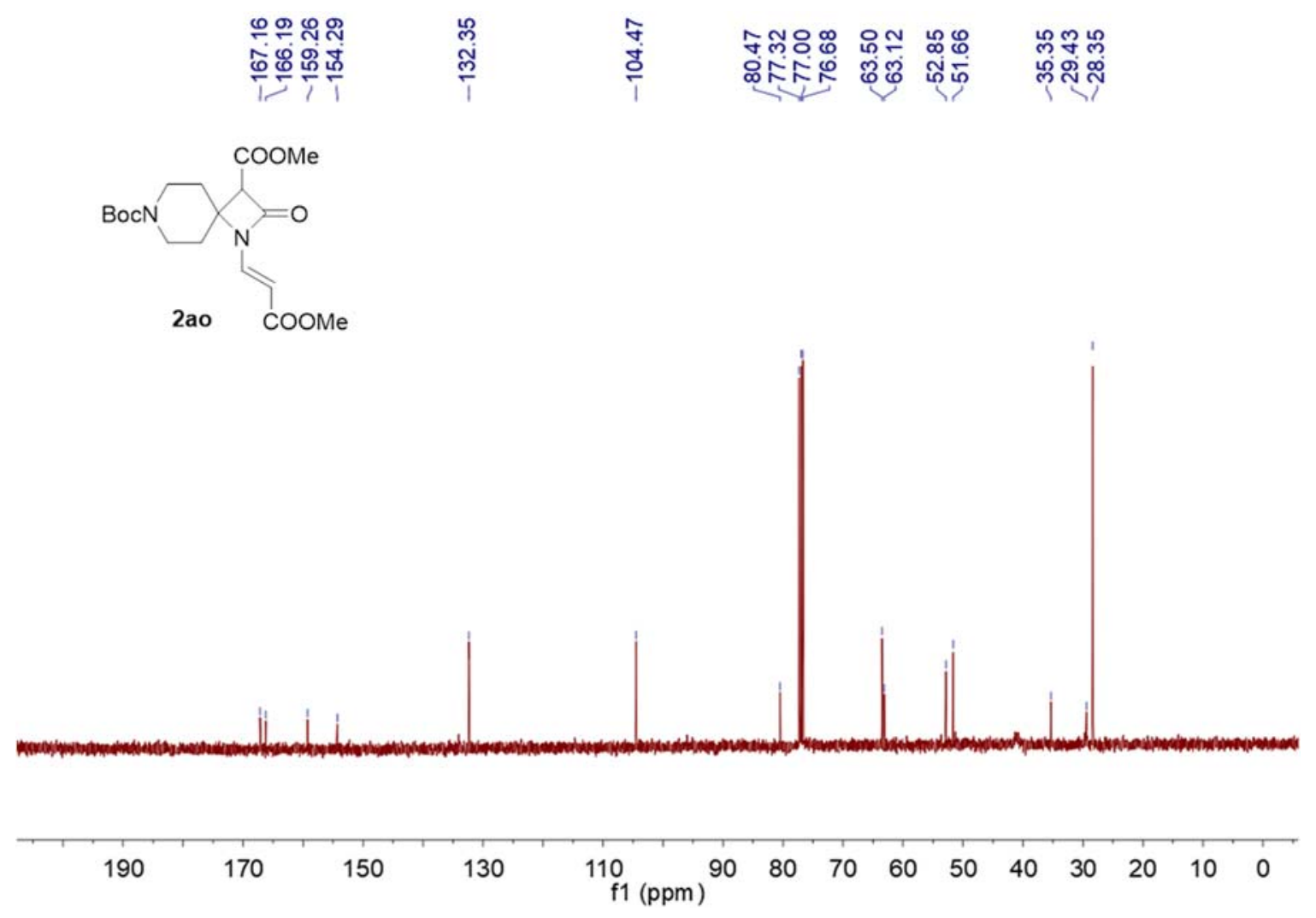


${ }^{1} \mathrm{H}$ NMR of 2ap (400 MHz, $\left.\mathrm{CDCl}_{3}\right)$

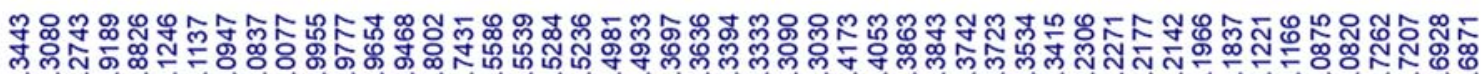

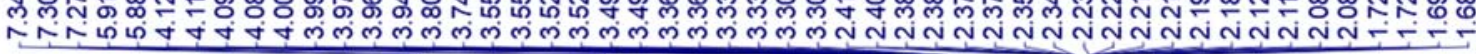

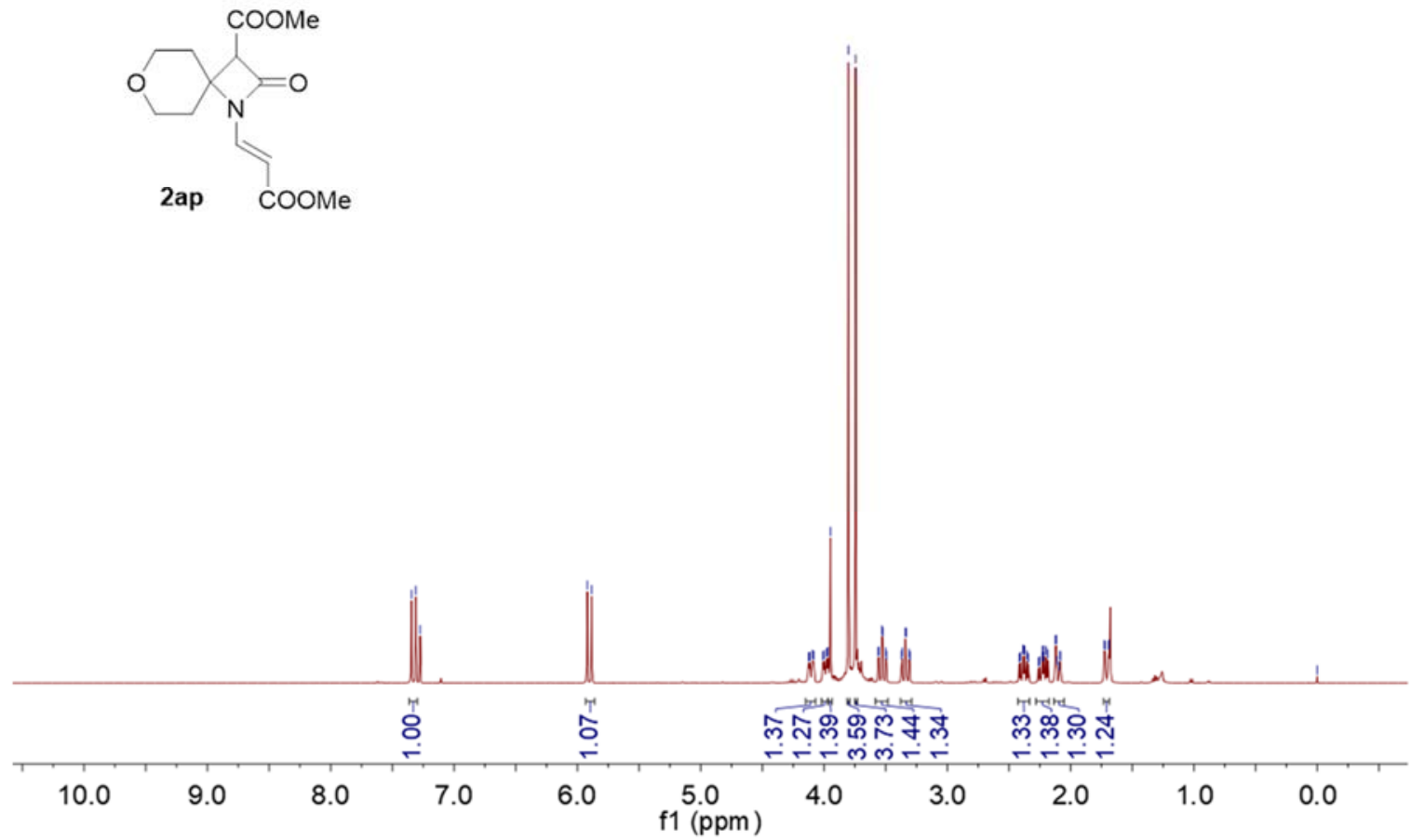

${ }^{13} \mathrm{C}$ NMR of 2 ap $\left(100 \mathrm{MHz}, \mathrm{CDCl}_{3}\right)$

ำกำ

뜨음

$\stackrel{\substack{n \\ \stackrel{m}{1}}}{\stackrel{m}{1}}$

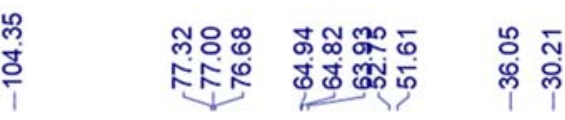
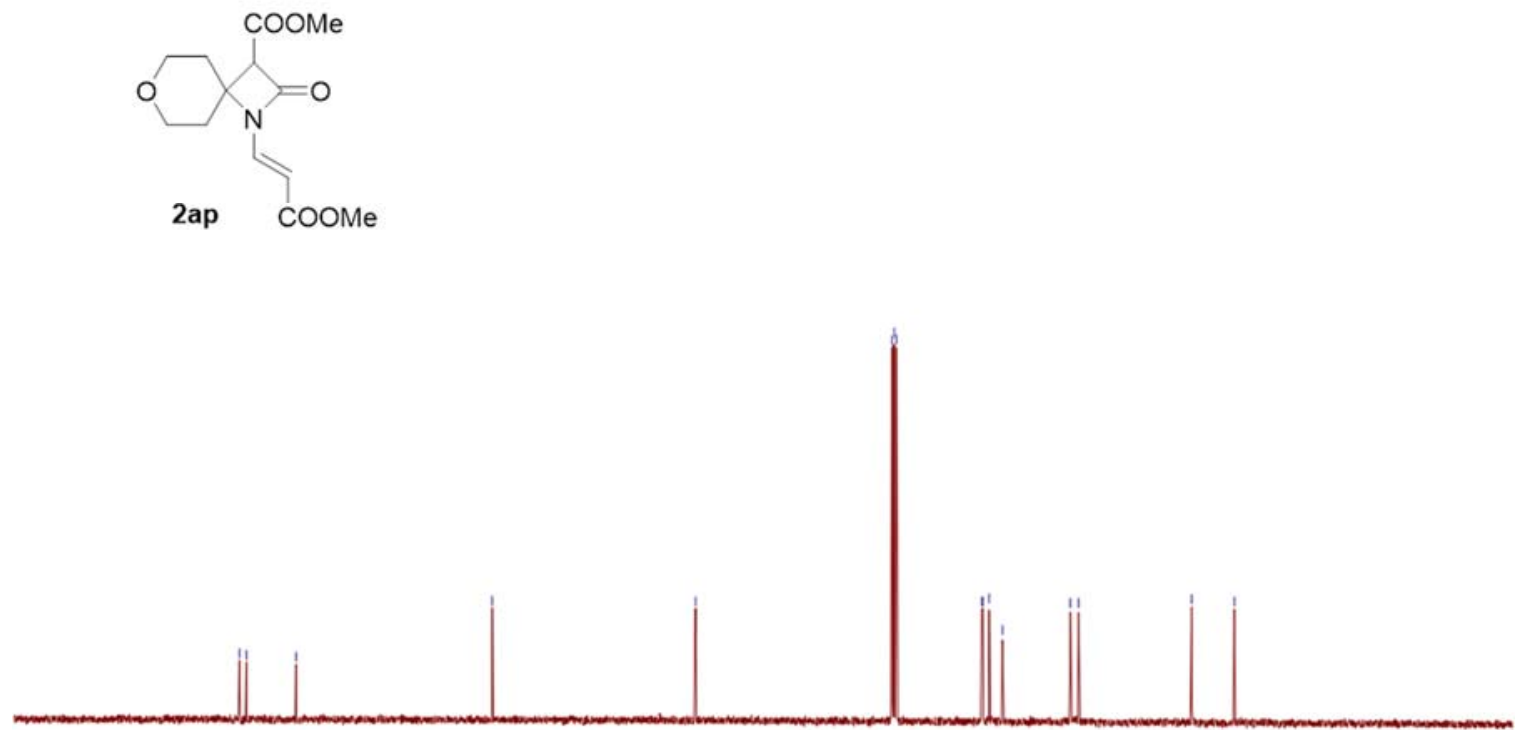

\section{0}

170

150

130

110

f1 (ppm)

$\begin{array}{lllllllll}80 & 70 & 60 & 50 & 40 & 30 & 20 & 10 & 0\end{array}$ 
${ }^{1} \mathrm{H}$ NMR of $\mathbf{2 a q}\left(400 \mathrm{MHz}, \mathrm{CDCl}_{3}\right.$ )

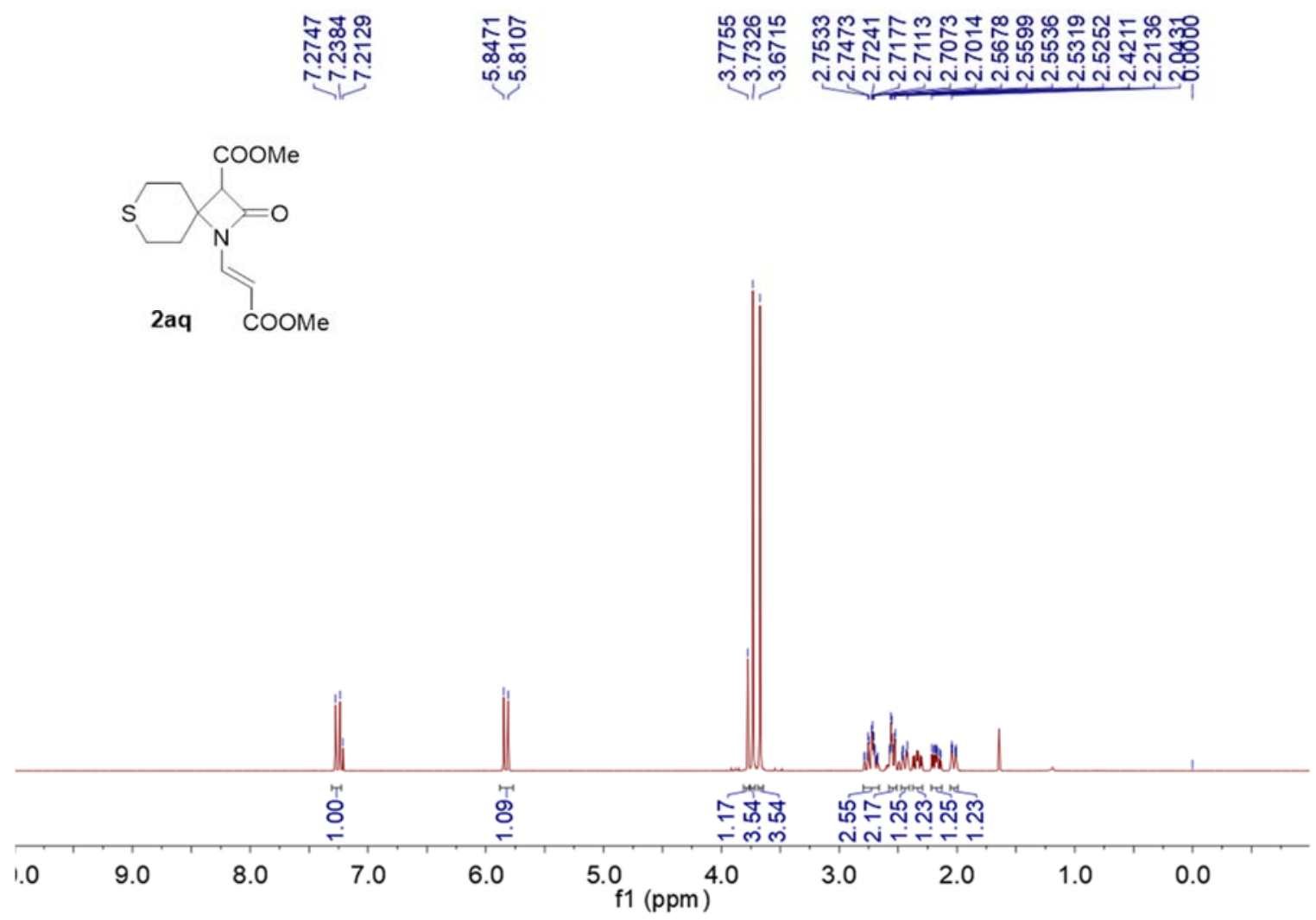

${ }^{13} \mathrm{C}$ NMR of 2 aq $\left(100 \mathrm{MHz}, \mathrm{CDCl}_{3}\right)$

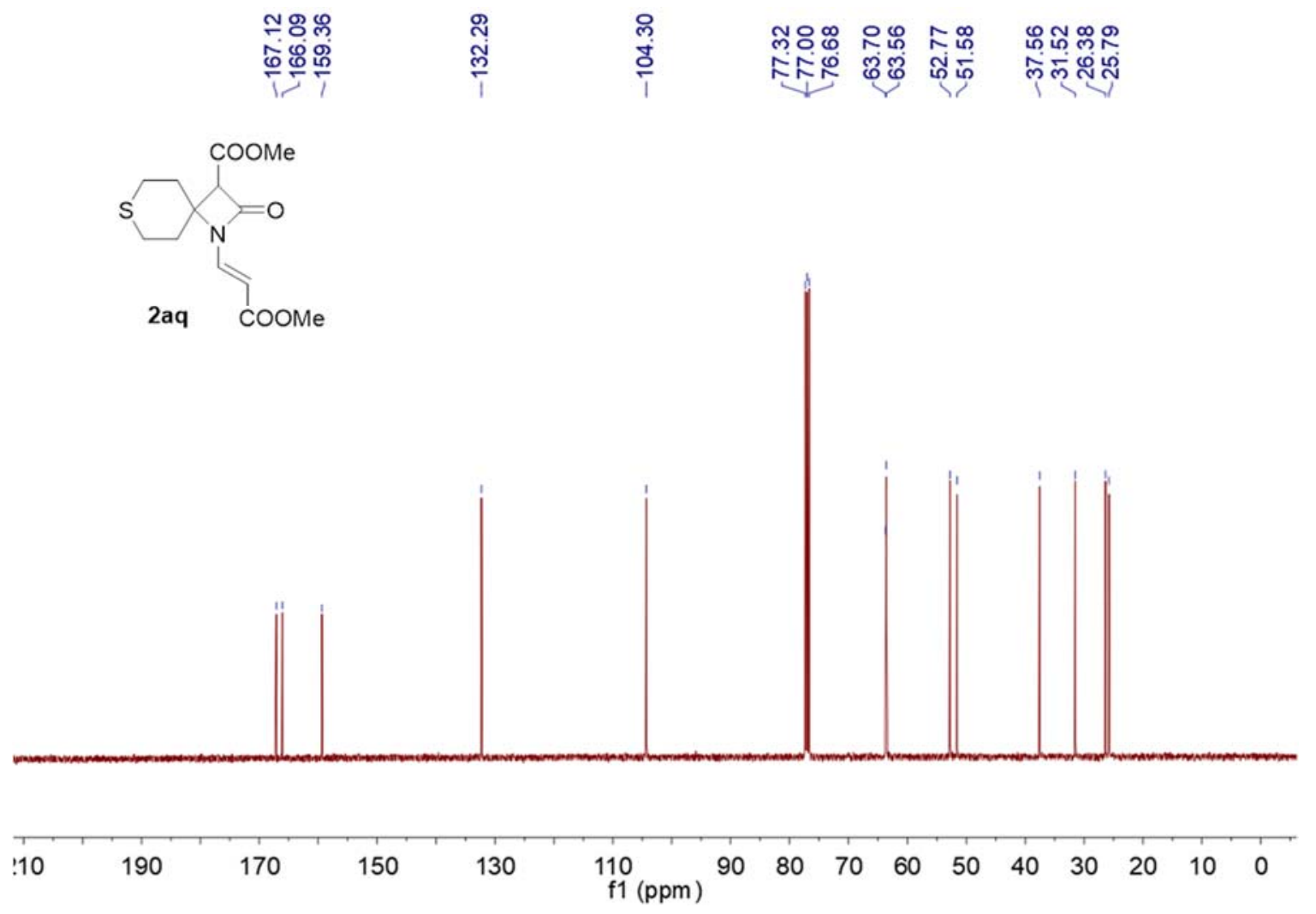


${ }^{1} \mathrm{H}$ NMR of 3 (400 MHz, $\mathrm{CDCl}_{3}$ )

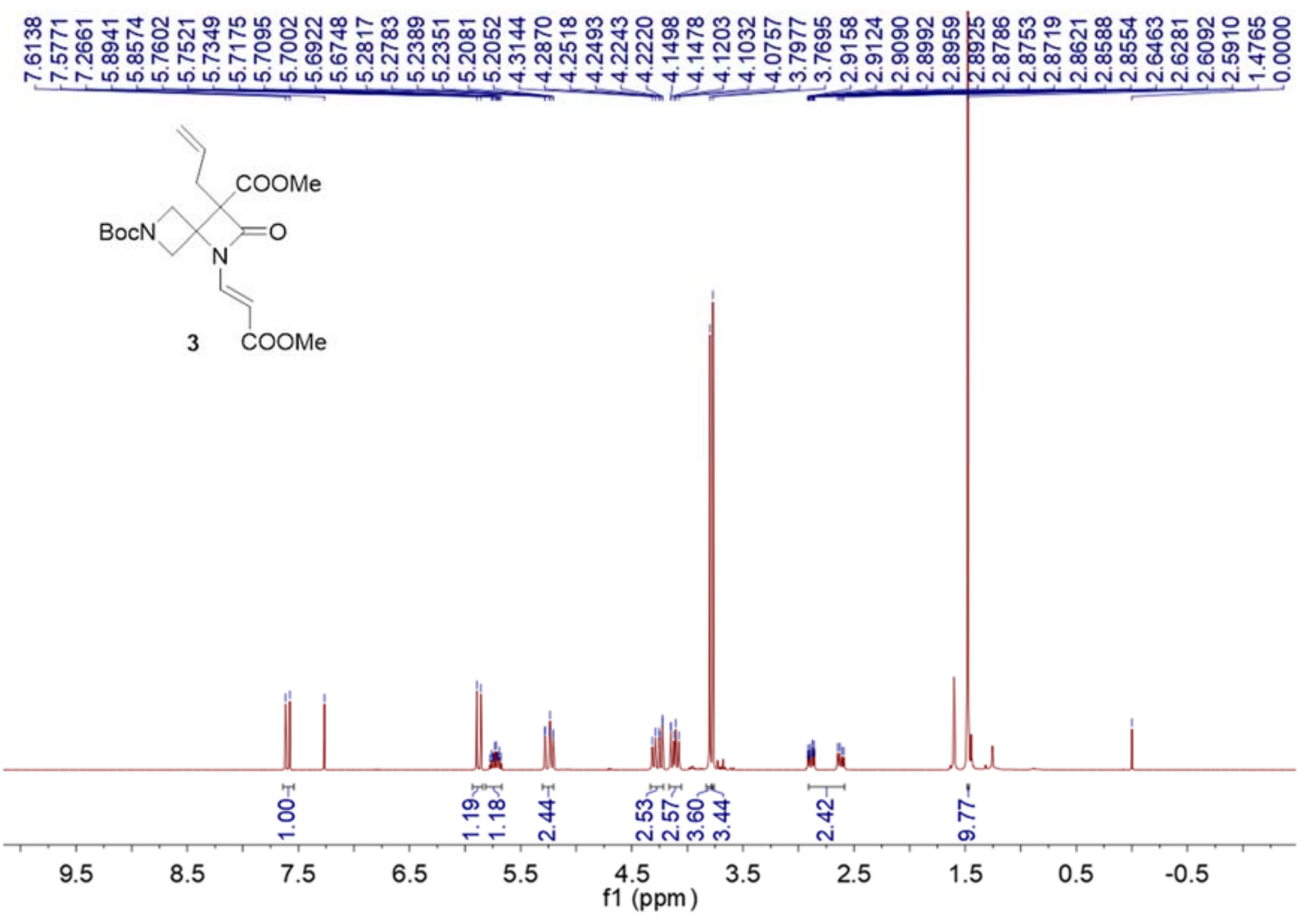

${ }^{13} \mathrm{C}$ NMR of $3\left(100 \mathrm{MHz}, \mathrm{CDCl}_{3}\right)$

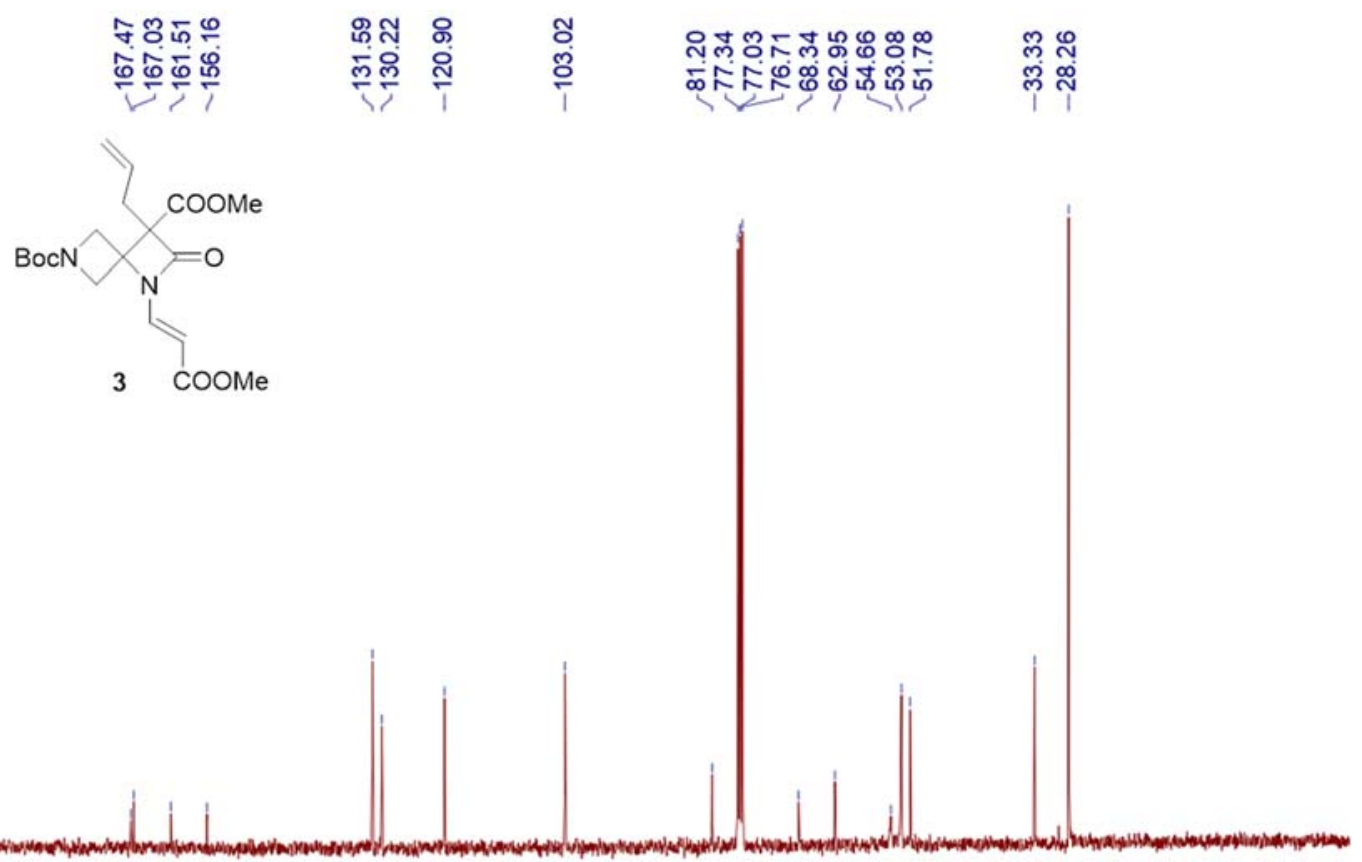

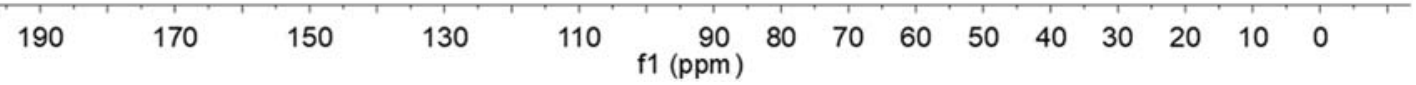


${ }^{1} \mathrm{H}$ NMR of 4 (400 MHz, $\left.\mathrm{CDCl}_{3}\right)$

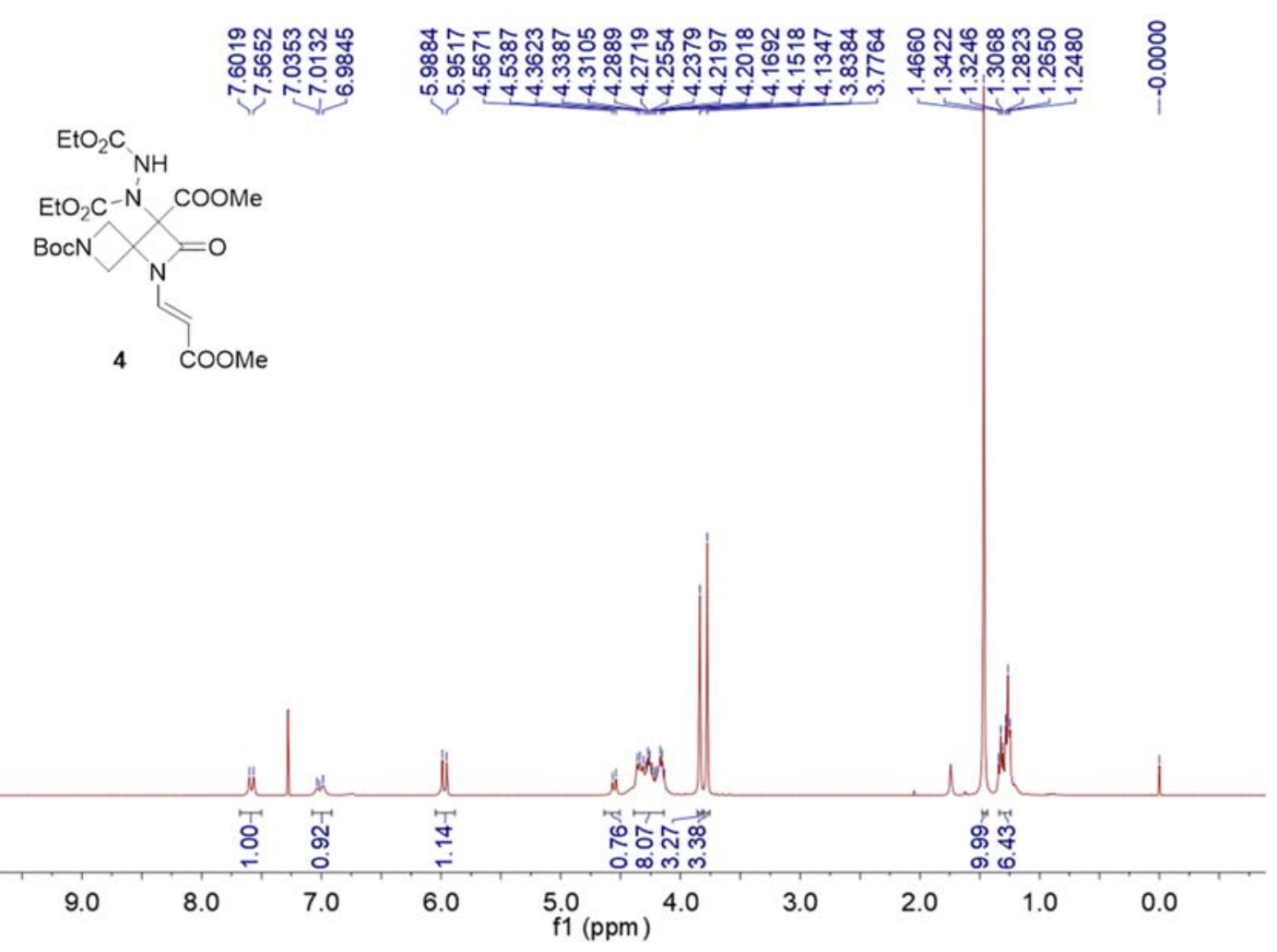

${ }^{13} \mathrm{C}$ NMR of $4\left(100 \mathrm{MHz}, \mathrm{CDCl}_{3}\right)$
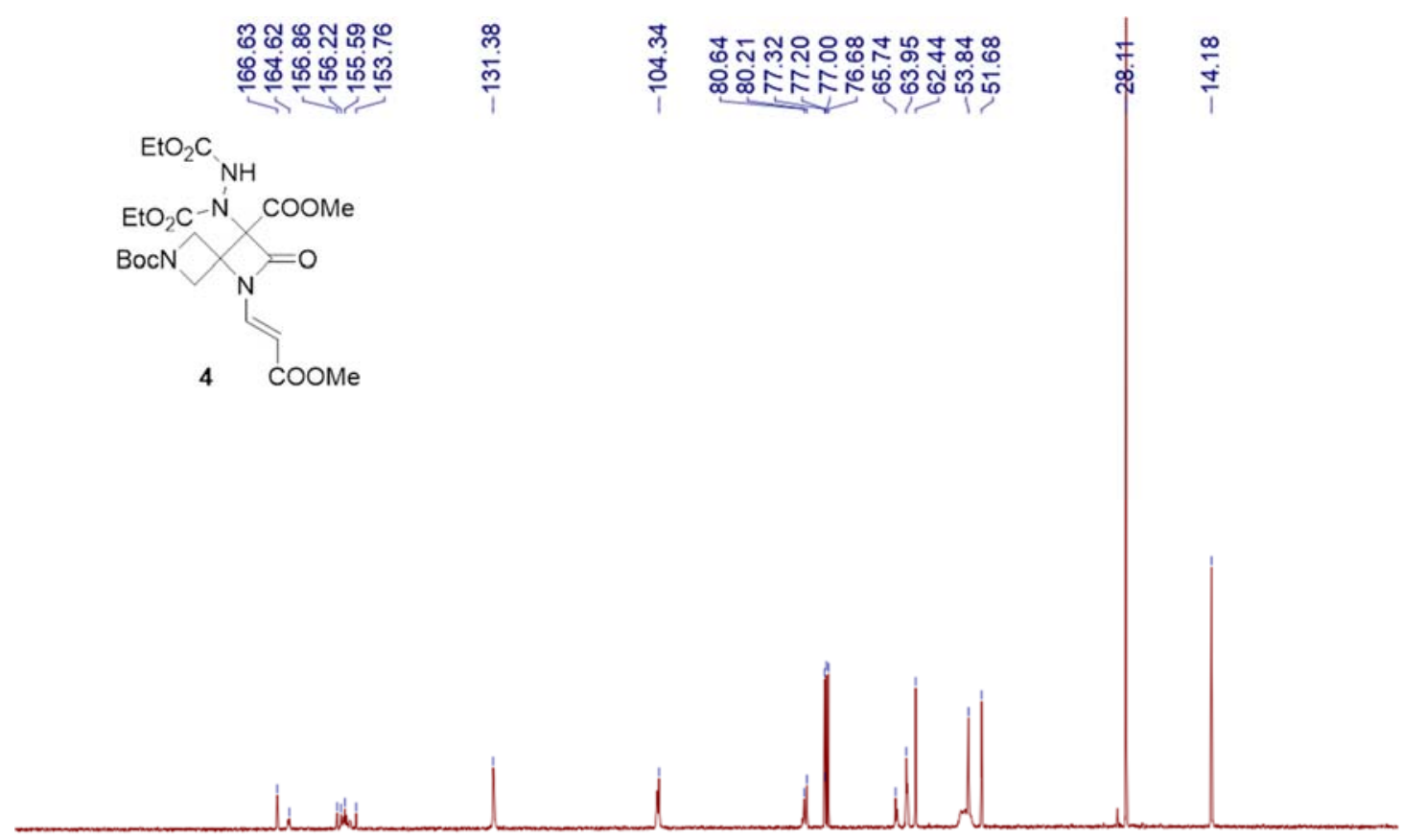

$\begin{array}{llllllllllllllll}190 & 170 & 150 & 130 & 110 & \begin{array}{r}90 \\ \mathrm{f} 1(\mathrm{ppm})\end{array} & 80 & 70 & 60 & 50 & 40 & 30 & 20 & 10 & 0\end{array}$ 
${ }^{1} \mathrm{H}$ NMR of $5\left(400 \mathrm{MHz}, \mathrm{CDCl}_{3}\right)$

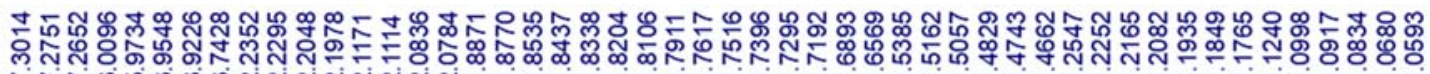

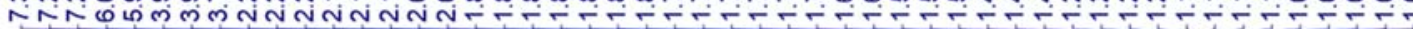
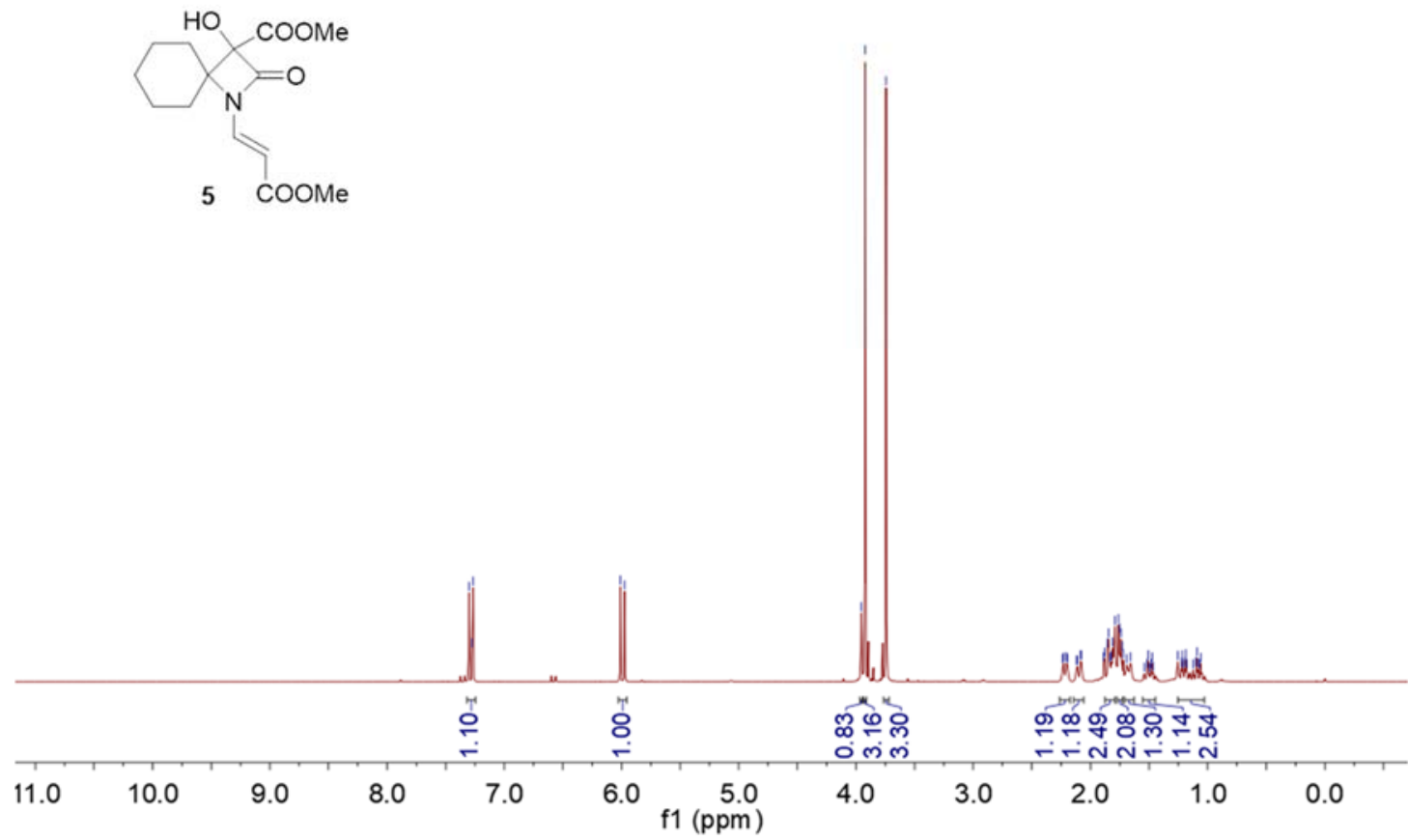

${ }^{13} \mathrm{C} \mathrm{NMR}$ of $5\left(100 \mathrm{MHz}, \mathrm{CDCl}_{3}\right)$
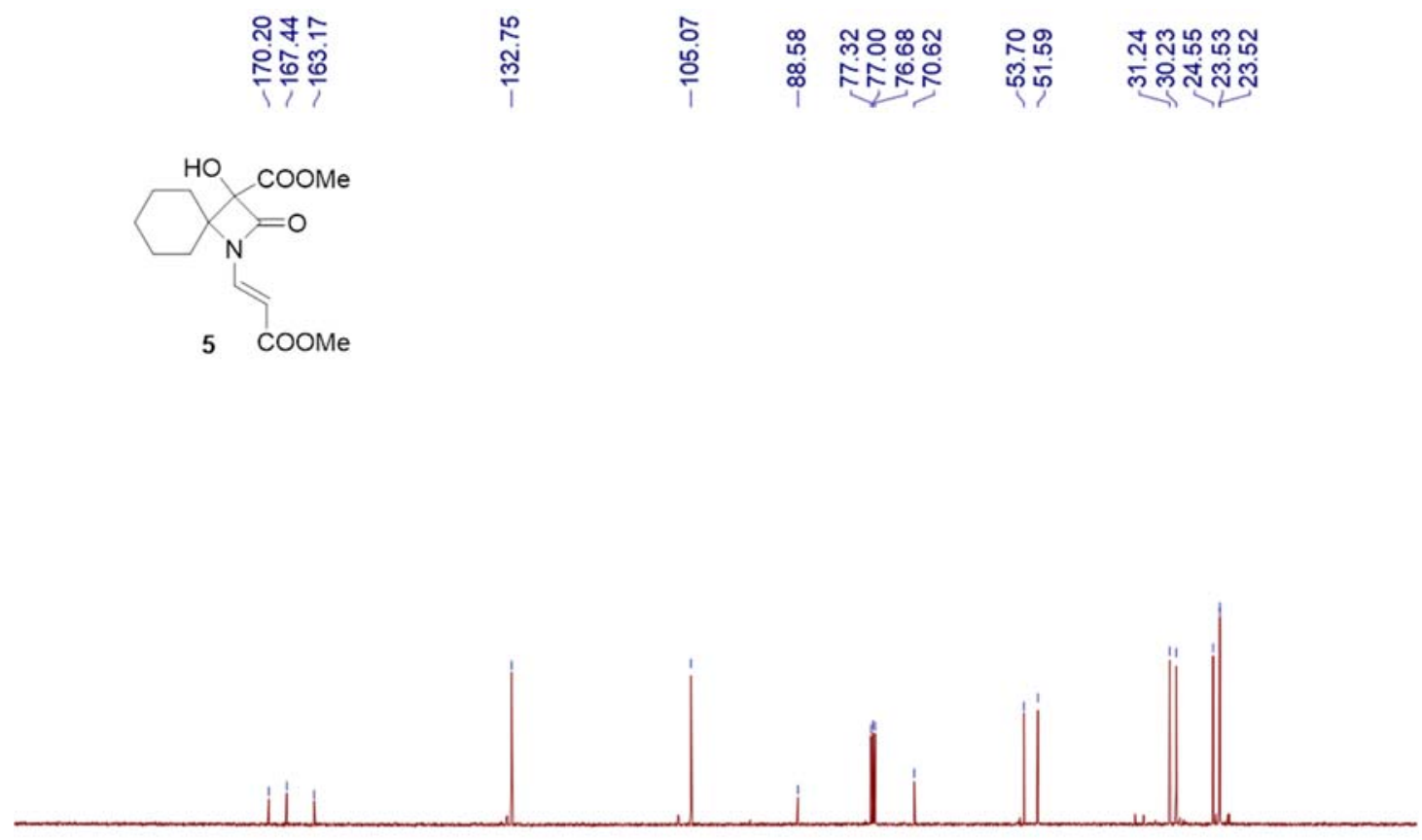

190

170

150

130

110 f1 (ppm) 90

$\begin{array}{lllllllll}80 & 70 & 60 & 50 & 40 & 30 & 20 & 10 & 0\end{array}$ 
${ }^{1} \mathrm{H}$ NMR of 6 (400 MHz, DMSO- $d_{6}$ )

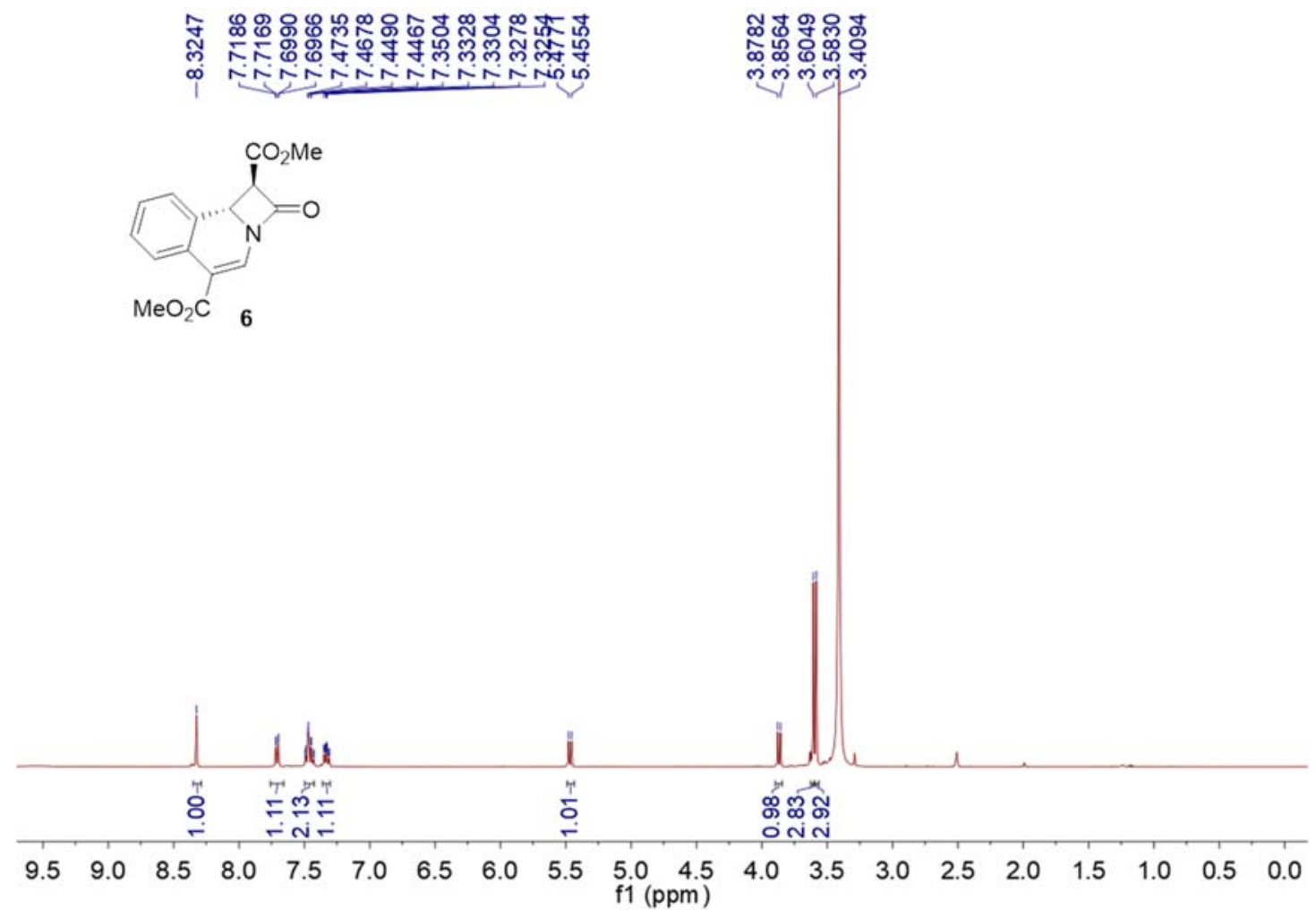

${ }^{13} \mathrm{C}$ NMR of $6\left(100 \mathrm{MHz}\right.$, DMSO- $\left.d_{6}\right)$

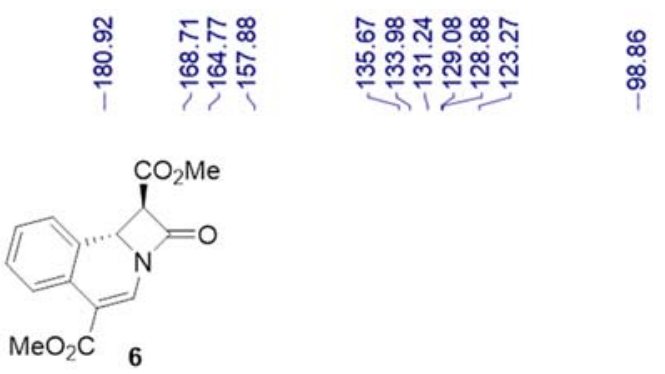

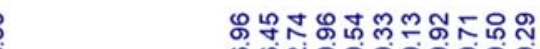

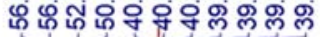

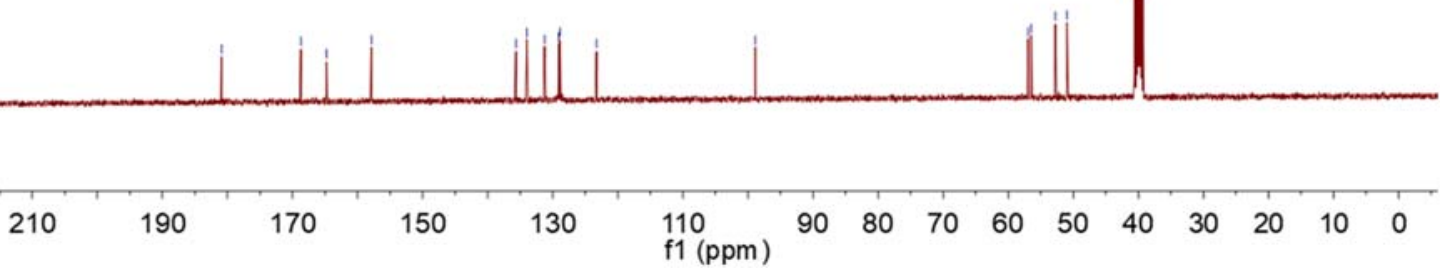


${ }^{1} \mathrm{H}$ NMR of 7 (400 MHz, $\left.\mathrm{CDCl}_{3}\right)$

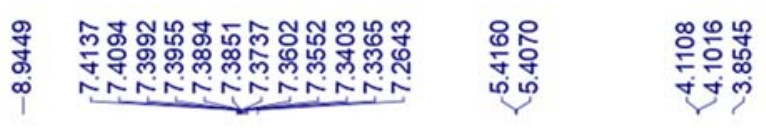

웅<smiles>COC(=O)C(C(C)=O)C(NC=O)c1ccccc1</smiles>

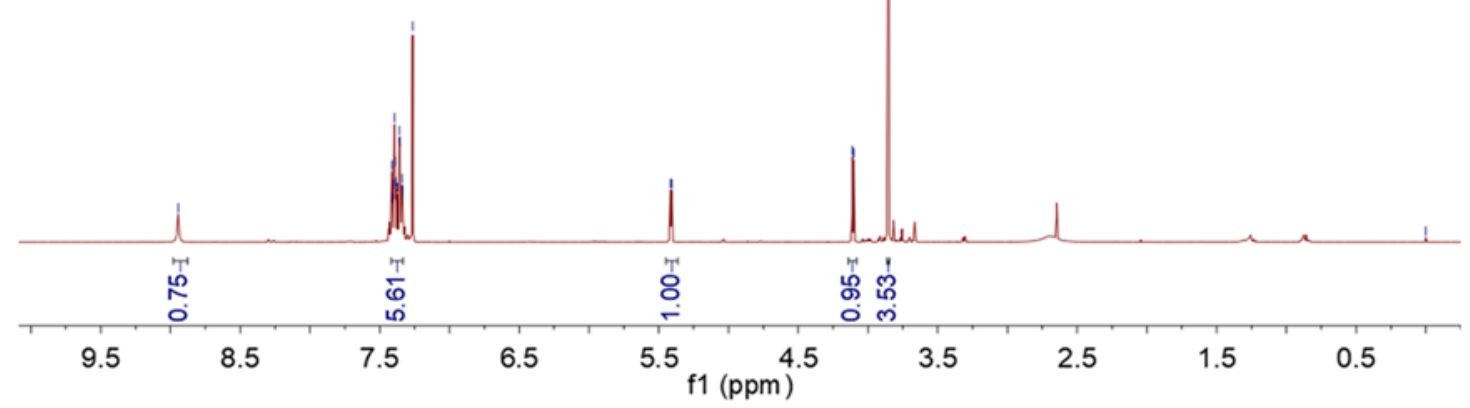

${ }^{13} \mathrm{C}$ NMR of $7\left(100 \mathrm{MHz}, \mathrm{CDCl}_{3}\right)$

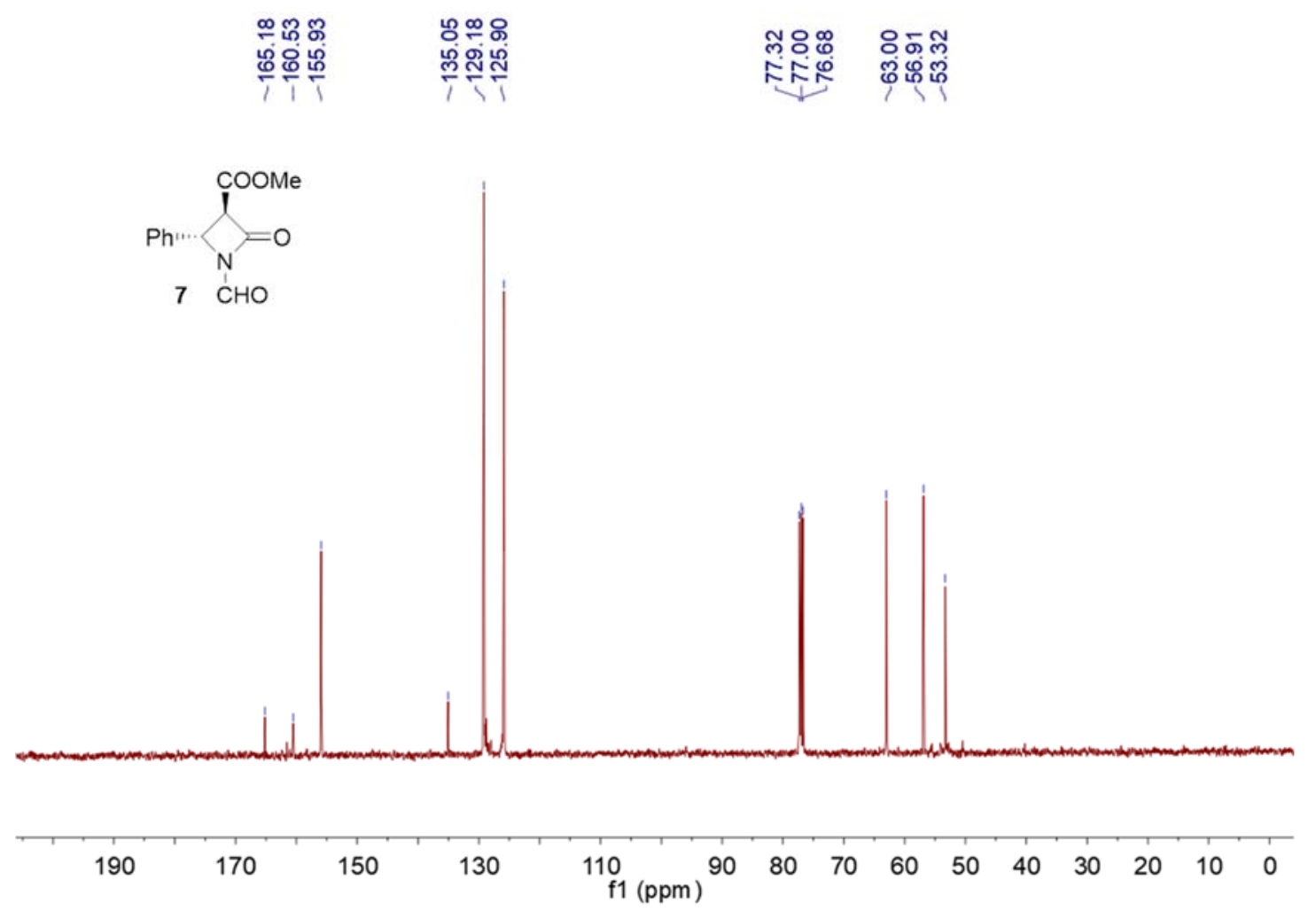

